\title{
Stepping stones to improve cardio-oncological care
}

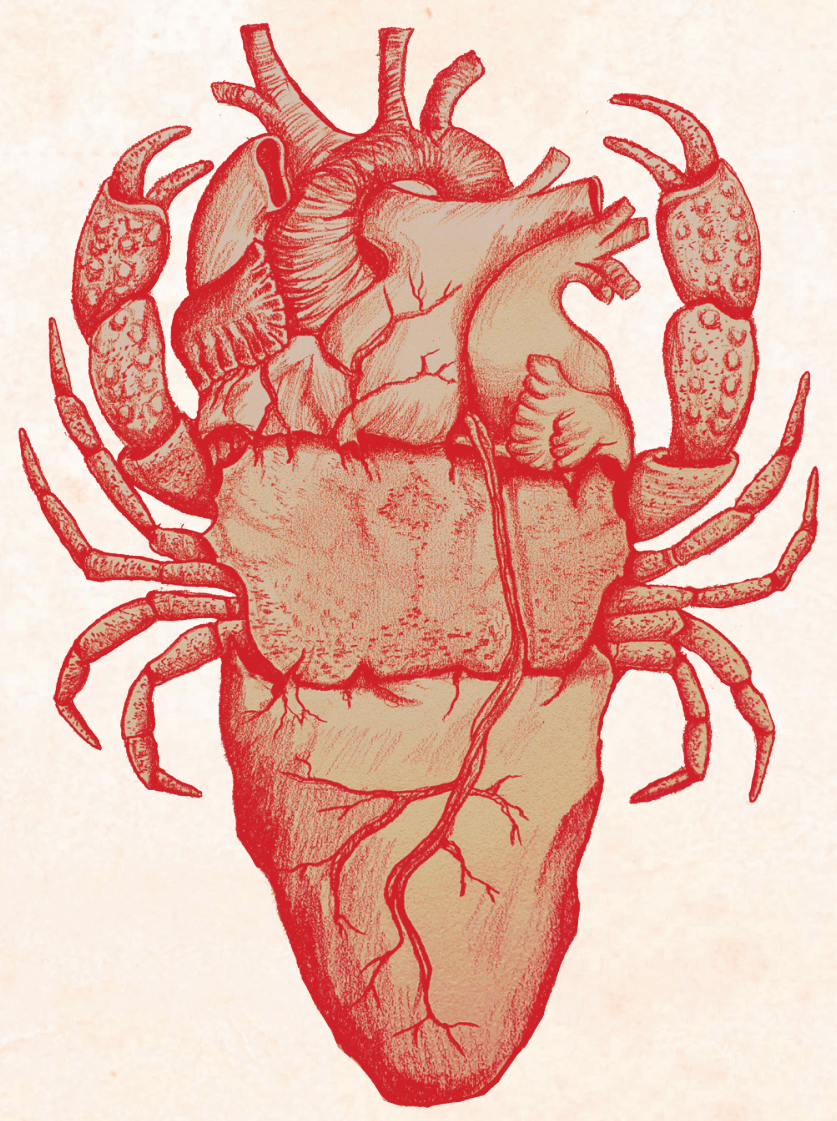

Janine Kamphuis 


\section{Stepping stones to improve cardio-oncological care}

Janine Anne Maria Kamphuis 


\section{Stepping stones to improve cardio-oncological care}

Author:

Cover illustration:

Printing:

ISBN:
Janine Anne Maria Kamphuis

Michelle Kamphuis | Tats by Mi

Gildeprint B.V.| www.gildeprint.nl

978-90-393-7339-2

All rights reserved. No part of this thesis may be reproduced, stored in a retrieval system, or transmitted, in any form or by any means without the prior permission of the author.

Financial support by the Dutch Heart Foundation for the publication of this thesis is gratefully acknowledged. 


\title{
Stepping stones to improve cardio-oncological care
}

\author{
Fundamenten voor het verbeteren van \\ cardio-oncologische zorg \\ (met een samenvatting in het Nederlands)
}

\section{Proefschrift}

ter verkrijging van de graad van doctor aan de Universiteit Utrecht op gezag van de

rector magnificus, prof.dr. H.R.B.M. Kummeling, ingevolge het besluit van het college voor promoties in het openbaar te verdedigen op

donderdag 12 november 2020 des ochtends te 9.15 uur

door

Janine Anne Maria Kamphuis

geboren op 3 augustus 1990

te Oldenzaal 
Promotoren:

Copromotoren:
Prof. dr. P.A.F.M. Doevendans

Prof. dr. F.W. Asselbergs

Dr. M.J.M. Cramer

Dr. A.J. Teske 




\section{Table of Contents}

Chapter I General introduction and thesis outline

Chapter 2 Cardio-oncology - An overview on outpatient management and I8 future developments

Chapter 3 Cancer therapy-related cardiac dysfunction of non-anthracycline 44 chemotherapeutics - What's the evidence?

Chapter 4 Cardiovascular adverse events in patients with non-Hodgkin lymphoma treated with first-line cyclophosphamide, doxorubicin, vincristine, and prednisone (CHOP) or CHOP with rituximab (R-CHOP) - A systematic review and meta-analysis

Chapter 5 Early- and late diagnosed anthracycline-induced cardiac dysfunction: echocardiographic characterization and response to heart failure therapy

Chapter 6 Cardio-oncology in a real-world setting - The clinical yield of II2 specialized cardiac care for cancer patients

Chapter 7 ONCOR - Design of the Dutch Cardio-Oncology Registry

Chapter 8 Summary and Future perspectives

Appendices Nederlandse Samenvatting

Dankwoord

Curriculum Vitae 


\section{Chapter I}

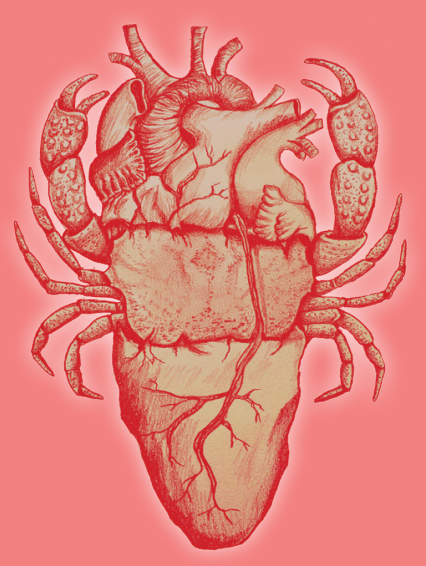


General introduction and thesis outline 


\section{Introduction}

The treatment of cancer has improved tremendously in the last decades. Next to surgery and radiotherapy, systemic treatment is one of the standard cancer treatment methods. The first chemotherapeutic agent was discovered in the I940's, when mustard nitrogen turned out to be an effective antineoplastic treatment for lymphomas [I]. During subsequent years, thousands of substances were tested for anticancer activity [2]. Several of the standard chemotherapeutic agents that are still used in clinical practice today were discovered back then. In the I960's, chemotherapeutic combination therapy led to further improved treatment response [3]. The ongoing developments have resulted in a doubling of the survival rates in Dutch cancer patients, from a 5-year survival rate of $33 \%$ in the period of 1960-I970, up to 66\% in the last decade (Figure I) [4]. At the same time, the growing aging population, together with enhanced cancer detection methods resulted in a growth of the patient population diagnosed with cancer. The downside of these developments is the growing number of patients who are at risk for adverse events of cancer treatment, such as cardiovascular toxicity.

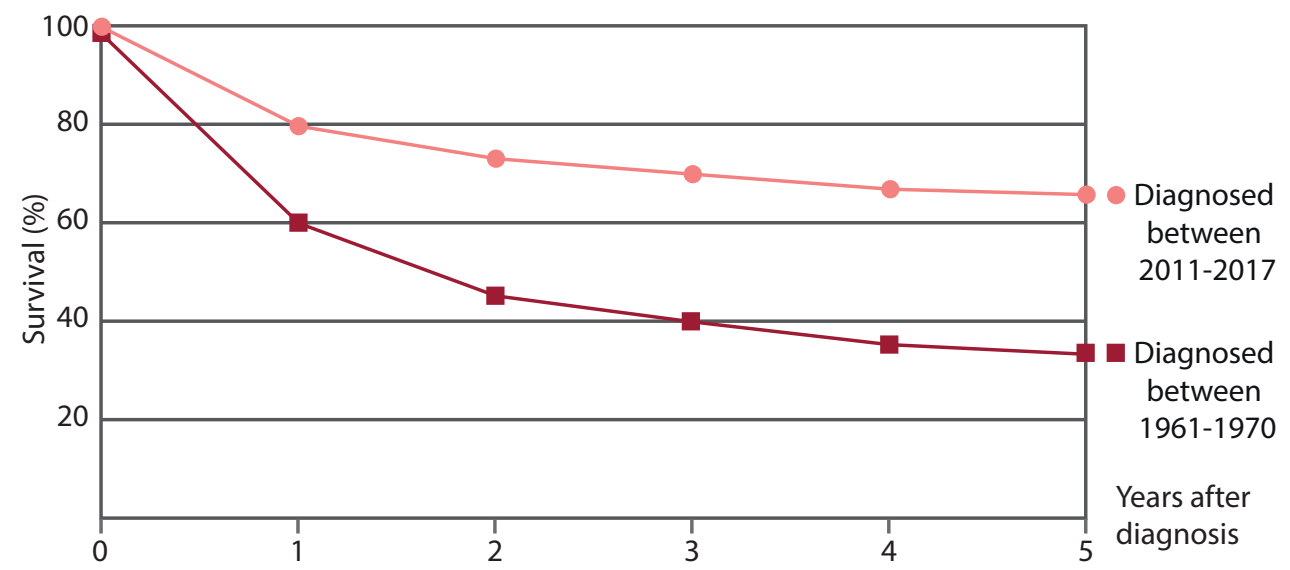

Figure I Survival of cancer patients in the Netherlands. Numbers derived from [4].

\section{Cardiotoxicity of systemic anticancer treatment}

Currently, a large number of agents are available for cancer treatment, and the number continues to rise due to ongoing developments in anticancer therapies. Many of these anticancer agents have been associated with various cardiovascular toxicities including arrhytmias, coronary artery disease, thrombo-embolic events, pericardial disease, pulmonary hypertension and myocardial dysfunction (Figure 2). Cancer therapy-related cardiac dysfunction is considered to be one of the most dangerous side effects, since it can lead to severe, refractory heart failure. The risk of cancer therapy-related cardiac dysfunction (CTRCD) is predominantly determined by the administered agent, of which anthracyclines and trastuzumab are considered to be the most cardiotoxic. 
BTK inhibitors (ibrutinib), BCR-ABL1 inhibitors (dasatinib, nilotinib, ponatinib), paclitaxel, cyclophosphamide

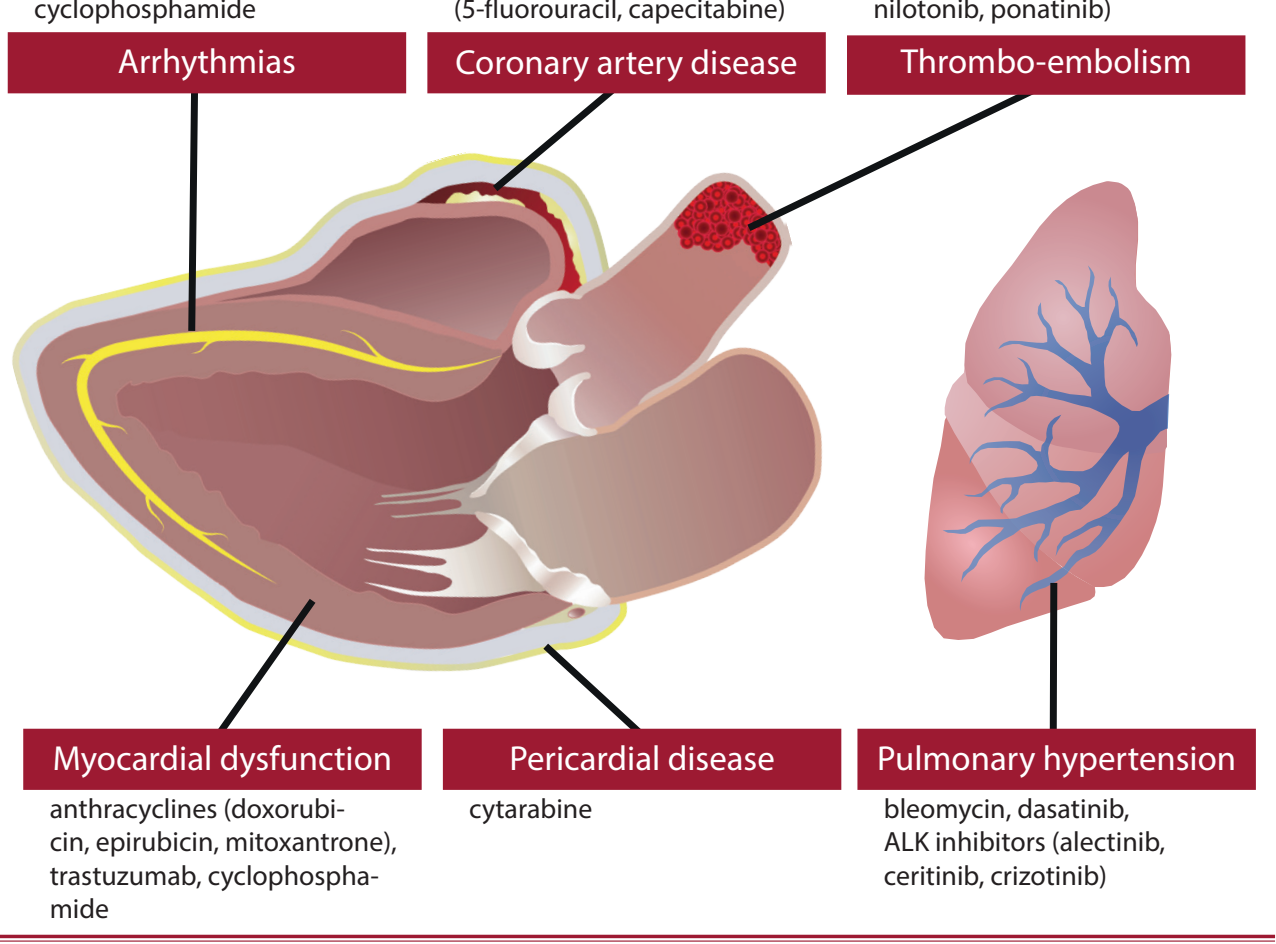

Figure 2 Overview of cardiovascular toxicities that are associated with systemic anticancer treatment. Some examples of anticancer agents associated to the specific cardiotoxicity are presented.

\section{Anthracyclines}

Anthracyclines are potent antineoplastic agents that were first introduced in the I960's [6,7], and nowadays still constitute the cornerstone in treatment regimens of, amongst others, breast cancer, sarcomas, hematological malignancies and several childhood cancers [8-13]. The antineoplastic effect of anthracyclines is mediated by the inhibition of topoisomerase II $\alpha$ in cancer cells, thereby preventing the recombination of DNA double strands, and thus DNA replication [14]. The cardiotoxic effect of anthracyclines is induced by the inhibition of topoisomerase II $\beta$ in cardiomyocytes. Topoisomerase II $\beta$ inhibition results in accumulation of double-stranded DNA breaks and mitochondrial dysfunction, leading to activation of cell death pathways and accumulation of reactive oxygen species within the cardiomyocyte [I4]. The occurrence of cardiomyocyte damage is reflected by troponin release, which can be detected during- or shortly after anthracycline infusion [15,I6]. Due to cardiac reserve and the activation of compensatory mechanisms, cardiomyocyte damage and cardiac dysfunction do not immediately have to lead to clinical symptoms [17]. This subclinical cardiac dysfunction can manifest later on, when additional cardiac stressors lead to the transition from subclinical- to clinical heart failure [I8]. 
In clinical practice, the first cases of anthracycline-induced cardiac dysfunction (AICD) were described several years after their introduction [19,20]. Subsequently, the discovery of a dose-dependent relationship led to the introduction of a maximal cumulative dose of $550 \mathrm{mg} / \mathrm{m}^{2}$, which corresponded to an incidence of $<5 \%$ congestive heart failure [2I]. Larger retrospective studies found equal incidence rates at lower cumulative doses, which led to a further restriction to $400-450 \mathrm{mg} / \mathrm{m}^{2}[22,23]$. Additional factors that are related to AICD are preexisting cardiovascular disease, anthracycline administration during childhood ( $<$ I8 years) or at older age ( $>65$ years), radiotherapy involving the heart, and concurrent treatment with other potential cardiotoxic agents such as trastuzumab [5]. However, in the absence of known risk factors, some patients can still suffer from AICD.

The optimal treatment for AICD has not been established yet. Current treatment strategies consist of the initiation of ACE-inhibitors and betablockers, following standard heart failure guidelines [5,24]. Early treatment initiation has shown beneficial effects, whereas a delayed heart failure therapy initiation is associated with poor recovery of cardiac function [25].

\section{Trastuzumab}

Trastuzumab (Herceptin $\left.{ }^{\circledR}\right)$ is a monoclonal antibody directed against the human epidermal growth factor receptor 2 (HER2). In cardiomyocytes, HER2 is involved in cardioprotective pathways that regulate apoptosis, hypertrophic growth, cell-cell contact, cell elongation, angiogenesis, and sensitivity to inotropic effects of $\beta \mathrm{I}$-adrenergic agonists [26]. Inhibition of these signaling cascades are believed to play a central role in trastuzumab-related cardiotoxicity. Trastuzumab induces cardiomyocyte dysfunction without causing structural changes as seen with anthracycline-mediated damage [27]. In general, the cardiac dysfunction is reversible after trastuzumab discontinuation, and a rechallenge of the drug can be attempted, since the cardiac dysfunction often does not reappear [27].

The overexpression of HER2, which is present in $20-25 \%$ of breast cancers, is associated with aggressive tumor growth and a high metastatic potential [28]. Breast cancer tumors with HER2 overexpression are therefore associated with a poor prognosis, compared to HER2 negative tumors. The initial phase I- and II trials with trastuzumab showed significant improvements in oncological outcomes, and high rates of cardiotoxicity were not reported [29]. Subsequently, a randomized, multicenter, phase III trial was performed, which compared trastuzumab combined with standard chemotherapy versus chemotherapy alone in metastatic breast cancer patients [30]. Cardiac dysfunction was found in $27 \%$ of patients treated concurrently with trastuzumab and anthracyclines. As a consequence, trastuzumab can only be used subsequently after anthracyclines, or concurrently with non-anthracycline chemotherapeutic agents.

In a recent meta-analysis, the proportion of severe heart failure in patients treated with trastuzumab after anthracyclines was estimated at $2.90 \%$ (95\% CI $2.16-3.72 \%$ ) [3I]. The treatment combination of trastuzumab with taxanes was associated with much lower rates of severe heart failure, with a pooled proportion of $0.92 \%(95 \% \mathrm{CI}$ 0.4I - I.64). 
Due to the beneficial effects on disease free- and overall survival, trastuzumab, often combined with chemotherapy, is currently indicated as first-line systemic therapy in early- and advanced breast cancers with HER2 overexpression. [8,32] In early breast cancer, the addition of trastuzumab to standard chemotherapy halves the recurrence and mortality risk, compared to chemotherapy alone [8].

Due to the high cardiotoxic risk, strict monitoring of cardiac function during trastuzumab treatment is mandatory according to the United States Food and Drug Administration (FDA) [33]. Left ventricular ejection fraction (LVEF) measurements should be performed prior to initiation of therapy, and every 3 months upon completion of therapy. Trastuzumab should be withheld for at least 4 weeks when the LVEF drops $\geq 16$ percentage points, or $\geq$ IO percentage points to a value below the lower limit of normal. If the LVEF returns to normal limits and the absolute decrease from baseline is $\leq \mathrm{I} 5 \%$, treatment with trastuzumab can be resumed.

\section{The establishment of cardio-oncology as a subspecialty}

The growing awareness of cancer therapy-related cardiovascular complications and the need for an interdisciplinary approach for their management has led to the establishment of the subspecialty 'cardio-oncology' in the late I990s (Figure 3)[34]. The field of cardio-oncology involves both the cardiologist as well as the (hemato-)oncologist and strives to improve cardiovascular health of cancer patients- and survivors at different stages in the disease process. Prior to oncological treatment initiation, patients with preexisting cardiovascular disease can benefit from specialized cardio-oncological care by optimizing cardiovascular status, thereby aiming to provide the most beneficial oncological treatment. Cardiac surveillance during cancer treatment focuses on early detection of cardiovascular complications and to prevent further deterioration of cardiovascular health. Screening for long-term cardiovascular effects is performed in patients at high risk, such as patients treated for hematological malignancies [35].

Whereas after its establishment, the focus of cardio-oncology was on the treatment of acute and chronic cancer therapy-induced effects, it has expanded to early detection and preventive strategies. To meet the growing need for early detection and preventive strategies, specialized cardio-oncology services have been established worldwide [36,37]. To guide health care professionals in providing cardio-oncological care, the Task Force for cancer treatments and cardiovascular toxicity of the European Society of Cardiology (ESC) has composed a position paper providing expert consensus regarding cardiovascular monitoring and decision-making before, during and after potential cardiotoxic cancer treatment [5]. Even though the position paper can assist clinicians in providing cardio-oncological care, it is not a formal clinical practice guidelines and evidence-based clinical guidelines are currently still lacking. Due to the lack of formal guidelines, cardio-oncology services have adopted different clinical approaches, according to local expertise and -needs. Outcomes and experiences of these specialized services are still scarce $[38,39]$ and the optimal strategy to provide cardio-oncological care has not been established yet. 


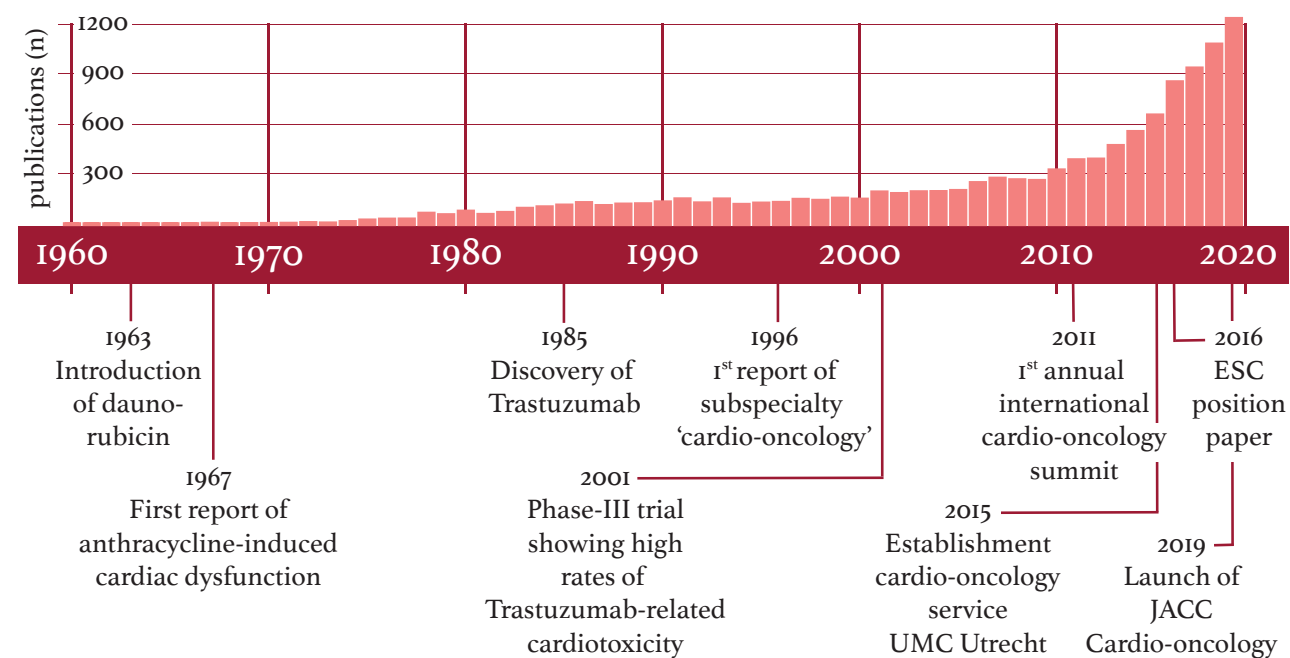

Figure 3 Overview of the evolution of 'cardio-oncology' over time. Upper panel: Number of publications per year on 'cardio-oncology' and 'cardiotoxicity'. 


\section{Aims of this thesis}

The general aim of this thesis is to describe the implementation and outcomes of a specialized cardio-oncology service, as well as to evaluate the current knowledge on cardio-oncological care. In 20I5, a specialized cardio-oncology service was established at the University Medical Center Utrecht, which provides cardio-oncological care according to a standardized healthcare pathway. Chapter 2 provides a summary of practical cardio-oncology and describes a strategy for cardio-oncology outpatient management following this standardized healthcare pathway.

One of the main activities of this cardio-oncology service is performing cardiac screening in patients at high risk for CTRCD. This risk is predominantly determined by the oncological treatment, and therefore, knowledge of cardiotoxic profiles of anticancer treatments is essential to select the patients who would benefit from cardio-oncological screening. Whereas anthracyclines and trastuzumab are well-known for their highly cardiotoxic features, several other anticancer agents are also stated to be highly cardiotoxic. In Chapter 3, the cardiotoxic profiles of five non-anthracyclines chemotherapeutic agents are evaluated. Since chemotherapeutic agents are rarely given as monotherapy, we decided that knowledge of the cardiotoxic risk profile of chemotherapeutic regimens as a whole could be relevant for clinical practice. Among the wide variety of chemotherapeutic regimens, R-CHOP is widely used in the treatment of non-Hodgkin lymphoma and contains the two potential cardiotoxic agents doxorubicin and cyclophosphamide. To evaluate the cardiotoxicity of the R-CHOP regimen, we performed a systematic review and meta-analysis, which is presented in Chapter 4.

Anthracyclines are well-known for their risk of inducing cardiac dysfunction. Therefore, screening for AICD is one of the main activities of the cardio-oncology service. Historically, AICD has been regarded as a late complication after anthracycline treatment, that clinically manifested as a form of severe heart failure, often refractory to heart failure therapy. In Chapter 5, we provide an analysis of patients who were diagnosed with AICD at the cardio-oncology service. The echocardiographic phenotype of AICD is described, as well as the response of AICD to heart failure medication, both in relation to the time between oncological treatment and AICD diagnosis.

In Chapter 6, the experiences of 4 years of specialized cardio-oncological care at the University Medical Center Utrecht according to the care pathway delineated in Chapter 2, are discussed. Our initiative to establish the national cardio-oncology registry ONCOR to collect real-world data of cardio-oncology care provided in Dutch hospitals, according to the standardized healthcare pathway is presented in Chapter 7 . The rationale and design of this registry, as well as preliminary results are presented. Finally, Chapter $\mathbf{8}$ presents the main findings of this thesis and will discuss future perspectives for the field of cardio-oncology. 


\section{References}

I Einhorn J. Nitrogen mustard: the origin of chemotherapy for cancer. Int J Radiat Oncol Biol Phys. I985;II (7):1375-8

2 Davis W, Larionov LF, Progress in chemotherapy of cancer. Bull World Health Organ. 1964;30:327-4I

3 DeVita VT Jr, Lewis BJ, Rozencweig M, Muggia FM. The chemotherapy of Hodgkin's disease: past experiences and future directions. Cancer. 1978;42(2):979-90

4 Integraal Kankercentrum Nederland, Nederlandse Kanker Registratie. Available at: https://www.iknl/ nkr-cijfers Accessed January 24, 2020.

5 Zamorano JL, Lancellotti P, Rodriguez Muñoz D, et al. 2016 ESC Position Paper on cancer treatments and cardiovascular toxicity developed under the auspices of the ESC Committee for Practice Guidelines: The Task Force for cancer treatments and cardiovascular toxicity of the European Society of Cardiology (ESC). Eur Heart J. 20I6;37(36):2768-280I.

6 Di Marco A, Gaetani M, Dorigotti Let al. (1963) Studi sperimentali sull 'attivita' antineoplastica del nuovo antibiotic daunomicina. Tumori 49:203-217

7 Dubost M, Ganter P, Maral R et al. (1964) Rubidomycin: a new antibiotic with cytostatic properties. Cancer Chemother Rep 4l: 35-36

8 Cardoso F, Kyriakides S, Ohno S, et al. Early breast cancer: ESMO Clinical Practice Guidelines for diagnosis, treatment, and follow-up. Ann Oncol. 2019;30(I0):1674

9 Casali PG, Abecassis N, Aro HT, et al. Soft tissue and visceral sarcomas: ESMO-EURACAN Clinical Practice Guidelines for diagnosis, treatment and follow-up. Ann Oncol. 2018;29(Suppl 4):iv268-iv269

Io Hoelzer D, Bassan R, Dombret H, et al. Acute lymphoblastic leukaemia in adult patients: ESMO clinical practice guidelines for diagnosis, treatment and follow-up. Ann Oncol. 2016;27(suppl 5):v69-v82

II Fey MF, Buske C; ESMO Guidelines Working group. Acute myeloblastic leukaemias in adult patients: ESMO clinical practice guidelines for diagnosis, treatment and follow-up. Ann Oncol. 2013;24 (suppl 6):vir38-43

I2 Tilly H, Gomes da Silva M, Vitolo U, et al. Diffuse large B-cell lymphoma (DLBCL): ESMO clinical practice guidelines for diagnosis, treatment and follow-up. Ann Oncol. 2015;26 (suppl 5):vir6-25

I3 Armenian S, Bhatia S. Predicting and preventing anthracycline-related cardiotoxicity. Am Soc Clin Oncol Educ Book. 2018;38:3-12

I4 Zhang S, Liu X, Bawa-Khalfe T, et al. Identification of the molecular basis of doxorubicin-induced cardiotoxicity. Nat Med. 20I2;I8(II):I639-42

I5 Cardinale D, Sandri MT, Colombo A, et al. Prognostic value of troponin I in cardiac risk stratification of cancer patients undergoing high-dose chemotherapy. Circulation. 2004;I09(22):2749-54.

I6 Auner HW, Tinchon C, Linkesch W, Tiran A, Quehenberger F, Link H, Sill H. Prolonged monitoring of troponin $\mathrm{T}$ for the detection of anthracycline cardiotoxicity in adults with hematological malignancies. Ann Hematol. 2003;82(4):218-22.

I7 Mann DL, Bristow MR. Mechanisms and Models in Heart Failure. Circulation. 2005;III:2837-2849.

I8 Eschenhagen T, Force T, Ewer MS, et al. Cardiovascular side effects of cancer therapies: a position statement from the Heart Failure Association of the European Society of Cardiology. Eur J Heart Fail 2OII;I3:I-IO.

I9 Tan C, Tasaka H, Yu KP, et al. Daunocymin, an antitumor antibiotic, in the treatment of neoplastic disease. Clinical evaluation with special reference to childhood leukemia. Cancer. 1967;20:333-53.

20 Bonadonna G, Monfardini S. Cardiac toxicity of daunorubicin. Lancet. 1969;1(7599):837.

2I Lefrak EA, Pitha J, Rosenheim S, Gottlieb JA. A clinicopathologic analysis of Adriamycin cardiotoxicity. Cancer. 1973;32(2):302-I4

22 Von Hoff DD, Layard MW, Basa P, Davis HL, von Hoff AL, Rozencweig M Muggia FM. Risk factors for doxorubicin-induced congestive heart failure. Ann Intern Med.1979;9I:710-717.

23 Swain SM, Whaley FS, Ewer MS. Congestive heart failure in patients treated with doxorubicin. Cancer. 2003;97:2869-79.

24 Ponikowski P, Voors AA, Anker SD, et al. 20I6 ESC Guidelines for the diagnosis and treatment of acute and chronic heart failure: The Task Force for the diagnosis and treatment of acute and chronic heart failure of the European Society of Cardiology (ESC). Developed with the special contribution of the Heart Failure Association (HFA) of the ESC. Eur J Heart Fail. 2016 Aug;18(8):89I-975. 
25 Cardinale D, Colombo A, Lamantia G, et al. Anthracycline-induced cardiomyopathy: clinical relevance and response to pharmacologic therapy. J Am Coll Cardiol.2010;55(3):213-20.

26 De Keulenaer, G. W., Doggen, K. \& Lemmens, K. The vulnerability of the heart as a pluricellular paracrine organ: lessons from unexpected triggers of heart failure in targeted ErbB2 anticancer therapy. Circ Res. 2010; I06: 35-46

27 Ewer M, Vooletich MT, Durand JB, et al. Reversibility of trastuzumab-related cardiotoxicity: new insights based on clinical course and response to medical treatment. J. Clin. Oncol. 2005; 23, 7820-7826

28 Slamon DJ, Clark GM, Wong SG, Levin WJ, Ullrich A, McGuire WL. Human breast cancer: correlation of relapse and survival with amplification of the HER-2/neu oncogene. Science. 1987;235(4785):177-82.

29 Nemeth BT, Varga ZV, Wu WJ, Pacher P. Trastuzumab cardiotoxicity: from clinical trials to experimental studies. Brit J Pharmacol. 20I7;174(2I):3727-3748

30 Slamon DJ, Leyland-Jones B, Shak S, et al. Use of chemotherapy plus a monoclonal antibody againstHER2 for metastatic breast cancer that overexpresses HER2. N Engl J Med. 200I;344:783-792.

3I Mantarro S, Rossi M, Bonifazi M, et al. Risk of severe cardiotoxicity following treatment with trastuzumab: a meta-analysis of randomized and cohort studies of 29,000 women with breast cancer. Intern Emerg Med. 20I6;II:I23-I4O

32 Cardoso F, Senkus E, Costa A, et al. 4th ESO-ESMO International Consensus Guidelines for Advanced Breast Cancer (ABC4). Ann Oncol. 2018;29(8):1634-1657

33 FDA. Herceptin (trastuzumab) prescribing information. Available at: https://www.accessdata.fda.gov/ drugsatfda_docs/label/20I7/I03792s5337lbl.pdf. Accessed January 30, 2020.

34 Cardinale D. A new frontier: cardio-oncology. Cardiologia. 1996;41:887-891.

35 Strongman H, Gadd S, Matthews A, et al. Medium and long-term risks of specific cardiovascular diseases in survivors of 20 adult cancers: a population-based cohort study using multiple linked UK electronic health records databases. Lancet. 2019;394(IO203):IO4I-IO54

36 Lancellotti P, Suter TM, López-Fernández, et al. Cardio-Oncology Services: rationale, organization, and implementation. Eur Heart J. 2019;40(22):1756-1763

37 Snipelisky D, Park JY, Lerman A, et al. How to develop a cardio-oncology clinic. Heart Fail Clin. 20I7;13(2):347-359

38 Pareek N, Cevallos J, Moliner P, et al. Activity and outcomes of a cardio-oncology service in the United Kingdom - a five-year experience. Eur J Heart Fail. 2018;20(I2):I72I-3I

39 Kappel C, Rushton M, Johnson C, et al. Clinical experience of patients referred to a multidisciplinary cardio-oncology clinic: an observational cohort study. Curr Oncol. 2019;26(3):e322-e327 


\section{Chapter 2}

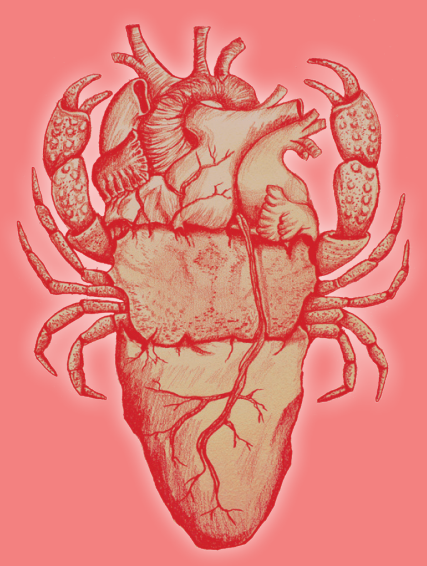




\title{
Cardio-oncology \\ An overview on outpatient management and future developments
}

\author{
Netherlands Heart Journal 20I8; 26(II):52I-32
}

AJ Teske ${ }^{\mathrm{I}}, \mathrm{M} \mathrm{Linschoten}^{\mathrm{I}}$, JAM Kamphuis ${ }^{\mathrm{I}}$, WR Naaktgeboren ${ }^{\mathrm{I}}$,

T Leiner ${ }^{2}$, E van der Wall' ${ }^{3}$, J Kuball ${ }^{4,5}$, A van Rhenen ${ }^{4}$, PA Doevendans ${ }^{1,6}$, MJ Cramer ${ }^{1}$, FW Asselbergs ${ }^{1,7,8,9}$

I Department of Cardiology, Division of Heart and Lungs, University Medical Centre Utrecht, Utrecht, The Netherlands 2 Department of Radiology, Utrecht University Medical Centre, Utrecht, The Netherlands 3 Department of Medical Oncology, University Medical Centre, Utrecht, Utrecht, The Netherlands 4 Laboratory of Translational Immunology, University Medical Centre Utrecht, Utrecht, The Netherlands 5 Department of Haematology, University Medical Centre Utrecht, Utrecht, The Netherlands 6 Netherlands Heart Institute, Utrecht, The Netherlands

7 Durrer Centre for Cardiovascular Research, Netherlands Heart Institute, Utrecht, The Netherlands 8 Institute of Cardiovascular Science, Faculty of Population Health Sciences, University College London, London, UK 9 Farr Institute of Health Informatics Research and Institute of Health Informatics, University College London, London, UK 


\begin{abstract}
Recent advances in the early detection and treatment of cancer have led to increasing numbers of cancer survivors worldwide. Nonetheless, despite major improvements in the outcome of these patients, long-term side effects of radio- and chemotherapy affect both patient survival and quality of life, independent of the oncological prognosis. Chemotherapy-related cardiac dysfunction is one of the most notorious short-term side effects of anticancer treatment, occurring in $\sim \mathrm{IO} \%$ of patients. Progression to overt heart failure (HF) carries a strikingly poor prognosis with a 2-year mortality rate of $60 \%$. Early detection of left ventricular damage by periodic monitoring and prompt initiation of HF treatment is key in improving cardiovascular prognosis. To meet the growing demand for a specialised interdisciplinary approach for the prevention and management of cardiovascular complications induced by cancer treatment, a new discipline termed cardio-oncology has evolved. However, an uniform, multidisciplinary approach is currently lacking in the Netherlands. This overview provides an introduction and comprehensive summary of this emerging discipline and offers a practical strategy for the outpatient management of this specific patient population.
\end{abstract}




\section{Introduction}

Advances in the early detection and treatment of cancer have led to increasing numbers of cancer survivors worldwide [I, 2]. Nonetheless, despite this substantial progress, long-term side effects of anticancer treatment can affect patient survival and quality of life considerably. Chemotherapy-related cardiac dysfunction (CTRCD) is one of the most notorious short-term side effects of anticancer treatment, occurring in $\sim \mathrm{IO} \%$ of patients [3]. To meet the growing demand for a specialised interdisciplinary approach for the prevention and management of cardiovascular complications, a new discipline termed cardio-oncology has emerged since the late i990s [4]. Cardiovascular toxicity due to chemo- and radiotherapy manifests itself in many other forms beyond myocardial dysfunction including, for example, hypertension, arrhythmias and valvular and coronary artery disease; these forms of toxicity fall outside the scope of this review [5]. However, the main focus of this overview is on the direct cardiotoxic effects of chemotherapy on cardiomyocyte survival. In this article, we aim to provide the clinical cardiologist, haematologists, and oncologists with an overview of this emerging discipline and share our current knowledge regarding the practical implementation of risk stratification, screening, and treatment of CTRCD. Suggested further reading on the following topics, as well as those that fall outside the scope of this overview, are summarised in Table $\mathbf{I}$.

\section{Scope of the problem}

The incidence of CTRCD is determined by multiple factors, of which the most important involve the administered chemotherapeutic agent(s) and, in the case of anthracyclines, the cumulative dose. Additionally, specific patient characteristics have been shown to be associated with a higher risk of CTRCD. Anthracyclines and trastuzumab (Herceptin ${ }^{\circledR}$ ) are among the most widely prescribed agents associated with serious cardiotoxicity.

Anthracyclines (e.g. doxorubicin) are a cornerstone in the treatment of numerous haematological and solid malignancies. In a large meta-analysis pooling data from I8 studies involving a total of almost 50,000 patients undergoing treatment with anthracyclines, the incidence of clinically overt and subclinical cardiotoxicity was reported in $6.3 \%$ (3.2-9.3\%) and $17.9 \%$ (II.6-24.2\%) of patients respectively [20]. End-stage heart failure was observed in $2-4 \%$ of patients and carries a strikingly poor prognosis, with a 2-year mortality rate of up to $60 \%$ [2I, 22]. Several attempts have been undertaken to reduce the incidence of anthracycline-induced cardiotoxicity. A dose-dependent relationship with heart failure led to restrictions in the administered cumulative dose. Other initiatives have involved the generation of numerous anthracycline analogues (e. g. epirubicin), concomitant administration of cardioprotective drugs (e. g. dexrazoxane), liposomal drug formulations, the application of prolonged infusion regimens to reduce peak plasma dose, and consecutive administration of other cardiotoxic drugs (i. e. trastuzumab), since simultaneous administration dramatically increases CTRCD incidence [I8, 23-28]. 
Nevertheless, due to fear of impaired antitumour efficacy, the implementation of several of the above-mentioned preventive actions has been limited in clinical practice. Hence, anthracycline-related cardiotoxicity still remains a significant clinical problem [29].

Trastuzumab is administered in breast cancer patients with human epidermal growth factor receptor 2 (HER2) positive tumours [30]. A meta-analysis found an overall incidence of a left ventricular ejection fraction (LVEF) decline in II.2\% of patients (RR I.83, 90\% CI I.36-2.47) [3I]. Importantly, the prognosis of trastuzumab-induced cardiotoxicity is generally more favourable when compared to anthracycline-induced cardiotoxicity, with a recovery of LVEF after timely cessation of trastuzumab administration in a majority of patients [32].

Table I Suggested further reading

\begin{tabular}{|c|c|c|c|c|}
\hline Ref\# & Author & Year & Topic & Description \\
\hline 6 & Rochette & 2015 & Pathophysiology & $\begin{array}{c}\text { Cardiotoxic mechanisms of anthracyclines and } \\
\text { trastuzumab. }\end{array}$ \\
\hline 7 & Lenneman & 2016 & Pathophysiology & $\begin{array}{l}\text { Overview of most common anticancer treat- } \\
\text { ments and their mechanism of cardiotoxicity. }\end{array}$ \\
\hline 8 & Moslehi & 2016 & $\begin{array}{l}\text { Targeted cancer } \\
\text { therapy }\end{array}$ & $\begin{array}{l}\text { Overview of cardiovascular toxicity new targeted } \\
\text { (non-anthracycline) targeted cancer therapies. }\end{array}$ \\
\hline 9 & Curigliano & 2012 & $\begin{array}{l}\text { Definitions / } \\
\text { Management }\end{array}$ & $\begin{array}{l}\text { ESMO Oncology guidelines on cardiac } \\
\text { monitoring, referral, and therapy. }\end{array}$ \\
\hline IO & Christenson & 2014 & $\begin{array}{l}\text { Early detection } \\
\text { Biomarkers }\end{array}$ & $\begin{array}{l}\text { Overview of circulating biomarkers in predicting } \\
\text { chemotherapy-induced cardiac toxicity. }\end{array}$ \\
\hline II & Thavendiranathan & $20 \mathrm{OI}$ & $\begin{array}{l}\text { Early detection } \\
\text { Echocardiography }\end{array}$ & $\begin{array}{l}\text { Echocardiographic myocardial deformation in } \\
\text { the early detection of cardiotoxicity. }\end{array}$ \\
\hline I2 & Thavendiranathan & 2013 & $\begin{array}{l}\text { Early detection } \\
\text { CMR }\end{array}$ & $\begin{array}{l}\text { The role of cardiac magnetic resonance in the } \\
\text { detection of cardiotoxicity. }\end{array}$ \\
\hline $\mathrm{I} 3$ & Plana & $20 \mathrm{OI}$ & Imaging & $\begin{array}{l}\text { EACVI Position paper on non-invasive imaging } \\
\text { modalities in cardio-oncology }\end{array}$ \\
\hline I4 & Herrmann & $20 \mathrm{OI}$ & $\begin{array}{l}\text { Risk stratification \& } \\
\text { Management }\end{array}$ & $\begin{array}{l}\text { Practical aspects regarding the cardio-oncology } \\
\text { care, including an outline of a risk assessment } \\
\text { tool. }\end{array}$ \\
\hline I5 & Zamorano & 2016 & $\begin{array}{l}\text { Risk stratification \& } \\
\text { Management }\end{array}$ & $\begin{array}{l}\text { ESC position paper on cancer treatments and } \\
\text { cardiovascular toxicity. }\end{array}$ \\
\hline I6 & Lancelotti & 2013 & Radiotherapy & $\begin{array}{l}\text { Consensus paper on imaging and management } \\
\text { of cardiovascular complications of radiotherapy }\end{array}$ \\
\hline 5 & Naaktgeboren & 2017 & Long-term outcome & $\begin{array}{l}\text { Overview on long term outcome after anticancer } \\
\text { treatment (chemo- and radiotherapy) }\end{array}$ \\
\hline I7 & van Dalen & $2 \mathrm{OII}$ & Prevention & $\begin{array}{l}\text { Cochrane review on cardioprotective interventi- } \\
\text { ons for cancer patients receiving anthracyclines }\end{array}$ \\
\hline I8 & Kalam & 2013 & Prevention & $\begin{array}{l}\text { Systematic review on cardioprotective therapy } \\
\text { for prevention of cardiotoxicity with } \\
\text { chemotherapy }\end{array}$ \\
\hline I9 & Johnson & 2017 & Training & $\begin{array}{l}\text { Paper exploring training programs for medical } \\
\text { specialist in cardio-oncology }\end{array}$ \\
\hline
\end{tabular}




\section{Definition}

Multiple definitions of CTRCD have been proposed in the literature to date, although a consensus is currently still lacking [33]. The most widely adapted definition is a decrease in LVEF of more than to percentage points to a value below the lower limit of normal, irrespective of symptoms. The American Society of Echocardiography and the European Association of Cardiovascular Imaging (EACVI) define an LVEF of 53\% on echocardiography as the lower limit of normal [13]. Subclinical CTRCD is defined as a global longitudinal strain (GLS) with $>15 \%$ relative reduction from baseline with preservation of LVEF [13]. Unfortunately, this definition does not cover other signs of cardiotoxicity, such as the detection of cardiac troponin release.

\section{Pathophysiology}

The pathophysiological mechanisms leading to CTRCD are complex, incompletely elucidated, and differ among chemotherapeutic agents $[7,8]$. Traditionally, for agents that have a direct effect on cardiomyocytes, two types of cardiotoxicity have been proposed [24]. Type I cardiotoxicity is characterised by irreversible damage and related to the cumulative administered dose. Anthracyclines are most well-known for their association with type I cardiotoxicity. Mechanisms believed to play a role in this type of cardiotoxicity are multifactorial and involve (I) the generation of excess reactive oxygen species, (2) accumulation of toxic anthracycline metabolites that interfere with calcium handling and thereby disrupt sarcomere structure and function, (3) interaction with transcription factor topoisomerase II $\beta$, and (4) mitochondrial dysfunction [6, 34, 35]. Type II cardiac damage is believed to cause temporary, reversible dysfunction in a dose-independent manner [32]. Trastuzumab is a classical type II agent, which binds to the HER2 receptor and thereby inhibits downstream associated signalling cascades. It is conceivable that the inhibition of these pathways plays a central role in trastuzumab-associated cardiotoxicity, but the exact mechanism still remains to be discovered [36]. Although the subdivision into type I and type II cardiotoxicity is arbitrary, as persistent LV dysfunction has also been observed in patients treated solely with type II agents, this subdivision is nevertheless still widely applied [37].

\section{Risk stratification}

Cardiovascular management of patients receiving cardiotoxic treatment is based on the timely recognition of those at high risk of developing CTRCD. Accurate risk stratification is crucial to enable an effective pre-selection of patients that should be referred to a cardio-oncological team prior to, or in an early phase of, anticancer treatment.

\section{Treatment-related risk factors}

Not all chemotherapeutic agents are (equally) cardiotoxic, as shown in Supplementary Table $I$. In this table, we subdivided a subset of the most commonly used chemotherapeutic 
agents into four categories, based on the incidence of LV dysfunction reported in the literature (group I: $<\mathrm{I} \%$, group 2: I-5\%, group 3: 5-IO \% and group 4: $>\mathrm{IO} \%$ ). It should be noted that the applied definition of LV dysfunction varies between the studies, and that the incidence is an approximation of risk. One of the most important risk factors for CTRCD in agents that cause type I cardiotoxicity is the administered dose. In patients that require more intensive regimens or have a history of previous malignancy for which they were treated with type I agents, it is important to take the cumulative dose into consideration. However, some patients develop CTRCD even with doses far below the maximum cumulative dose [23]. Therefore, a tolerated and 'safe' dose seems to be highly dependent on the presence of patient-related risk factors.

\section{Patient-related risk factors}

Patient-related risk factors that have been identified thus far include female gender, black race, exposure to cardiotoxic drugs at a young or old age $(<15$ and $>65$ years), previous or concomitant chest radiation therapy, obesity and classical cardiovascular risk factors including hypertension and diabetes mellitus [ $15,20,38]$. Having $\geq 3$ of these risk factors has been associated with a 5-6 times higher risk of cardiotoxic side effects compared to patients without any risk factors [20]. Nonetheless, in the absence of all these known determinants, some patients still develop severe CTRCD, indicating that unknown factors contribute to individual susceptibility. It is conceivable that the individual genetic profile plays a considerable role in modulating individual risk [39].

\section{Risk prediction models}

A few risk prediction models have been published in the literature thus far [I4, 40, 4I]. The Cardiotoxicity Risk Score (CRS) proposed by the Mayo Clinic takes both patient and treatment risk factors into account (Table 2; [I4]). This model addresses the a priori risk

Table 2 Cardiotoxicity Risk Score (CRS)

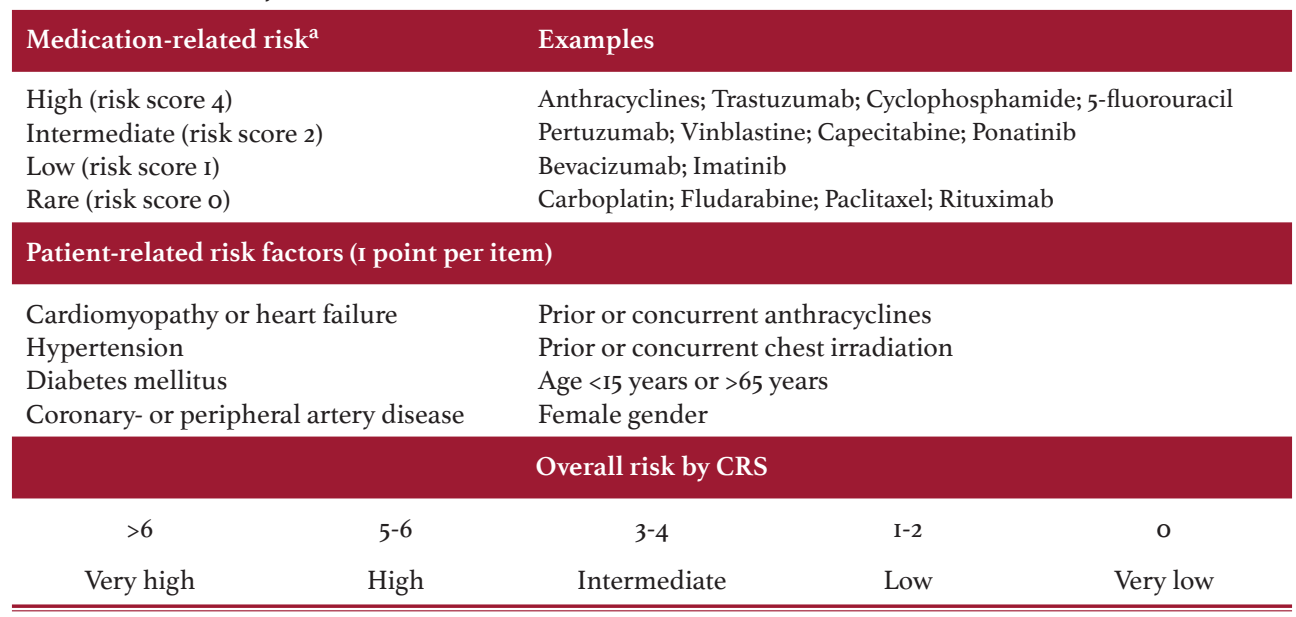

\footnotetext{
${ }^{\text {a }}$ The highest medication-related risk score (e.g. 4, 2, I or o) is used for calculation of the CRS. Adapted from [I4].
} 
of developing CTRCD. Patients with a CRS score of $\geq 4$ could benefit from cardiological consultation during and after chemotherapeutic treatment, and those with high risk scores should be closely monitored during and after treatment. However, this risk model, as well as the other models, has not been validated in a prospective setting and its real clinical value remains to be determined. In the absence of validation, the European Society of Cardiology (ESC) therefore currently does not advise the use of a particular model in the position paper on cancer treatments and cardiovascular toxicity released in 2016 [15]. Instead, the committee stresses the importance of clinical judgement in individual risk assessment, which includes clinical history, physical examination and evaluation of cardiac function pre-chemotherapy.

\section{Early detection of myocardial damage}

\section{Circulating biomarkers}

Due to the minimal invasiveness, limited costs, and low inter-observer variability, biomarkers constitute an appealing approach to aid in the early detection of subclinical cardiotoxicity (Figure I). Most studies have assessed the potential of classical cardiac biomarkers, i. e. cardiac troponin (cTn) and N-terminal pro-B-type natriuretric peptide (NT-proBNP) [Io]. Troponins seem to be the most promising candidates, both in patients treated with anthracyclines and various agents used for targeted-therapy (e. g. trastuzumab) [Io, 42]. Nevertheless, repeated sampling is currently necessary to detect cTn elevations, as the optimal timing to reach maximal sensitivity has not yet been established [42].

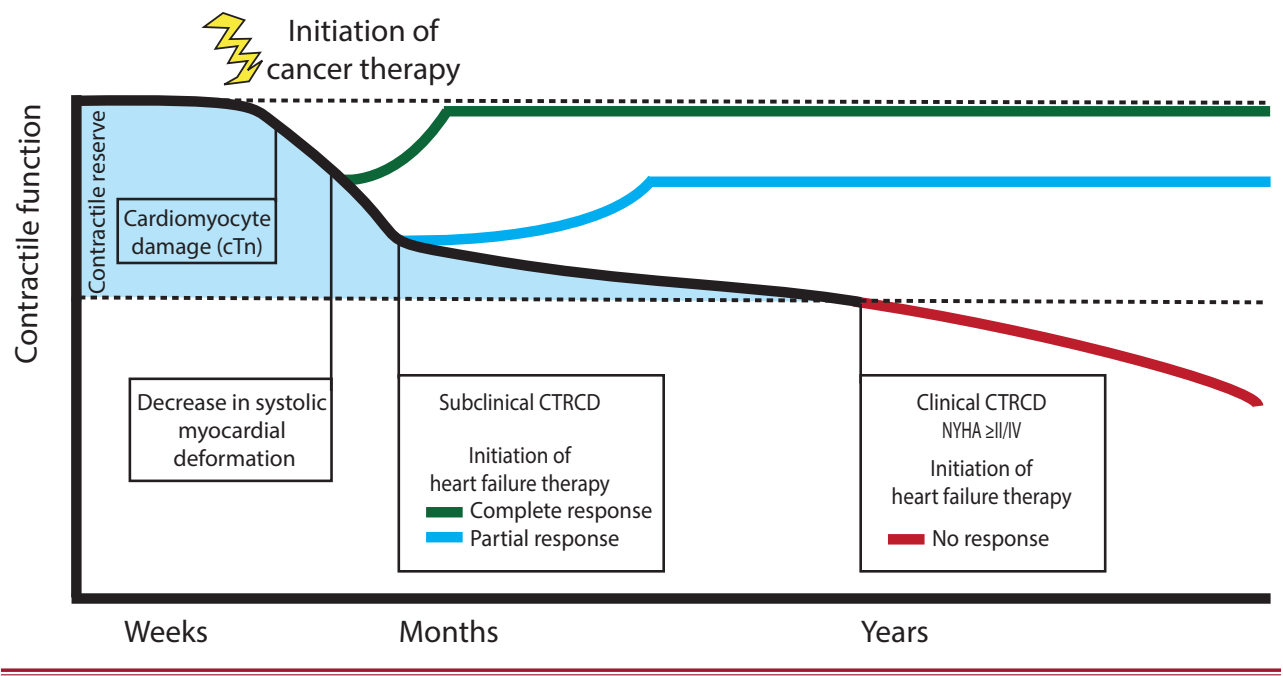

Figure I Time frame of detection and treatment of cardiotoxicity. Early initiation of heart failure treatment (green, blue line) leads to better outcomes regarding recovery of contractile function.Initiation of heart failure treatment at time when symptoms are present (red line) results in poor outcomes regarding recovery of cardiac function. cTn Cardiac troponin, CTRCD chemotherapy-related cardiac dysfunction, NYHA New York Heart Association classification. 
Multiple-gated acquisition scan

Since the I970s the mainstay imaging modality for the screening and monitoring of cardiac function in oncology patients has been the multiple-gated acquisition scan [43]. Unfortunately, the only measurement that can be derived from these scans is the LVEF, which is less sensitive for early detection of CTRCD. Another important concern is the radiation exposure ( $\sim$-IO mSv/scan) in patients undergoing serial assessments. For example, the Dutch Guidelines for Breast Cancer recommend that patients receiving trastuzumab therapy should undergo cardiac evaluation to determine the LVEF before the start of treatment and subsequently once every 3 months during treatment [44]. The cumulative radiation exposure in patients treated with this agent for I year thereby equals $\sim 25-50 \mathrm{mSv}$, which is comparable to $250-500$ chest radiographs or 4-8 CT angiography procedures.

\section{(Strain) echocardiography}

Echocardiography is the most suitable imaging modality for the evaluation of patients in preparation for, during, and after cancer therapy, because of its wide availability, easy repeatability, versatility, lack of radiation exposure, and safety in patients with concomitant renal disease. Furthermore, echocardiography allows a comprehensive evaluation of most cardiac structures and multiple parameters besides the LVEF. It has been recommended as the first line screening tool to assess cardiac function in this specific patient population [I3]. In particular, the measurement of LVEF by $3 \mathrm{D}$ echocardiography has been shown to be feasible and accurate with an error of $<5 \%$ (compared to a IO\% variation in biplane LVEF calculation) [45]. Echocardiographically derived GLS calculates the systolic deformation of the myocardium by a commercially available speckle tracking algorithm (Figure 2; [46]). This parameter reflects contractile function and is well validated in healthy subjects and in a variety of myocardial disease states. The GLS has been shown to be the single best parameter to predict CTRCD, as a decrease of this parameter is often seen before a relevant reduction of LVEF is observed [II].

\section{Cardiac magnetic resonance}

Cardiac magnetic resonance (CMR) is considered the reference standard for the assessment of ventricular function [47]. Due to its superiority in myocardial tissue characterisation, CMR is suitable to detect early tissue damage following chemotherapy. Early changes in tissue composition include myocardial edema with an increase in LV mass, inflammation, and decrease in myocardial strain [I2]. Within months after initiation of therapy LV end-systolic volume increases, and with Ti mapping techniques diffuse interstitial fibrosis, a hallmark of anthracycline-induced cardiotoxicity, can be detected and quantified (Figure 3a-c). The absence of focal fibrotic lesions results in a lack of late gadolinium enhancement, although contrast-enhanced CMR can be used to exclude other causes of myocardial dysfunction in these patients, such as myocardial infarction. Although unique insights can be obtained with MRI, due to costs and availability CMR is presently not suitable as the first-line imaging technique for regular follow-up imaging. 


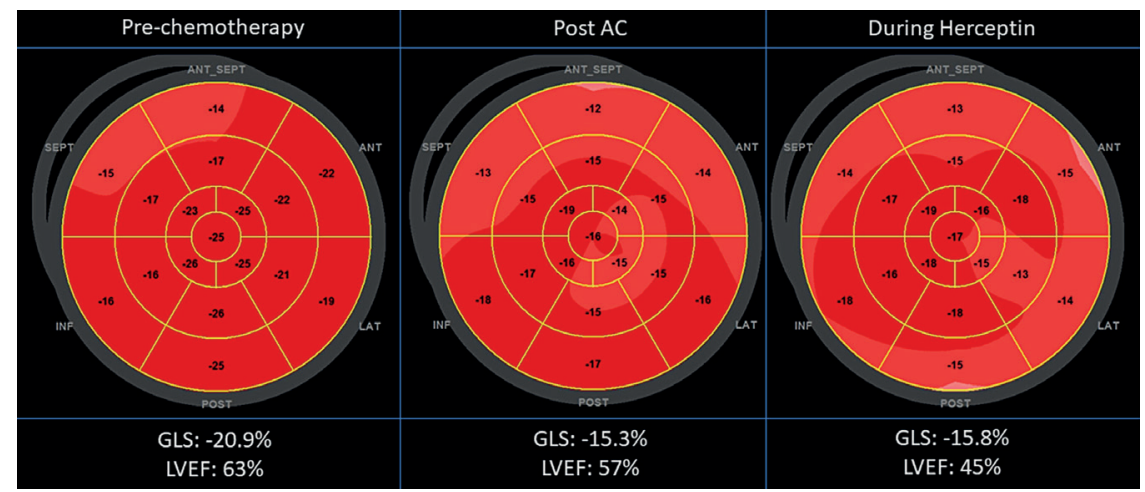

Figure 2 Echocardiographic deformation imaging. Longitudinal follow-up of a 5I-year-old female with breast cancer with a high cardiovascular risk (Cardiotoxicity Risk Score 7: female, hypertension, concurrent anthracyclines, and high-risk agent trastuzumab). After the initial $4 \times \mathrm{AC}$ (adriamycin-cyclophosphamide) there was a significant decrease of $>15 \%$ in global longitudinal strain (GLS) with preservation of left ventricular ejection fraction (LVEF). During the trastuzumab treatment there was a subsequent decrease in LVEF of $>\mathrm{IO} \%$. After interruption of the trastuzumab treatment, the LVEF showed a complete recovery

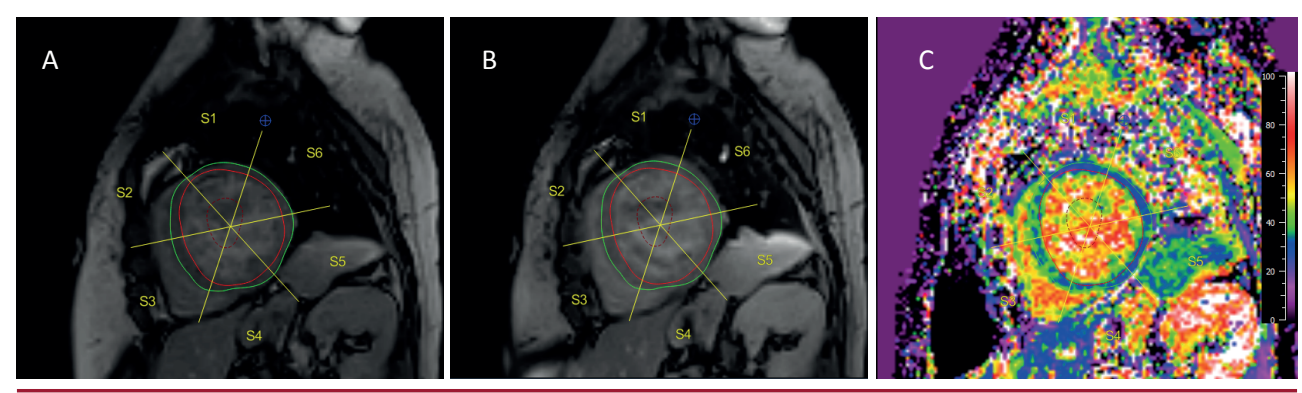

Figure 3 Cardiac magnetic resonance imaging. Cardiac magnetic resonance imaging with Ti mapping in a female breast cancer survivor, treated with anthracyclines. Extracellular volume fraction (ECV) is a non-invasive measurement of diffuse myocardial fibrosis and can be calculated from the haematocrit; pre-contrast (a), post-contrast (b) Ti maps. In this patient, the ECV map (c) reveals diffuse elevated ECV values up to $42 \%$ (normal is $<28 \%$ ), in particular in the septal segments, reflecting widespread myocardial fibrosis after anthracycline exposure

\section{Therapeutic interventions}

With the exclusion of cancer patients in all high-impact heart failure intervention randomised controlled trials regarding the efficacy of, for example, ACE inhibitors and beta-blockers, the response rate of patients with CTRCD to conventional heart failure therapy has not been thoroughly investigated and evidence-based decision-making on optimal treatment is lacking [48]. In a single-centre study by Cardinale et al. $(n=215)$, treatment response in patients with a decline of LVEF to $\leq 45 \%$ was highly dependent on the timing of treatment initiation [49]. The response rate was the highest (64\% responders) among patients with CTRCD that received heart failure treatment $<2$ months after detection of LV impairment and decreased to only 7\% after 4-6 months. Remarkably, no response was 
observed in patients with CTRCD that received treatment $\geq 6$ months after the last chemotherapeutic cycle (Figure 4a). Hence, detecting the development of CRTCD as soon as possible guides the optimal timing of treatment initiation, since this seems to be particularly crucial for treatment response. However, in a follow-up study by the same group, a large proportion of patients did not show recovery of cardiac function despite early initiation of conventional heart failure treatment. Only iı\% showed full recovery to a mean LVEF of $6 \mathrm{I} \%$. Cardiac function was partially restored in $7 \mathrm{I} \%$ of the patients, to a mean LVEF of $54 \%$. Notably, I8\% of patients did not respond to treatment, with a mean LVEF at the end of the study of $38 \%$ (Figure $4 \mathrm{~b}$; [3]). Cardiac outcome for partial and non-responders is significantly worse, including heart failure requiring hospitalisation and cardiac-related death (Figure 4c; [49]).

Currently, the only practice guidelines on the management of cardiovascular toxicity induced by anticancer treatment have been released by the European Society of Medical Oncology (ESMO) [9]. Guidelines from the ESC and the American Heart Association are still lacking, albeit the ESC recently released the first position paper on cancer treatments and cardiovascular toxicity [I5].

The evidence for the use of cardioprotective agents to counteract LVEF decline in patients treated with anthracyclines is marginal, with the exception of dexrazoxane [I7, 50]. Cardioprotective agents to prevent trastuzumab-related cardiotoxicity are largely unexplored. The results of the first randomised controlled trials investigating the cardioprotective effect of candesartan and carvedilol were recently published, showing no protection against LVEF decline [5I].

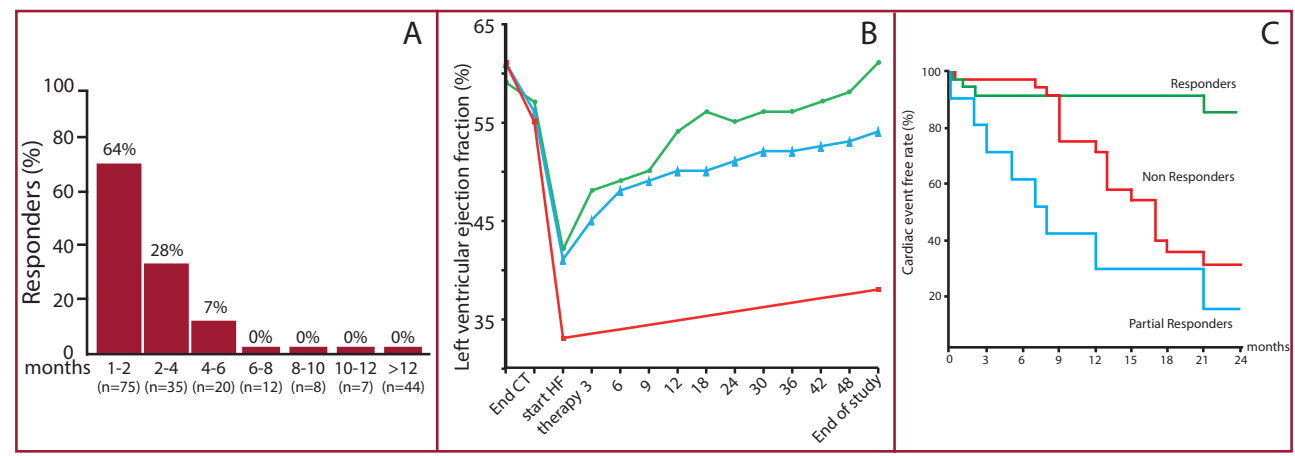

Figure 4 Response and outcome to heart failure (HF) treatment in patients with chemotherapy-related cardiac dysfunction. (a) Percentage of (partial) responders according to the time elapsed from diagnosing left ventricular dysfunction and start of HF therapy. (b) Left ventricular ejection fraction in patients with cardiotoxicity and with no (square/red), partial (triangle/blue) or full (dot/green) recovery following heart failure therapy. (c) Cumulative cardiac event rate during follow-up. Reprinted from: $[3,49]$. CT Chemotherapy 


\section{Roadmap towards outpatient management}

Collaboration between the Departments of Cardiology, Radiology, Haematology and Oncology resulted in a specialised cardio-oncology healthcare pathway, which was launched at the University Medical Centre Utrecht, the Netherlands early in 2015. The aim of this initiative is to improve cardiac outcome in oncology patients by (I) identifying patients at high risk of developing CTRCD before chemotherapeutic treatment is initiated, (2) screen and monitor high-risk patients to enable early detection of (subclinical) cardiac dysfunction which (3) facilitates early treatment initiation in order to improve overall cardiovascular outcome. Patients are monitored up to I year after the end of chemotherapy, as a majority of the patients described in the literature develop CTRCD within this time frame [3]. It should be noted that long-term follow-up data in these patients are scarce. Our in-house protocol is delineated in Figure 5.

\section{Registration procedure}

Patients are referred by the oncologist/haematologist based on in-hospital protocols on cardio-oncology referral. Main indications for referral are planned treatment with cardiotoxic agents, cardiac evaluation before (autologous or allogeneic) stem-cell transplantation (SCT), or patients presenting with complaints suggestive of underlying cardiac disease (e. g. heart failure, ischaemia, or arrhythmias). The baseline risk is determined based on the Cardiotoxicity Risk Score (CRS) (Table 2). This risk score has been slightly adjusted from its initial publication [I4] regarding the risk attributed to the different chemotherapeutic agents (see Supplementary table). Albeit this risk model has not been prospectively validated, we have chosen to incorporate it in our in-house protocol to ensure objective and uniform risk assessment and limit inter-physician variability. All patients pre-SCT as well as high-risk patients (CRS $\geq 4$ ) that will have to undergo treatment with cardiotoxic agents are seen at our cardio-oncology outpatient clinic. Childhood cancer survivors and ex-Hodgkin patients are referred to the LATER and BETER [Dutch for 'better'] outpatient clinic respectively [52].

\section{Initial cardiac evaluation}

Preferentially, the first cardiac evaluation is performed before chemotherapeutic treatment is initiated. This first assessment involves exploration of the cardiac and oncological history (including a detailed assessment of known cardiovascular risk factors as well as past and planned chemotherapeutic regimens), physical examination, laboratory analysis (including kidney function, NT-proBNP and cTn), and an ECG. Furthermore, a complete echocardiographic evaluation is performed to determine cardiac dimensions, valvular function, LVEF (preferably 3D), GLS, diastolic function, right ventricular (RV) function and RV systolic pressure. Reference values can be obtained from the current EACVI guidelines [13]. In short, an LVEF of $>53 \%$ on $2 \mathrm{D} / 3 \mathrm{D}$ echocardiography and a GLS of $-19.7 \%$ $(-20.4 \%$ to $-\mathrm{I} 8.9 \%)$ are considered normal. 


\section{Individual follow-up}

The interval of follow-up for patients who are treated with agents associated with type I cardiotoxicity is determined by the baseline CRS (Figure 5). The follow-up duration at the cardio-oncology clinic is typically up to I year after the last cycle of chemotherapy. After this period, further cardiac assessment takes place at the BETER outpatient clinic if the patient is found to be eligible [52]. Patients treated with trastuzumab are seen pre-chemotherapy, once every 3 months during treatment, and I year after the end of treatment. In asymptomatic patients that develop subclinical CTRCD, the monitoring interval is intensified as these subjects are at high risk for developing heart failure.

\section{Additional examinations}

CMR is recommended in patients with clinical CTRCD and can be considered in patients with subclinical CTRCD. Measurements include LVEF, RVEF, delayed enhancement imaging, Ti $\rho$-mapping, and determination of GLS. Ischaemia detection using adenosine stress imaging is performed if there is a history of coronary artery disease, suspected ischaemia, or $\geq 2$ cardiovascular risk factor(s) (smoking, diabetes, hypertension, hypercholesterolaemia and a positive family history) in men $>40$ years and post-menopausal women. Patients with severe CTRCD (LVEF $<45 \%$ ) or a positive family history for cardiomyopathies are offered a referral for genetic counselling as part of our local policy, as genetic variants in cardiomyopathy-associated genes may have predisposed these patients to CTRCD [53, 54].

\section{Management of patients with CTRCD}

Multidisciplinary meetings attended by the cardio-oncologist and oncologist/haematologist take place to discuss cases with (subclinical) CTRCD. At these meetings, the effect of modification(s) to the chemotherapeutic regimen to decrease cardiovascular toxicity is weighed against the consequences of these alterations for the oncological prognosis. Furthermore, in patients that have LV dysfunction prior to the initiation of cancer treatment, the optimal treatment regimen is chosen through shared decision-making.

\section{Type I cardiac dysfunction}

In patients with clinical CTRCD, treatment with ACE inhibition (preferentially enalapril) and a beta-blocker (preferentially carvedilol) is indicated. In patients with contraindications for ACE inhibition, an angiotensin-receptor blocker can be considered. Patients that show signs/symptoms of congestion receive loop diuretics. These recommendations are based on two single-centre studies $[3,49]$. The value of other agents or the optimal dose are currently unknown. At the moment, there is no evidence that treatment with other heart failure drugs (e.g. aldosterone antagonists) has any added value.

\section{Type II cardiac dysfunction}

Upon development of type II cardiac dysfunction, the causal chemotherapeutic agent(s) should be discontinued immediately. After 3 -4 weeks, the LVEF and GLS are re-assessed. 


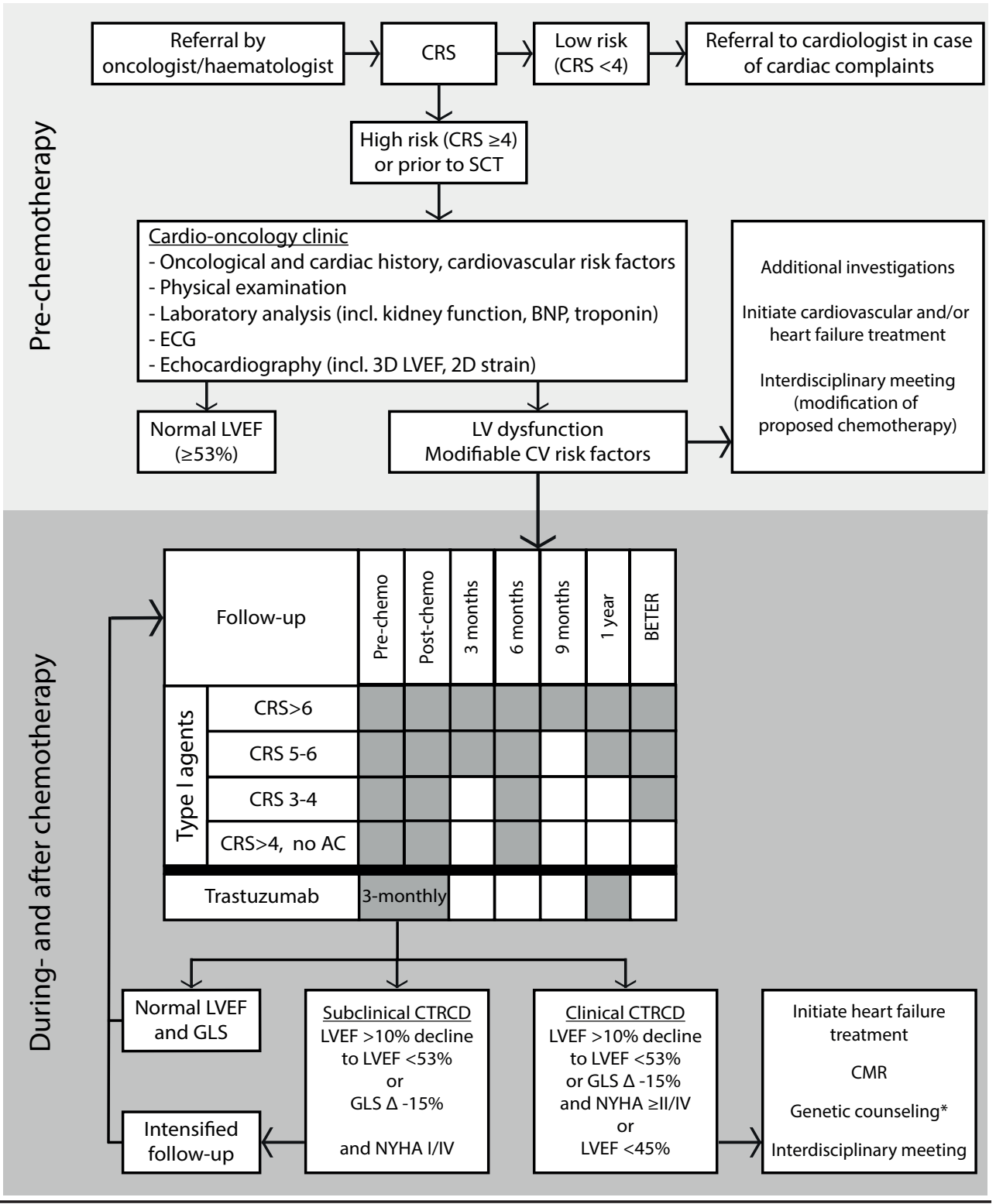

Figure 5 Cardio-oncology care at the University Medical Centre Utrecht, The Netherlands.

AC Anthracyclines; BNP brain natriuretic peptide; CMR cardiac magnetic resonance; CRS Cardiotoxicity Risk Score; CTRCD chemotherapy-related cardiac dysfunction; GLS global longitudinal strain; LVEF left ventricular ejection fraction; SCT stem-cell transplantation. * To be considered, depending on local policy 
If LV function has recovered, a re-challenge with the same chemotherapeutic agent(s) can be attempted under strict cardiological monitoring. Even though there are currently no evidence-based recommendations for heart failure medication in patients with this type of cardiotoxicity, treatment according to the ESC and ESMO guidelines should be considered if there is a persistent decline in LVEF and signs/symptoms of decompensation [9, 48].

\section{Subclinical cardiac dysfunction}

At this point in time, there is no evidence that initiation of heart failure medication in patients with subclinical cardiac dysfunction improves outcome. To prevent unnecessary treatment, we have decided to wait with initiation of heart failure treatment until the patient develops signs of clinical CTRCD, during the intensified follow-up.

Table 3 Ongoing trials on the treatment of cardiotoxicity

\begin{tabular}{|c|c|c|c|c|}
\hline Location & NCT Number & Intervention & Start date & Status \\
\hline United States of America & NCTo2943590 & Atorvastatin OR placebo & OI/20I7 & Recruiting \\
\hline United States of America & NCTo2674204 & Atorvastatin OR placebo & $05 / 2016$ & Recruiting \\
\hline United States of America & NCTo2096588 & Simvstatin OR placebo & $05 / 2014$ & $\begin{array}{l}\text { Active, not } \\
\text { recruiting }\end{array}$ \\
\hline Canada & NCT03186404 & Atorvastatin OR placebo & $07 / 2017$ & $\begin{array}{l}\text { Not yet } \\
\text { recruiting }\end{array}$ \\
\hline United Kingdom & NCT03265574 & Enalapril OR placebo & 09/20I7 & $\begin{array}{l}\text { Not yet } \\
\text { recruiting }\end{array}$ \\
\hline Italy & NCToI968200 & Enalapril & $\mathrm{I} 2 / 2 \mathrm{OI} 2$ & $\begin{array}{l}\text { Active, not } \\
\text { recruiting }\end{array}$ \\
\hline United States of America & NCTo2I77II75 & Carvedilol OR placebo & o6/20I4 & $\begin{array}{l}\text { Active, not } \\
\text { recruiting }\end{array}$ \\
\hline Brazil & NCToI724450 & Carvedilol OR placebo & $06 / 2012$ & Recruiting \\
\hline United States of America & NCTo27I7507 & Carvedilol OR placebo & 04/2016 & Recruiting \\
\hline United States of America & NCToI347970 & Carvedilol OR placebo & $05 / 2012$ & $\begin{array}{l}\text { Active, not } \\
\text { recruiting }\end{array}$ \\
\hline Italy & NCTo22368o6 & $\begin{array}{l}\text { Bisoprolol OR Ramipril OR } \\
\text { placebo }\end{array}$ & 07/20I5 & Recruiting \\
\hline Canada & NCToIoi6886 & $\begin{array}{l}\text { Perindopril OR Bisoprolol } \\
\text { OR placebo }\end{array}$ & 09/20I0 & $\begin{array}{l}\text { Active, not } \\
\text { recruiting }\end{array}$ \\
\hline United States of America & NCToIoo99i8 & $\begin{array}{l}\text { Carvedilol OR Lisinopril OR } \\
\text { placebo }\end{array}$ & 03/20IO & $\begin{array}{l}\text { Active, not } \\
\text { recruiting }\end{array}$ \\
\hline
\end{tabular}




\section{Future perspectives}

Despite the advantages in our understanding of this specific heart-failure entity with regard to the underlying pathophysiological mechanisms, improving diagnostic accuracy, and implementation of specific therapeutic interventions, there are still several unresolved issues and challenges within the field of cardio-oncology. To detect opportunities for improvement at this moment in time, the routinely provided cardiovascular care in oncology patients prior to the cardio-oncology era has to be investigated. This includes the frequency at which baseline cardiac function is assessed, the incidence of cardiovascular complications, referral patterns, treatment initiation- and response. We acknowledge that the (adjusted) CRS is probably insufficient to accurately identify high-risk patients. Development of sophisticated algorithms, which can be applied in the clinical field, will be an important focus of future trials and registries in order to optimise resources and pursue a cost-effective health care system. Risk stratification models need prospective validation and further improvement by the identification of additional (genetic) risk factors. Furthermore, personalised chemotherapeutic regimens, with their increasing complexity, go hand in hand with the need to establish interactions between agents and the combined effect on the cardiovascular system. Early detection of subclinical cardiac damage and dysfunction seems essential to optimise the treatment response rate; therefore, suitable biomarkers as well as the timing of biomarker sampling and echocardiographic monitoring need to be investigated. Ongoing therapeutic trials (Table 3) will shed more light on the potential of conventional heart failure treatment in this population as well as the optimal timing of treatment initiation. The establishing of specialised cardio-oncology units across the Netherlands will speed the development of this field, optimising the cost-benefit ratio of chemotherapeutic treatment with the potential to improve both oncological and cardiac outcome [I9, 55]. Furthermore, we will launch the ONCOR prospective multicentre registry in the near future, in which we aim to collect information on patients visiting cardio-oncology units across the Netherlands. Information from this registry will enable further national and international studies to improve the prognosis of this patient population. 


\section{References}

I de Moor JS, Mariotto AB, Parry C, et al. Cancer survivors in the United States: prevalence across the survivorship trajectory and implications for care. Cancer Epidemiol Biomarkers Prev. 2013;22:56I-570.

2 Verdecchia A, Francisci S, Brenner H, et al. Recent cancer survival in Europe: a 2000-02 period analysis of EUROCARE-4 data. Lancet Oncol. 2007;8:784-796.

3 Cardinale D, Colombo A, Bacchiani G, et al. Early detection of anthracycline cardiotoxicity and improvement with heart failure therapy. Circulation. 20I5;131:198I-I988.

4 Cardinale D. A new frontier: cardio-oncology. Cardiologia. 1996;41:887-89I.

5 Naaktgeboren WR, Linschoten M. de Graeff, et al. Cardiovascular health in adult cancer survivors. Maturitas. 2017:105:37-45.

6 Rochette L, Guenancia C, Gudjoncik A, et al. Anthracyclines/trastuzumab: new aspects of cardiotoxicity and molecular mechanisms. Trends Pharmacol Sci. 2015;36:326-348.

7 Lenneman CG, Cardio-oncology SDB. an update on cardiotoxicity of cancer-related treatment. Circ Res. 2016;II8:I008-IO20. doi: I0.II6I/CIRCRESAHA.II5.303633.

8 Moslehi JJ. Cardiovascular toxic effects of targeted cancer therapies. N Engl J Med. 2016;375:I457-I467.

9 Curigliano G, Cardinale D, Suter T, et al. Cardiovascular toxicity induced by chemotherapy, targeted agents and radiotherapy: ESMO Clinical Practice Guidelines. Ann Oncol. 2012;23:viiis5-viir66.

Io Christenson ES, James T, Agrawal V, Park BH. Use of biomarkers for the assessment of chemotherapyinduced cardiac toxicity. Clin Biochem. 2015;48(4-5:223-235.

II Thavendiranathan P, Poulin F, Lim KD, et al. Use of myocardial strain imaging by echocardiography for the early detection of cardiotoxicity in patients during and after cancer chemotherapy: a systematic review. J Am Coll Cardiol. 2014;63:275I-2768.

I2 Thavendiranathan P, Wintersperger BJ, Flamm SD, et al. Cardiac MRI in the assessment of cardiac injury and toxicity form cancer chemotherapy: a systematic review. Circ Cardiovasc Imaging. 2013;6:I080-I09I.

I3 Plana JC, Galderisis M, Barac A, et al. Expert consensus for multimodality imaging evaluation of adult patients during and after cancer therapy: a report from the American Society of Echocardiography and the European Association of Cardiovascular Imaging. J Am Soc Echocardiogr. 2014;27:9II-939.

I4 Herrmann J, Lerman A, Sandhu NP, Villarraga HR, Mulvagh SL, Kohli M. Evaluation and management of patients with heart disease and cancer: cardio-oncology. Mayo Clin Proc. 20I4;89:1287-I306.

I5 Zamorano JL, Lancellotti P, Rodriguez Muñoz D, et al. 20I6 ESC Position Paper on cancer treatments and cardiovascular toxicity developed under the auspices of the ESC Committee for Practice Guidelines: The Task Force for cancer treatments and cardiovascular toxicity of the European Society of Cardiology (ESC) Eur Heart J. 2016;37:2768-280I.

I6 Lancellotti P, Nkomo VT, Badano LP, et al. Expert consensus for multi-modality imaging evaluation of cardiovascular complications of radiotherapy in adults: a report from the European Association of Cardiovascular Imaging and the American Society of Echocardiography. J Am Soc Echocardiogr. 2013;26:IOI3-I032.

I7 van Dalen EC, Caron HN, Dickinson HO, Kremer LC. Cardioprotective interventions for cancer patients receiving anthracyclines. Cochrane Database Syst Rev. 2011;6:CDoo39I7.

I8 Kalam K, Marwick TH. Role of cardioprotective therapy for prevention of cardiotoxicity with chemotherapy: a systematic review and meta-analysis. Eur J Cancer. 2013;49:2900-2909.

I9 Johnson MN, Steingart R, Carver J. How to develop a cardio-oncology fellowship. Heart Fail Clin. 2017:13:36I-366.

20 Lotrionte M, Biondi-Zoccai G, Abbate A, et al. Review and meta-analysis of incidence and clinical predictors of anthracycline cardiotoxicity. Am J Cardiol. 2013;II2:I980-I984.

2I Yeh ETH. Cardiovascular complications of cancer therapy: diagnosis, pathogenesis, and management. Circulation. 2004;109:3122-313I.

22 Felker GM, Thompson RE, Hare JM, et al. Underlying causes and long-term survival in patients with initially unexplained cardiomyopathy. N Engl J Med. 2000;342:I077-I084.

23 Von Hoff DD, Layard MW, Basa P, et al. Risk factors for doxorubicin-induced congestive heart failure. Ann Intern Med. 1979;9I:710-717.

24 Ewer MS, Ewer SM. Cardiotoxicity of anticancer treatments. Nat Rev Cardiol. 2015;12:547-558. 
25 Minotti G, Menna P, Salvatorelli E, Cairo G, Anthracyclines GL. molecular advances and pharmacologic developments in antitumor activity and cardiotoxicity. Pharmacol Rev. 2004;56:185-229.

26 Rafiyath SM, Rasul M, Lee B, Wei G, Lamba G, Liu D. Comparison of safety and toxicity of liposomal doxorubicin vs. conventional anthracyclines: a meta-analysis. Exp Hematol Oncol. 20I2;1:IO.

27 van Dalen EC, van der Pal HJ, Kremer LC. Different dosage schedules for reducing cardiotoxicity in people with cancer receiving anthracycline chemotherapy. Cochrane Database Syst Rev. 2016;3:CDoo5008.

28 Slamon DJ, Leyland-Jones B, Shak S, et al. Use of chemotherapy plus a monoclonal antibody against HER2 for metastatic breast cancer that overexpresses HER2. N Engl J Med. 200I;344:783-792.

29 Nohria A. Prevention of cardiomyopathy in patients with cancer. JACC online: http://www.acc.org/ latest-in-cardiology/articles/2016/09/29/13/25/prevention-of-cardiomyopathy-in-patients-with-cancer Consulted on I4 March 20I7.

30 Senkus E, Kyriakides S, Ohno S, et al. Primary breast cancer: ESMO clinical practice guidelines for diagnosis, treatment and follow-up. Ann Oncol. 2015;5:8-30.

3I Mantarro S, Rossi M, Bonifazi M, et al. Risk of severe cardiotoxicity following treatment with trastuzumab: a meta-analysis of randomized and cohort studies of 29,000 women with breast cancer. Intern Emerg Med. 2016;II:I23-I40.

32 Ewer MS, Vooletich MT, Durand JB, et al. Reversibility of trastuzumab-related cardiotoxicity: new insights based on clinical course and response to medical treatment. J Clin Oncol. 2005;23:7820-7826.

33 Bloom MW, Hamo CE, Cardinale D, et al. Cancer therapy-related cardiac dysfunction and heart failure: Part I: definitions, pathophysiology, risk factors, and imaging. Circ Heart Fail. 2016;9:e00266I.

34 Mushlin PS, Cusack BJ, Boucek RJ, Jr, Andrejuk T, Li X, Olson RD. Time-related increases in cardiac concentrations of doxorubicinol could interact with doxorubicin to depress myocardial contractile function. Br J Pharmacol. 1993;110:975-982.

35 Zhang S, Liu X, Bawa-Khalfe T, et al. Identification of the molecular basis of doxorubicin-induced cardiotoxicity. Nat Med. 2012;18:1639-I642.

36 De Keulenaer GW, Doggen K, Lemmens K. The vulnerability of the heart as a pluricellular paracrine organ: lessons from unexpected triggers of heart failure in targeted ErbB2 anticancer therapy. Circ Res. 2010;106:35-46.

37 Telli ML, Hunt SA, Carlson RW, Guardino AE. Trastuzumab-related cardiotoxicity: calling into question the concept of reversibility. J Clin Oncol. 2007;25:3525-3533.

38 Krischer JP, Epstein S, Cuthbertson DD, Goorin AM, Epstein ML, Lipshultz SE. Clinical cardiotoxicity following anthracycline treatment for childhood cancer: the Pediatric Oncology Group experience. J Clin Oncol. 1997;15:1544-I552.

39 Linschoten M, Teske AJ, Cramer MJ, van der Wall E, Asselbergs FW. Chemotherapy-related cardiac dysfunction - a systematic review of genetic variants modulating individual risk. Circ Genom Precis. Med. 2018;II:eool753.

40 Dranitsaris G, Rayson D, Vincent M, et al. The development of a predictive model to estimate cardiotoxic risk for patients with metastatic breast cancer receiving anthracyclines. Breast Cancer Res Treat. 2008;107:443-450.

4I Ezaz G, Long JB, Gross CP, Chen J. Risk prediction model for heart failure and cardiomyopathy after adjuvant trastuzumab therapy for breast cancer. J Am Heart Assoc. 20I4;3:e000472.

42 Cardinale D, Biasillo G, Salvatici M, Sandri MT, Cipolla CM. Using biomarkers to predict and to prevent cardiotoxicity of cancer therapy. Expert Rev Mol Diagn. 2017;17:245-256.

43 Gottdiener JS, Mathisen DJ, Borer JS, et al. Doxorubicin cardiotoxicity: assessment of late left ventricular dysfunction by radionuclide cineangiography. Ann Intern Med. 1981;94:430-435.

44 Breast Cancer Guideline version 2.0, NABON 20I2: http:/www.oncoline.nl/uploaded/docs/ mammacarcinoom/Dutch\%2oBreast\%20Cancer\%20Guideline\%2020I2.pdf Consulted on 30th of March 20I7.

45 Thavendiranathan P, Grant AD, Negishi T, et al. Reproducibility of echocardiographic techniques for sequential assessment of left ventricular ejection fraction and volumes: application to patients undergoing cancer chemotherapy. J Am Coll Cardiol. 2013;6I:77-84. 
46 Teske AJ, De Boeck BW, Melman PG, Sieswerda GT, Doevendans PA, Cramer MJ. Echocardiographic quantification of myocardial function using tissue deformation imaging, a guide to image acquisition and analysis using tissue Doppler and speckle tracking. Cardiovasc Ultrasound. Acta Neurochir (Wien) 2007;5:27.

47 Grothues F, Smith GC, Moon JC, et al. Comparison of interstudy reproducibility of cardiovascular magnetic resonance with two-dimensional echocardiography in normal subjects and in patients with heart failure or left ventricular hypertrophy. Am J Cardiol. 2002;90:29-34.

48 Ponikowski P, Voors AA, Anker SD, et al. 20I6 ESC Guidelines for the diagnosis and treatment of acute and chronic heart failure: The Task Force for the diagnosis and treatment of acute and chronic heart failure of the European Society of Cardiology (ESC). Developed with the special contribution of the Heart Failure Association (HFA) of the ESC. Eur J Heart Fail. 2016;18:89I-975.

49 Cardinale D, Colombo A, Lamantia G, et al. Anthracycline-induced cardiomyopathy: clinical relevance and response to pharmacologic therapy. J Am Coll Cardiol. 2010;55:213-220.

50 Avila MS, Ayub-Ferreira SM, de Barros Wanderley MR, Jr, et al. Carvedilol for prevention of chemotherapy-related cardiotoxicity: the CECCY trial. J Am Coll Cardiol. 2018;71:228I-2290.

5I Boekhout AH, Gietema JA, Milojkovic Kerklaan B, et al. Angiotensin II-receptor inhibition with candesartan to prevent trastuzumab-related cardiotoxic effects in patients with early breast cancer: a randomized clinical trial. Jama Oncol. 2016;2:1030-1037.

52 Dekker N, van 't Veer MB, Aleman BM, et al. The BETER survivorship care initiative for Hodgkin lymphoma; a tailored survivorship care for late effects of treatment. Ned Tijdschr Geneeskd. 2015;159:A9269.

53 Wasielewski M, van Spaendonck-Zwarts KY, Westerink ND, et al. Potential genetic predisposition for anthracycline-associated cardiomyopathy in families with dilated cardiomyopathy. Open Heart. 20I4;: :eoooli6.

54 Linschoten M, Teske AJ, Baas AF, et al. Truncating titin (TTN) variants in chemotherapy-induced cardiomyopathy. J Card Fail. 2017;23:476-476.

55 Shee K, Kono AT, D'Anna SP, et al. Maximizing the benefit-cost ratio of anthracyclines in metastatic breast cancer: case report of a patient with a complete response to high-dose doxorubicin. Case Rep Oncol. 2016;9:840-846. 


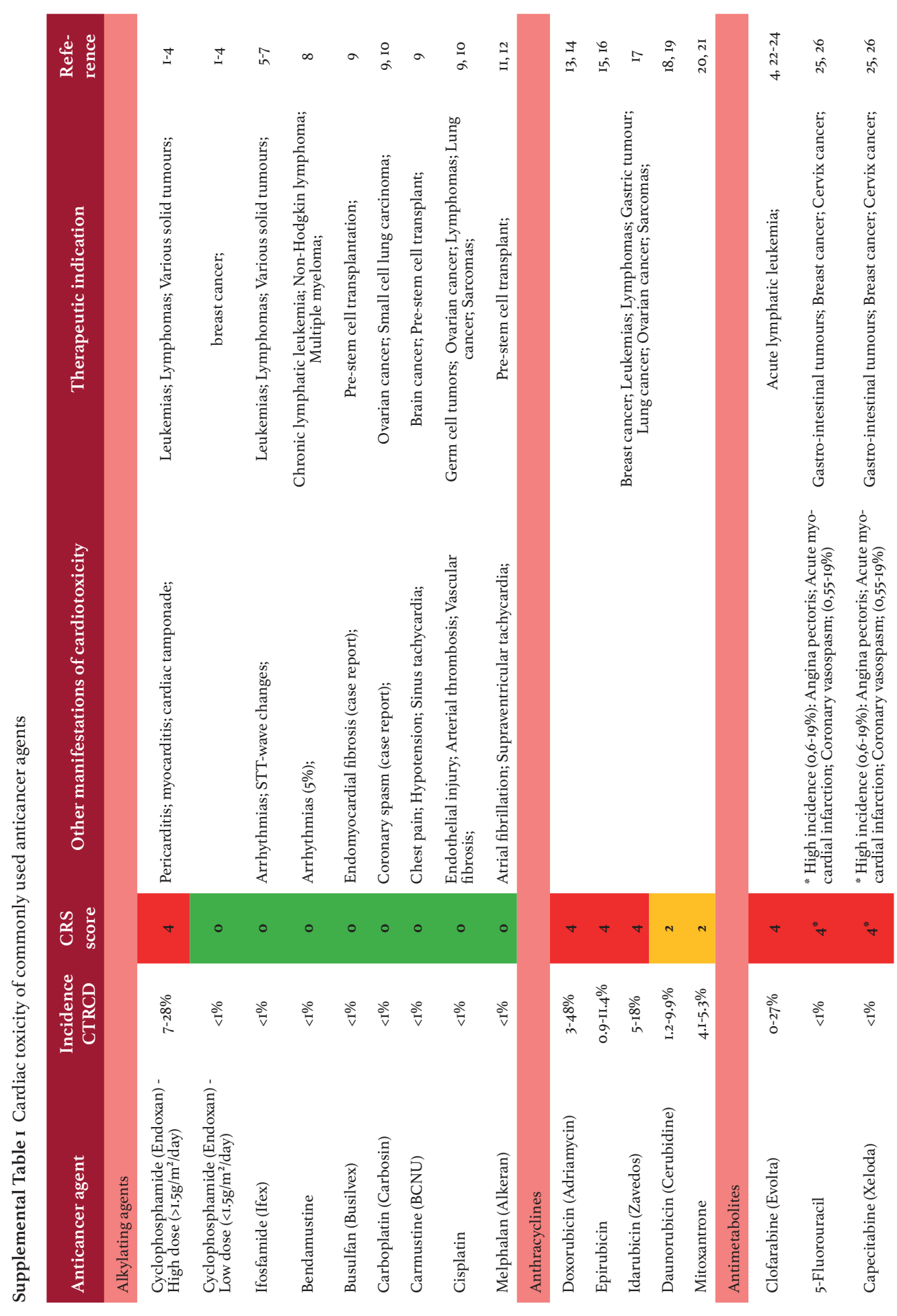




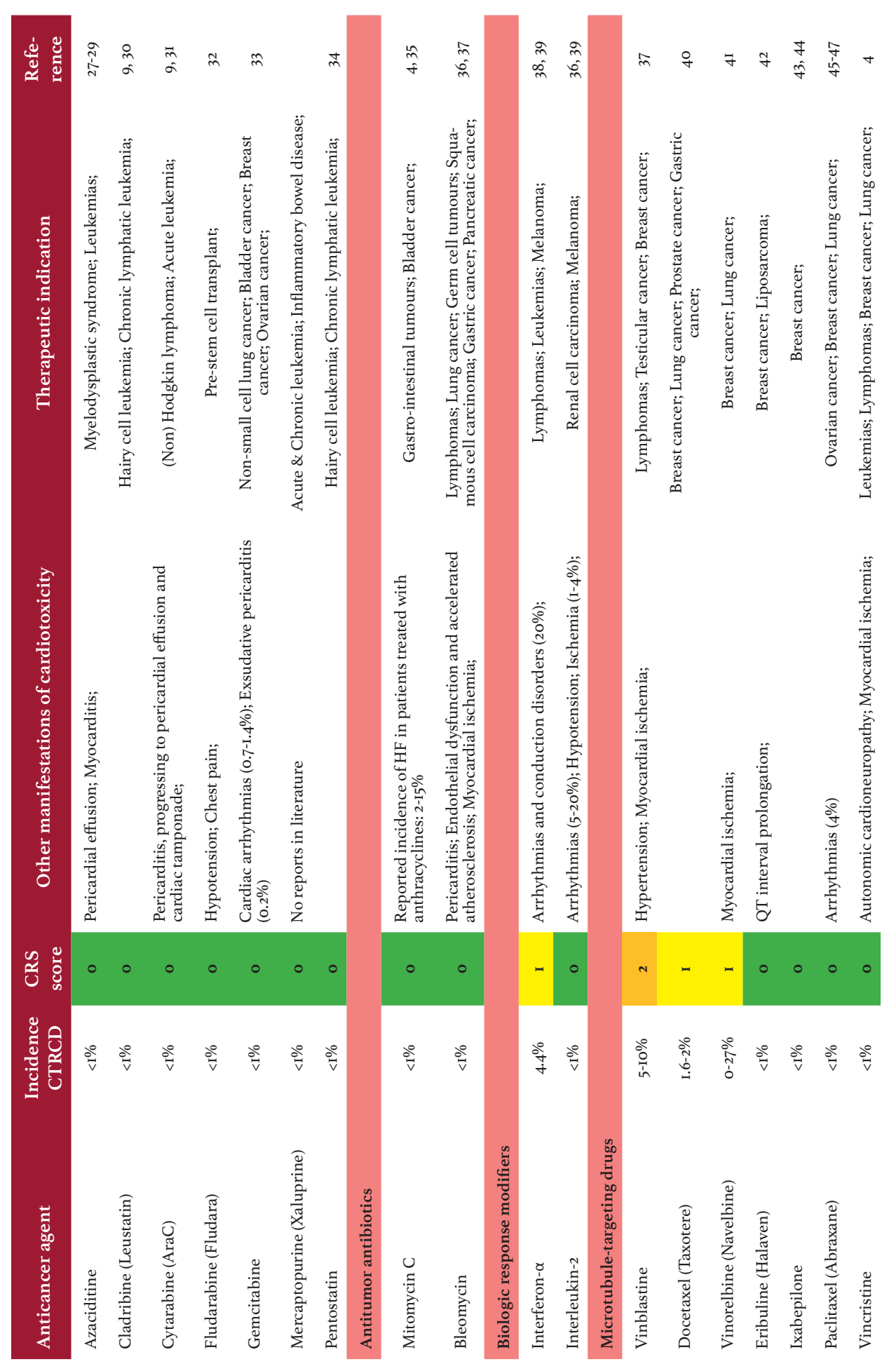




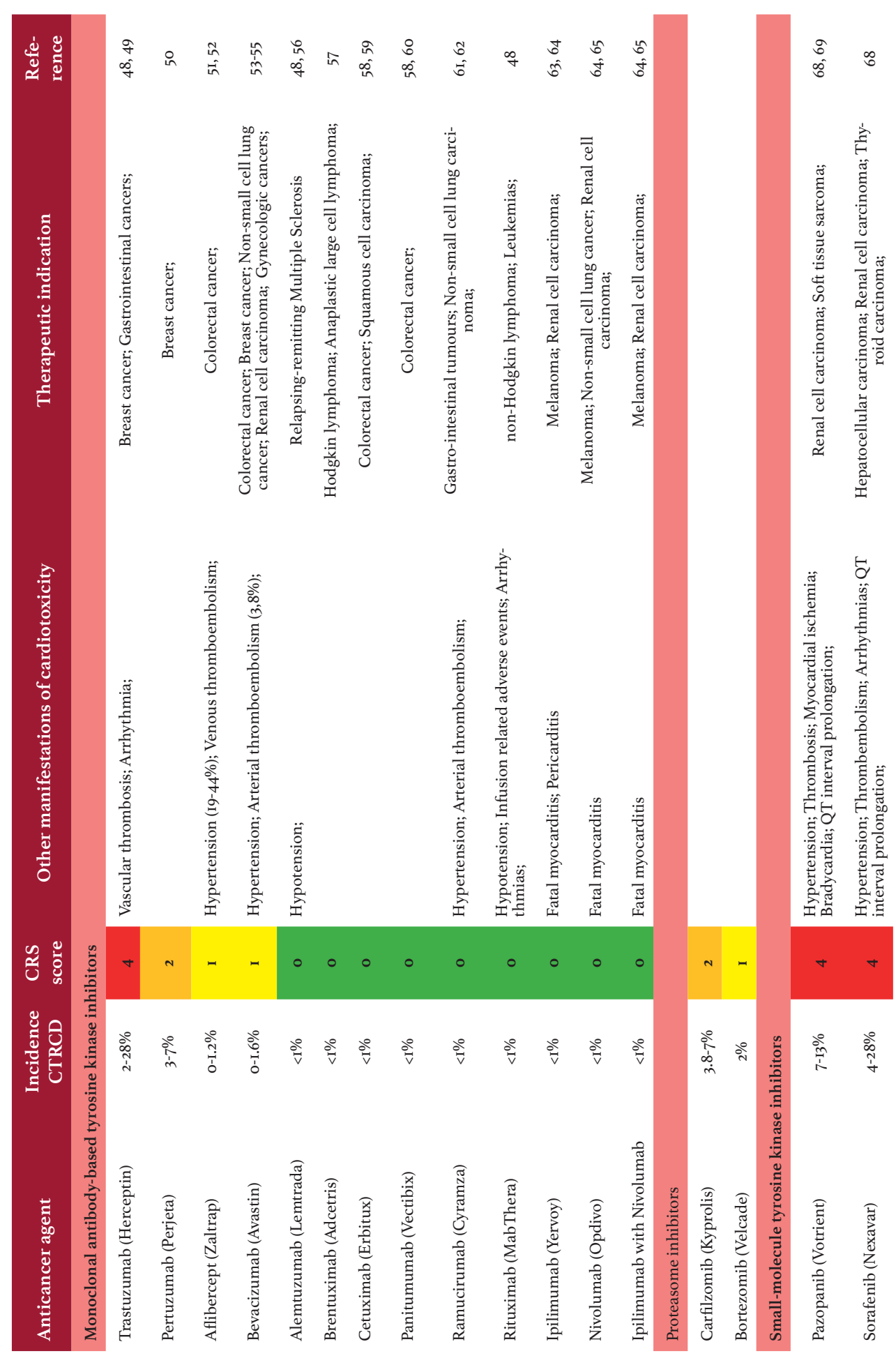




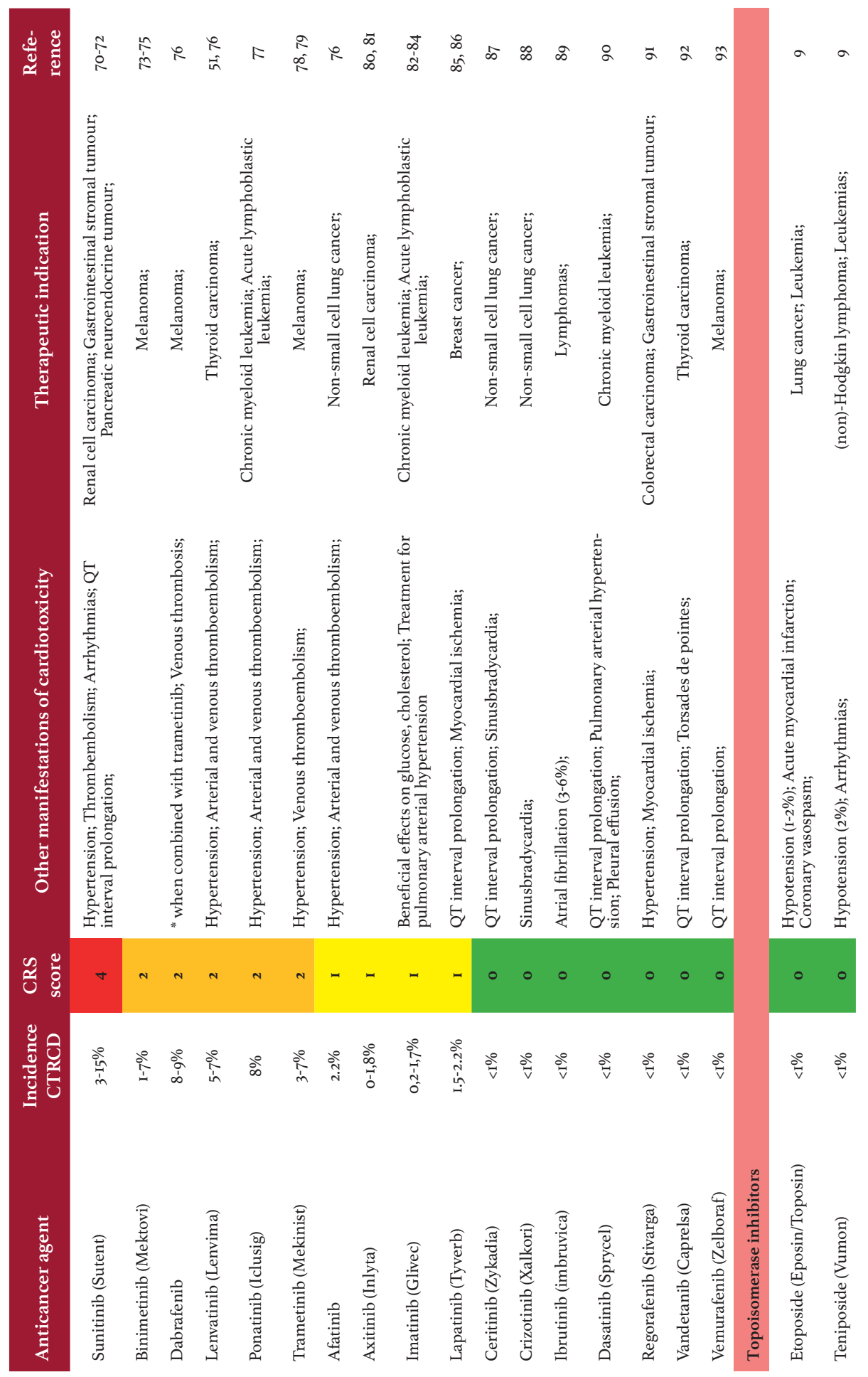




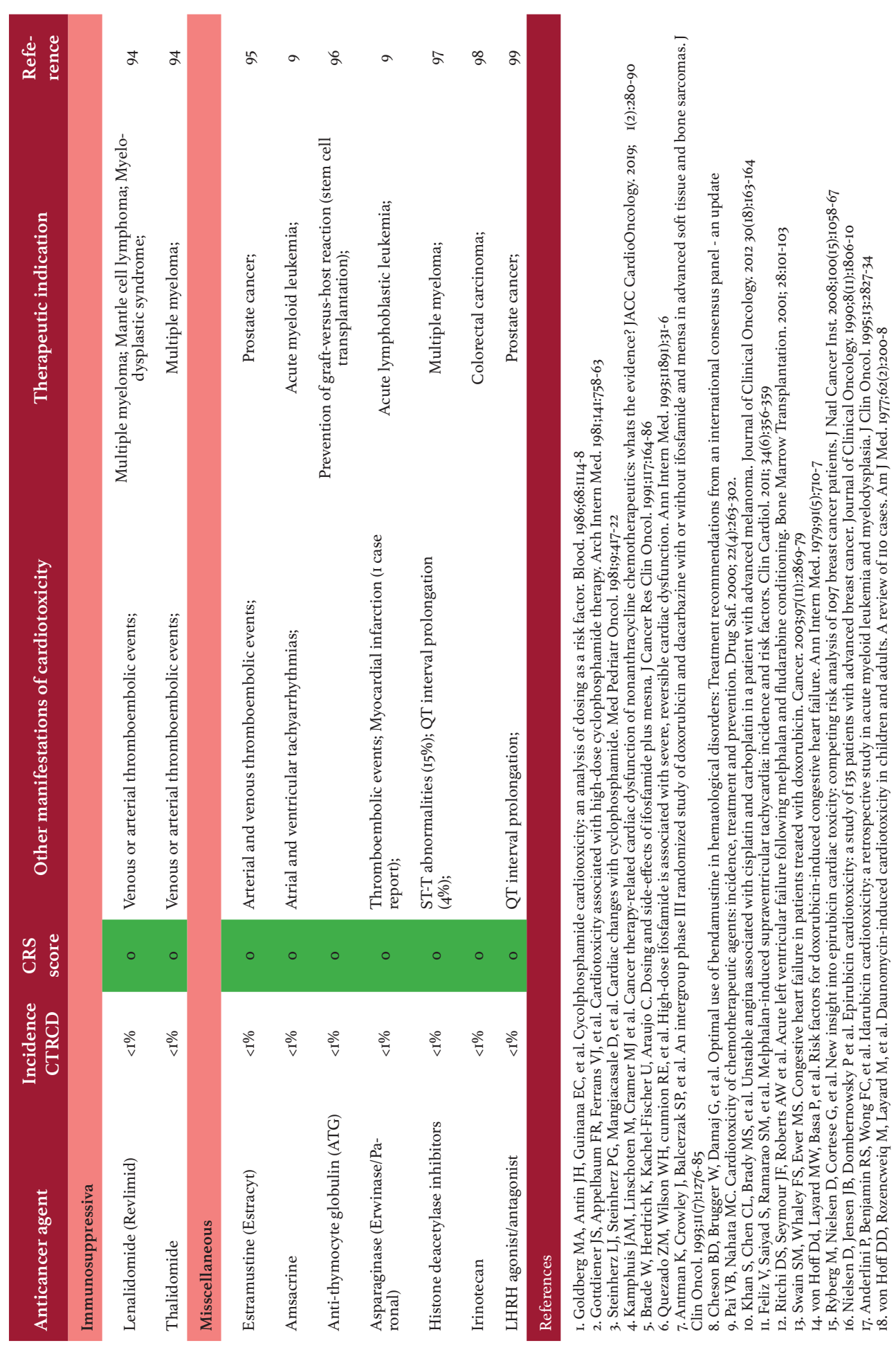




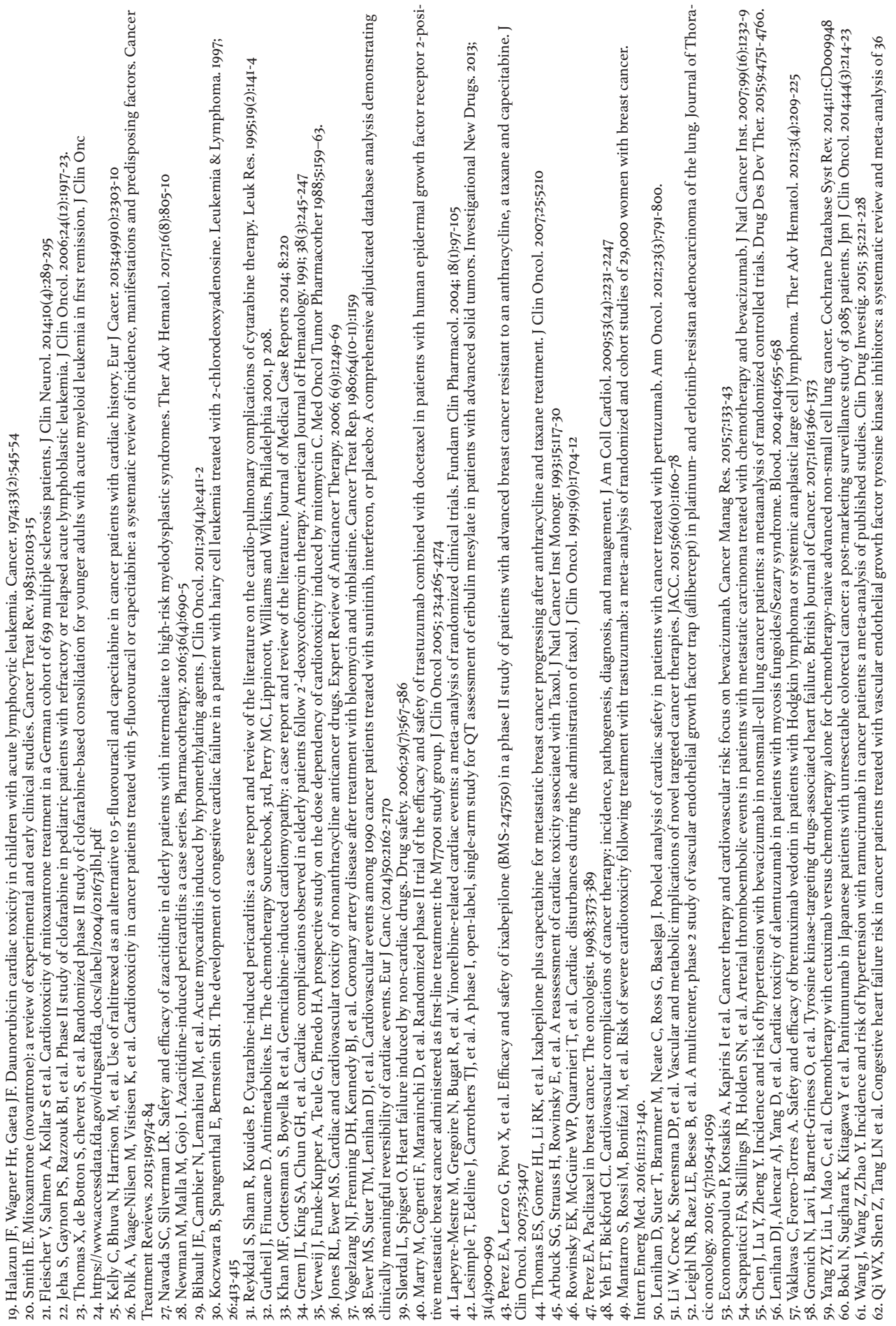



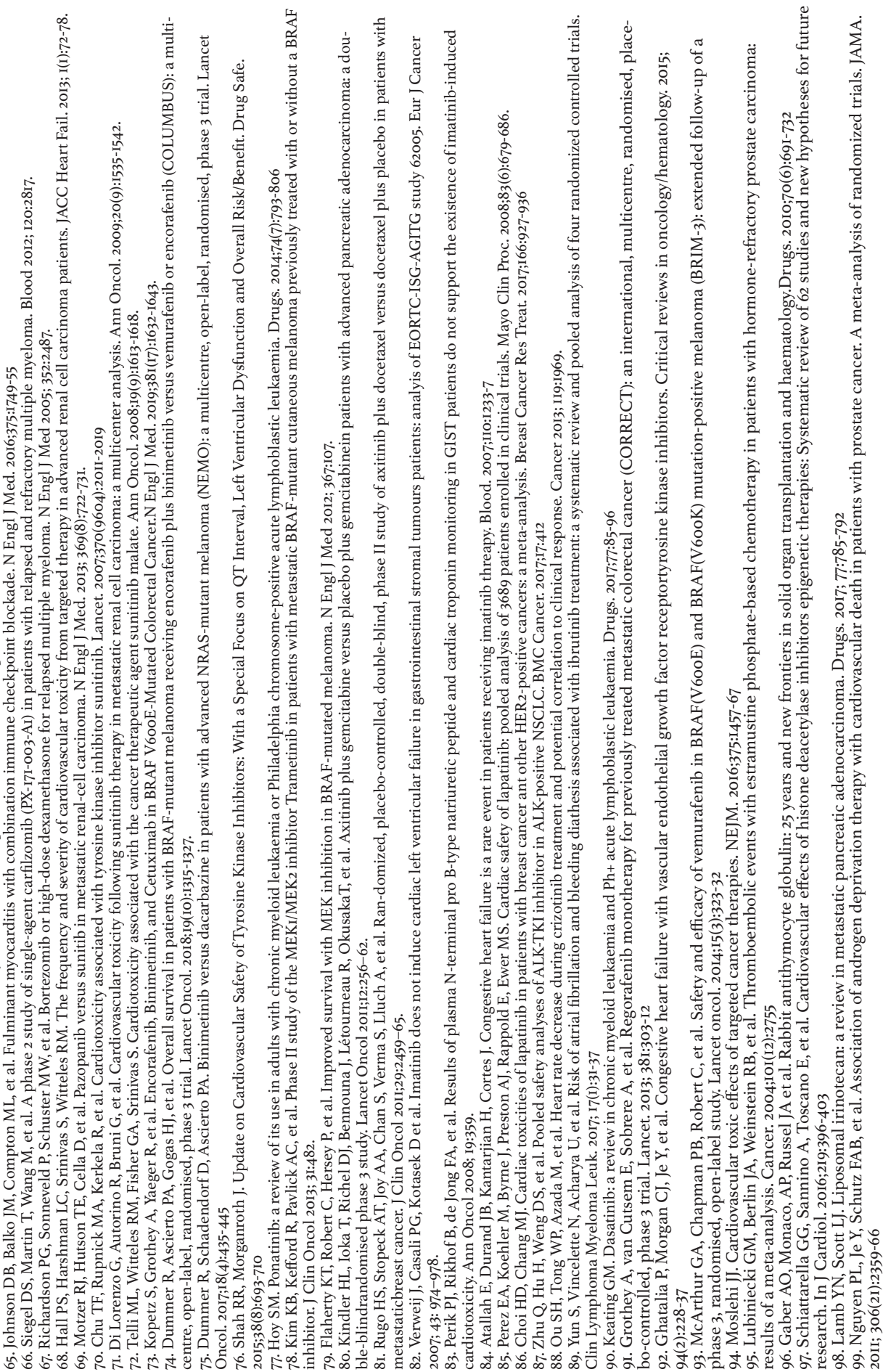
Chapter 3

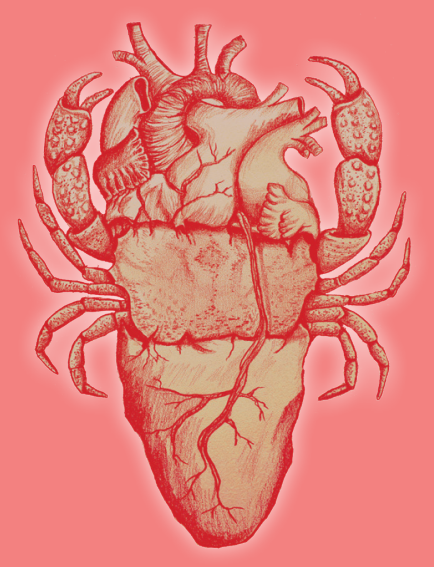




\section{Cancer therapy-related cardiac dysfunction of non-anthracycline chemotherapeutics What's the evidence?}

JACC CardioOncology 20I9; I(2):280-9o

JAM Kamphuis ${ }^{1}$, M Linschoten ${ }^{\mathrm{I}}$, MJ Cramer ${ }^{\mathrm{I}}$, EH Gort $^{2}$, A van Rhenen ${ }^{3}$, FW Asselbergs ${ }^{1,4,5}$, PA Doevendans ${ }^{1,6}$, AJ Teske ${ }^{\mathrm{I}}$

I Department of Cardiology, Division of Heart and Lungs, University Medical Centre Utrecht, Utrecht, The Netherlands 2 Department of Medical Oncology, University Medical Centre Utrecht, Utrecht, The Netherlands 3 Department of Haematology, University Medical Centre Utrecht, Utrecht, The Netherlands 4 Institute of Cardiovascular Science, Faculty of Population Health Sciences, University College London, London, UK 5 Health Data Research UK and Institute of Health Informatics, University College London, London, UK 6 Netherlands Heart Institute, Utrecht, The Netherlands 


\begin{abstract}
Cancer therapy-related cardiac dysfunction (CTRCD) is one of the most concerning cardiovascular side effects of cancer treatment. Important reviews within the field of cardio-oncology have described various agents to be associated with a high risk of CTRCD, including mitomycin-C, ifosfamide, vincristine, cyclophosphamide and clofarabine. The aim of this study is to provide insight into the data upon which these incidence rates are based. We observed that the reported cardiotoxicity of mitomycin- $\mathrm{C}$ and ifosfamide is based on studies in which most patients received anthracyclines, complicating the interpretation of their association with CTRCD. The high incidence of vincristine-induced cardiotoxicity is based on an incorrect interpretation of a single study. Incidence rates of clofarabine remain uncertain due to a lack of cardiac screening in clinical trials. Administration of high-dose cyclophosphamide ( $>\mathrm{I} .5 \mathrm{~g} / \mathrm{m}^{2} / \mathrm{day}$ ) is associated with a high incidence of CTRCD. Based on our findings, a critical re-evaluation of the cardiotoxicity of these agents is warranted.
\end{abstract}




\section{Introduction}

In recent years, considerable attention by cardiologists and oncologists worldwide has been devoted to decreasing the adverse cardiovascular side effects of cancer treatment. The position paper of the European Society of Cardiology on cancer treatment and cardiovascular toxicity further increased awareness of the new discipline "cardio-oncology"[I]. Cardiovascular toxicity of anticancer treatment can manifest itself in various ways, including hypertension, arrhythmias, pericarditis, and coronary artery disease. One of the most concerning side effects is cancer therapy-related cardiac dysfunction (CTRCD), typically defined by declines in left ventricular ejection fraction with or without symptoms of heart failure (HF). The development of CTRCD is dependent on patient-related factors such as age and pre-existing cardiovascular disease but also specifically on the chemotherapeutic agent. Anthracyclines are notorious for causing cardiomyocyte damage in a dose-dependent manner. The incidence of doxorubicin-related HF is estimated at $5 \%, 16 \%, 26 \%$, and $48 \%$ for cumulative doses of $400,500,550$, and $700 \mathrm{mg} / \mathrm{m}^{2}$, respectively [2]. Patients who develop cardiac dysfunction after anthracycline administration carry a prognosis similar to that of idiopathic dilated cardiomyopathy, with a 5- and Io-year cardiovascular mortality rate of $9 \%$ and $24 \%$, respectively [3]. Another agent of which CTRCD has been studied extensively is the monoclonal antibody trastuzumab (Herceptin ${ }^{\circledR}$ ). In contrast to anthracycline-induced cardiac dysfunction, trastuzumab-related cardiac dysfunction is not dose dependent and is reversible in most cases [4]. Because there may be a time-dependent relationship between HF treatment initiation and recovery of cardiac function [5], proper risk stratification is key in facilitating the early detection and treatment of this side effect. In addition to anthracyclines, mitoxantrone, and trastuzumab, recent important review articles within the field of cardio-oncology and the European Society of Cardiology position paper have reported various other chemotherapeutic agents as highly cardiotoxic (i.e., $\geq$ IO\% incidence of CTRCD) (Table I). The purpose of this study was to investigate the origin of the currently used incidence rates of CTRCD in these frequently cited articles (Table I) $[\mathrm{I}, 4,6,7]$.

\section{Methods}

Four landmark review articles within the field of cardio-oncology that reported the incidence of CTRCD were used to identify nonanthracycline agents, which have been described as highly cardiotoxic (i.e., causing CTRCD in $\geq 10 \%$ of patients treated) $[\mathrm{I}, 4,6,7]$. Agents that were classified as "highly cardiotoxic" in $\geq 2$ of these 4 review articles were included in our subsequent analysis. This resulted in the inclusion of the following 5 chemotherapeutics: mitomycin C (MMC), vincristine, clofarabine, ifosfamide, and cyclophosphamide (Table I). We thoroughly studied the articles referenced by these review articles and evaluated the incidence rates, definitions of CTRCD, and prior or concurrent use of other known cardiotoxic anticancer agents, including anthracyclines and the anthraquinone mitoxantrone. Additionally, we searched for other trials describing the cardiotoxic side effects of the selected agents. For clofarabine, we performed a systematic literature review using the search term "clofarabine" and applying the filter "clinical trials." This search yielded 98 
studies in total, including 13 clinical trials in which clofarabine was used as a first-line agent. For the other agents, it was out of the scope of this primer to perform a systematic review, considering the large body of literature published on these agents ( $M M C, n=2$, IOO; cyclophosphamide, $n$ > I0,000; ifosfamide, $n=1,636$; vincristine, $n=4,499$ ).

\section{Results}

\section{Mitomycin-C}

MMC is an alkylating agent, that causes cross-linking of DNA and thereby inhibits DNA synthesis. It is used in the treatment of gastrointestinal-, genitourinary- and gynecological cancers. Two review articles describe that this chemotherapeutic leads to CTRCD in IO\% of patients [4,6], a number which was derived from a study by Verweij, et al. (1988) [8]. Verweij, et al. evaluated the incidence of $\mathrm{HF}$ in 37 patients treated with MMC and found I patient who developed cardiac failure after concomitant treatment with MMC and doxorubicin. This study found a frequency of HF of IO\% through pooling of their results with the results of 4 other clinical trials. The incidence of HF in these latter studies, in which at least $95 \%$ of the patients of each study population were also treated with doxorubicin [range IOO-80omg/m2], varied from 2.2-I5.4\% (Table I)[9-I2]. All patients that developed HF had been treated with anthracyclines as well, which complicates the interpretation of the true cardiotoxicity of MMC alone. Possible synergistic cardiotoxic effects between doxorubicin and MMC have been suggested by 2 of these studies [9, I2]. In the largest of these studies ( $\mathrm{n}=\mathrm{I} 80$ ), I4/9I (I5.4\%) patients treated with MMC and prior doxorubicin developed symptomatic HF, compared to $3 / 89$ (3.4\%) in patients treated only with doxorubicin [9].

The dose-dependent relationship $\left(>30 \mathrm{mg} / \mathrm{m}^{2}\right)$ with the incidence of CTRCD is also derived from the article of Verweij et al., and is based on a single patient who developed acute heart failure after administration of doxorubicin $\left(150 \mathrm{mg} / \mathrm{m}^{2}\right)$ and $\mathrm{MMC}(30 \mathrm{mg} /$ $\left.\mathrm{m}^{2}\right)$ [8]. Other clinical studies evaluating cardiotoxicity with MMC ( $\left.=198\right)$ have not described incident HF [13-I7]. More specifically, in one study whereby active cardiac screening was performed in 60 patients treated with MMC in combination with methotrexate and mitoxantrone, 2 patients developed asymptomatic LVEF declines (3.3\%) [14].

\section{Vincristine}

Vincristine (Oncovin) is a vinca-alkaloid that has been used since the I960s. It is an anti-mitotic, and disrupts cell division by interacting with tubulin proteins. Vincristine is included in treatment regimens for a variety of malignancies, including hematologic malignancies, primary brain tumors, sarcomas and pediatric tumors. The main toxic effect of vincristine is neurotoxicity, most frequently presenting as peripheral neuropathy and sometimes as autonomic neuropathy, affecting blood pressure control and heart rate variability [I8-I9].

The incidence of CTRCD is reported to be up to $25 \%$ in patients treated with this agent (Table I) $[4,6]$. However, this number is derived from an autopsy study by Roberts, et al. (1968), which described the incidence of cardiac tumors in 196 patients who died of 
CENTRAL ILLUSTRATION The time span of the introduction of chemotherapeutics and the publication of their cardiotoxic effects

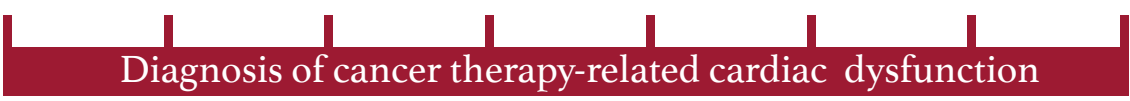

Heart failure symptoms

LVEF measurements

Strain analysis

Biomarkers
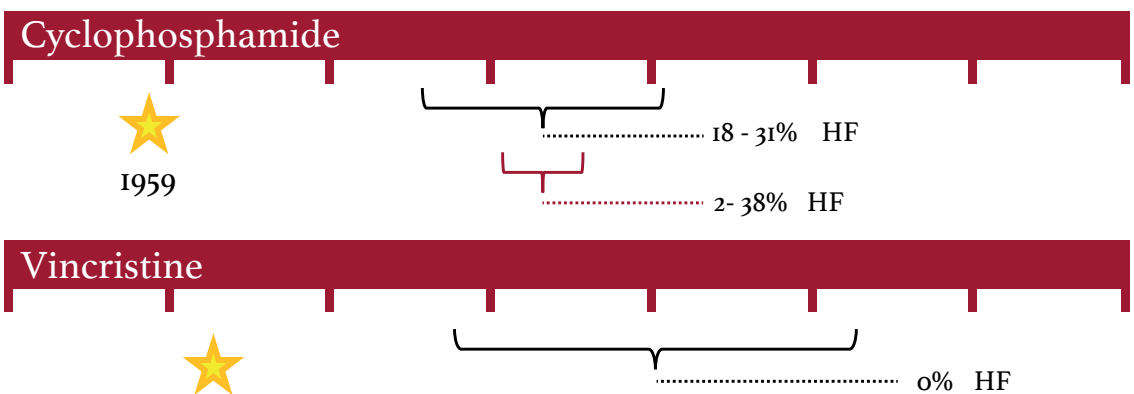

I963
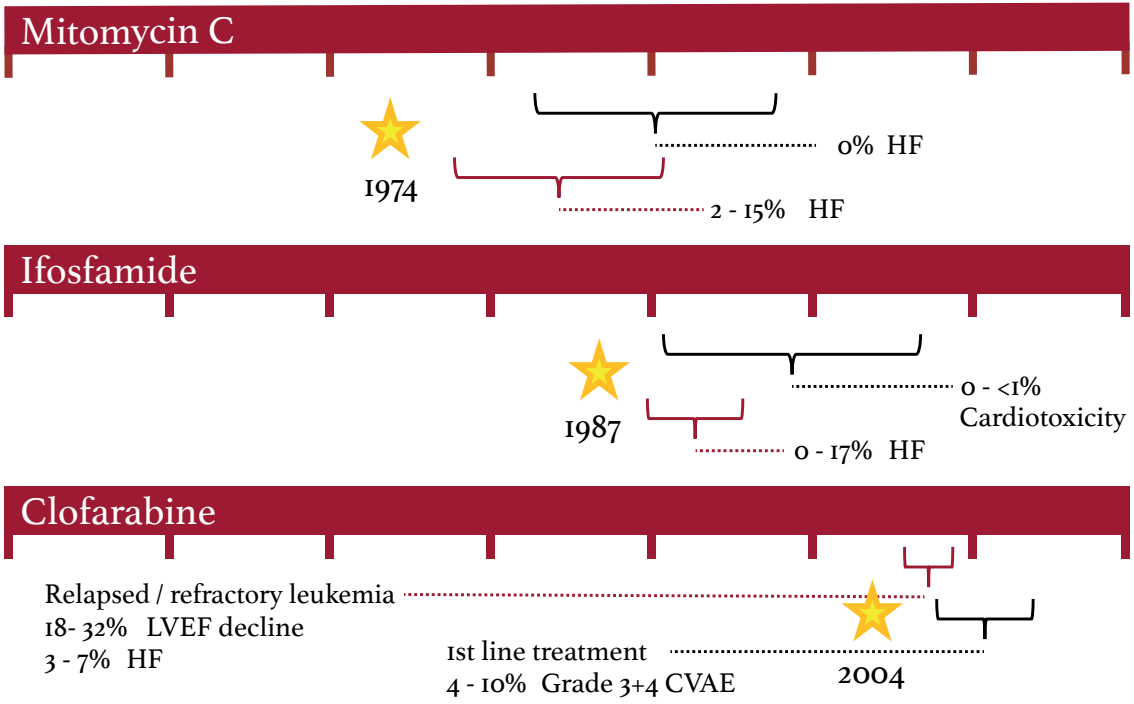

The time span of the introduction of chemotherapeutics (cyclophosphamide, vincristine, mitomycin C, ifosfamide, and clofarabine), methods for the detection of CTRCD, and the publication of their cardiotoxic effects. The brackets represent the time span in which the data were published, with a division between studies in which patients received prior or concurrent anthracyclines (red brackets) or treatment without anthracyclines (black brackets). The ranges are derived from the study outcomes from Table I. The incidence of Common Terminology Criteria for Adverse Events grade 3 and 4 cardiovascular adverse events (CVAEs) of first-line clofarabine treatment was based on only the studies that reported on CVAEs. 
malignant lymphoma [20]. They found cardiac involvement in 48 patients (24\%), of which 5 had clinical manifestations. This study did not report on cardiovascular side effects of treatment with vincristine. The suggested incidence of CTRCD of vincristine of $25 \%$ therefore seems to be an incorrect interpretation of this single study. A few case reports, referred to by Pai, et al. [6], have described cardiovascular side effects such as coronary spasm $(n=2)$ $[21,22]$ and myocardial infarction $(n=2)[23,24]$.

Treatment with vincristine monotherapy is uncommon, which makes it difficult to define the cardiotoxic risk of this specific agent. Several studies with vincristine monotherapy have not reported any cardiovascular side effects [25,26]. It has even been suggested that vincristine may have a protective effect on cardiomyocytes subjected to oxidative stress, which is hypothesized to be the underlying mechanism of anthracycline cardiotoxicity $[27,28]$. This finding was derived from animal studies, and has not been reproduced in human studies.

\section{Clofarabine}

Clofarabine is a relative new drug which was approved by the U.S. Food and Drug Administration (FDA) in 2004. This purine nucleoside antimetabolite has an antineoplastic effect by directly inhibiting DNA synthesis and ribonucleotide reductase and inducing apoptosis [29]. Clofarabine was initially used in patients with recurrent or refractory acute leukemia and more recently it was also incorporated in first-line regimens in acute leukemia patients. The incidence of left ventricular systolic dysfunction after administration of clofarabine has been reported to be $27 \%$ ( $15 / 55$ patients)(Table $\mathrm{I}$ ) $[\mathrm{I}, 4,6,7]$. This number is derived from the FDA approval letter [30], which described two studies of 96 pediatric patients with relapsed or refractory leukemia, all of who had prior treatment with other potentially cardiotoxic agents [31,32]. Cardiac assessment pre- and posttreatment was available in 68 patients. Pericardial effusion was seen in 23 of these 68 patients (34\%), although the extent of fluid was limited without any hemodynamic consequences in a majority of cases. A decrease in left ventricular systolic function was noted in 16 patients (24\%), 3 of which had signs of HF. In some patients these cardiac changes were transient in nature, although numbers were not specified. Since all patients received prior therapy with other cardiotoxic agent including anthracyclines, the role of clofarabine in provoking these cardiac abnormalities remains unclear.

Although the FDA has recommended serial cardiac assessment during clofarabine treatment, this is not routinely done. In our systematic literature search, we identified only 2 out of 13 trials [33-45] that included cardiac screening during and after therapy $[43,44]$. In one of these studies, cardiac function was only monitored in 5 out of 70 patients [43], and the other study did not report on cardiac outcomes despite active cardiac screening [44]. Of the II studies that did not perform systematic monitoring of LV function, 3studies did report on the occurrence of Common Terminology Criteria for Adverse Events (CTCAE) grade III-IV cardiac toxicity (arrhythmia 4\% [36]; overall Io\% [39]; overall $9 \%[4 \mathrm{I}])$. The widespread lack of cardiac screening and lack of consistent reporting of adverse cardiovascular events in clinical trials with clofarabine might imply that clofarabine is not associated with severe, clinical cardiotoxicity. However, the incidence of subclinical cardiotoxicity including an asymptomatic decline in left ventricular function remains uncertain. 


\section{Ifosfamide}

Ifosfamide is used in the treatment of hematological malignancies and sarcomas and belongs to the group of alkylating agents similar to MMC. The incidence of CTRCD is reported to be $0.5-17 \%$ after ifosfamide administration (Table $\mathrm{I}$ ) [I,4,6,7]. The highest incidence rate $(17 \%)$ is based on a study of Quezado, et al., where 9 cases of HF were retrospectively identified from a group of 52 patients who were treated with a single cycle high dose ifosfamide (IO-I $8 \mathrm{~g} / \mathrm{m}^{2} /$ cycle) [46]. However, all patients received prior treatment with anthracyclines in doses ranging from 190 to $550 \mathrm{mg} / \mathrm{m}^{2}$, which raises the question of at least a partial contribution of anthracyclines to the development of CTRCD. A lower incidence of HF (o-7\%) was found in other studies in patients treated with $4-18 \mathrm{~g} / \mathrm{m}^{2} /$ cycle of ifosfamide and prior or concurrent anthracyclines [47-5I]. Ifosfamide therapy without co-administration of anthracyclines is assumed to be associated with a low risk of CTRCD $(<\mathrm{I} \%)$, as was reported in a review of 1508 patients receiving ifosfamide monotherapy [52] and a randomized trial comparing treatment with doxorubicin to etoposide and ifosfamide $\left(12 \mathrm{~g} / \mathrm{m}^{2}\right.$ per cycle) [53]. Other cardiac side effects of ifosfamide such as arrhythmias are mainly reversible after discontinuation of the drug [52,54].

\section{Cyclophosphamide}

Cyclophosphamide is an alkylating agent, which is used for a variety of malignancies, including breast cancer, lung cancer, lymphomas, and in conditioning regimens prior to stem cell transplantation. High dose cyclophosphamide $\left(>\mathrm{I} .5 \mathrm{~g} / \mathrm{m}^{2} /\right.$ day) is considered to be highly cardiotoxic, with CTRCD incidences ranging from 7-28\% (Table I) [I,4,6,7]. A single study from Goldberg, et al. detected HF in I4 out of 80 anthracycline-naïve patients after treatment with cyclophosphamide prior to bone marrow transplantation [55]. HF occurred within Io days after receiving the first dose of cyclophosphamide, and was fatal in 6 of I4 patients. A high daily dose $\left(>\mathrm{I} .55 \mathrm{~g} / \mathrm{m}^{2}\right)$ resulted in greater incident of $\mathrm{HF}(\mathrm{I} 3 / 52 ; 25 \%)$, compared to daily doses $<\mathrm{I} .55 \mathrm{~g} / \mathrm{m}^{2}(\mathrm{I} / 32 ; 3 \%)$. Six studies, in which most of the patients had prior treatment with anthracyclines, reported a HF incidence that ranged from $2.3-30.2 \%$, after treatment with high dose cyclophosphamide [56-6I]. Cardiotoxic effects, described as HF and myopericarditis, mostly developed within 2 weeks after administration of cyclophosphamide and recovered within days to weeks. However, as noted above, this was fatal in some cases, with endothelial damage, myopericarditis and diffuse intramyocardial hemorrhage on postmortem histopathological examination [57]. These histopathological findings differ from those seen in anthracycline-induced cardiotoxicity, which may reflect different mechanisms of cardiotoxicity with this agent. 


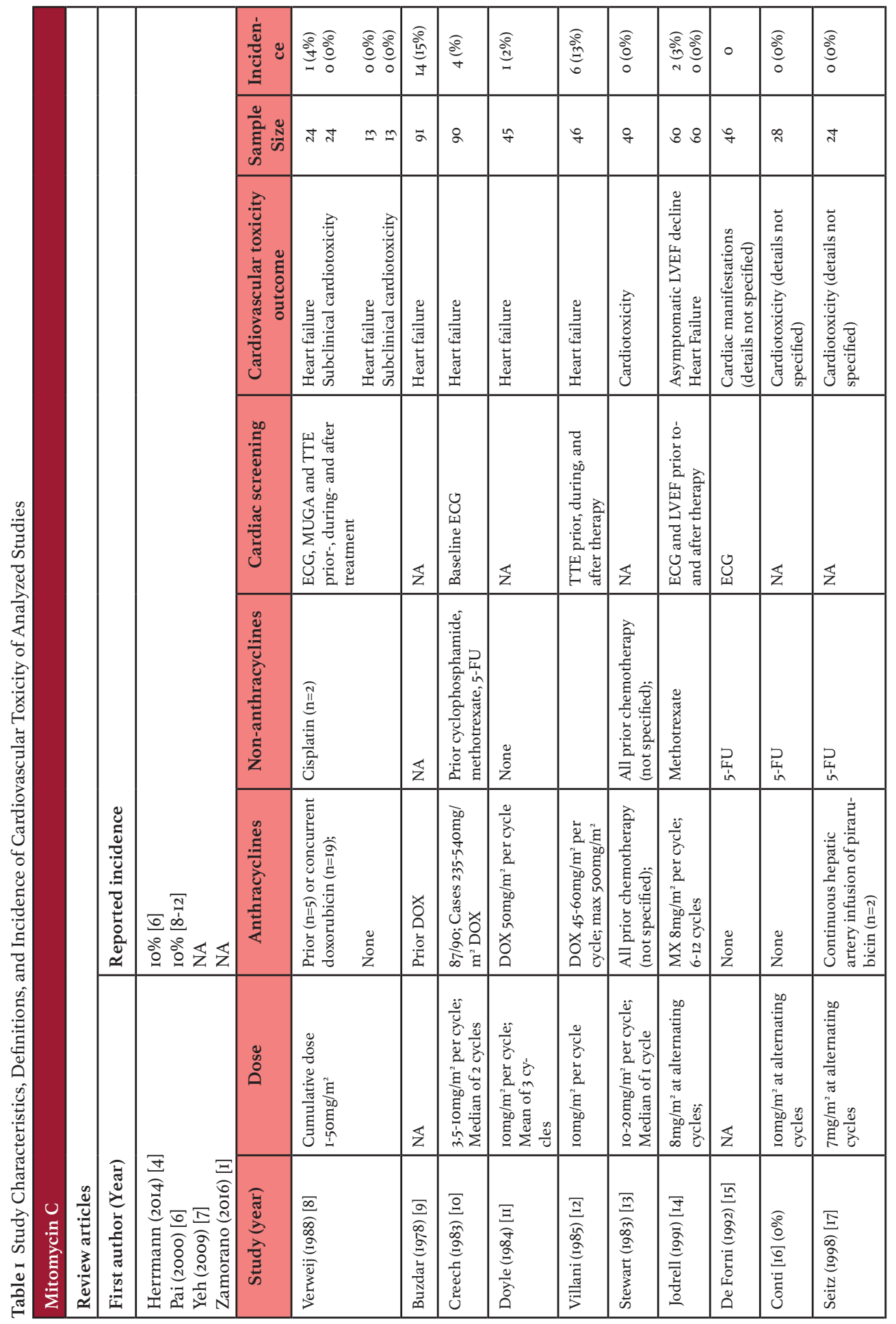



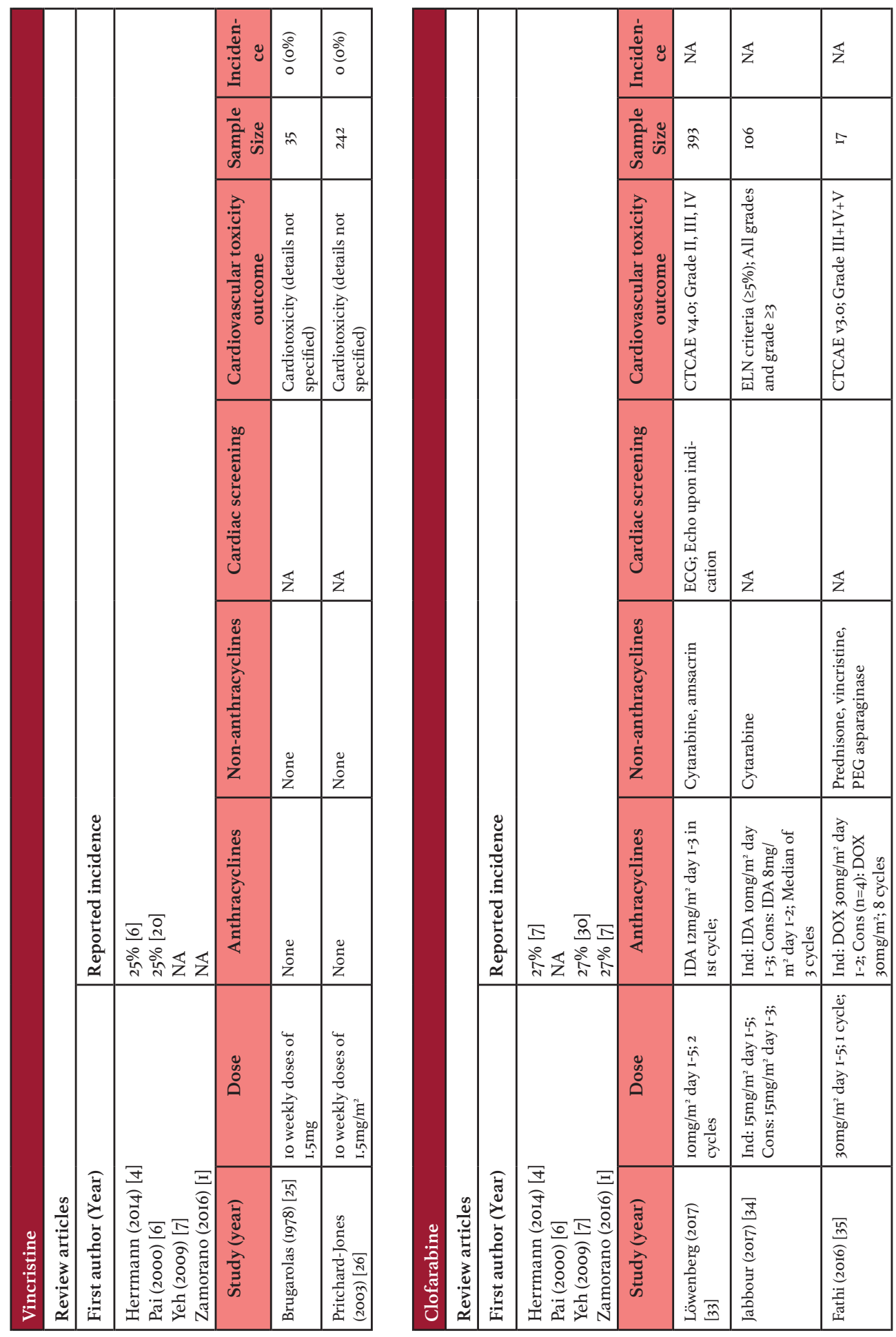


\begin{tabular}{|c|c|c|c|c|c|c|c|c|c|c|}
\hline "艹 & 产 & $\mathbb{z}$ & $\mathbb{z}$ & 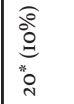 & 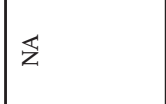 & $\begin{array}{l}\bar{\circ} \\
\stackrel{\circ}{\circ}\end{array}$ & $\bar{z} \quad \widehat{a}$ & 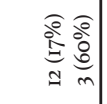 & $\mathbb{z}$ & $\mathbb{z}$ \\
\hline है: & $\stackrel{i s}{i}$ & $\Rightarrow$ & $q$ & 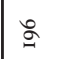 & 8 & ঃ & $\cong \quad \Xi$ & Rin & 8 & 7 \\
\hline 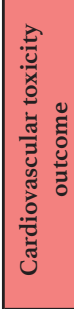 & 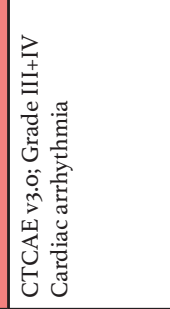 & 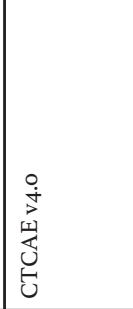 & 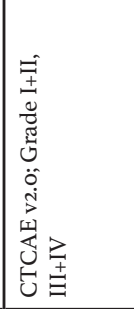 & 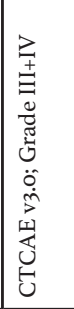 & 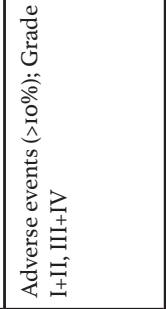 & 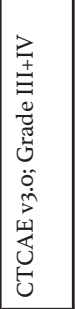 & 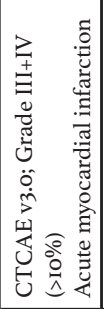 & 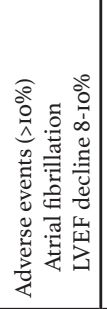 & 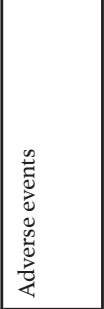 & 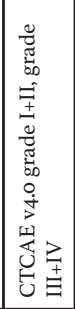 \\
\hline है & $\frac{\pi}{z}$ & $\mathbb{z}$ & $\mathbb{z}$ & $\mathbb{z}$ & 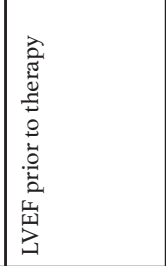 & $\mathbb{z}$ & 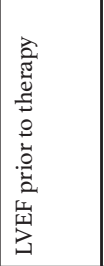 & 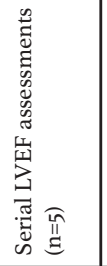 & 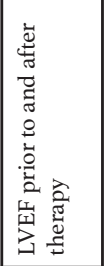 & $\overleftrightarrow{z}$ \\
\hline 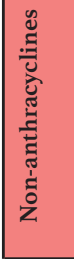 & 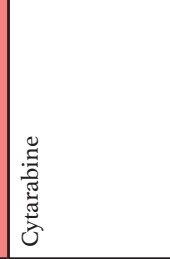 & 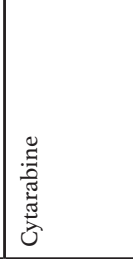 & 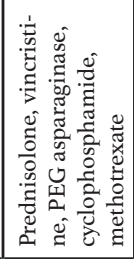 & : & 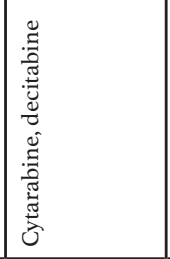 & 产 & 总 & 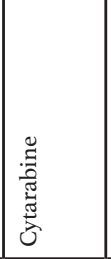 & 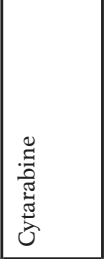 & 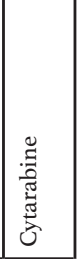 \\
\hline 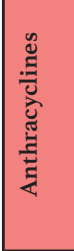 & 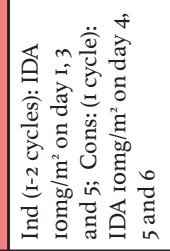 & $\begin{array}{l}\text { : } \\
\text { ż }\end{array}$ & 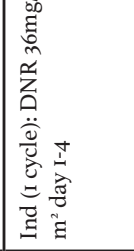 & $\begin{array}{l}\text { 号 } \\
\text { ż }\end{array}$ & 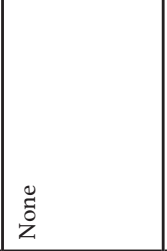 & 产 & 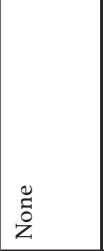 & 荾 & 䓂 & 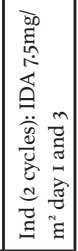 \\
\hline 足 & 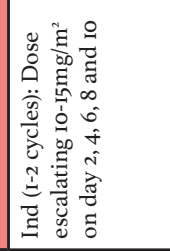 & 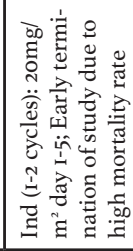 & 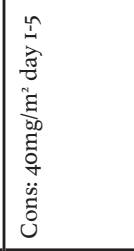 & 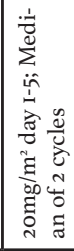 & 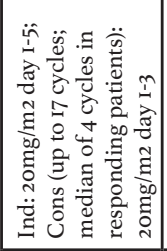 & 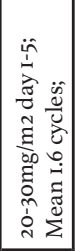 & 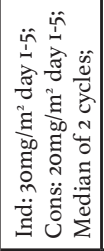 & 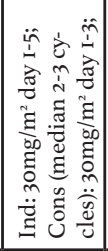 & 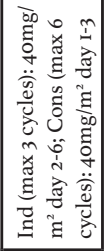 & 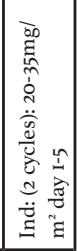 \\
\hline 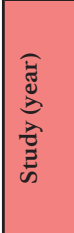 & 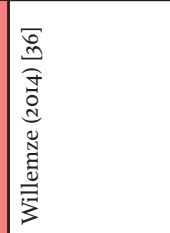 & 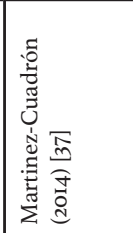 & 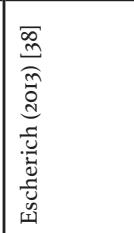 & 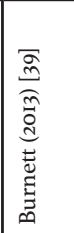 & 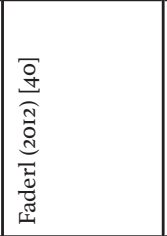 & 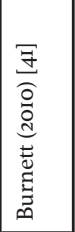 & 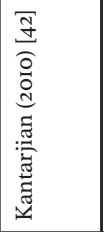 & 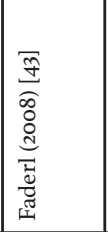 & 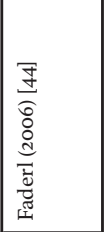 & 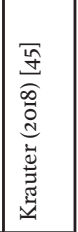 \\
\hline
\end{tabular}




\begin{tabular}{|c|c|c|c|c|c|c|c|c|c|}
\hline & & & 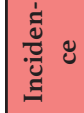 & 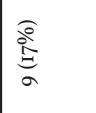 & o̊ & बे & 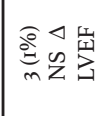 & $\frac{\substack{0 \\
\text { a }}}{\mathrm{a}}$ & oे \\
\hline & & & $\begin{array}{l}\text { है } \\
\text { हू } \\
\text { लू }\end{array}$ & $\approx$ & $\stackrel{2}{:}$ & tr & ్ㅏㅁ & જે & $\stackrel{\infty}{\exists}$ \\
\hline & & & 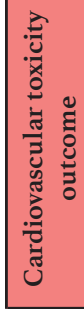 & 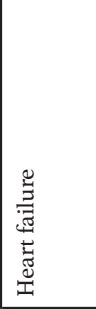 & 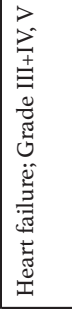 & 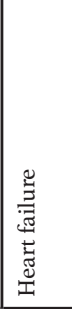 & 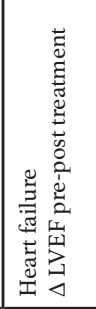 & 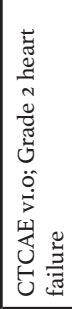 & 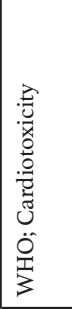 \\
\hline & & & 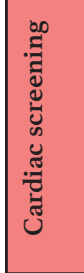 & $\overleftrightarrow{z}$ & $\overleftrightarrow{z}$ & $\overleftrightarrow{\text { 乙 }}$ & 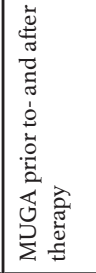 & 艺 & $\overleftrightarrow{z}$ \\
\hline & & & 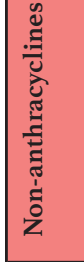 & 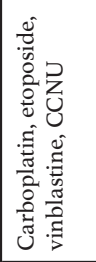 & 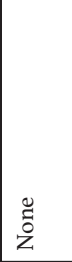 & 气̆ & 气̆ & 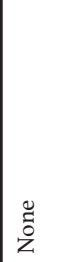 & 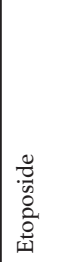 \\
\hline & 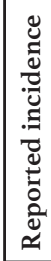 & 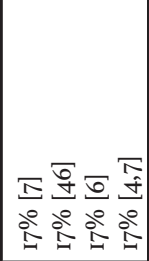 & 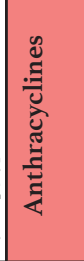 & 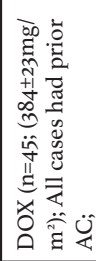 & 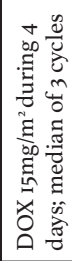 & 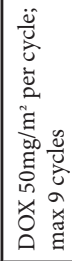 & 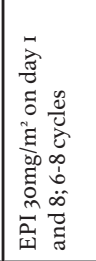 & 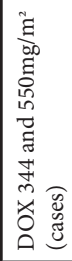 & $\begin{array}{l} \\
\vdots \\
\check{0} \\
z\end{array}$ \\
\hline & & & $\begin{array}{l}\dot{0} \\
\dot{8} \\
0\end{array}$ & 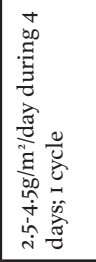 & 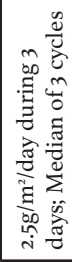 & 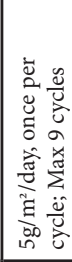 & 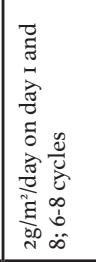 & 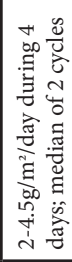 & 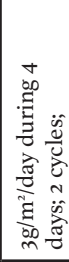 \\
\hline 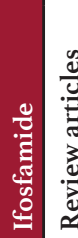 & 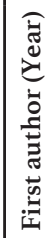 & 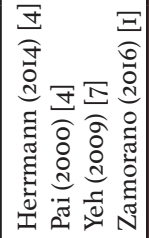 & $\begin{array}{l}\text { है } \\
\text { है } \\
\text { है } \\
\text { है } \\
\text { के }\end{array}$ & 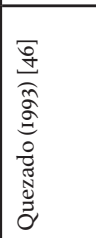 & 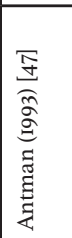 & 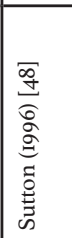 & 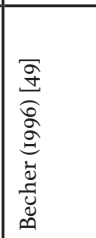 & 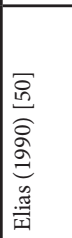 & 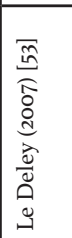 \\
\hline
\end{tabular}




\begin{tabular}{|c|c|c|c|c|c|c|c|c|c|c|}
\hline & & & : & 高 & 高 & 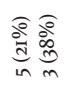 & $\begin{array}{l}\text { वे } \\
\text { da } \\
\frac{d}{d}\end{array}$ & 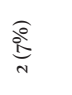 & 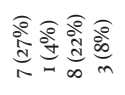 & $\begin{array}{l}\text { 高高高 } \\
\text { in }\end{array}$ \\
\hline & & & 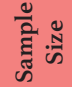 & $\infty$ & 寸 & $4 \infty$ & $\cong$ & ì & ర్N & q $q$ \\
\hline & & & 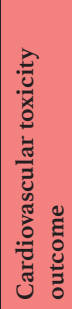 & 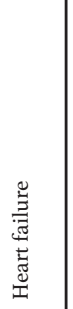 & 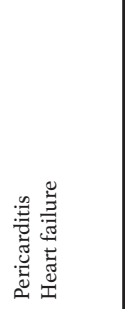 & 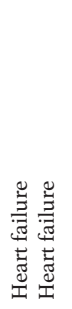 & 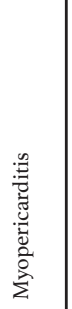 & 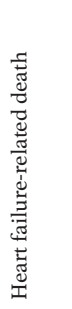 & 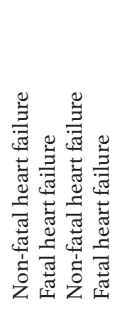 & 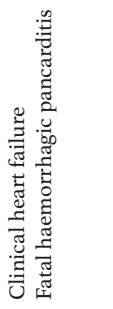 \\
\hline & & & 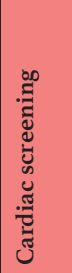 & 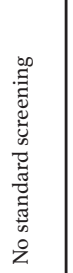 & 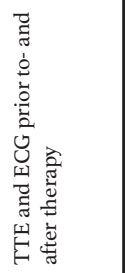 & $\stackrel{\pi}{\sqrt{5}}$ & ż & $\mathbb{z}$ & 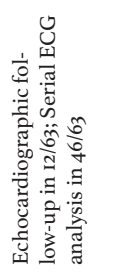 & 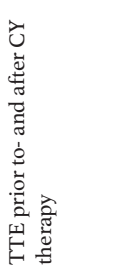 \\
\hline & & & 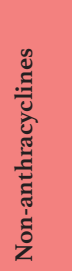 & $\begin{array}{l}0 \\
\text { z̆ }\end{array}$ & 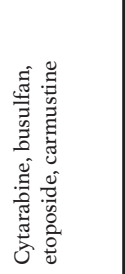 & 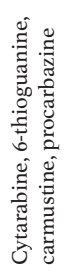 & 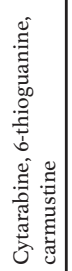 & 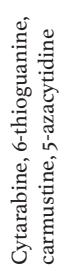 & 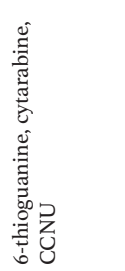 & 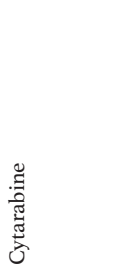 \\
\hline & 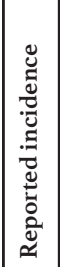 & 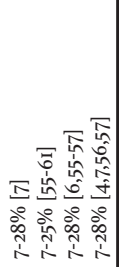 & 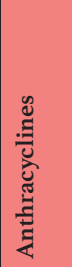 & 气̊̆ & 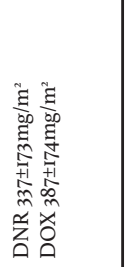 & 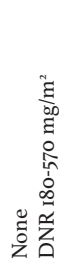 & 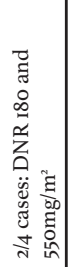 & 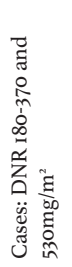 & 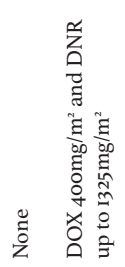 & 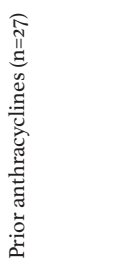 \\
\hline & & & $\begin{array}{l}\mathscr{D} \\
\stackrel{0}{\circ}\end{array}$ & 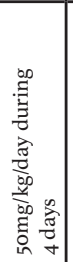 & 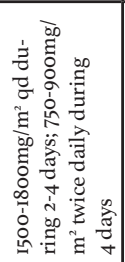 & 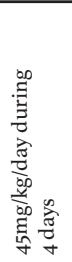 & 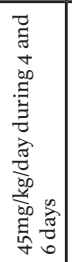 & 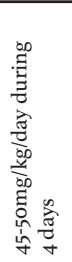 & 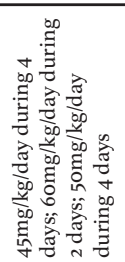 & 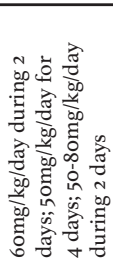 \\
\hline 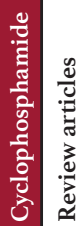 & 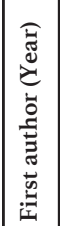 & 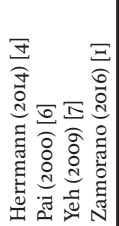 & 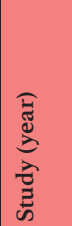 & 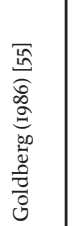 & 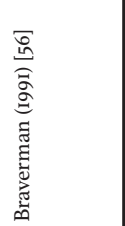 & 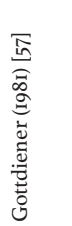 & 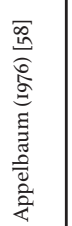 & 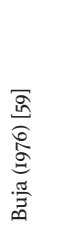 & 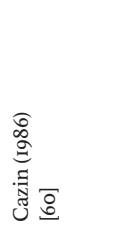 & 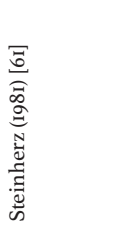 \\
\hline
\end{tabular}




\section{Discussion}

The field of cardio-oncology has made substantial progress in recent years. Nevertheless, there is still a gap in knowledge concerning the cardiotoxic profiles of systemic, non-anthracycline anticancer agents.

Firstly, we observed that CTRCD incidence rates were based on studies that administered these agents as part of combination therapy. Patients typically received prior or concurrent anthracyclines, which makes it difficult to distinguish the true cardiotoxic effect of these chemotherapeutics. Especially for MMC and ifosfamide, the reported high cardiotoxicity rates can likely be attributed to the cardiotoxic effects of anthracyclines.

Secondly, the 4 review articles report the incidence of CTRCD, which definition covers both asymptomatic and symptomatic heart failure. The studies from which these numbers originate, mainly report on the incidence of clinical heart failure. In a majority of cases these studies do not mention whether active screening of cardiac function was performed. Insufficient monitoring may have led to an underreporting of an asymptomatic decline in left ventricular function. Another limitation in the field is that reviews report incidence rates from studies performed many decades ago (Central Illustration). Back then, the diagnosis of CTRCD was predominantly based on signs of clinical heart failure. More recently, new imaging techniques and biomarkers are used to detect CTRCD in an earlier stage, before the occurrence of clinical heart failure. Meanwhile, multiple clinical trials within the field of oncology have been performed, providing systematic reports of adverse events. A systematic analysis of the cardiovascular toxicity reported in these trials is likely to provide a more accurate and precise estimate of cardiotoxicity rates of systemic anticancer agents, as compared to the incidence rates from small, older studies. Recently, a systematic review and meta-analysis on carfilzomib-associated cardiovascular adverse events was published, which provides an excellent example of how the cardiotoxic profile of agents can be analyzed in a comprehensive manner [62].

A majority of clinical trials within the field of oncology report on cardiovascular side effects, without differentiating between arrhythmias, pericardial disease, ischemic heart disease and myocardial dysfunction. Reporting overall cardiotoxicity does not provide information on the underlying pathologic mechanism, which is important since the different side effects need different approaches to screening, prevention and treatment. Therefore, it would be important for future clinical trials to provide a more detailed description of cardiovascular adverse effects instead of overall 'cardiotoxicity.' Also, reporting whether cardiovascular screening was systematically performed aids in the interpretation of reported side effects, since not all adverse effects are overt and may not be evident if screening is not performed. Rigorous reporting standards are likely to advance our understanding of the cardiotoxicity of cancer therapeutics.

\section{Limitations}

The current study gives insight in the scientific evidence on which the currently used CTRCD incidence rates for these five non-anthracycline agents have been based. We believe this study is illustrative of the pitfalls when interpreting cardiotoxicity data. However, for 
all agents except clofarabine, we did not perform a systematic literature search. In the absence of this, the cardiotoxicity of these chemotherapeutics remains uncertain. Another limitation of this study is that we focused on CTRCD while there are many other cardiovascular side effects of anticancer treatment including arrhythmias and myocarditis. However, we do believe that the findings of this study stress the importance of critically re-evaluating the cardiotoxicity profile of the chemotherapeutics addressed, by performing comprehensive literature reviews and meta-analyses. These analyses should not only focus on CTRCD, but also evaluate the risk of other types of cardiotoxicity and patient characteristics influencing the susceptibility of developing these treatment-related side effects.

\section{Conclusion}

Our literature search of 5 non-anthracycline anticancer agents which previously have been recognized to be highly cardiotoxic in landmark review articles, revealed that the reported CTRCD incidence rates for MMC, vincristine, ifosfamide and clofarabine are based on studies in which many patients received prior or concurrent anthracyclines. This complicates the interpretation of their role in causing CTRCD. We have only found convincing evidence of cardiotoxicity for high dose cyclophosphamide. Based on our findings, we advise clinicians to take the reference background into account when using the currently reported incidence rates for CTRCD risk stratification. For future studies within the field, we advise that the cardiotoxicity profile of individual agents, and also of antineoplastic regimens as a whole, are needed, particularly when multiple, potentially cardiotoxic agents are combined. Future clinical trials need to provide a more detailed description of cardiovascular side effects instead of overall 'cardiotoxicity.' Furthermore, international registries need to be developed to collect real-world observational data outside the context of randomized controlled trials. 


\section{References}

I Zamorano J, Lancellotti P, Rodriguez Munoz D, et al. 20I6 ESC Position Paper on cancer treatments and cardiovascular toxicity developed under the auspices of the ESC Committee for Practice Guidelines. Eur Heart J 20I6;37:2768-280I.

2 Swain SM, Whaley FS, Ewer MS. Congestive heart failure in patients treated with doxorubicin: A retrospective analysis of three trials. Cancer 2003;97:2869-2879.

3 Fornaro A, Olivotto I, Rigacci L, et al. Comparison of long-term outcome in anthracycline-related versus idiopathic dilated cardiomyopathy: a single centre experience. Eur J Heart Fail 20I8;20:898-906.

4 Herrmann J, Lerman A, Sandhu N, Villarraga H, Mulvagh S, Kohli M. Evaluation and management of patients with heart disease and cancer: Cardio-oncology. Mayo Clin Proc 2014;89:I287-I306.

5 Cardinale D, Colombo A, Lamantia G, et al. Anthracycline-Induced Cardiomyopathy Clinical Relevance and Response to Pharmacologic Therapy. J Am Coll Cardiol 20I0;55:213-220.

6 Pai V, Nahata M. Cardiotoxicity of chemotherapeutic agents. Incidence, treatment and prevention. Drug Saf 2000;22:263-302.

7 Yeh E, Bickford C. Cardiovascular complications of cancer therapy: Incidence, pathogenesis, diagnosis, and management. J Am Coll Cardiol 2009;53:223I-2247.

8 Verweij J, Funke-Kupper A, Teule G, Pinedo H. A prospective study on the dose dependency of cardiotoxicity induced by mitomycin C. Med Oncol Tumor Pharmacother 1988;5:159-163.

9 Buzdar A, Legha S, Tashima C, et al. Adriamycin and mitomycin C: possible synergistic cardiotoxicity. Cancer Treat Rep 1978;62:1005-I008.

Io Creech R, Catalano R, Shah M, Dayal H. An Effective Low-Dose Mitomycin Regimen for Hormonaland Chemotherapy-Refractory Patients with Metastatic Breast Cancer. Cancer 1983;51:I034-I040.

II Doyle L, Ihde D, Carney D, et al. Combination chemotherapy with doxorubicin and mitomycin C in non-small cell bronchogenic carcinoma. Severe pulmonary toxicity from 3 weekly mitomycin. Am J Clin Oncol 1984;7:719-724.

I2 Villani F, Comazzi R, Lacaita G, et al. Possible enhancement of the cardiotoxicity of doxorubicin when combined with mitomycin C. Med Oncol Tumor Pharmacother 1985;2:93-94.

I3 Stewart D, Maroun J, Young V, et al. Feasibility study of combining metronidazole with chemotherapy. J Clin Oncol 1983;:1:I7-23.

I4 Jodrell D, Smith I, Mansi J, et al. A randomised comparative trial of mitozantrone/methotrexate/ mitomycin $\mathrm{C}(\mathrm{MMM})$ and cyclophosphamide/methotrexate/5 $\mathrm{FU}(\mathrm{CMF})$ in the treatment of advanced breast cancer. Br J Cancer England 1991;63:794-798.

I5 Forni M De, Malet-Martino P, Jaillais P, et al. Cardiotoxicity of high-dose continuous infusion fluorouracil: A prospective clinical study. J Clin Oncol I992;IO:I795-I80I.

I6 Conti J, Kemeny N, Saltz L, Andre A, Grossano D, Bertino J. Continuous infusion fluorouracil/ leucovorin and bolus mitomycin-C as a salvage regimen for patients with advanced colorectal cancer. Cancer 1995;75:769-774.

I7 Seitz J, Perrier H, Giovannini M., Capodano G, Bernardini D, Bardou V-J. 5-Fluorouracil, High-Dose Folinic Acid and Mitomycin C Combination Chemotherapy in Previously Treated Patients with Advanced Colorectal Carcinoma. J Chemother 1998;I0:258-265.

I8 Roca E, Bruera E, Politi P, et al. Vinca alkaloid-induced cardiovascular autonomic neuropathy. Cancer Treat Rep 1985;69:I49-I5I.

19 Hirvonen H, Salmi T, Heinonen E, Antila K, Välimäki I. Vincristine treatment of acute lymphoblastic leukemia induces transient autonomic cardioneuropathy. Cancer 1989;64:80I-805.

20 Roberts W, Glancy D, Devita V. Heart in malignant lymphoma (Hodgkin's disease, lymphosarcoma, reticulum cell sarcoma and mycosis fungoides). A study of 196 autopsy cases. Am J Cardiol 1968;22:85I07.

2I Gros R, Hugon V, Thouret J, Peigne V. Coronary Spasm after an Injection of Vincristine. Chemotherapy 20I7;62:I69-I7I.

22 Cargill R, Boyter A, Lipworth B. Reversible myocardial ischaemia following vincristine containing chemotherapy. Respir Med 1994;88:709-7IO.

23 Mandel E, Lewinski U, Djadetti M. Vincristine-induced myocardial infarction. Cancer 1975;36:19791982. 
24 Somers G, Abramov M, Witter M, Naets J. Myocardial infarction: a complication of vincristine treatment? Lancet 1976;2:690.

25 Brugarolas A, Lacave A, Ribas A, Garcia Miralles M. Vincristine (NSC 67574) in non-small cell bronchogenic carcinoma. Results of a phase II clinical study. Eur J Cancer 1978;14:50I-505.

26 Pritchard-Jones K, Kelsey A, Vujanic G, Imeson J, Hutton C, Mitchell C. Older age is an adverse prognostic factor in stage I, favorable histology Wilms' tumor treated with vincristine monochemotherapy: A study by the United Kingdom Children's Cancer Study Group, Wilm's Tumor Working Group. J Clin Oncol 2003;21:3269-3275.

27 Chatterjee K, Zhang J, Honbo N, Simonis U, Shaw R, Karliner J. Acute vincristine pretreatment protects adult mouse cardiac myocytes from oxidative stress. J Mol Cell Cardiol 2007;43:327-336.

28 Chatterjee K, Zhang J, Tao R, Honbo N, Karliner J. Vincristine attenuates doxorubicin cardiotoxicity. Biochem Biophys Res Commun 2008;373:555-560.

29 Zhenchuk A, Lotfi K, Juliusson G, Albertioni F. Mechanisms of anti-cancer action and pharmacology of clofarabine. Biochem Pharmacol 2009; 78(II):I35I-9.

$30 \mathrm{https} / /$ www.accessdata.fda.gov/drugsatfda_docs/label/2004/02I673lbl.pdf. Accessed on 07/o8/2018.

3I Jeha S, Gaynon P, Razzouk B, et al. Phase II Study of Clofarabine in Pediatric Patients With Refractory or Relapsed Acute Lymphoblastic Leukemia. J Clin Oncol 2006;24:I917-I923.

32 Jeha S, Razzouk B, Rytting M, et al. Phase II study of clofarabine in pediatric patients with refractory or relapsed acute myeloid leukemia. J Clin Oncol 2009;27:4392-4397.

33 Löwenberg B, Pabst T, Maertens J, et al. Therapeutic value of clofarabine in younger and middle-aged (I8-65 years) adults with newly diagnosed AML. Blood 2017;I29(I2):I636-I645.

34 Jabbour E, Short NJ, Ravandi F, et al. A Randomized Phase 2 Study of Idarubicin and Cytarabine With Clofarabine or Fludarabine in Patients With Newly Diagnosed Acute Myeloid Leukemia Cancer 2017;123:4430-9.

35 Fathi AT, DeAngelo DJ, Stevenson KE, et al. Phase II study of intensified chemotherapy and allogeneic hematopoietic stem cell transplantation for older patients with acute lymphoblastic leukemia (ALL). Cancer 2016;122:2379-2388

36 Willemze R, Suciu S, Muus P, et al. Clofarabine in combination with a standard remission induction regimen (cytosine arabinoside and idarubicin) in patients with previously untreated intermediate and bad-risk acute myelogenous leukemia (AML) or high-risk myelodysplastic syndrome (HR-MDS): phase I results of an ongoing phase I/II study of the leukemia groups of EORTC and GIMEMA (EORTC GIMEMA 0606I/AML-I4A trial). Ann Hematol 20I4; 93:965-975.

37 Martínez-Cuadrón D, Montesinos P, Oriol A, et al. Phase II trials to assess the safety and efficacy of clofarabine in combination with low-dose cytarabine in elderly patients with acute myeloid leukemia. Ann Hematol 20I4; 93:43-46.

38 Escherich G, zur Stadt U, Zimmermann M, et al. Clofarabine in combination with pegylated asparaginase in the frontline treatment of childhood acute lymphoblastic leukemia: a feasibility report from the CoALL 08-09 trial. Br J Haematol 2013; I63(2): 240-7.

39 Burnett $\mathrm{AK}$, Russell $\mathrm{NH}$, Hunter $\mathrm{AE}$, et al. Clofarabine doubles the response rate in older patients with acute myeloid leukemia but does not improve survival. Blood 2013;I22(8):1384-94.

40 Faderl S, Ravandi F, Huang X, et al. Clofarabine plus low-dose cytarabine followed by clofarabine plus low-dose cytarabine alternating with decitabine in acute myeloid leukemia frontline therapy for older patients. Cancer 20I2;II8(I8):447I-7.

4I Burnett AK, Russell NH, Kell J, et al. European development of clofarabine as treatment for older patients with acute myeloid leukemia considered unsuitable for intensive chemotherapy. J Clin Oncol 20I0;28(I4):2389-95

42 Kantarjian HM, Claxton D, et al. Phase II study of clofarabine monotherapy in previously untreated older adults with acute myeloid leukemia and unfavorable prognostic factors. J Clin Oncol 20IO;28(4):549-55.

43 Faderl S, Ravandi F, Huang X, et al. A randomized study of clofarabine versus clofarabine plus lowdose cytarabine as front-line therapy for patients aged 6o years and older with acute myeloid leukemia and high-risk myelodysplastic syndrome. Blood 2008;II2(5):1638-45. 
44 Faderl S, Verstovsek S, Cortes J, et al. Clofarabine and cytarabine combination as induction therapy for acute myeloid leukemia (AML) in patients 50 years of age or older. Blood 2006;IO8(I):45-5I.

45 Krauter J, Fiedler W, Schlenk FR, et al. Phase I/II study on cytarabine and idarubicin combined with escalating doses of clofarabine in newly diagnosed patients with acute myeloid leukaemia and high risk for induction failure (AMLSG I7-IO CIARA trial). Br J Haematol 2018.183(2):235-24I

46 Quezado Z, Wilson W, Cunnion R, et al. High-dose ifosfamide is associated with severe, reversible cardiac dysfunction. Ann Intern Med 1993;II8:3I-36.

47 Antman K, Crowley J, Balcerzak S, et al.An intergroup phase III randomized study of doxorubicin and dacarbazine with or without ifosfamide and mesna in advanced soft tissue and bone sarcomas. J Clin Oncol I993;II:I276-I285.

48 Sutton G, Blessing J, Malfetano J. Ifosfamide and doxorubicin in the treatment of advanced leiomyosarcomas of the uterus: A Gynecologic Oncology Group study. Gynecol Oncol 1996;62:226-229.

49 Becher R, Kloke O, Hayungs J, et al. Epirubicin and ifosfamide in metastatic breast cancer. Semin Oncol 1996;23:28-33.

50 Elias A, Eder J, Shea T, Begg C, Frei E, Antman K. High-dose ifosfamide with mesna uroprotection: A phase I study. J Clin Oncol 1990;8:I70-I78.

5I Brade WP, Herdrich K, Varini M. Ifosfamide-pharmacology, safety and therapeutic potential. Cancer Treat Rev I985;I2:I-47.

52 Brade W, Herdrich K, Kachel-Fischer U, Araujo C. Dosing and side-effects of ifosfamide plus mesna. J Cancer Res Clin Oncol I99I;II7:I64-I86.

53 Le Deley M, Guinebretière J, Gentet J, et al. SFOP OS94: A randomised trial comparing preoperative high-dose methotrexate plus doxorubicin to high-dose methotrexate plus etoposide and ifosfamide in osteosarcoma patients. Eur J Cancer 2007;43:752-76I.

54 Kandylis K, Vassilomanolakis M, Tsoussis S, Efremidis A. Ifosfamide cardiotoxicity in humans. Cancer Chemother Pharmacol 1989;24:395-396.

55 Goldberg M, Antin J, Guinan E, Rappeport J. Cyclophosphamide cardiotoxicity: an analysis of dosing as a risk factor. Blood I986;68:III4-III8.

56 Braverman A, Antin J, Plappert M, Cook E, Lee R. Cyclophosphamide cardiotoxicity in bone marrow transplantation: A prospective evaluation of new dosing regimens. J Clin Oncol 1991;9:1215-1223.

57 Gottdiener J, Appelbaum F, Ferrans V, Deisseroth A, Ziegler J. Cardiotoxicity Associated with HighDose Cyclophosphamide Therapy. Arch Intern Med I981;141:758-763.

58 Appelbaum F, Strauchen J, Graw R, et al. Acute lethal carditis caused by high-dose combination chemotherapy: a unique clinical and pathological entity. Lancet 1976;307:58-62.

59 Buja L, Ferrans V, Grow R. Cardiac pathologic findings in patients treated with bone marrow transplantation. Hum Pathol 1976;7:17-45.

60 Cazin B, Gorin N, Laporte J, et al. Cardiac Complications After Bone Marrow Transplantation. A report on a series of 63 consecutive transplantations. Cancer 1986;57:206I-2069.

6I Steinherz L, Steinherz P, Mangiacasale D, et al. Cardiac changes with cyclophosphamide. Med Pediatr Oncol 198I;9:4I7-422.

62 Waxman AJ, Clasen S, Hwang WT et al. Carfilzomib-Associated Cardiovascular Adverse Events: A systematic Review and Meta-analysis. JAMA Oncol 2018;4(3):er74519; 


\section{Chapter 4}

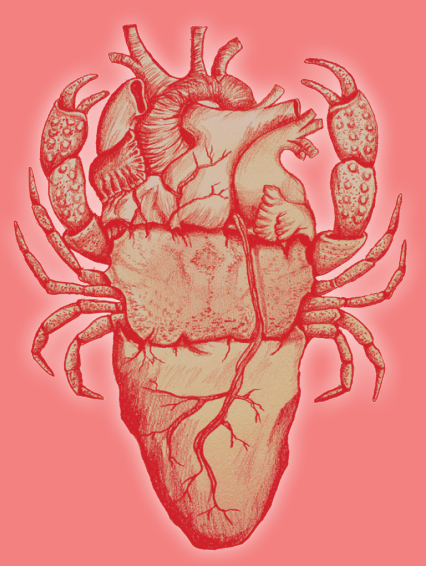




\section{Cardiovascular adverse events in patients with} non-Hodgkin lymphoma treated with first-line cyclophosphamide, doxorubicin, vincristine, and prednisone (CHOP) or CHOP with rituximab (R-CHOP)

a systematic review and meta-analysis

Lancet Haematology 2020; 7(4):e295-e308

JAM Kamphuis ${ }^{1^{*}}$, M Linschoten $^{1^{*}}$, A van Rhenen ${ }^{2}$,

LP Bosman' ${ }^{1}$, MJ Cramer ${ }^{1}$, PA Doevendans ${ }^{1,3,4}$,

AJ Teske ${ }^{\mathrm{I} *}, \mathrm{FW}$ Asselbergs ${ }^{\mathrm{I}, 3,5 *}$

\footnotetext{
* Joint first authors
}

\# Joint last authors

I Department of Cardiology, Division of Heart and Lungs, University Medical Centre Utrecht, Utrecht, The Netherlands 2 Department of Haematology, Cancer Centre, University Medical Centre Utrecht, Utrecht, The Netherlands 3 Netherlands Heart Institute, Utrecht, The Netherlands

4 Central Military Hospital, Utrecht, the Netherlands

5 Health Data Research UK, Institute of Health Informatics and Institute of Cardiovascular Science, Faculty of Population Health Sciences, University College London, London, UK 


\section{Summary}

Background Patients treated for non-Hodgkin lymphoma are at risk of cardiovascular adverse events, with the risk of heart failure being particularly high. A regimen of cyclophosphamide, doxorubicin, vincristine, and prednisone, with (R-CHOP) or without (CHOP) rituximab is the standard first-line treatment for aggressive non-Hodgkin lymphoma, and doxorubicin and cyclophosphamide are both associated with left ventricular dysfunction. The aim of this systematic review and meta-analysis was to evaluate the cardiovascular toxicity of this regimen.

Methods We systematically searched PubMed, EMBASE, and the Cochrane Library from database inception to June 3, 20I9, for clinical trials and observational studies in adult patients with non-Hodgkin lymphoma (diffuse large B-cell lymphoma, follicular lymphoma, mantle cell lymphoma, peripheral T-cell lymphoma, and non-Hodgkin lymphoma not otherwise specified) that received first-line treatment with R-CHOP or CHOP. Studies reporting on cardiovascular adverse events and treatment-related cardiovascular mortality were included. Abstracts and articles not written in English were excluded. The main outcomes were the proportion of patients with grade 3-4 cardiovascular adverse events and heart failure. Meta-analyses of one-sample proportions were done in all patients receiving $\mathrm{CHOP}$ or R-CHOP. Subgroup analyses on summary estimates were done to determine the effect of number of CHOP or R-CHOP cycles, cycle interval, age, and sex.

Findings Of $23 \mathrm{I} 4$ identified entries, 137 studies (2I 2II patients) published between April, I984, and June, 2019 were eligible (954I patients treated with CHOP, II 293 patients treated with R-CHOP, 377 both regimens used in the study; median follow-up $39 \cdot 0$ months [IQR 25·5-52·8]). From the included studies, 85 subgroups were treated with $\mathrm{CHOP}, 76$ with $\mathrm{R}-\mathrm{CHOP}$, and in four studies both CHOP and R-CHOP were used without a subdivision in separate groups. The pooled proportion for grade 3-4 cardiovascular adverse events, based on 77 studies ( $\mathrm{n}=\mathrm{I} 4$ 35I patients), was $2 \cdot 35 \%$ (95\% CI I·8I-2.93; heterogeneity test $\mathrm{Q}=326 \cdot 2 \mathrm{I}$; $\left.\tau^{2}=0 \cdot 0042 ; \mathrm{I}^{2}=7 \mathrm{I} \cdot 40 \% ; \mathrm{p}<0 \cdot 000 \mathrm{I}\right)$. For heart failure, the pooled proportion, based on 38 studies $\left(\mathrm{n}=5936\right.$ patients), was $4 \cdot 62 \%\left(2 \cdot 25-7 \cdot 65 ;\right.$ heterogeneity test $\mathrm{Q}=527 \cdot 33 ; \tau^{2}=0 \cdot 0384$; $\mathrm{I}^{2}=95 \cdot 05 \%$; p $\left.<0 \cdot 000 \mathrm{I}\right)$, with a significant increase in reported heart failure from $\mathrm{I} \cdot 64 \%(95 \%$ CI $0 \cdot 82-2 \cdot 65)$ to $\mathrm{II} \cdot 72 \%(3 \cdot 00-24 \cdot 53)$ when cardiac function was evaluated post-chemotherapy $(\mathrm{p}=\mathrm{0} \cdot 0 \mathrm{I} 7) .53$ (39\%) of $\mathrm{I} 37$ studies were rated as having high risk of bias for incomplete outcome data and 54 (39\%) for selective reporting.

Interpretation The considerable increase of reported heart failures with cardiac monitoring, indicates that this complication often remains undiagnosed in patients with non-Hodgkin lymphoma who received first-line R-CHOP or CHOP. Our findings are of importance to raise awareness of this complication among clinicians treating patients with non-Hodgkin lymphoma and stresses the need for cardiac monitoring during and after chemotherapy. Prompt initiation of treatment for heart failure in the presymptomatic phase can mitigate the progression to more advanced heart failure stages. 


\section{Research in context}

Evidence before this study

Survivors of non-Hodgkin lymphoma are at risk of cardiovascular disease, which is believed to be related to toxic effects of antineoplastic treatment. Cyclophosphamide, doxorubicin, vincristine, and prednisone with or without rituximab (CHOP or R-CHOP) has been the first-choice regimen for the treatment of aggressive non-Hodgkin lymphomas for decades, but the proportion of patients developing cardiovascular adverse events after treatment has not been comprehensively evaluated. Previous work within the field has mainly focused on unravelling the cardiovascular toxicity of individual drugs, which are often not used as monotherapy. We searched PubMed, EMBASE and the Cochrane Library using a broad search string including the words "lymphoma", "CHOP", and "R-CHOP" from inception of each database to June 3, 2019. Among I888 unique hits, we found no meta-analysis assessing the occurrence of cardiovascular adverse events in patients with non-Hodgkin lymphoma receiving CHOP or R-CHOP.
Added value of this study

Our analysis summarises the occurrence of cardiovascular adverse events in patients treated with $\mathrm{CHOP}$ or R-CHOP as a first-line regimen across 137 studies. In contrast to previous work, we focused on the cardiovascular toxicity of a complete treatment regimen, rather than individual agents. Moreover, to our knowledge, this is the first study that specifically evaluates the consequences of serial cardiac assessment on the reported number of patients with heart failure or left ventricular dysfunction following treatment with $\mathrm{CHOP}$ or R-CHOP.

Implications of all the available evidence

Clinicians should be aware that chemotherapy-related cardiac dysfunction is common after treatment with CHOP or R-CHOP and often does not immediately result in symptomatic heart failure. Our findings stress the importance of close multidisciplinary collaboration between (haemato-)oncologists and cardiologists for the early detection of cardiovascular complications in the pre-symptomatic phase, enabling targeted prevention strategies to improve long-term cardiac outcome in survivors of non-Hodgkin lymphomas.

\section{Introduction}

Non-Hodgkin lymphomas comprise a wide variety of neoplasms arising from the lymphoid tissues. With an estimated 509600 new cases in 20I8, non-Hodgkin lymphoma accounts for approximately $3 \%$ of all cancer cases worldwide [I]. During the last four decades, the survival of patients with these malignancies has improved markedly, and currently $72 \%$ of patients survive up to 5 years after the diagnosis [2]. In line with these developments, the management and prevention of treatment-related side effects is becoming increasingly important.

Growing evidence indicates that the risk of cardiovascular disease is considerably raised in patients with non-Hodgkin lymphoma and survivors [3,4]. The reasons behind this observation are believed to be multifactorial, driven by - among others- shared risk factors between cancer and cardiovascular diseases [5] as well as toxic cardiovascular effects of antineoplastic drugs [6]. In particular, the risk of heart failure is substantially elevated, with an adjusted hazard ratio of I.77 (95\% CI I.5O-2.09) [4] and a standardised incidence ratio of $5 \cdot 4(4 \cdot I-6 \cdot 9)$ for patients treated for non-Hodgkin lymphoma compared with the general population [3]. Moreover, compared with patients treated for other malignancies, the risk of heart failure appears especially high in this patient population [4]. It is conceivable that the chemotherapeutic regimens used to treat these neoplasms play an important role herein. 
The regimen composed of cyclophosphamide $750 \mathrm{mg} / \mathrm{m}^{2}$, doxorubicin $50 \mathrm{mg} / \mathrm{m}^{2}$, vincristine $\mathrm{I} \cdot 4 \mathrm{mg} / \mathrm{m}^{2}$ (maximal dose $2 \mathrm{mg}$ ), and prednisone $500 \mathrm{mg}$ (CHOP) was first introduced in 1976, and soon evolved to become the golden standard for the treatment of patients with aggressive non-Hodgkin lymphoma [7,8]. In patients with B-cell non-Hodgkin lymphoma, $\mathrm{CHOP}$ is combined with the anti-CD20 monoclonal antibody rituximab at a dose of $375 \mathrm{mg} / \mathrm{m}^{2}$ (R-CHOP), as the LNH98-5 trial showed overwhelming benefit of this regimen [9,IO]. The $\mathrm{CHOP}$ and $\mathrm{R}-\mathrm{CHOP}$ regimens contain two compounds - the anthracycline doxorubicin and the alkylator cyclophosphamide - associated with a high risk of left ventricular dysfunction [II]. The dose-dependent incidence of heart failure in patients treated with doxorubicin has, with time, resulted in a restriction of the maximum cumulative dose to $450 \mathrm{mg} / \mathrm{m}^{2}$, correlating with an incidence of symptomatic heart failure of approximately 5\% [6]. Nevertheless, as some patients develop severe heart failure at much lower doses [I2] no safe dose seems to exist and individual tolerability appears to be, for a large part, also dependent on patient-related risk factors [13,I4]. The causal link between cyclophosphamide and left ventricular dysfunction has received far less attention than has that of anthracyclines. Cyclophosphamide- induced cardiotoxicity mostly occurs within days of treatment initiation and can lead to heart failure (in $2-38 \%$ of patients) [ $15-20$ ], (myo)pericarditis (in 9-27\% of patients)[15,20,2I], or both.

Despite the combination of these two highly cardiotoxic agents in CHOP and $\mathrm{R}-\mathrm{CHOP}$, the incidence of cardiovascular adverse events in patients treated with this regimen has been poorly established. An important limitation of previous studies is the inclusion of patients that have received different first-line chemotherapeutic regimens with varying doses of cardiotoxic agents [22-24]. Additionally, the larger studies have only captured cases of symptomatic heart failure leading to hospital contact $[23,24]$, thereby potentially underestimating the true incidence of left ventricular dysfunction. Since CHOP is expected to remain the backbone in the treatment of aggressive non-Hodgkin lymphoma in the upcoming years, insight into the incidence of cardiovascular adverse events in patients treated with this specific regimen is of great importance. Furthermore, identification of factors predisposing patients with non- Hodgkin lymphoma to cardiovascular adverse events can aid in individual risk stratification. With this systematic review and meta-analysis, we aimed to assess the proportion of patients with non-Hodgkin lymphoma developing cardiovascular adverse events after receiving CHOP or R-CHOP as a first-line treatment and identify factors modulating this risk.

\section{Methods}

\section{Search strategy and selection criteria}

This systematic review and meta-analysis is reported in accordance with the guidelines of the Preferred Reporting Items for Systematic Reviews and Meta-Analyses [25]. Two independent researchers (ML and JAMK) searched PubMed, EMBASE, and the Cochrane Library (Cochrane Controlled Register of Trials) from inception of each database to June 3, 20I9. The complete search string is provided in Supplemental File I. We selected clinical 
trials and observational studies in adult patients with non-Hodgkin lymphoma (diffuse large B-cell lymphoma, follicular lymphoma, mantle cell lymphoma, peripheral T-cell lymphoma, and non- Hodgkin lymphoma not otherwise specified) that received first-line treatment with CHOP or R-CHOP. Reviews, letters, commentaries, case reports, conference abstracts, preclinical studies, and articles not written in English were excluded. Studies in which CHOP or R-CHOP was combined with other chemotherapeutic or immunotherapeutic compounds were also excluded. In case other chemotherapeutic or immunotherapeutic drugs were administered sequentially, the study was deemed eligible only if reports of $\mathrm{CHOP}$ and $\mathrm{R}-\mathrm{CHOP}$ toxic effects were available before the initiation of other compounds.

Studies were included in the systematic review if at least one of the following variables could be extracted: number of patients with cardiovascular adverse events reported in grades, preferably with the use of a well-established toxicity grading system to quantify the severity of the adverse events (e.g., WHO toxicity grading scale or the Common Terminology Criteria for Adverse Events [CTCAE] of the US National Cancer Institute); number of patients who died because of cardiovascular adverse events stated to be (possibly) treatment-related; and number of patients with clinical heart failure (i.e., symptomatic left ventricular dysfunction) and, if available, subclinical heart failure (i.e., asymptomatic left ventricular dysfunction). In this Article, we use the term overall heart failure as an umbrella term for both clinical and subclinical heart failure. If cardiovascular adverse events were not reported, only studies explicitly mentioning that no such events occurred were included. In addition, only when all causes of deaths were specified, we assumed no treatment-related cardiovascular deaths had occurred. Two researchers (ML and JAMK) independently extracted the data. In case of discrepancies, the study was re-evaluated and a consensus was reached.

\section{Data analysis}

Study characteristics (sample size, length of follow-up, doses of individual chemotherapeutic agents, cycle interval, number of cycles, patient demographics, and outcome data) were extracted from the included studies and tabulated in a database. If articles were based on the same trial, we selected the most recent one with the longest follow-up or the one reporting the most complete toxicity data.

We did meta-analyses of one-sample proportions to estimate the proportion of patients developing grade 3-4 cardiovascular adverse events overall, heart failure, grade I-2 cardiovascular adverse events, treatment discontinuation due to overall heart failure, and cardiovascular death. A double-arcsine transformation was applied on the proportions to establish a normal distribution appropriate for pooling [26]. Weights for each study in the analysis were based on the inverse of their variance. Proportion estimates are presented with $95 \%$ CIs.

We did subgroup analyses for grade 3-4 cardiovascular adverse events and overall heart failure. These subgroups involved categorical variables (treatment [R-CHOP vs $\mathrm{CHOP}$ ] and cardiac screening after chemotherapy [yes vs no]) and continuous variables 
(number of cycles, interval between cycles, age, and sex [measured as the proportion of female participants]). In subgroup analyses, we estimated the pooled proportion $(95 \% \mathrm{CI})$ of patients in each subgroup for grade 3-4 cardiovascular adverse events and overall heart failure. The difference in pooled proportions across subgroups was tested using a fixed effect model. Additionally, we assessed the effect of each variable on risk (relative risk [ $95 \%$ $\mathrm{CI}$ ) for the variables treatment, number of cycles, interval between cycles, and age. Since screening does not add to the risk of developing cardiovascular adverse events, risks were not determined for this variable. To calculate relative risks, continuous variables were dichotomised using arbitrary cutoffs. In the absence of individual patient data, pooled proportions and relative risks were not determined for female versus male sex. Furthermore, meta-regressions were done to evaluate the association between continuous variables (number of cycles, interval between cycles, age, and sex [measured as the proportion of female participants]) and grade 3-4 cardiovascular adverse events and heart failure. Outcomes of each meta-regression are presented as regression coefficients $(95 \% \mathrm{CI}) . \mathrm{P}<\mathrm{O} \cdot 05$ was considered statistically significant.

We used the Cochrane Risk of Bias Tool to assess bias in individual studies [27]. Since almost all studies compared the anti-neoplastic efficacy of CHOP or R-CHOP with that of another chemotherapeutic regimen, we only extracted information on the occurrence of adverse events for the group of patients receiving CHOP or R-CHOP and subsequently pooled all these single groups from different studies. Therefore, after careful consideration, we decided that random sequence generation, allocation concealment and blinding of participants and personnel, and outcome assessment were unlikely to introduce bias. However, incomplete outcome data and selective reporting were viewed as important potential sources of bias and were assessed for all studies. A quality assessment on the reporting of safety data was done using the checklist of the Consolidated Standards of Reporting Trials (CONSORT) extension for harms [28]. As most studies were primarily done to evaluate the efficacy of CHOP or R-CHOP and not to determine the incidence of cardiovascular adverse events, we deemed the risk of publication bias negligible. For this reason, we did not use the traditional publication bias modelling tool.

From all studies found eligible, we extracted the following counts: the number of patients included in the toxicity analysis; the proportion of patients with grade I (mild), 2 (moderate), 3 (severe), and 4 (life-threatening) cardiovascular adverse events; the number of patients with subclinical and clinical heart failure; the number of patients who discontinued treatment because of treatment-related left ventricular dysfunction or heart failure; the number of (possibly) treatment-related cardiovascular deaths (grade 5 toxicity); the number of patients who died at the end of study follow-up (i.e., all-cause mortality). Heterogeneity between studies was estimated using the $\mathrm{Q}$ statistic, $\mathrm{I}^{2}$, and $\tau^{2}$. Heterogeneity was classified as low (25\%), moderate (50\%), and large (75\%) [29]. A fixed-effect model was used to pool the summary proportions when the heterogeneity was less than $50 \%$; otherwise, a random-effects model was used. 
All statistical analyses were done in $\mathrm{R}$ (version 3.5.I), with the metafor package (version 2.I-O)[30]. Statistical analyses were done in collaboration with an independent clinical epidemiologist (LPB).

\section{Results}

The literature search yielded a total of 2314 records, from which we removed 426 duplicates (Figure I). After abstract screening, we excluded 1534 studies that did not fulfil the inclusion criteria. After assessing the full text of 354 articles, we eventually included I37 studies (2I 2II patients) published between April, I984, and June, 20I9 in the systematic review. Of these, 26 studies compared treatment arms with different numbers of cycles, interval between cycles, or dosing of the individual compounds in CHOP or R-CHOP. Thereby, a total of I65 subgroups of treatment were derived from all 137 studies included in the systematic review.

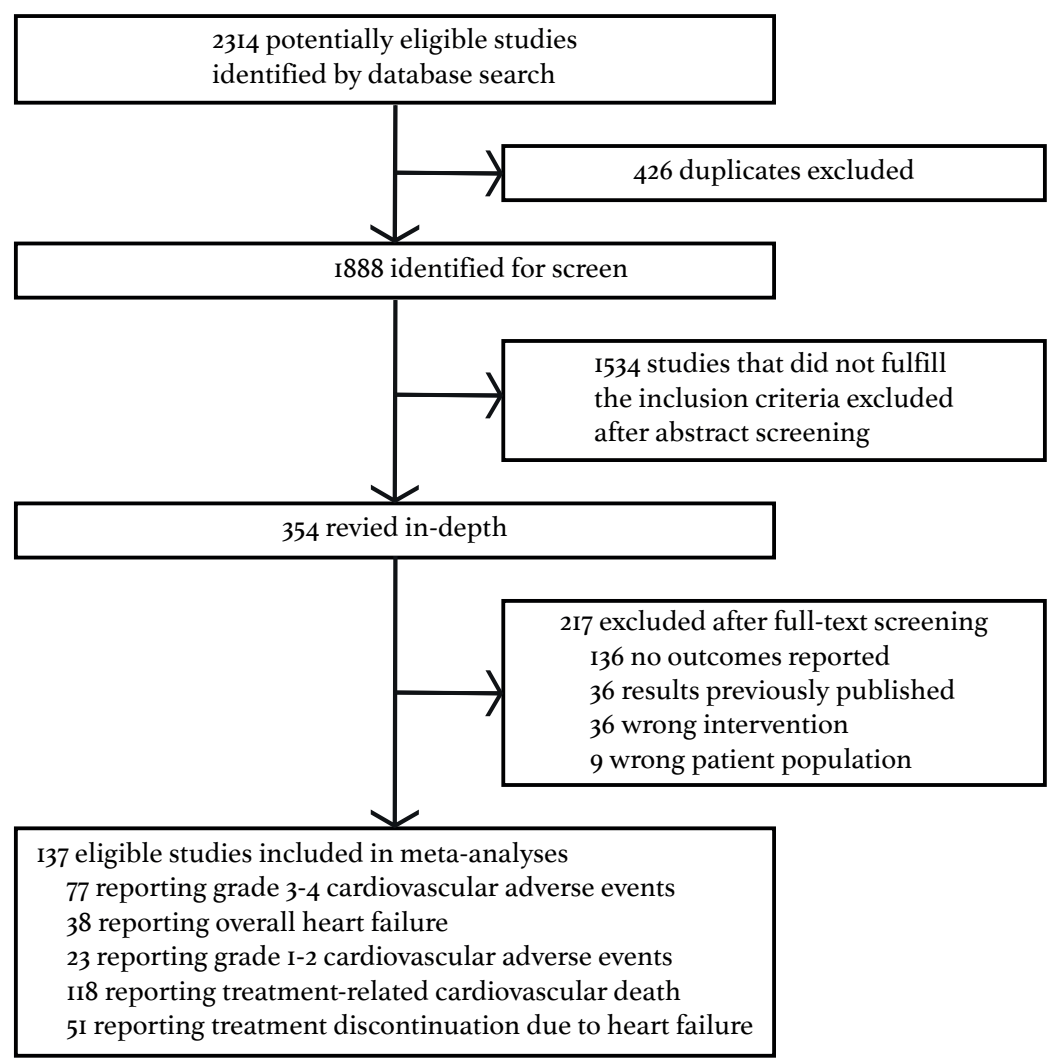

Figure I Study selection 
Standard doses of doxorubicin $\left(50 \mathrm{mg} / \mathrm{m}^{2}\right.$ per cycle), cyclophosphamide $\left(750 \mathrm{mg} / \mathrm{m}^{2}\right.$ per cycle), and vincristine ( $\mathrm{I} \cdot 4 \mathrm{mg} / \mathrm{m}^{2}$ [maximum $2 \mathrm{mg}$ ]) were given in $\mathrm{I} 36(82 \%)$ of 165 subgroups of treatment. 85 subgroups (954I patients) were treated with CHOP, 76 with R-CHOP (II 293 patients), and in four studies both $\mathrm{CHOP}$ and R-CHOP were used without a subdivision in separate groups (377 patients). Most studies investigated the efficacy of CHOP or R-CHOP for the treatment of high-grade non-Hodgkin lymphomas ( 136 subgroups of treatment and I8 535 patients in total). The mean interval between cycles was 19.9 days (SD 3.4) and the mean number of cycles was $6 \cdot 3(\mathrm{I} \cdot 3)$. Overall, the median follow-up of the included studies

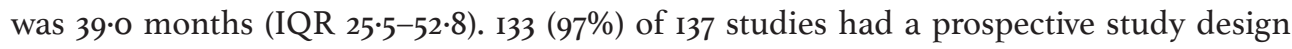
and four (3\%) studies were retrospective. I03 (75\%) studies reported adverse events using a well-established toxicity grading system and $29(21 \%)$ reported adverse events in grades but didn't specify which grading system was used. $85(62 \%)$ studies reported on the occurrence of cardiovascular adverse events. Only 6 (4\%) studies specified the incidence of heart failure or left ventricular dysfunction as a primary or secondary outcome. Cardiac dysfunction was specified as an exclusion criterion for CHOP or R-CHOP therapy in 58 (42\%) of I37 studies.

96 subgroups of treatment were derived from 77 (56\%) of 137 studies that reported on the proportion of patients with high-grade (grade 3-4) cardiovascular adverse events. Of the 60 excluded studies, three studies did not report adverse events, 30 did not report all adverse events, and for 27 studies completeness of reporting could not be confirmed. I4 35I patients were included in the meta- analysis, for which a random-effects model was used (heterogeneity test $\mathrm{Q}=326 \cdot 2 \mathrm{I} ; \tau^{2}=0 \cdot 0042 ; \mathrm{I}^{2}=7 \mathrm{I} \cdot 40 \%$; $\mathrm{p}<0 \cdot 000 \mathrm{I}$ ). The proportion of grade $3-4$ cardiovascular adverse events ranged from $0 \cdot 0 \%$ to $15 \cdot 1 \%$, with a pooled proportion of $2 \cdot 35 \%$ (95\% CI I.8I-2·93; Figure 2).

38 studies, covering 47 subgroups of treatment and 5936 patients, reported on the occurrence of overall heart failure. According to the random-effects model (heterogeneity test: $\mathrm{Q}=527 \cdot 33, \tau^{2}=0 \cdot 0384 ; \mathrm{I}^{2}=95 \cdot 05 \%$; $\left.<<0 \cdot 000 \mathrm{I}\right)$ the pooled proportion of overall heart

\begin{tabular}{|c|c|c|c|c|}
\hline \multicolumn{3}{|c|}{ Events/Total } & \multirow{2}{*}{$\begin{array}{c}\text { Proportion }(95 \% \mathrm{CI}) \\
1.31(0.16-4 \cdot 64)\end{array}$} & \multirow{2}{*}{$\begin{array}{c}\text { Weight } \\
1 \cdot 2 \%\end{array}$} \\
\hline Gams et al (1985) 31 & $2 / 153$ & +1: & & \\
\hline Gordon et al (1992) 32 & $4 / 174$ & & $2.30(0.63-5.78)$ & $1.3 \%$ \\
\hline Pavlovsky et al (1992)33 & $0 / 44$ & 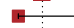 & $0.00(0.00-8.04)$ & $0.7 \%$ \\
\hline Smalley et al $(1992)^{34}$ & $7 / 249$ & & $2 \cdot 81(1 \cdot 14-5 \cdot 71)$ & $1.4 \%$ \\
\hline Yahalom et al (1993) 35 & $0 / 18$ & 1 & $0.00(0.00-18.53)$ & $0.4 \%$ \\
\hline Cooper et al (1994) 36 & $2 / 111$ & + & $1.80(0.22-6 \cdot 36)$ & $1 \cdot 1 \%$ \\
\hline Zinzani et al (1995) ${ }^{37}$ & $1 / 31$ & & $3.23(0.08-16.70)$ & $0.6 \%$ \\
\hline Smith et al $(1996)^{38}$ & $0 / 28$ & 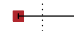 & $0.00(0.00-12.34)$ & $0.5 \%$ \\
\hline Tirelli et al (1998) & $3 / 60$ & & $5.00(1.04-13.92)$ & $0.8 \%$ \\
\hline Ezzat et al $(2000)^{40}$ & $0 / 51$ & 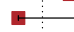 & $0.00(0.00-6.98)$ & $0.8 \%$ \\
\hline Giles et al (2000) ${ }^{41}$ & $0 / 214$ & 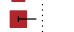 & $0.00(0.00-1.71)$ & $1.3 \%$ \\
\hline Giles et al $(2000)^{41}$ & $7 / 221$ & & $3 \cdot 17(1 \cdot 28-6 \cdot 42)$ & $1 \cdot 3 \%$ \\
\hline Jacobson et al $(2000)^{42}$ & $0 / 20$ & 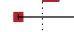 & $0.00(0.00-16.84)$ & $0.4 \%$ \\
\hline Mead et al $(2000)^{43}$ & $1 / 32$ & $\longrightarrow$ & $3.12(0.08-16.22)$ & $0.6 \%$ \\
\hline Niitsu et al (2001) 44 & $1 / 40$ & 案 & $2.50(0.06-13.16)$ & $0.7 \%$ \\
\hline Vose et al $(2001)^{45}$ & $1 / 33$ & & $3.03(0.08-15.76)$ & $0.6 \%$ \\
\hline Coiffier et al (2002) ${ }^{9}$ & $16 / 202$ & & $7.92(4.60-12 \cdot 54)$ & $1.3 \%$ \\
\hline Coiffier et al $(2002)^{9}$ & $16 / 197$ & & $8 \cdot 12(4 \cdot 71-12 \cdot 85)$ & $1.3 \%$ \\
\hline Yamazaki et al (2002) ${ }^{46}$ & $0 / 25$ & 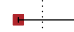 & $0.00(0.00-13.72)$ & $0.5 \%$ \\
\hline Yamazaki et al (2002) 46 & $0 / 14$ & \pm & $0.00(0.00-23.16)$ & $0.3 \%$ \\
\hline Blayney et al (2003) 47 & $5 / 103$ & & $4.85(1.59-10.97)$ & $1 \cdot 1 \%$ \\
\hline Doorduijn et al (2003) 48 & $15 / 389$ & & $3.86(2 \cdot 17-6 \cdot 28)$ & $1.5 \%$ \\
\hline Ösby et al (2003) 49 & $8 / 205$ & + & $3.90(1.70-7.54)$ & $1.3 \%$ \\
\hline Tilly et al $(2003)^{50}$ & $11 / 312$ & & $3.53(1.77-6.22)$ & $1.4 \%$ \\
\hline
\end{tabular}




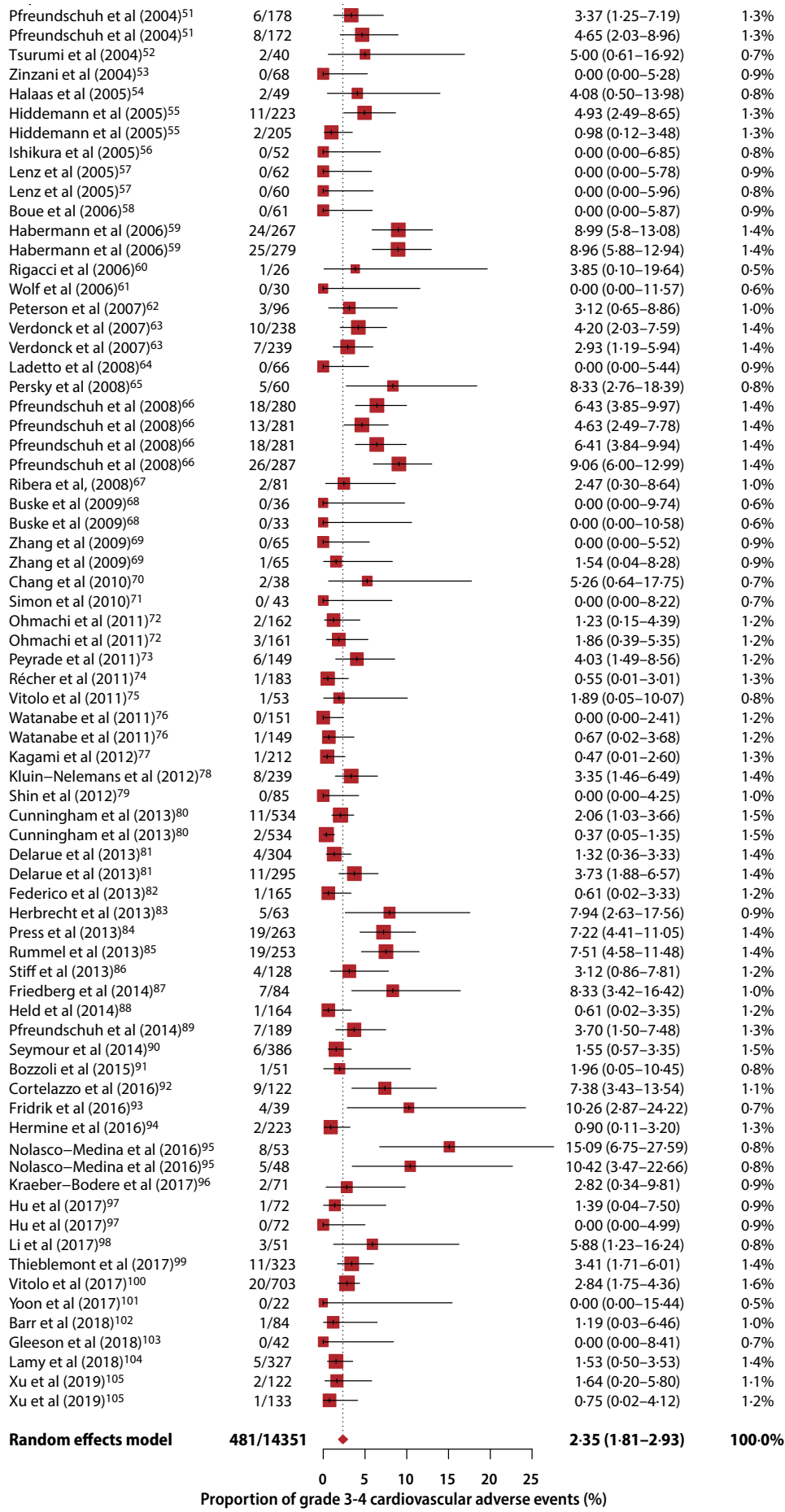

Figure 2 Pooled proportion of patients with grade 3-4 cardiovascular adverse events 
failure was $4 \cdot 62 \%(95 \%$ CI $2 \cdot 25-7 \cdot 65)$.

From a meta-analysis of 30 subgroups of treatment derived from 23 studies that reported the proportion of grade I-2 cardiovascular adverse events (4688 patients), we found the pooled proportion of grade I-2 cardiovascular adverse events to be $8.52 \%$ (95\% CI 5.58-II.96). Cause of death was specified in I34 subgroups of treatment (I5 055 patients), in which the proportion of (possible) treatment-related cardiac death ranged from $0 \cdot 0 \%$ to $5 \cdot 6 \%$; based on a fixed-effect model, the pooled proportion was $0 \cdot 03 \%$ (0-0.IO). No relation between the proportion of patients who died of treatment-related cardiac death and time of follow-up was detected $(\mathrm{r}=0 \cdot 104 ; \mathrm{p}=0 \cdot 50)$. Treatment discontinuation due to heart failure showed similar results, with a proportion of less than $0 \cdot 0001 \%(0-0 \cdot 05)$, on the basis of a meta-analysis of 56 subgroups of treatment ( 3802 patients), done with a fixed-effects model. The outcome of all meta-analyses using both the fixed-effect and random-effects models are presented in Supplemental File 2.

Subgroup analysis of grade 3-4 cardiovascular adverse events showed a significant influence of age, with an increased risk for studies including patients with a median age of 65 years or older compared with studies including patients with a median age of less than 65 years (Table I). Also the variable sex showed a significant effect, with a higher proportion of patients with high-grade cardiovascular adverse events in studies with a larger proportion of females (Table I). Other subgroup analyses (ie, whether or not cardiac screening was performed after the completion of chemotherapy, CHOP vs R-CHOP, number of cycles, and interval between cycles) did not generate any significant results on the percentage of patients with grade $3-4$ cardiovascular adverse events (Table I).

Subgroup analysis of studies that reported on the occurrence of heart failure (44 subgroups of treatment, 5643 patients) showed a significant difference in the reported proportion of patients with overall heart failure when active screening of cardiac function was done after CHOP or R-CHOP treatment was finished (Table I; Figure 3). Furthermore, we found an increased proportion of patients with overall heart failure when more CHOP or R-CHOP cycles were given (ie, increased cumulative doxorubicin and cyclophosphamide dose). There was no effect of R-CHOP vs CHOP, interval between cycles, age, and sex (Table $\mathbf{I}$ ). We also found no relation between the reported proportion of patients with overall heart failure and time of follow-up.

Risk of bias and quality of reporting of harms was individually assessed for all studies. Conservatively, we judged all studies for which we could not extract complete information on all adverse events to be at risk of selective reporting and incomplete outcome data. $53(39 \%)$ of 137 studies were rated as high risk of bias for incomplete outcome data and $54(39 \%)$ for selective reporting. The quality assessment revealed that the reporting of harms was of good quality in $28(20 \%)$ studies, moderate in $98(72 \%)$, and poor in II $(8 \%)$.

The $\mathrm{Q}$ test for heterogeneity was significant in three meta-analyses, supporting the use of a random-effects model. As a sensitivity analysis, the results of a fixed-effects model are provided in Supplemental File 2. 
Table I Subgroup analysis of grade 3-4 cardiovascular events and overall heart failure

\begin{tabular}{|c|c|c|c|c|c|}
\hline & $\begin{array}{l}\text { Subgroups of } \\
\text { treatment (n) }\end{array}$ & $\begin{array}{c}\text { Regression } \\
\text { coefficient } \\
(95 \% \mathrm{CI})\end{array}$ & $\begin{array}{c}\text { Pooled } \\
\text { proportion } \\
(95 \% \mathrm{CI})\end{array}$ & $\begin{array}{l}\text { Relative risk } \\
\quad(95 \% \mathrm{CI})\end{array}$ & p value \\
\hline \multicolumn{6}{|c|}{ Grade 3-4 cardiovascular adverse events } \\
\hline $\begin{array}{l}\text { Treatment } \\
\text { R-CHOP } \\
\text { CHOP }\end{array}$ & $\begin{array}{l}49 \\
45\end{array}$ & & $\begin{array}{l}2.67 \%(\mathrm{I} .84 \text { to } 3.62) \\
2.105(\mathrm{I} .44 \text { to } 2.87)\end{array}$ & $\begin{array}{l}\text { I.27 (I.02 to } \\
\text { I. } 58)\end{array}$ & $0.33^{*}$ \\
\hline $\begin{array}{l}\text { Screening } \\
\text { No } \\
\text { Yes }\end{array}$ & $\begin{array}{c}85 \\
8\end{array}$ & & $\begin{array}{l}2.35 \%(\mathrm{I} .79 \text { to } 2.98) \\
2.38 \%(0.76 \text { to } 4.67)\end{array}$ & & $0.98^{*}$ \\
\hline $\begin{array}{l}\text { Number of cycles } \\
\quad>6 \text { (high) } \\
\leq 6 \text { (low) }\end{array}$ & $\begin{array}{l}94 \\
53 \\
4 \mathrm{I}\end{array}$ & $\begin{array}{c}0.7 \mathrm{I} \\
(-0.65 \text { to } 2.07)\end{array}$ & $\begin{array}{l}2.06 \%(\mathrm{I} .4 \mathrm{O} \text { to } 2.82) \\
2.45 \%((\mathrm{I} .69 \text { to } 3.33)\end{array}$ & $\begin{array}{l}\text { I.I9 (0.95 to } \\
\text { I. } 49)\end{array}$ & $\begin{array}{l}0.30 \dagger \\
0.48^{*}\end{array}$ \\
\hline $\begin{array}{l}\text { Interval between } \\
\text { cycles (days) } \\
\geq 2 \text { I (long) } \\
<2 \text { I (short) }\end{array}$ & $\begin{array}{l}25 \\
68\end{array}$ & $\begin{array}{c}0.24 \\
(-0.70 \text { to } 0.20)\end{array}$ & $\begin{array}{l}2.64 \% \text { (I.69 to } 3.76) \\
2.34 \%(\mathrm{I} .69 \text { to } 3.07)\end{array}$ & $\begin{array}{l}\text { I.I3 (0.9I to } \\
\text { I.40) }\end{array}$ & $\begin{array}{l}0.29 \dagger \\
0.48^{*}\end{array}$ \\
\hline $\begin{array}{l}\text { Age (years) } \\
\begin{array}{l}\geq 65 \text { (old) } \\
<65 \text { (young) }\end{array}\end{array}$ & $\begin{array}{l}91 \\
58 \\
33\end{array}$ & $\begin{array}{c}0.39 \\
\text { (o.2I to } 0.57)\end{array}$ & $\begin{array}{l}\text { I. } 38 \%(0.89 \text { to } 1.96) \\
4.23 \%(3.28 \text { to } 5.50)\end{array}$ & $\begin{array}{c}3.18(2.54 \text { to } \\
3.98)\end{array}$ & $\begin{array}{l}<0.0001 \dagger \\
<0.000 I^{*}\end{array}$ \\
\hline $\begin{array}{l}\text { Sex (\% of female } \\
\text { participants })^{t}\end{array}$ & 92 & $\begin{array}{c}0.24 \\
(0.09 \text { to } 0.40)\end{array}$ & & & $0.0023 \dagger$ \\
\hline & & Overall hes & ailure & & \\
\hline $\begin{array}{l}\text { Treatment } \\
\text { R-CHOP } \\
\text { CHOP }\end{array}$ & $\begin{array}{l}22 \\
24\end{array}$ & & $\begin{array}{l}5.57 \%(\text { I. } 59 \text { to II. } 36) \\
3.24 \%(\text { I.09 to } 6.22)\end{array}$ & $\begin{array}{c}\mathrm{I} .72 \\
(\mathrm{I} .33 \text { to } 2.2 \mathrm{I})\end{array}$ & $0.39^{*}$ \\
\hline $\begin{array}{l}\text { Screening } \\
\text { No } \\
\text { Yes }\end{array}$ & $\begin{array}{l}30 \\
\mathrm{I} 4\end{array}$ & & $\begin{array}{l}\text { I. } 64 \%(0.82 \text { to } 2.65) \\
\text { II. } 72 \%(3.00 \text { to } 24.53)\end{array}$ & & $0.017^{*}$ \\
\hline $\begin{array}{l}\text { Number of cycles } \\
>6 \text { (high) } \\
\leq 6 \text { (low) }\end{array}$ & $\begin{array}{l}46 \\
\text { I5 } \\
3 \mathrm{I}\end{array}$ & $\begin{array}{c}4.99 \\
\text { (o.66 to 9.32) }\end{array}$ & $\begin{array}{c}6.75 \%(2.66 \text { to } 12.29) \\
3.8 \mathrm{I} \%(\text { I.I6 to } 7.58)\end{array}$ & $\begin{array}{c}\text { I.78 } \\
\text { (I.43 to } 2.23)\end{array}$ & $\begin{array}{l}0.024 \dagger \\
0.3 \mathrm{I}^{*}\end{array}$ \\
\hline $\begin{array}{l}\text { Interval between } \\
\text { cycles (days) }\end{array}$ & 47 & $\begin{array}{c}\mathrm{I} . \mathrm{I} 2 \\
(-0.56 \text { to } 2.8 \mathrm{I})\end{array}$ & & & O.I9 † \\
\hline $\begin{array}{l}\geq 2 \text { I (long) } \\
<2 \text { I (short) }\end{array}$ & $\begin{array}{l}35 \\
12\end{array}$ & & $\begin{array}{l}5.68 \%(2.3 \mathrm{I} \text { to IO.I9) } \\
2.1 \mathrm{II} \%(\text { I.OI to } 3.5 \mathrm{I})\end{array}$ & $\begin{array}{c}2.68 \\
\text { (I.98 to 3.63) }\end{array}$ & $0.057^{*}$ \\
\hline $\begin{array}{l}\text { Age (years) } \\
\geq 65 \text { (old) } \\
<65 \text { (young) }\end{array}$ & $\begin{array}{l}45 \\
\text { I8 } \\
27\end{array}$ & $\begin{array}{c}0.3 \mathrm{I} \\
(-0.42 \text { to } \mathrm{I.07})\end{array}$ & $\begin{array}{l}8.62 \%(2.77 \text { to } 16.89) \\
2.63 \%(0.88 \text { to } 5.05)\end{array}$ & $\begin{array}{c}3.27 \\
\text { (2.54 to } 4.20)\end{array}$ & $\begin{array}{l}0.42 \dagger \\
0.068^{*}\end{array}$ \\
\hline $\begin{array}{l}\text { Sex }(\% \text { of female } \\
\text { participants })^{+}\end{array}$ & 42 & $\begin{array}{c}0.26 \\
(-0.48 \text { to } 0.99)\end{array}$ & & & $0.50 \dagger$ \\
\hline
\end{tabular}


In post-hoc analyses, we found no correlation between median follow-up times and the reported proportion of patients with overall heart failure and treatment-related cardiac death $(r=-0 \cdot 08 I ; p=0 \cdot 37)$. For studies that reported on heart failure, we found no differences in study characteristics and patient demographics between studies that did and did not assess cardiac function after chemotherapy (Supplemental File 3a). The reported proportion of patients with overall heart failure was not significantly different between studies that did and did not handle cardiac dysfunction as an exclusion criterion for CHOP or R-CHOP treatment (Supplemental File 3b). We found no association between the number of high-grade cardiovascular adverse events and overall mortality.

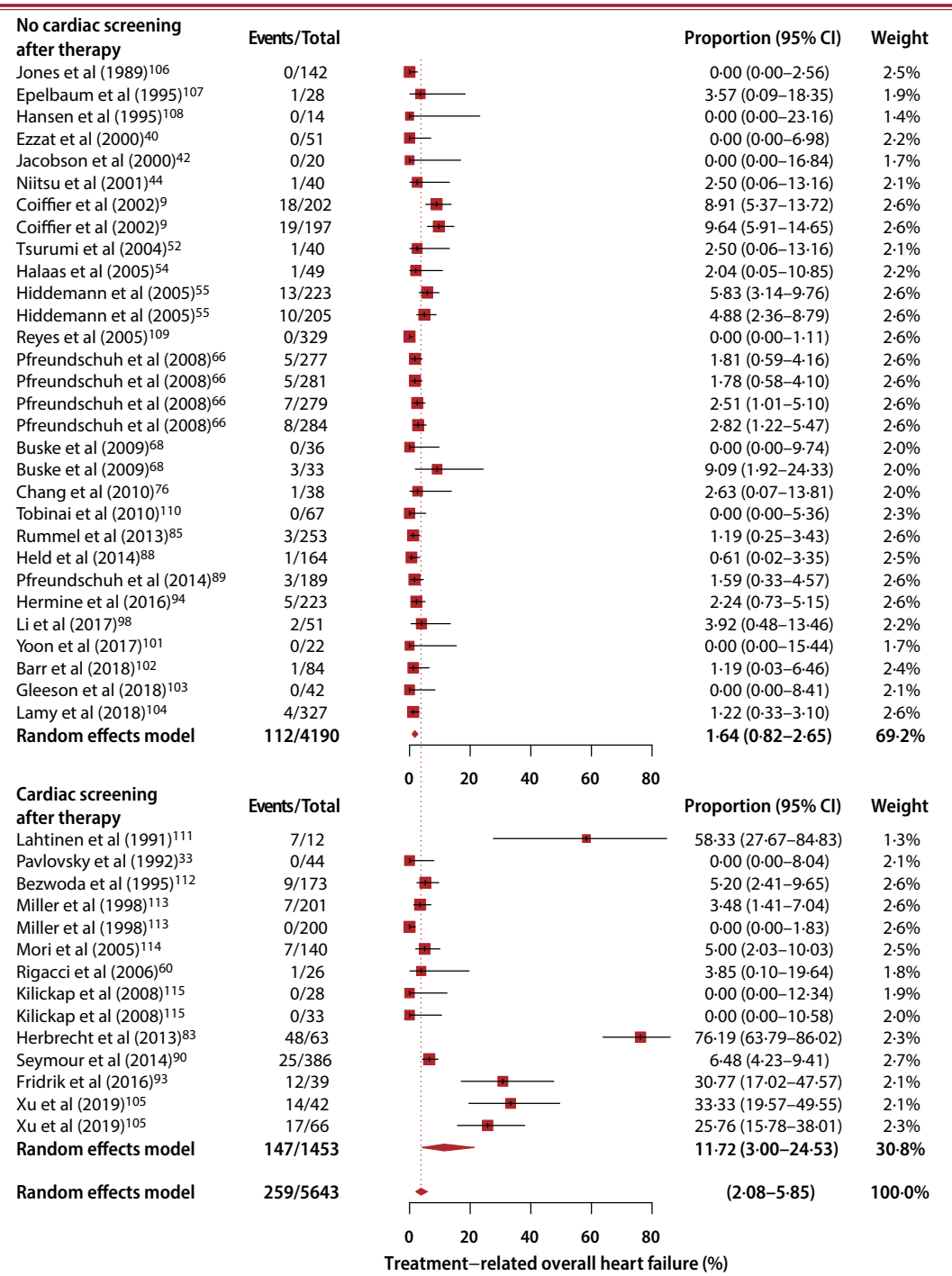

Figure 3 Pooled proportion of patients with overall heart failure, by studies with and without cardiac screening after therapy 


\section{Discussion}

To our knowledge, this is the first study to comprehensively evaluate the proportion of patients that develop cardiovascular adverse events in the context of the CHOP and R-CHOP regimen. Previous work has mainly focused on unravelling the cardiovascular toxicity of individual drugs, but we believe that assessing the toxicity of complete treatment regimens is necessary, because of their increasing complexity and possible toxic synergistic interactions between compounds. The most important findings of this work are: the reported proportion of patients that develop severe cardiovascular adverse events is quite low (pooled proportion of $2 \cdot 35 \%$ ); female sex and older age ( $\geq 65$ years) are independently associated with an increased risk of severe cardiovascular adverse events; the reported proportion of patients with overall heart failure increased significantly from I $64 \%$ to II $\cdot 72 \%$ when cardiac function was screened actively at the end of treatment; and discontinuation of CHOP or $\mathrm{R}-\mathrm{CHOP}$ due to treatment-related heart failure is rare.

In previous studies among paediatric cancer survivors, female sex has repeatedly been associated with an increased risk of anthracycline-related left ventricular dysfunction [II6]. In adults, this relationship has been inconclusive, probably because most studies of cardiotoxicity have been done in female patients with breast cancer. Moreover, we confirmed that older individuals have an increased risk of developing high-grade cardiovascular adverse events. This elevated risk might be provoked by a high prevalence of cardiovascular risk factors in this patient population, as shown in a large series of patients with lymphoma aged 65 years or older, among whom $73 \%$ had hypertension, $54 \%$ hyperlipidaemia, and $32 \%$ diabetes [23]. The patients that developed severe cardiovascular adverse events in our meta-analysis might have had pre-existing or ongoing cardiac disease, which could have increased their susceptibility to these unintended effects in the context of a second hit-like phenomenon [13,I4,II7].

Considering the increasing prevalence of non-Hodgkin lymphoma with age, drugs that are less cardiotoxic could be considered. The antineoplastic potency of anthracycline drugs has been assumed to be proportional to the acute haematological toxicity and by extension to toxic effects on other organs, including the heart [II8,II9]. From this, conversion factors for the cardiotoxicity of different anthracyclines have been derived. The validity of this assumption was investigated in a large cohort of childhood cancer survivors, which estimated the conversion factor of epirubicin to doxorubicin at $0 \cdot 8$, indicating that this compound is associated with reduced cardiotoxicity [II9]. However, the first trial [I20] comparing a regimen composed of cyclophosphamide, vincristine, prednisone, and epirubicin $\left(70 \mathrm{mg} / \mathrm{m}^{2}\right)$ with or without rituximab ([R]-CEOP70) versus standard CHOP or $\mathrm{R}-\mathrm{CHOP}$ in 348 adults with diffuse large B-cell lymphoma or follicular lymphoma did not report a difference in the proportion of patients with a decline by to percentage points or more in left-ventricular ejection fraction (LVEF), although the number of patients with raised troponin levels was significantly lower in the (R-)CEOP70 group. The oncological efficacy of these regimens was comparable. Additionally, in a multicentre, phase 3, randomised, controlled trial [105] done in China in 2019, 3 years after remission $13 \%$ of patients treated with R-CEOP7O developed a LVEF decline of more than Io percentage points 
compared with $29 \%$ of patients treated with R-CHOP50. Additional studies with longer follow-up are necessary to assess the effect of substituting doxorubicin with epirubicin on the incidence of hard clinical endpoints, including symptomatic heart failure.

Besides epirubicin, pixantrone (an analogue of mitoxantrone) could also be less cardiotoxic than doxorubicin. This drug was conditionally approved by the European Medicine Agency in 2012, and is currently indicated as monotherapy for the treatment of adult patients with multiply relapsed or refractory aggressive B-cell non-Hodgkin lymphoma. Thus far, only one trial [83] has investigated this drug as first-line therapy, comparing $\mathrm{R}-\mathrm{CHOP}$ with a modified $\mathrm{R}-\mathrm{CHOP}$ regimen in which doxorubicin was replaced by pixantrone (R-CPOP) in I4O patients with diffuse large B-cell lymphoma. The proportion of patients who had complete or unconfirmed complete response to treatment was lower in the R-CPOP group (75\%) than in the R-CHOP group (84\%), but similar progression-free survival and event-free survival were reported. The R-CPOP regimen was substantially less cardiotoxic, with $2 \%$ of patients in this group developing an LVEF decline of 20 percentage points or more versus $\mathrm{I} 7 \%$ in the R-CHOP group, and no patients in the R-CPOP group developing symptomatic heart failure versus $6 \%$ in the $\mathrm{R}-\mathrm{CHOP}$ group. The authors suggested that pixantrone might be an alternative in patients at high risk of cardiotoxicity, with this possibility currently being investigated in a phase 2 trial in older patients with diffuse large B-cell lymphoma and decreased cardiac function (EudraCT number 20I4-005069$60)$.

The pooled proportion of patients developing heart failure $(4 \cdot 62 \%)$ in our meta-analysis is in line with previous studies reporting on the development of symptomatic heart failure, with a 3-5\% incidence at a cumulative doxorubicin dose of $400 \mathrm{mg} / \mathrm{m}^{2}$ [I2,I2I]. Many patients in these studies had also received concomitant treatment with cyclophosphamide, complicating the interpretation of the association of this individual agent with left ventricular dysfunction. However, a large cohort study [24] in patients with lymphoma comparing the 5-year cumulative risk of symptomatic heart failure between patients treated with ( $\mathrm{n}=1994)$ or without $(\mathrm{n}=446)$ anthracyclines, found that the risk was only $0 \cdot 8 \%$ in the patients not exposed to anthracyclines versus $4 \cdot 5-7 \cdot 9 \%$ in the anthracycline group. Patients treated without anthracyclines had received similar cyclophosphamide doses as incorporated in CHOP and R-CHOP, indicating that it does not contribute considerably to the cardiovascular toxicity of $\mathrm{CHOP}$ and R-CHOP at these low doses. From the small number of studies that have described an association between cyclophosphamide and left ventricular dysfunction, doses greater than $\mathrm{I} \cdot 5 \mathrm{~g} / \mathrm{m}^{2}$ per day seem to be associated with a high risk of heart failure [I6].

Cardiac dysfunction was a common exclusion criterion for CHOP and R-CHOP, applied in $58(42 \%)$ of 137 studies. However, strikingly, only 38 (28\%) studies reported on the incidence of heart failure and an even smaller number performed active cardiac screening before and after chemotherapy to determine whether left ventricular dysfunction had been induced. The significant increase in the reported proportion of patients with cardiac dysfunction from $\mathrm{I} \cdot 64 \%$ to $\mathrm{I} \cdot 72 \%$ when patients received cardiac follow-up post chemothe- 
rapy is suggestive of a widespread underdiagnosis of this complication. An important contributing factor could be the common practice of solely reporting the incidence of high-grade adverse events in phase 3 clinical trials, that are collected during a limited period of time [I22]. In the current CTCAE (version 5.0), only patients with heart failure symptoms at rest or patients with a symptomatic drop in the LVEF are counted as severe. These criteria are problematic, as heart failure symptoms can be easily misattributed to non- cardiac adverse events in patients with cancer [123] and heart failure symptoms are known to correlate poorly with LVEF. A considerable decline in cardiac contractility can be evoked by cardiotoxic chemotherapeutics without the immediate onset of symptomatic heart failure. Most patients develop this decline within the first year of receiving anthracycline-containing therapy [I24]. Systolic dysfunction can thereafter progress silently for years by the activation of various compensatory mechanisms, including peripheral vasoconstriction and activation of the renin-angiotensin aldosterone system [I25].

Indeed, we found that onset of symptomatic heart failure during $\mathrm{CHOP}$ or $\mathrm{R}-\mathrm{CHOP}$ treatment leading to treatment discontinuation is very rare. In addition, the reported proportion of patients with left ventricular dysfunction was limited to I.64\% in studies that did not evaluate cardiac function after chemotherapy. Clinical heart failure is mainly a concern in the long-term, as indicated by other studies in lymphoma survivors, with an increasing cumulative risk from $0 \cdot 6-4 \cdot 0 \%$ at I year to $5 \cdot 2-9 \cdot 4 \%$ at 8 years after treatment termination [24]. Patients that have developed clinical heart failure because of anthracyclines generally have a poor prognosis with a cardiovascular mortality of $9 \%$ at 5 years and $24 \%$ at Io years [I26]. An early discovery of a decline in cardiac function in the presymptomatic phase and immediate initiation of conventional heart failure therapy gives the greatest chance of complete functional recovery [I27]. Treatment involves management according to standard heart failure guidelines with the administration of angiotensin-converting enzyme (ACE) inhibitors and beta blockers, both targeting the renin-angiotensin aldosterone system to counteract adverse cardiac remodeling [I28].

This study has several limitations. First, we did not have access to individual patient data (IPD), which has multiple benefits over pooling aggregate data [I29]. In the absence of IPD, it was not possible to do competing risk analyses or measure the effect of known cardiovascular risk factors, including smoking, diabetes, hypertension, and chest radiation on cardiovascular adverse event incidence. Although we observed that female sex and older age were associated with an increased risk of severe cardiovascular events in subgroup analyses, some caution is required when interpreting these results, which are based on summary estimates. However, significant findings detected with aggregate data are replicated in $95 \%$ of IPD meta-analyses [I30].

Second, despite using well defined inclusion criteria, some level of heterogeneity was expected because of differences in study designs and populations. Based on the Q test, significant heterogeneity was present in three meta-analyses (Supplemental File 2). As this test can be too sensitive when used to analyse results from a large number of studies, the random-effects model was selected, on the basis of visual evaluation of the heterogeneity in the forest plot, in combination with the $\tau^{2}$ and $\mathrm{I}^{2}$ statistics. 
Third, because of the risk of bias induced by selective reporting of adverse events, studies could have been excluded from the meta-analyses regardless of whether cardiovascular adverse events occurred. Of 60 studies that were not included in the meta-analysis of grade 3-4 cardiovascular adverse events, three studies did not report adverse events, 30 did not report on all adverse events, and for 27 studies completeness of reporting could not be confirmed.

Fourth, the outcome of this meta-analysis is likely to be influenced by the inconsistency of toxicity grading systems of cardiovascular adverse events. For example, with the CTCAE, a (large) asymptomatic decline in cardiac function can be reported as grade $0-3$ depending on the entry chosen by the researcher [123]. Furthermore, cardiotoxicity is used as an umbrella term for all manifestations of cardiovascular toxicity by some researchers, whereas others use this term only for chemotherapy-induced left ventricular dysfunction. Because of poor cardiovascular adverse event specification (appendix p I4), a small number of studies could be included in the meta-analysis of overall heart failure and a separate analysis for subclinical versus clinical heart failure or other cardiovascular adverse events were not possible. The discrepancy between the proportion of patients with high-grade cardiovascular adverse events and the proportion with overall heart failure could be partly explained by cases of subclinical heart failure. We hypothesised that studies that did cardiac screening before and after chemotherapy might have included patients at an increased risk of heart failure. However, on the basis of the summary estimates, we did not find any differences between the studies that could explain the large difference in the proportion of patients reported with left ventricular dysfunction between studies that did and did not perform cardiac screening (appendix p 26). There were also no differences in the proportion of patients with cardiovascular adverse events in the studies that handled cardiac dysfunction as an exclusion criterion and studies that did not (appendix p 26). Moreover, we could not establish a relationship between overall survival and the proportion of patients having cardiovascular adverse events, presumably because of the short period that clinical trials record adverse events and the varying prognosis of different non-Hodgkin lymphoma subtypes. Finally, we did not contact authors of the original studies to assess the accuracy and veracity of our extracted data.

We believe that the findings of this study are important in raising the awareness of cardiovascular adverse events in patients with non-Hodgkin lymphoma treated with CHOP or R-CHOP. Our results can presumably also be generalised to non-Hodgkin lymphoma patients receiving a regimen of dose-adjusted etoposide, prednisone, vincristine, cyclophosphamide, and doxorubicin with or without rituximab (DA-EPOCH[-R]), since the regimen contains comparable doses of doxorubicin. To reduce the burden of cardiovascular diseases in patients with cancer and survivors, close multidisciplinary collaborations between (haemato-)oncologists and cardiologists are currently coordinated by the implementation of cardio- oncology clinics worldwide [13I,I32]. These clinics will provide more insight into the burden of cardiovascular adverse events in a real-world setting and aid in the implementation of targeted strategies for their primary and secondary prevention. The optimal strategy might vary according to cancer type, as the prognosis of the malignancy 
is often the dominant factor. Serial echocardiographic assessment is currently the cornerstone in the detection of early changes in LVEF because of its availability and versatility [133]. In patients exposed to anthracyclines, the duration of cardiac monitoring should be at least I year, as almost all patients that will develop left ventricular dysfunction display an important decline in cardiac function within the first year [124]. In addition to standard LVEF measurements, deformation imaging by means of global longitudinal strain has shown promising results to detect early changes in myocardial contractility in this setting [134]. Other screening methods include biomarkers, of which the most promising is troponin with a negative predictive value of $93 \%$ [135]. For primary prevention, ACE inhibitors and beta blockers have been studied in patients with breast cancer in a small number of trials with small sample size [136,137]. Moreover, the bisdioxopiperazine drug dexrazoxane warrants further investigation. A Cochrane meta-analysis [138] pooling data from eight trials showed a significant and clinically relevant benefit in favour of dexrazoxane for the prevention of clinical heart failure and overall heart failure.

Prospective registries are necessary for the identification of risk factors predisposing patients to cardiovascular adverse events, optimisation of cardiologic screening strategies, and measurement of the progression rate from asymptomatic to clinical heart failure. For future clinical trials within the field of oncology, the type of cardiovascular adverse events should be specified in more detail, since their therapeutic management, reversibility, and prognosis varies. The CONSORT extension for harms can aid researchers herein [28]. Furthermore, we believe that revision of the CTCAE is of paramount importance to assure consistent cardiovascular adverse event grading. Preferably, these revisions should also reflect the duration, reversibility, and multiplicity of adverse events [122,139].

Our systematic review and meta-analysis shows that the proportion of patients who develop severe cardiovascular adverse events after first-line CHOP and R-CHOP treatment is small during the adverse event registration period. Our subgroup analyses suggest that women and older patients have a particularly high risk of severe cardiovascular adverse events, and cardiac screening in these patients is warranted. Despite the well-established association of doxorubicin with left ventricular dysfunction, cardiac function is seldom monitored. However, if assessed post-chemotherapy, cardiac dysfunction is reported in more than $10 \%$ of patients with non-Hodgkin lymphoma suggesting this is a common adverse event that often remains undetected. 


\section{References}

I Ferlay J, Colombet M, Soerjomataram I, et al. Estimating the global cancer incidence and mortality in 20I8: GLOBOCAN sources and methods. Int J Cancer 2019; I44: I94I-53.

2 National Cancer Institute. SEER cancer statistics review, 1975-2015. https://seer.cancer.gov/csr/19752015/ (accessed Aug I2, 2019).

3 Moser EC, Noordijk EM, van Leeuwen FE, et al. Long-term risk of cardiovascular disease after treatment for aggressive non-Hodgkin lymphoma. Blood 2006; I07: 2912-I9.

4 Strongman H, Gadd S, Matthews A, et al. Medium and long-term risks of specific cardiovascular diseases in survivors of 20 adult cancers: a population-based cohort study using multiple linked UK electronic health records databases. Lancet 20I9; 394: IO4I-54.

5 Koene RJ, Prizment AE, Blaes A, Konety SH. Shared risk factors in cardiovascular disease and cancer. Circulation 2016; I33: IIO4-I4.

6 Ewer MS, Ewer SM. Cardiotoxicity of anticancer treatments. Nat Rev Cardiol 20I5; I2: 547-58.

7 McKelvey EM, Gottlieb JA, Wilson HE, et al. Hydroxyldaunomycin (adriamycin) combination chemotherapy in malignant lymphoma. Cancer 1976; 38: I484-93.

8 Fisher RI, Gaynor ER, Dahlberg S, et al. Comparison of a standard regimen (CHOP) with three intensive chemotherapy regimens for advanced non-Hodgkin's lymphoma. N Engl J Med 1993; 328: IOO2-06.

9 Coiffier B, Lepage E, Briere J, et al. CHOP chemotherapy plus rituximab compared with CHOP alone in elderly patients with diffuse large-B-cell lymphoma. N Engl J Med 2002; 346: 235-42.

Io Coiffier B, Thieblemont C, Van Den Neste E, et al. Long-term outcome of patients in the LNH-98.5 trial, the first randomized study comparing rituximab-CHOP to standard CHOP chemotherapy in DLBCL patients: a study by the Groupe d'Etudes des Lymphomes de l'Adulte. Blood 20IO; II6: 204045.

II Zamorano JL, Lancellotti P, Rodriguez Muñoz D, et al. 2016 ESC Position Paper on cancer treatments and cardiovascular toxicity developed under the auspices of the ESC Committee for Practice Guidelines: The Task Force for cancer treatments and cardiovascular toxicity of the European Society of Cardiology (ESC). Eur Heart J 2016; 37: 2768-80I.

I2 Von Hoff DD, Layard MW, Basa P, et al. Risk factors for doxorubicin-induced congestive heart failure. Ann Intern Med 1979; 91: 710-I7.

I3 Lotrionte M, Biondi-Zoccai G, Abbate A, et al. Review and meta-analysis of incidence and clinical predictors of anthracycline cardiotoxicity. Am J Cardiol 2013; II2: 1980-84.

I4 Garcia-Pavia P, Kim Y, Restrepo-Cordoba MA, et al. Genetic variants associated with cancer therapyinduced cardiomyopathy. Circulation 2019; I40: 3I-4I.

I5 Braverman AC, Antin JH, Plappert MT, Cook EF, Lee RT. Cyclophosphamide cardiotoxicity in bone marrow transplantation: a prospective evaluation of new dosing regimens. J Clin Oncol 1991; 9: 1215-23.

I6 Goldberg MA, Antin JH, Guinan EC, Rappeport JM. Cyclophosphamide cardiotoxicity: an analysis of dosing as a risk factor. Blood I986; 68: III4-I8.

I7 Gottdiener JS, Appelbaum FR, Ferrans VJ, Deisseroth A, Ziegler J. Cardiotoxicity associated with high-dose cyclophosphamide therapy. Arch Intern Med I98I; I4I: 758-63.

I8 Buja LM, Ferrans VJ, Graw RG Jr. Cardiac pathologic findings in patients treated with bone marrow transplantation. Hum Pathol 1976; 7: 17-45.

I9 Cazin B, Gorin NC, Laporte JP, et al. Cardiac complications after bone marrow transplantation. A report on a series of 63 consecutive transplantations. Cancer 1986; 57: 206I-69.

20 Steinherz LJ, Steinherz PG, Mangiacasale D, et al. Cardiac changes with cyclophosphamide. Med Pediatr Oncol I98I; 9: 4I7-22.

2I Appelbaum F, Strauchen JA, Graw RG Jr, et al. Acute lethal carditis caused by high-dose combination chemotherapy. A unique clinical and pathological entity. Lancet 1976; I: 58-62.

22 Hequet $\mathrm{O}$, Le QH, Moullet I, et al. Subclinical late cardiomyopathy after doxorubicin therapy for lymphoma in adults. J Clin Oncol 2004; 22: 1864-7I.

23 Hershman DL, McBride RB, Eisenberger A, Tsai WY, Grann VR, Jacobson JS. Doxorubicin, cardiac risk factors, and cardiac toxicity in elderly patients with diffuse B-cell non-Hodgkin's lymphoma. J Clin Oncol 2008; 26: 3159-65. 
24 Baech J, Hansen SM, Lund PE, et al. Cumulative anthracycline exposure and risk of cardiotoxicity; a Danish nationwide cohort study of 2440 lymphoma patients treated with or without anthracyclines. Br J Haematol 20I8; I83: 7I7-26.

25 Moher D, Liberati A, Tetzlaff J, Altman DG. Preferred reporting items for systematic reviews and meta-analyses: the PRISMA statement. J Clin Epidemiol 2009; 62: I006-I2.

26 Freeman MF, Tukey JW. Transformations related to the angular and the square root. Ann Math Stat I950; 2I: 607-II.

27 Higgins JPT, Green S. Cochrane handbook for systematic reviews of interventions. Version 5.I.o. London: The Cochrane Collaboration, 20II.

28 Ioannidis JP, Evans SJ, Gøtzsche PC, et al. Better reporting of harms in randomized trials: an extension of the CONSORT statement. Ann Intern Med 2004; I4I: 78I-88.

29 Higgins JP, Thompson SG, Deeks JJ, Altman DG. Measuring inconsistency in meta-analyses. BMJ 2003; 327: 557-60.

30 Viechtbauer W. Conducting meta-analyses in R with the metafor package. J Stat Softw 20I0; $36:$ I-48.

3I Gams RA, Rainey M, Dandy M, Bartolucci AA, Silberman H, Omura G. Phase III study of BCOP v CHOP in unfavorable categories of malignant lymphoma: a Southeastern Cancer Study Group trial. J Clin Oncol 1985; 3: II88-95.

32 Gordon LI, Harrington D, Andersen J, et al. Comparison of a second-generation combination chemotherapeutic regimen (m-BACOD) with a standard regimen (CHOP) for advanced diffuse nonHodgkin's lymphoma. N Engl J Med I992; 327: 1342-49.

33 Pavlovsky S, Santarelli MT, Erazo A, et al. Results of a randomized study of previously-untreated intermediate and high grade lymphoma using CHOP versus CNOP. Ann Oncol 1992; 3: $205-09$.

34 Smalley RV, Andersen JW, Hawkins MJ, et al. Interferon alfa combined with cytotoxic chemotherapy for patients with non- Hodgkin's lymphoma. N Engl J Med I992; 327: I336-4I.

35 Yahalom J, Varsos G, Fuks Z, Myers J, Clarkson BD, Straus DJ. Adjuvant cyclophosphamide, doxorubicin, vincristine, and prednisone chemotherapy after radiation therapy in stage I low-grade and intermediate-grade nonHodgkin lymphoma. Cancer I993; 7I: 2342-50.

36 Cooper IA, Wolf MM, Robertson TI, et al. Randomized comparison of MACOP-B with CHOP in patients with intermediate-grade non-Hodgkin's lymphoma. J Clin Oncol I994; 12: 769-78.

37 Zinzani PL, Martelli M, Storti S, et al. Phase III comparative trial using CHOP vs CIOP in the treatment of advanced intermediate-grade non-Hodgkin's lymphoma. Leuk Lymphoma I995; 19: 329-35.

38 Smith GM, Child JA, Cullen MH, et al. A phase I trial to assess the value of recombinant human granulocyte colony stimulating factor (R-MeTHuG-CSF, filgrastim) in accelerating the dose rate of chemotherapy for intermediate and high-grade non-Hodgkin's lymphoma (NHL). Hematol Oncol I996; I4: I93-20I.

39 Tirelli U, Errante D, Van Glabbeke M, et al. CHOP is the standard regimen in patients $>$ or $=70$ years of age with intermediate-grade and high-grade non-Hodgkin's lymphoma: results of a randomized study of the European Organization for Research and Treatment of Cancer Lymphoma Cooperative Study Group. J Clin Oncol I998; I6: 27-34.

40 Ezzat AA, Ibrahim EM, Stuart RK, et al. Adding high-dose tamoxifen to CHOP does not influence response or survival in aggressive non-Hodgkin's lymphoma: an interim analysis of a randomized phase III trial. Med Oncol 2000; I7: 39-46.

4I Giles FJ, Shan J, Advani SH, et al. A prospective randomized study of Chop versus Chop plus alpha-2B interferon in patients with intermediate and high grade non-Hodgkin's lymphoma: the International Oncology Study Group NHLi Study. Leuk Lymphoma 2000; 40: 95-IO3.

42 Jacobson JO, Grossbard M, Shulman LN, Neuberg D. CHOP chemotherapy with preemptive granulocyte colony-stimulating factor in elderly patients with aggressive non-Hodgkin's lymphoma: a dose-intensity analysis. Clin Lymphoma 2000; I: 2II-I7, discussion 2I8.

43 Mead GM, Bleehen NM, Gregor A, et al. A medical research council randomized trial in patients with primary cerebral non-Hodgkin lymphoma: cerebral radiotherapy with and without cyclophosphamide, doxorubicin, vincristine, and prednisone chemotherapy. Cancer 2000; 89: I35970 . 
44 Niitsu N, Iijima K. Full-dose CHOP chemotherapy combined with granulocyte colony-stimulating factor for aggressive non-Hodgkin's lymphoma in elderly patients: a prospective study. Ann Hematol 2001; 80: 602-06.

45 Vose JM, Link BK, Grossbard ML, et al. Phase II study of rituximab in combination with chop chemotherapy in patients with previously untreated, aggressive non-Hodgkin's lymphoma. J Clin Oncol 200I; 19: 389-97.

46 Yamazaki T, Sawada U, Kura Y, et al. Dose-intensified CHOP (double-CHOP) followed by consolidation with high-dose chemotherapy for high and high-intermediate risk aggressive non-Hodgkin's lymphomas. Leuk Lymphoma 2002; 43: 2117-23.

47 Blayney DW, LeBlanc ML, Grogan T, et al. Dose-intense chemotherapy every 2 weeks with doseintense cyclophosphamide, doxorubicin, vincristine, and prednisone may improve survival in intermediate- and high-grade lymphoma: a phase II study of the Southwest Oncology Group (SWOG 9349). J Clin Oncol 2003; 21: 2466-73.

48 Doorduijn JK, van der Holt B, van Imhoff GW, et al. CHOP compared with CHOP plus granulocyte colony-stimulating factor in elderly patients with aggressive non-Hodgkin's lymphoma. J Clin Oncol 2003; 2I: 304I-50.

49 Ösby E, Hagberg H, Kvaløy S, et al. CHOP is superior to CNOP in elderly patients with aggressive lymphoma while outcome is unaffected by filgrastim treatment: results of a Nordic Lymphoma Group randomized trial. Blood 2003; IOI: 3840-48.

50 Tilly H, Lepage E, Coiffier B, et al. Intensive conventional chemotherapy (ACVBP regimen) compared with standard CHOP for poor-prognosis aggressive non-Hodgkin lymphoma. Blood 2003; IO2: 428489.

5I Pfreundschuh M, Trümper L, Kloess M, et al. Two-weekly or 3-weekly CHOP chemotherapy with or without etoposide for the treatment of elderly patients with aggressive lymphomas: results of the NHL-B2 trial of the DSHNHL. Blood 2004; I04: 634-4I.

52 Tsurumi H, Yamada T, Sawada M, et al. Biweekly CHOP or THP-COP regimens in the treatment of newly diagnosed aggressive non-Hodgkin's lymphoma. A comparison of doxorubicin and pirarubicin: a randomized phase II study. J Cancer Res Clin Oncol 2004; I30: IO7-I3.

53 Zinzani PL, Pulsoni A, Perrotti A, et al. Fludarabine plus mitoxantrone with and without rituximab versus $\mathrm{CHOP}$ with and without rituximab as front-line treatment for patients with follicular lymphoma. J Clin Oncol 2004; 22: 2654-6I

54 Halaas JL, Moskowitz CH, Horwitz S, et al. R-CHOP-I4 in patients with diffuse large B-cell lymphoma: feasibility and preliminary efficacy. Leuk Lymphoma 2005; 46: 54I-47.

55 Hiddemann W, Kneba M, Dreyling M, et al. Frontline therapy with rituximab added to the combination of cyclophosphamide, doxorubicin, vincristine, and prednisone (CHOP) significantly improves the outcome for patients with advanced-stage follicular lymphoma compared with therapy with CHOP alone: results of a prospective randomized study of the German Low-Grade Lymphoma Study Group. Blood 2005; 106: 3725-32.

56 Ishikura S, Tobinai K, Ohtsu A, et al. Japanese multicenter phase II study of CHOP followed by radiotherapy in stage I-II, diffuse large B-cell lymphoma of the stomach. Cancer Sci 2005; 96: 349-52.

57 Lenz G, Dreyling M, Hoster E, et al. Immunochemotherapy with rituximab and cyclophosphamide, doxorubicin, vincristine, and prednisone significantly improves response and time to treatment failure, but not long-term outcome in patients with previously untreated mantle cell lymphoma: results of a prospective randomized trial of the German Low Grade Lymphoma Study Group (GLSG). J Clin Oncol 2005; 23: 1984-92.

58 Boué F, Gabarre J, Gisselbrecht C, et al. Phase II trial of CHOP plus rituximab in patients with HIVassociated non-Hodgkin's lymphoma. J Clin Oncol 2006; 24: 4123-28.

59 Habermann TM, Weller EA, Morrison VA, et al. Rituximab-CHOP versus CHOP alone or with maintenance rituximab in older patients with diffuse large B-cell lymphoma. J Clin Oncol 2006; 24: $3 \mathrm{I} 2 \mathrm{I}-27$. 
6o Rigacci L, Nassi L, Alterini R, et al. Dose-dense CHOP plus rituximab (R-CHOPI4) for the treatment of elderly patients with high-risk diffuse large B cell lymphoma: a pilot study. Acta Haematol 2006; II5: 22-27.

6I Wolf M, Bentley M, Marlton P, et al. Pegfilgrastim to support CHOP-I4 in elderly patients with nonHodgkin's lymphoma. Leuk Lymphoma 2006; 47: 2344-50.

62 Peterson BA, Johnson J, Shipp MA, Barcos M, Gockerman JP, Canellos GP. High dose CHOP: a phase II study of initial treatment in aggressive non-Hodgkin lymphoma. Cancer and Leukemia Group B 935I. Leuk Lymphoma 2007; 48: 870-80.

63 Verdonck LF, Notenboom A, de Jong DD, et al. Intensified I2-week CHOP (I-CHOP) plus G-CSF compared with standard 24-week CHOP (CHOP-2I) for patients with intermediate-risk aggressive non-Hodgkin lymphoma: a phase 3 trial of the Dutch-Belgian Hemato-Oncology Cooperative Group (HOVON). Blood 2007; IO9: 2759-66.

64 Ladetto M, De Marco F, Benedetti F, et al. Prospective, multicenter randomized GITMO/IIL trial comparing intensive (R-HDS) versus conventional (CHOP-R) chemoimmunotherapy in high-risk follicular lymphoma at diagnosis: the superior disease control of R-HDS does not translate into an overall survival advantage. Blood 2008; III: 4004-I3.

65 Persky DO, Unger JM, Spier CM, et al. Phase II study of rituximab plus three cycles of CHOP and involved-field radiotherapy for patients with limited-stage aggressive B-cell lymphoma: Southwest Oncology Group study ooI4. J Clin Oncol 2008; 26: 2258-63.

66 Pfreundschuh M, Schubert J, Ziepert M, et al. Six versus eight cycles of bi-weekly CHOP-I4 with or without rituximab in elderly patients with aggressive $\mathrm{CD} 2 \mathrm{O}+\mathrm{B}$-cell lymphomas: a randomised controlled trial (RICOVER-60). Lancet Oncol 2008; 9: IO5-I6.

67 Ribera JM, Oriol A, Morgades M, et al. Safety and efficacy of cyclophosphamide, adriamycin, vincristine, prednisone and rituximab in patients with human immunodeficiency virus-associated diffuse large B-cell lymphoma: results of a phase II trial. Br J Haematol 2008; I4O: 4II-I9.

68 Buske C, Hoster E, Dreyling M, et al. The addition of rituximab to front-line therapy with CHOP (R-CHOP) results in a higher response rate and longer time to treatment failure in patients with lymphoplasmacytic lymphoma: results of a randomized trial of the German Low-Grade Lymphoma Study Group (GLSG). Leukemia 2009; 23: 153-6I.

69 Zhang Q, Wang J, Yu Z, et al. The effect of biweekly CHOP and standard CHOP in different subgroups of diffuse large B-cell lymphoma. Onkologie 2009; 32: 719-23.

70 Chang JE, Seo S, Kim KM, et al. Rituximab and CHOP chemotherapy plus GM-CSF for previously untreated diffuse large B-cell lymphoma in the elderly: a Wisconsin oncology network study. Clin Lymphoma Myeloma Leuk 20IO; I0: 379-84.

7I Simon A, Peoch M, Casassus P, et al. Upfront VIP-reinforced- ABVD (VIP-rABVD) is not superior to $\mathrm{CHOP} / 2 \mathrm{I}$ in newly diagnosed peripheral $\mathrm{T}$ cell lymphoma. Results of the randomized phase III trial GOELAMS-LTP95. Br J Haematol 20I0; 151: 159-66.

72 Ohmachi K, Tobinai K, Kobayashi Y, et al. Phase III trial of CHOP-2I versus CHOP-I4 for aggressive non-Hodgkin's lymphoma: final results of the Japan Clinical Oncology Group Study, JCOG 9809. Ann Oncol 20II; 22: 1382-9I.

73 Peyrade F, Jardin F, Thieblemont C, et al. Attenuated immunochemotherapy regimen (R-miniCHOP) in elderly patients older than 80 years with diffuse large B-cell lymphoma: a multicentre, single-arm, phase 2 trial. Lancet Oncol 20II; I2: 460-68.

74 Récher C, Coiffier B, Haioun C, et al. Intensified chemotherapy with ACVBP plus rituximab versus standard CHOP plus rituximab for the treatment of diffuse large B-cell lymphoma (LNHo3-2B): an open-label randomised phase 3 trial. Lancet 20II; 378: 1858-67.

75 Vitolo U, Chiappella A, Ferreri AJM, et al. First-line treatment for primary testicular diffuse large B-cell lymphoma with rituximab- CHOP, CNS prophylaxis, and contralateral testis irradiation: final results of an international phase II trial. J Clin Oncol 20II; 29: 2766-72.

76 Watanabe T, Tobinai K, Shibata T, et al. Phase II/III study of R-CHOP-2I versus R-CHOP-I4 for untreated indolent B-cell non-Hodgkin's lymphoma: JCOG 0203 trial. J Clin Oncol 20II; 29: 3990-98. 
77 Kagami Y, Itoh K, Tobinai K, et al. Phase II study of cyclophosphamide, doxorubicin, vincristine, prednisolone (CHOP) therapy for newly diagnosed patients with low- and low-intermediate risk, aggressive non-Hodgkin's lymphoma: final results of the Japan Clinical Oncology Group Study, JCOG9508. Int J Hematol 2012; 96: 74-83.

78 Kluin-Nelemans HC, Hoster E, Hermine O, et al. Treatment of older patients with mantle-cell lymphoma. N Engl J Med 20I2; 367: 520-3I.

79 Shin HJ, Chung JS, Song MK, Kim SK, Choe S, Cho GJ. Addition of rituximab to reduced-dose CHOP chemotherapy is feasible for elderly patients with diffuse large B-cell lymphoma. Cancer Chemother Pharmacol 20I2; 69: II65-72.

80 Cunningham D, Hawkes EA, Jack A, et al. Rituximab plus cyclophosphamide, doxorubicin, vincristine, and prednisolone in patients with newly diagnosed diffuse large B-cell non-Hodgkin lymphoma: a phase 3 comparison of dose intensification with I4-day versus 2I-day cycles. Lancet 20I3; 38I: 18I7-26.

8I Delarue R, Tilly H, Mounier N, et al. Dose-dense rituximab-CHOP compared with standard rituximab-CHOP in elderly patients with diffuse large B-cell lymphoma (the LNHo3-6B study): a randomised phase 3 trial. Lancet Oncol 2013; I4: 525-33.

82 Federico M, Luminari S, Dondi A, et al. R-CVP versus R-CHOP versus R-FM for the initial treatment of patients with advanced-stage follicular lymphoma: results of the FOLLo5 trial conducted by the Fondazione Italiana Linfomi. J Clin Oncol 2013; 31: 1506-13.

83 Herbrecht R, Cernohous P, Engert A, et al. Comparison of pixantrone-based regimen (CPOP-R) with doxorubicin-based therapy (CHOP-R) for treatment of diffuse large B-cell lymphoma. Ann Oncol 2013; 24: 26I8-23.

84 Press OW, Unger JM, Rimsza LM, et al. Phase III randomized intergroup trial of CHOP plus rituximab compared with CHOP chemotherapy plus (III)iodine-tositumomab for previously untreated follicular non-Hodgkin lymphoma: SWOG Soor6. J Clin Oncol 2013; 31: 314-20.

85 Rummel MJ, Niederle N, Maschmeyer G, et al. Bendamustine plus rituximab versus CHOP plus rituximab as first-line treatment for patients with indolent and mantle-cell lymphomas: an openlabel, multicentre, randomised, phase 3 non-inferiority trial. Lancet 2013; 38I: I203-IO.

86 Stiff PJ, Unger JM, Cook JR, et al. Autologous transplantation as consolidation for aggressive nonHodgkin's lymphoma. N Engl J Med 2013; 369: I68I-90.

87 Friedberg JW, Unger JM, Burack WR, et al. R-CHOP with iodine-I3I tositumomab consolidation for advanced stage diffuse large B-cell lymphoma (DLBCL): SWOG So433. Br J Haematol 20I4; I66: 382-89.

88 Held G, Murawski N, Ziepert M, et al. Role of radiotherapy to bulky disease in elderly patients with aggressive B-cell lymphoma. J Clin Oncol 20I4; 32: III2-I8.

89 Pfreundschuh M, Poeschel V, Zeynalova S, et al. Optimization of rituximab for the treatment of diffuse large B-cell lymphoma (II): extended rituximab exposure time in the SMARTE-R-CHOP-I4 trial of the german high-grade non-Hodgkin lymphoma study group. J Clin Oncol 20I4; 32: 4127-33.

90 Seymour JF, Pfreundschuh M, Trněný M, et al. R-CHOP with or without bevacizumab in patients with previously untreated diffuse large B-cell lymphoma: final MAIN study outcomes. Haematologica 20I4; 99: 1343-49.

9I Bozzoli V, Tisi MC, Maiolo E, et al. Four doses of unpegylated versus one dose of pegylated filgrastim as supportive therapy in R-CHOP-I4 for elderly patients with diffuse large B-cell lymphoma. Br J Haematol 2015; 169: 787-94.

92 Cortelazzo S, Tarella C, Gianni AM, et al. Randomized Trial Comparing R-CHOP Versus High-Dose Sequential Chemotherapy in High-Risk Patients With Diffuse Large B-Cell Lymphomas. J Clin Oncol 2016; 34: 4015-22.

93 Fridrik MA, Jaeger U, Petzer A, et al. Cardiotoxicity with rituximab, cyclophosphamide, non-pegylated liposomal doxorubicin, vincristine and prednisolone compared to rituximab, cyclophosphamide, doxorubicin, vincristine, and prednisolone in frontline treatment of patients with diffuse large B-cell lymphoma: A randomised phase-III study from the Austrian Cancer Drug Therapy Working Group [Arbeitsgemeinschaft Medikamentöse Tumortherapie AGMT](NHL-I4). Eur J Cancer 2016; 58: II2-2I. 
94 Hermine O, Hoster E, Walewski J, et al. Addition of high-dose cytarabine to immunochemotherapy before autologous stem-cell transplantation in patients aged 65 years or younger with mantle cell lymphoma (MCL Younger): a randomised, open-label, phase 3 trial of the European Mantle Cell Lymphoma Network. Lancet 2016; 388: 565-75.

95 Nolasco-Medina D, Reynoso-Noveron N, Mohar-Betancourt A, Aviles-Salas A, García-Perez O, Candelaria M. Comparison of three chemotherapy regimens in elderly patients with diffuse large B cell lymphoma: experience at a single national reference center in Mexico. BioMed Res Int 20I6; 20I6: 9817606.

96 Kraeber-Bodere F, Pallardy A, Maisonneuve H, et al. Consolidation anti-CD22 fractionated radioimmunotherapy with ${ }^{90}$ Y-epratuzumab tetraxetan following R-CHOP in elderly patients with diffuse large B-cell lymphoma: a prospective, single group, phase 2 trial. Lancet Haematol 20I7; 4: e35-45.

$97 \mathrm{Hu}$ X, Zeng M, Yang SE, et al. Efficacy of rituximab combined with CHOP for treating patients with diffuse large B-cell lymphoma. Medicine (Baltimore) 20I7; 96: e8494.

98 Li L, Duan W, Zhang L, et al. The efficacy and safety of gemcitabine, cisplatin, prednisone, thalidomide versus $\mathrm{CHOP}$ in patients with newly diagnosed peripheral T-cell lymphoma with analysis of biomarkers. Br J Haematol 20I7; I78: 772-80.

99 Thieblemont C, Tilly H, Gomes da Silva M, et al. Lenalidomide maintenance compared with placebo in responding elderly patients with diffuse large B-cell lymphoma treated with first-line rituximab plus cyclophosphamide, doxorubicin, vincristine, and prednisone. J Clin Oncol 20I7; 35: 2473-8I.

IOo Vitolo U, Trněný M, Belada D, et al. Obinutuzumab or rituximab plus cyclophosphamide, doxorubicin, vincristine, and prednisone in previously untreated diffuse large B-cell lymphoma. J Clin Oncol 20I7; 35: 3529-37.

IOI Yoon DH, Sohn BS, Oh SY, et al. Feasibility of abbreviated cycles of immunochemotherapy for completely resected limited-stage CD20+ diffuse large B-cell lymphoma (CISL I2-09). Oncotarget 20I7; 8: 13367-74.

IO2 Barr PM, Li H, Burack WR, et al. R-CHOP, radioimmunotherapy, and maintenance rituximab in untreated follicular lymphoma (SWOG So8oI): a single-arm, phase 2, multicentre study. Lancet Haematol 20I8; 5: eIO2-08.

I03 Gleeson M, Peckitt C, To YM, et al. CHOP versus GEM-P in previously untreated patients with peripheral T-cell lymphoma (CHEMO-T): a phase 2, multicentre, randomised, open-label trial. Lancet Haematol 20I8; 5: ei9o-200.

IO4 Lamy T, Damaj G, Soubeyran P, et al. R-CHOP I4 with or without radiotherapy in nonbulky limitedstage diffuse large B-cell lymphoma. Blood 20I8; I3I: I74-8I.

IO5 Xu PP, Fu D, Li JY, et al. Anthracycline dose optimisation in patients with diffuse large B-cell lymphoma: a multicentre, phase 3, randomised, controlled trial. Lancet Haematol 20I9; 6: e328-37.

IO6 Jones SE, Miller TP, Connors JM. Long-term follow-up and analysis for prognostic factors for patients with limited-stage diffuse large-cell lymphoma treated with initial chemotherapy with or without adjuvant radiotherapy. J Clin Oncol I989; 7: II86-9I.

I07 Epelbaum R, Haim N, Leviov M, et al. Full dose CHOP chemotherapy in elderly patients with nonHodgkin's lymphoma. Acta Oncol I995; 34: 87-9I.

Io8 Hansen PB, Johnsen HE, Ralfkiaer E, Jensen L, Gaarsdal E, Hansen NE. Short-term rhG-CSF priming before chemotherapy does mobilize blood progenitors but does not prevent chemotherapy induced myelotoxicity: a randomized study of patients with non-Hodgkin's lymphomas. Leuk Lymphoma I995; I9: 453-60.

I09 Reyes F, Lepage E, Ganem G, et al. ACVBP versus CHOP plus radiotherapy for localized aggressive lymphoma. N Engl J Med 2005; 352: 1197-205.

IIo Tobinai K, Ogura M, Itoh K, et al. Randomized phase II study of concurrent and sequential combinations of rituximab plus CHOP (cyclophosphamide, doxorubicin, vincristine and prednisolone) chemotherapy in untreated indolent B-cell non-Hodgkin lymphoma: 7-year follow-up results. Cancer Sci 20IO; IOI: 2579-85. 
III Lahtinen R, Kuikka J, Nousiainen T, Uusitupa M, Länsimies E. Cardiotoxicity of epirubicin and doxorubicin: a double-blind randomized study. Eur J Haematol I99I; 46: 30I-05.

II2 Bezwoda W, Rastogi RB, Erazo Valla A, et al. Long-term results of a multicentre randomised, comparative phase III trial of CHOP versus CNOP regimens in patients with intermediate- and highgrade non-Hodgkin's lymphomas. Eur J Cancer 1995; 3IA: 903-II.

II3 Miller TP, Dahlberg S, Cassady JR, et al. Chemotherapy alone compared with chemotherapy plus radiotherapy for localized intermediate- and high-grade non-Hodgkin's lymphoma. N Engl J Med I998; 339: 2I-26.

II4 Mori M, Kitamura K, Masuda M, et al. Long-term results of a multicenter randomized, comparative trial of modified CHOP versus THP-COP versus THP-COPE regimens in elderly patients with nonHodgkin's lymphoma. Int J Hematol 2005; 8I: 246-54.

II5 Kilickap S, Yavuz B, Aksoy S, et al. Addition of rituximab to chop does not increase the risk of cardiotoxicity in patients with non- Hodgkin's lymphoma. Med Oncol 2008; 25: 437-42.

II6 Meiners B, Shenoy C, Zordoky BN. Clinical and preclinical evidence of sex-related differences in anthracycline-induced cardiotoxicity. Biol Sex Differ 20I8; 9:38.

II7 Linschoten M, Teske AJ, Baas AF, et al. Truncating titin (TTN) variants in chemotherapy-induced cardiomyopathy. J Card Fail 2017; 23: 476-79.

II8 Le Deley MC, Leblanc T, Shamsaldin A, et al. Risk of secondary leukemia after a solid tumor in childhood according to the dose of epipodophyllotoxins and anthracyclines: a case-control study by the Société Française d'Oncologie Pédiatrique. J Clin Oncol 2003; 2I: IO74-8I.

II9 Feijen EAM, Leisenring WM, Stratton KL, et al. Derivation of anthracycline and anthraquinone equivalence ratios to doxorubicin for late-onset cardiotoxicity. JAMA Oncol 2019; 5: 864-7I.

I20 Xue K, Gu JJ, Zhang Q, et al. Cardiotoxicity as indicated by LVEF and troponin T sensitivity following two anthracycline-based regimens in lymphoma: results from a randomized prospective clinical trial. Oncotarget 2016; 7:32519-31.

I2I Swain SM, Whaley FS, Ewer MS. Congestive heart failure in patients treated with doxorubicin: a retrospective analysis of three trials. Cancer 2003; 97: 2869-79.

I22 Russell JS, Colevas AD. Adverse event monitoring in oncology clinical trials. Clin Invest 2013; 3: 1157-65.

I23 Witteles RM, Telli M. Underestimating cardiac toxicity in cancer trials: lessons learned? J Clin Oncol 2012; 30: 1916-I8.

I24 Cardinale D, Colombo A, Bacchiani G, et al. Early detection of anthracycline cardiotoxicity and improvement with heart failure therapy. Circulation 2015; I3I: 198I-88.

I25 Goldberg LR, Jessup M. Stage B heart failure: management of asymptomatic left ventricular systolic dysfunction. Circulation 2006; II3: 285I-60.

I26 Fornaro A, Olivotto I, Rigacci L, et al. Comparison of long-term outcome in anthracycline-related versus idiopathic dilated cardiomyopathy: a single centre experience. Eur J Heart Fail 20I8; 20: 898906.

I27 Cardinale D, Colombo A, Lamantia G, et al. Anthracycline-induced cardiomyopathy: clinical relevance and response to pharmacologic therapy. J Am Coll Cardiol 20I0; 55: 213-20.

I28 Ponikowski P, Voors AA, Anker SD, et al. 20I6 ESC Guidelines for the diagnosis and treatment of acute and chronic heart failure: The Task Force for the diagnosis and treatment of acute and chronic heart failure of the European Society of Cardiology (ESC) developed with the special contribution of the Heart Failure Association (HFA) of the ESC. Eur Heart J 2016; 37: 2129-200.

I29 Riley RD, Lambert PC, Abo-Zaid G. Multivariate meta-analysis using individual participant data. BMJ 2OIO; 340: c22I.

I30 Tudur Smith C, Marcucci M, Nolan SJ, et al. Individual participant data meta-analyses compared with meta-analyses based on aggregate data. Cochrane Database Syst Rev 2016; 9: MRooooo7.

I3I Lancellotti P, Suter TM, López-Fernández T, et al. Cardio-Oncology Services: rationale, organization, and implementation. Eur Heart J 2019; 40: 1756-63.

I32 Teske AJ, Linschoten M, Kamphuis JAM, et al. Cardio-oncology: an overview on outpatient management and future developments. Neth Heart J 20I8; 26: 52I-32. 
I33 Plana JC, Galderisi M, Barac A, et al. Expert consensus for multimodality imaging evaluation of adult patients during and after cancer therapy: a report from the American Society of Echocardiography and the European Association of Cardiovascular Imaging. Eur Heart J Cardiovasc Imaging 20I4; I5: I063-93.

I34 Oikonomou EK, Kokkinidis DG, Kampaktsis PN, et al. Assessment of prognostic value of left ventricular global longitudinal strain for early prediction of chemotherapy-induced cardiotoxicity: a systematic review and meta-analysis. JAMA Cardiol 2019; 4: I007.

I35 Michel L, Mincu RI, Mahabadi AA, et al. Troponins and brain natriuretic peptides for the prediction of cardiotoxicity in cancer patients: a meta-analysis. Eur J Heart Fail 20I9; published online Nov I2. DOI:I0.I002/ejhf.I63I.

I36 Avila MS, Ayub-Ferreira SM, de Barros Wanderley MR Jr, et al. Carvedilol for Prevention of Chemotherapy-Related Cardiotoxicity: The CECCY Trial. J Am Coll Cardiol 20I8; 7I: 228I-90.

I37 Gulati G, Heck SL, Ree AH, et al. Prevention of cardiac dysfunction during adjuvant breast cancer therapy (PRADA): a $2 \times 2$ factorial, randomized, placebo-controlled, double-blind clinical trial of candesartan and metoprolol. Eur Heart J 20I6; 37: I67I-80.

I38 van Dalen EC, Caron HN, Dickinson HO, Kremer LC. Cardioprotective interventions for cancer patients receiving anthracyclines. Cochrane Database Syst Rev 20II; 6: CDoo39I7.

I39 Trotti A, Pajak TF, Gwede CK, et al. TAME: development of a new method for summarising adverse events of cancer treatment by the Radiation Therapy Oncology Group. Lancet Oncol 2007; 8: 6I3-24. 


\section{Supplemental File I Search string}

\section{Pubmed}

\begin{tabular}{|c|c|}
\hline Domain & $\begin{array}{l}\text { "Lymphoma"[Mesh] OR "Lymphoma, Non-Hodgkin"[Mesh] OR "Lymphoma, T-Cel- } \\
\text { l"[Mesh] OR "Lymphoma, B-Cell"[Mesh] OR "Lymphoma, Mantle-Cell”[Mesh] OR } \\
\text { "Lymphoma, Large B-Cell, Diffuse"[Mesh] OR "Lymphoma, Follicular"[Mesh] OR "Bur- } \\
\text { kitt Lymphoma"[Mesh] OR "lymphoma" OR "Non-Hodgkin lymphoma" OR "T-Cell } \\
\text { Lymphoma" OR "B-Cell Lymphoma" OR "Mantle-Cell lymphoma" OR "Diffuse Large } \\
\text { B-Cell lymphoma" OR "DLBCL" OR "Follicular Lymphoma" OR "Burkitt Lymphoma" }\end{array}$ \\
\hline Determinant & $\begin{array}{l}\text { "R-CHOP”[tiab] OR “CHOP”[tiab] OR "R-CHOP protocol"[Supplementary Concept] OR } \\
\text { "CHOP protocol" [Supplementary Concept] }\end{array}$ \\
\hline Specification & Clinical Trial[ptyp] \\
\hline
\end{tabular}

\section{Cochrane Central Register of Controlled Trials}

Search Term CHOP OR R-CHOP

\section{Embase}

\begin{tabular}{ll}
\hline \hline Domain & $\begin{array}{l}\text { \#I 'hematologic disease' OR 'leukemia' OR 'lymphoma' OR 'nonhodgkin lymphoma' } \\
\text { OR 'chronic lymphatic leukemia' AND [embase]/lim }\end{array}$ \\
\hline \hline Determinant & $\begin{array}{l}\text { \#2 'cyclophosphamide plus doxorubicin plus prednisolone plus rituximab plus vincris- } \\
\text { tine'/exp OR 'chop protocol'/exp AND [embase]/lim }\end{array}$ \\
\hline \hline Specification & clinical study' \\
\hline \hline
\end{tabular}

\section{Supplemental File 2 Results of the meta-analyses}

\begin{tabular}{|c|c|c|c|}
\hline Outcome & $\begin{array}{l}\text { Fixed-effects model } \\
{[95 \% \mathrm{CI}]}\end{array}$ & $\begin{array}{l}\text { Random-effects model } \\
{[95 \% \mathrm{CI}]}\end{array}$ & Heterogeneity \\
\hline $\begin{array}{c}\text { Grade } 3+4 \text { CVAE } \\
(n=96)\end{array}$ & $\begin{array}{c}2.46 \% \\
{[2.19 ; 2.75]}\end{array}$ & $\begin{array}{c}2.35 \% \\
{[\text { I.8I; } 2.93]}\end{array}$ & $\begin{array}{c}\tau^{2}=0.0042 ; \mathrm{I}^{2}=7 \mathrm{I} .4 \% \\
Q=326.2 \mathrm{I}, \mathrm{p}<0.000 \mathrm{I}\end{array}$ \\
\hline $\begin{array}{l}\text { Overall heart failure } \\
(\mathrm{n}=47)\end{array}$ & $\begin{array}{c}3.00 \% \\
{[2.53 ; 3.50]} \\
\end{array}$ & $\begin{array}{c}4.62 \% \\
{[2.25 ; 7.65]} \\
\end{array}$ & $\begin{array}{c}\tau^{2}=0.0384 ; I^{2}=95.05 \% \\
Q=527.33, p<0.000 I\end{array}$ \\
\hline $\begin{array}{l}\text { Grade } \mathrm{I}+2 \text { CVAE } \\
(\mathrm{n}=30)\end{array}$ & $\begin{array}{c}7.54 \% \\
{[6.77 \%-8.35 \%]}\end{array}$ & $\begin{array}{c}8.52 \% \\
{[5.58 ; \text { II.96] }}\end{array}$ & $\begin{array}{c}\tau^{2}=0.0209 ; \mathrm{I}^{2}=92.72 \% \\
Q=356.44, \mathrm{p}<0.000 \mathrm{I}\end{array}$ \\
\hline $\begin{array}{l}\text { Treatment discontinuation } \\
\qquad(\mathrm{n}=56)\end{array}$ & $\begin{array}{c}<0.0001 \% \\
{[0 ; 0.05]}\end{array}$ & $\begin{array}{c}<0.0001 \% \\
{[0 ; 0.05]}\end{array}$ & $\begin{array}{c}\tau^{2}=0 ; I^{2}=0 \% \\
Q=40.87, p=0.92\end{array}$ \\
\hline $\begin{array}{l}\text { Treatment-related cardiac death } \\
\qquad(\mathrm{n}=\mathrm{I} 34)\end{array}$ & $\begin{array}{c}0.03 \% \\
{[0 ; 0.10]}\end{array}$ & $\begin{array}{c}0.03 \% \\
{[0 ; 0.10]}\end{array}$ & $\begin{array}{c}\tau^{2}=0 ; I^{2}=0 \% \\
Q=\mathrm{I} 32.63, p=0.49\end{array}$ \\
\hline
\end{tabular}

CVAE cardiovascular adverse events; CI confidence interval 
Supplemental File 3 Study characteristics of studies who reported on the outcome 'heart failure' and on the performance of cardiac screening after therapy (A); Pooled proportion of overall heart failure in study who did- and did not handle in- and exclusion criteria regarding cardiac function (B).

A - Study characteristics of studies with and without cardiac screening after therapy

\begin{tabular}{cccc}
\hline Variable & $\begin{array}{c}\text { No screening after } \\
\text { therapy }(\mathbf{n}=30)\end{array}$ & $\begin{array}{c}\text { Screening after therapy } \\
(\mathbf{n}=\mathbf{I} 4)\end{array}$ & p-value \\
\hline Age (years) & $6 \mathrm{I} .7 \pm 8.2$ & $58.8 \pm 8.5$ & $0.3 \mathrm{I}$ \\
\hline \hline Gender (\% of females) & $43 . \mathrm{I} \pm \mathrm{I} 0.4$ & $46.0 \pm 4.7$ & 0.24 \\
\hline Number of cycles & $6 . \mathrm{I} \pm \mathrm{I} .3$ & $6.2 \pm \mathrm{I} .6$ & 0.86 \\
\hline \hline Interval between cycles (days) & $\mathrm{I} 8.8 \pm 3.4$ & $2 \mathrm{I} .2 \pm 2.9$ & $0.30^{*}$ \\
\hline Doxorubicin dose $\left(\mathrm{mg} / \mathrm{m}^{2}\right)$ & $50.0 \pm 0.0$ & $48.5 \pm 5.5$ & 0.34 \\
\hline \hline
\end{tabular}

* p-value <0.05; Values expressed as mean \pm standard deviation.

B - Pooled proportion of overall heart failure of studies with and without in- and exclusion criteria regarding cardiac function

\begin{tabular}{cccc}
\hline & $\begin{array}{c}\text { No criteria regarding } \\
\text { cardiac function }(\mathbf{n}=\mathbf{2 0})\end{array}$ & $\begin{array}{c}\text { Criteria regarding car- } \\
\text { diac function }(\mathbf{n = 2 7})\end{array}$ & p-value \\
\hline Overall heart failure & $2.8 \mathrm{I} \%$ [0.60-6.I5] & $6.0 \mathrm{I} \%$ [2.37-I0.9I] & $0.2 \mathrm{I}$ \\
\hline \hline
\end{tabular}

* p-value <0.05; Values expressed as pooled proportion with [95\% confidence interval]. 


\section{Cardiovascular adverse events following treatment for non-Hodgkin lymphoma Authors' reply}

Lancet Haematology 2020; 7(8):e557-e558

Reply to: Jayaraj R, Kumarasamy C, Raymond G, Shaw P. Cardiovascular adverse events following treatment for non-Hodgkin lymphoma. Lancet Haematology 2020;7(8):e557

M Linschoten, JAM Kamphuis, FW Asselbergs

It is widely accepted that heart failure is a progressive disease, and overt heart failure can be preceded by a phase of asymptomatic left ventricular systolic dysfunction. A meta-analysis of II studies of patients with asymptomatic left ventricular systolic dysfunction ( $n=25369$ ) followed on average for $7 \cdot 9$ years, found that the relative risk of developing heart failure was $4 \cdot 6(95 \% \mathrm{CI} 2 \cdot 2-9 \cdot 8)[\mathrm{I}]$. Therefore, efforts to mitigate the progression to advanced heart failure stages is of pivotal importance. On the basis of a number of randomised controlled trials, the European Society of Cardiology [2] and the American College of Cardiology Foundation and American Heart Association [3] issued a Class I recommendation for the initiation of angiotensin-converting enzyme inhibitors in patients with asymptomatic left ventricular systolic dysfunction, regardless of the underlying cause. We therefore stand behind our statement to treat asymptomatic left ventricular systolic dysfunction after anthracycline-containing chemotherapy aiming to delay the onset of heart failure, in line with these guidelines.

We have re-evaluated the three studies [4-6] in the meta-analysis of cardiac screening after therapy, to identify possible explanations for the higher incidence of overall heart failure. In the smallest of these studies ( $n=12)$ [4], patients received higher doses of doxorubicin, providing a plausible explanation for the higher incidence. However, for the other two studies [5-6] we did not find any differences in patient characteristics, definitions of cardiac dysfunction, or handled cardiac imaging techniques that could explain the observed differences. A sensitivity analysis excluding these studies separately, one at a time, did not change the pooled proportion of patients with treatment-related overall heart failure considerably (8.I-IO $6 \%$ ), nor did it change the statistical significance. Our results are further supported by a landmark study by Cardinale and colleagues [7] among 2625 
patients treated with similar cumulative doxorubicin doses. In this study, left ventricular dysfunction was detected in $9 \%$ of patients on average 3.5 months (IQR I-6) after chemotherapy when a serial echocardiographic assessment was done. In a report pooling the results of three phase 3 trials in patients with breast cancer $(n=630)$, left ventricular dysfunction was diagnosed in $9 \%$ of patients at a cumulative doxorubicin dose of $250 \mathrm{mg} / \mathrm{m}^{2}$, and $18 \%$ of patients at a cumulative doxorubicin dose of $350 \mathrm{mg} / \mathrm{m}^{2}$ [8] In light of these results, despite the heterogeneity in the reported incidence of overall heart failure among studies that screened cardiac function post-chemotherapy, we are confident that there is a clear under-reporting of this treatment-related side effect in patients with non-Hodgkin's lymphoma treated with cyclophosphamide, doxorubicin, vincristine, and prednisone with rituximab, especially in the absence of cardiac monitoring.

\section{References}

I Echouffo-Tcheugui JB, Erqou S, Butler J, Yancy CW, Fonarow GC. Assessing the risk of progression from asymptomatic left ventricular dysfunction to overt heart failure: a systematic overview and meta-analysis. JACC Heart Fail 20I6; 4: 237-48.

2 Ponikowski P, Voors AA, Anker SD, et al.20I6 ESC Guidelines for the diagnosis and treatment of acute and chronic heart failure: the task force for the diagnosis and treatment of acute and chronic heart failure of the European Society of Cardiology (ESC) Developed with the special contribution of the Heart Failure Association (HFA) of the ESC. Eur Heart J 2016; 37: 2129-200.

3 Yancy CW, Jessup M, Bozkurt B, et al. 2013 ACCF/AHA guideline for the management of heart failure: a report of the American College of Cardiology Foundation/ American Heart Association Task Force on practice guidelines. J Am Coll Cardiol 2013;62: eI47-239.

4 Lahtinen R, Kuikka J, Nousiainen T, Uusitupa M, Länsimies E. Cardiotoxicity of epirubicin and doxorubicin: a double-blind randomized study. Eur J Haematol I99I; 46: 30I-05.

5 Herbrecht R, Cernohous P, Engert A, et al. Comparison of pixantrone-based regimen (CPOP-R) with doxorubicin-based therapy (CHOP-R) for treatment of diffuse large B-cell lymphoma. Ann Oncol 2013; 24: 26I8-23.

6 Fridrik MA, Jaeger U, Petzer A, et al. Cardiotoxicity with rituximab, cyclophosphamide, non-pegylated liposomal doxorubicin, vincristine and prednisolone compared to rituximab, cyclophosphamide, doxorubicin, vincristine, and prednisolone in frontline treatment of patients with diffuse large B-cell lymphoma: A randomised phase-III study from the Austrian Cancer Drug Therapy Working Group [Arbeitsgemeinschaft Medikamentöse Tumortherapie AGMT] (NHL-I4). Eur J Cancer 20I6; 58: II2-2I.

7 Cardinale D, Colombo A, Bacchiani G, et al. Early detection of anthracycline cardiotoxicity and improvement with heart failure therapy. Circulation 20I5; I3I: 198I-88.

8 Swain SM, Whaley FS, Ewer MS. Congestive heart failure in patients treated with doxorubicin: a retrospective analysis of three trials. Cancer 2003; 97: 2869-79. 
Chapter 5

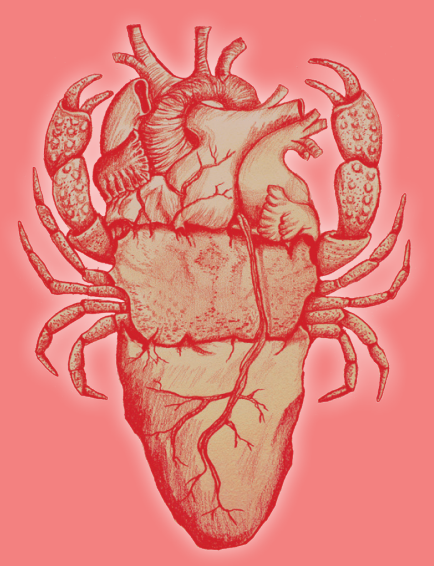




\section{Early- and late diagnosed anthracycline-induced cardiac dysfunction: echocardiographic characterization and response to heart failure therapy}

Accepted for publication in Cardio-Oncology

JAM Kamphuis' ${ }^{1}$, M Linschoten ${ }^{\mathrm{1}}$, MJ Cramer ${ }^{\mathrm{I}}$, PA Doevendans ${ }^{1,2,3}$, FW Asselbergs ${ }^{1,4,5}$, AJ Teske ${ }^{1}$

I Department of Cardiology, Division of Heart and Lungs, University Medical Centre Utrecht, Utrecht, The Netherlands 2 Netherlands Heart Institute, Utrecht, The Netherlands

3 Central Military Hospital, Utrecht, The Netherlands

4 Health Data Research UK and Institute of Health Informatics, University College London, London, UK

5 Institute of Cardiovascular Science, Faculty of Population Health Sciences, University College London, London, UK 


\begin{abstract}
Aims Anthracycline-induced cardiac dysfunction (AICD) has been described as a phenomenon of a continuous progressive decline of cardiac function, leading to dilated cardiomyopathy (DCM). This progressive nature suggests that patients with a delayed AICD diagnosis have greater compromise of cardiac function, more adverse remodeling and poor response to heart failure (HF) treatment. This study delineates the impact of timing of AICD diagnosis on echocardiographic characteristics and response to HF treatment.
\end{abstract}

Methods and Results At our cardio-oncology outpatient clinic, 92 patients (age 5I.6 \pm I6.2 years, anthracycline dose $329[200-329] \mathrm{mg} / \mathrm{m} 2)$ were diagnosed early $(<\mathrm{I}$ year) and late (>I year) after 4.0 vs. 47.7 months after anthracycline therapy respectively. No echocardiographic differences were found between patients diagnosed early vs. late (LVEF $43.6 \pm 4.9 \%$ vs. $43.0 \pm 6.2 \%$ and iEDV 63.6 vs. $62.9 \mathrm{~mL} / \mathrm{m} 2$ ). Eighty-three percent of patients presented with mild LV dysfunction and in $79 \%$ the $\mathrm{LV}$ was not dilated. Patients diagnosed early were more likely to have (partial) recovery of cardiac function upon HF treatment initiation ( $\mathrm{p}=\mathrm{o} .0 \mathrm{II})$.

Conclusions Patients with AICD presented with a hypokinetic non-dilated cardiomyopathy, rather than typical DCM. Though timing of AICD diagnosis did not impact HF disease severity, patients diagnosed early were more likely to recover upon HF treatment. 


\section{Introduction}

Anthracyclines are potent antineoplastic drugs that constitute a cornerstone in the treatment of sarcomas, breast cancer and haematological malignancies. Shortly after the introduction of these agents in the I960s, cardiac dysfunction was discovered to be an important dose-limiting side effect [I]. However, despite dosage restrictions, the incidence of anthracycline-induced cardiac dysfunction (AICD) is currently still 9-I8\% [2,3].The prognosis of AICD is poor, with cardiovascular mortality rates ranging from $9 \%$ at 5 - and $24 \%$ at IO-years [4], up to more dramatic outcomes of $60 \%$ at 2-years in patients that have developed symptomatic heart failure (HF) [5].

Anthracyclines induce irreversible cardiomyocyte damage, reflected by a troponin release during-, or early after anthracycline infusion [6,7]. The initial damage, resulting in a declined pumping capacity, will not coincide with the development of clinical manifestations of $\mathrm{HF}$ in a majority of patients. However, if serial echocardiographic assessment is performed, almost all asymptomatic declines are detected within the first year after anthracycline-containing chemotherapy [3]. The delayed onset of symptomatic HF may be related to the activation of compensatory mechanisms that modulate left ventricular (LV) function within a range, such that the functional capacity is preserved or only minimally depressed [8]. These compensatory mechanisms include the activation of neurohumoral systems and adaptive changes within the myocardium commonly referred to as LV remodeling. However, prolonged activation of these compensatory mechanisms ultimately has detrimental effects on the heart, leading to adverse LV remodeling with dilatation and wall thinning [9]. The onset of these changes typically marks the transition from asymptomatic- to symptomatic HF. Based on this theory, it can be postulated that over time, patients with AICD develop progressive systolic dysfunction with dilated compartments, as is the case in patients with familial dilated cardiomyopathy (DCM), long lasting left-sided valvular disease or hypertension.

The aim of this consecutive cohort study was to (I) evaluate the impact of a delayed diagnosis (>I year after anthracycline containing treatment) on echocardiographic characteristics and (2) assess the influence of timing of diagnosis on HF treatment response.

\section{Methods}

\section{Study population}

In April 20I5, a cardio-oncology clinic was launched at the University Medical Center Utrecht, the Netherlands, of which the protocol has been described in detail previously [Io]. In short, the patient population mainly consists of patients with breast cancer or hematological malignancies who are at increased risk for AICD due to treatment- or patient-related factors. Furthermore, cancer patients and survivors that have not received cardiac surveillance in the past (e.g. received potentially cardiotoxic treatment prior to the initiation of the clinic in 20I5), are referred for screening of long-term cardiovascular complications at the discretion of the treating (hemato-)oncologist. One of the primary aims of this clinic is to facilitate a timely diagnosis of cancer therapy-related cardiac dysfunction 
(CTRCD) by performing serial echocardiographic assessments. When patients are diagnosed with CTRCD, guideline-based HF therapy [II] is initiated if there are signs or symptoms of $\mathrm{HF}$, or when the LV ejection fraction (LVEF) reaches $<45 \%$ regardless of the presence of cardiac complaints [Io].

All patients referred to the cardio-oncology clinic between April 2015 and February 2019 who were treated with anthracyclines were identified. Subsequently, we selected patients that showed impaired LV function on echocardiography or cardiac magnetic resonance (CMR). We excluded patients with LV dysfunction on multiple-gated acquisition (MUGA) scans, with echocardiographic images of insufficient quality, and patient that had received treatment with the cardiotoxic monoclonal antibody trastuzumab. Time between the initiation of anthracycline-containing therapy and AICD diagnosis was used to divide patients in two groups, namely early- (<I year) and late ( $>$ I year) AICD diagnosis, as described in the ESC Position Paper on Cancer Treatment and Cardiovascular Toxicity [I2]. The study was exempted from formal approval by the Medical Ethics Committee.

\section{Definition of AICD}

Patients were diagnosed with AICD if they met one of the following two criteria: (I) LVEF decline of $\geq$ IO percentage points below the lower limit of normal (LLN) ( $<53 \%)$ from baseline according to the European Association of Cardiovascular Imaging (EACVI)[13], or (2) an LVEF $<50 \%$ measured at $>$ I time-point in case a baseline LVEF measurement prior to anthracycline treatment was not available.

Other possible causes of LV dysfunction were evaluated to determine the likelihood of AICD. Based on this assessment, patients were subdivided into three groups namely, 'definite AICD', 'AICD with concomitant heart disease' and 'possible AICD'. In patients with definite AICD, alternative causes of LV dysfunction were ruled out or deemed highly unlikely. For patients that did not undergo ischemia detection, ischemic heart disease was determined unlikely in the absence of chest pain, low cardiovascular risk profile ( $<2$ risk factors), no coronary artery disease (CAD) on computed tomography (CT) of the chest and lack of regional wall motion abnormalities on echocardiography. Patients were diagnosed with AICD and concomitant heart disease if other causes affected LV function, but these abnormalities were determined to be insufficient to explain the degree of LV dysfunction as a whole. Lastly, in patients with 'possible AICD', severe valvular heart disease, left bundle branch block (LBBB), sepsis-induced cardiac dysfunction and tachycardiomyopathy were ruled out. However, in these patients, the presence of ischemic heart disease had not formally been excluded while there were signs of CAD on the chest CT. None of these patients had reported any chest complaints. The diagnosis of AICD was verified by two authors (JK and ML).

\section{Oncological treatment}

We determined the timing of first anthracycline dose, as well as the total cumulative dose $\left(\mathrm{mg} / \mathrm{m}^{2}\right)$ which was calculated to an equivalent doxorubicin dose [I4]. Moreover, data on mediastinal- or left-sided radiotherapy was collected. 


\section{Echocardiographic analysis}

Echocardiographs were performed by trained technicians at our cardio-oncology program. An extensive analysis was performed on the echocardiogram on which AICD had been diagnosed and on the most recent echocardiogram. All measurements were analyzed by JK, and verified by AT. Reference values and international echocardiography guidelines for echocardiographic examination can be found in Supplemental Table I. LV volumes and -ejection fractions were preferably determined on $3 \mathrm{D}$ echocardiographic images. If $3 \mathrm{D}$ images were not available, 2D biplane (modified Simpson's) algorithm was performed on the 4- and 2-chamber apical view. Global longitudinal strain (GLS) measurements were performed with the vendor's software package using the 4-, 3-, and 2-chamber apical view.

\section{Follow-up}

Patients with a follow-up of $\geq 6$ months, or patients with complete recovery of the LVEF within 6 months, were included in the analysis of AICD reversibility. Reversibility was based on the change between the nadir- and last LVEF measurement and was classified according to the EACVI Expert Consensus [I3]. In patients lacking a baseline LVEF measurements $(n=74)$, we deemed AICD to be $\mathrm{I})$ reversible in case there was an LVEF improvement of $\geq$ IO percentage points to above the LLN, 2) partially reversible if LVEF improved 5-IO percentage points to above the LLN, and 3) irreversible if the LVEF improved <IO percentage points from the nadir and remained below the LLN.

\section{Statistical analysis}

Continuous data are expressed as means and standard deviations (SD) or medians and interquartile ranges [IQR]. Categorical variables are expressed as numbers (percentages). Continuous data were compared using the independent Student's t-test or Mann-Whitney U. Categorical data were tested using Chi-square or Fisher exact test as appropriate. Correlation was calculated with either Pearson or Spearman correlation, where appropriate. Differences between >2 groups were calculated using one-way analysis of variance (ANOVA) with Bonferroni post correction for multiple comparisons or the Kruskal-Wallis test. A two-sided p-value $<0.05$ was considered significant. Statistical analyses were performed using SPSS Statistics, version 25 (IBM Corp., Armonk, NY, USA).

\section{Results}

\section{Study population}

Between April 2015 to February 2019, a total of 512 patients had been evaluated at the cardio-oncology outpatient clinic (Figure I). Anthracyclines were administered in 342 patients, of which 44 patients were not eligible for this study due to concomitant treatment with trastuzumab. Of the remaining 298 patients, IO7 (35.9\%) had LV dysfunction. In 6 patients, the underlying cause for the impaired LV dysfunction was deemed not related to anthracyclines. Additionally, nine patients had echocardiographic images of insufficient quality to perform reliable measurements. In total, 92 patients were included in this study. 


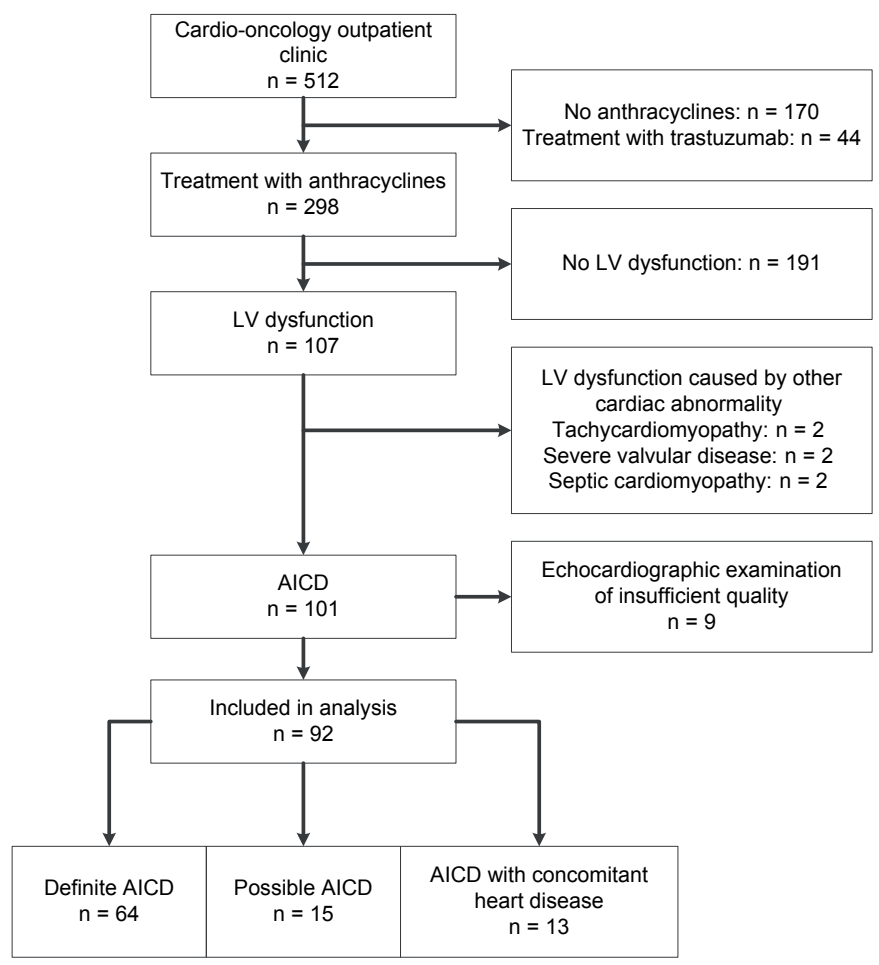

Figure I Flow chart of the study population selection

All patient characteristics at time of AICD diagnosis are outlined in Table I. Most patients were referred for screening of cardiovascular toxicity $(n=78 ; 85 \%)$ and cardiac screening prior to stem cell transplantation was the main reason for referral $(n=56 ; 61 \%)$. Cardiac complaints, including dyspnea, angina and palpitations were the reason for referral in 14 patients (early $n=5$; late $n=9$ ).

\section{Anthracycline-induced cardiac dysfunction}

Sixty-four patients (70\%) were diagnosed with definite AICD, and thirteen patients (I4\%) had AICD with concomitant heart disease(s), including LBBB $(n=3), \operatorname{CAD}(n=5)$, moderate mitral valve regurgitation $(n=7)$, non-compaction cardiomyopathy $(n=2)$ and hypertrophic cardiomyopathy $(\mathrm{n}=\mathrm{I})$. In $\mathrm{I} 5$ patients ( $\mathrm{I} 6 \%)$, CAD could not be excluded as additional investigations of coronary artery status had not (yet) been performed. These patients were classified as having possible AICD.

Of all 92 patients, 32 (35\%) did not have any known cardiovascular risk factors, $43(47 \%)$ had I risk factor, $\mathrm{I} 7$ ( $19 \%)$ had $\geq 2$ risk factors. Forty-nine patients were diagnosed within I year after anthracycline-initiation (median 4 [I.9-6.4] months). AICD was diagnosed late in 43 patients (median 47.7 [41.7-87.3] months) of which 29 patients were diagnosed $>2$ years after initiation of anthracycline-containing anticancer treatment. All patients had 
Table I Characteristics of study participants

\begin{tabular}{|c|c|c|c|c|}
\hline Demographics & Overall $(\mathrm{n}=92)$ & Early $(n=49)$ & Late $(n=43)$ & p-value \\
\hline Male & $68(74)$ & $38(78)$ & $30(70)$ & \\
\hline Body Mass Index $\left(\mathrm{kg} / \mathrm{m}^{2}\right)$ & $24.5(4.5)$ & $24.8(4.5$ & 24.I ( 4.5 & 0.422 \\
\hline Body Surface Area $\left(\mathrm{m}^{2}\right)$ & I.9 (0.2) & I.9 (0.2) & I.9 (0.2) & 0.536 \\
\hline Age at start cancer therapy (years) & 48.2 (I8.I) & $52.2(\mathrm{I} 5.8)$ & $43.7(19.2)$ & 0.024 \\
\hline Age at diagnosis AICD (years) & 5I.6 (I6.2) & 52.4 (I6.I) & $50.8(\mathrm{I} 6.2)$ & 0.646 \\
\hline \multicolumn{5}{|l|}{ Malignancy } \\
\hline $\begin{array}{l}\text { Hematological } \\
\text { Acute leukemia } \\
\text { Non-Hodgkin lymphoma } \\
\text { Hodgkin lymphoma } \\
\text { Other }\end{array}$ & $\begin{array}{c}86(94) \\
44(5 \mathrm{I}) \\
6(7) \\
26(30) \\
\text { IO (12) }\end{array}$ & $\begin{array}{l}48(98) \\
32(67) \\
\text { I (2) } \\
8(\mathrm{I} 7) \\
7(\mathrm{I} 5)\end{array}$ & $\begin{array}{l}38(88) \\
\mathrm{I} 2(32) \\
5(\mathrm{I} 3) \\
\mathrm{I} 8(47) \\
3(8)\end{array}$ & \\
\hline Breast cancer & $2(2)$ & $\mathrm{o}(\mathrm{o})$ & $2(5)$ & \\
\hline Other solid tumors & $4(4)$ & I ( $(2)$ & $3(7)$ & \\
\hline Cumulative anthracycline dose $\left(\mathrm{mg} / \mathrm{m}^{2}\right)$ & $329[200-329]$ & 329 [180-329] & $308[200-400]$ & o.I14 \\
\hline Chest radiation & $7(8)$ & I ( $(2)$ & $6(\mathrm{I} 4)$ & \\
\hline \multicolumn{5}{|l|}{ Function class at diagnosis } \\
\hline NYHA class I + II & $86(94)$ & $47(96)$ & $39(9 \mathrm{I})$ & \\
\hline NYHA class III + IV & $6(6)$ & $2(4)$ & $4(9)$ & \\
\hline \multicolumn{5}{|l|}{ Electrocardiogram } \\
\hline $\begin{array}{l}\text { Heart rhythm } \\
\text { Sinsur rhythm } \\
\text { Atrial fibrillation }\end{array}$ & $\begin{array}{c}90(98) \\
2(2) \\
\end{array}$ & $\begin{array}{c}48(98) \\
\mathrm{I}(2) \\
\end{array}$ & $\begin{array}{c}42(98) \\
\mathrm{I}(2) \\
\end{array}$ & \\
\hline $\begin{array}{l}\text { Heart axis } \\
\text { Normal } \\
\text { Left } \\
\text { Right }\end{array}$ & $\begin{array}{l}82(89) \\
8(9) \\
2(2) \\
\end{array}$ & $\begin{array}{l}42(86) \\
5(\mathrm{IO}) \\
2(4) \\
\end{array}$ & $\begin{array}{l}40(93) \\
3(7) \\
0(0)\end{array}$ & \\
\hline $\begin{array}{l}\text { Ventricular conduction } \\
\text { Normal } \\
\text { Left bundle brach block } \\
\text { Right bundle branch block }\end{array}$ & $\begin{array}{l}86(93) \\
4(4) \\
2(2)\end{array}$ & $\begin{array}{l}45(92) \\
2(4) \\
2(4)\end{array}$ & $\begin{array}{l}4 \mathrm{I}(95) \\
2(5) \\
\mathrm{o}(\mathrm{o})\end{array}$ & \\
\hline \multicolumn{5}{|l|}{ Cardiovascular risk factors } \\
\hline Hypertension & I9 (2I) & 7 (I4) & I2 (28) & \\
\hline Diabetes Mellitus & 9 (I0) & $3(6)$ & $6(\mathrm{I} 4)$ & \\
\hline Hyperlipidemia & $\mathrm{I} 2(\mathrm{I} 7)$ & $5(\mathrm{I} 3)$ & 7 (19) & \\
\hline $\begin{array}{l}\text { Smoking status } \\
\text { Former smoker } \\
\text { Current smoker } \\
\text { Never smoked }\end{array}$ & $\begin{array}{l}\mathrm{n}=80 \\
3 \mathrm{I}(39) \\
\mathrm{I} 3(\mathrm{I} 6) \\
36(45)\end{array}$ & $\begin{array}{c}\mathrm{n}=42 \\
24(57) \\
6(\mathrm{I} 4) \\
\mathrm{I} 2(29)\end{array}$ & $\begin{array}{l}\mathrm{n}=38 \\
7(\mathrm{I} 8) \\
7(\mathrm{I} 8) \\
24(64)\end{array}$ & \\
\hline Coronary- or peripheral artery disease & I3 (I4) & $8(\mathrm{I} 7)$ & $5(\mathrm{I} 2)$ & \\
\hline \multicolumn{5}{|c|}{ 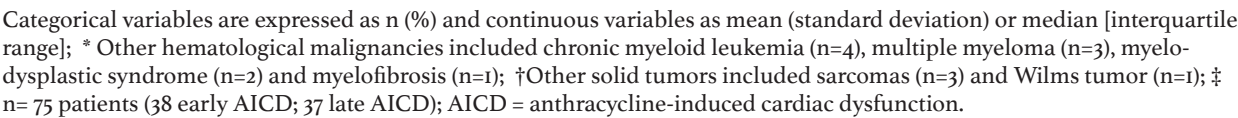 } \\
\hline
\end{tabular}


finished anthracycline-containing therapy and did not receive further treatment with cardiotoxic agents.

\section{Echocardiographic characterization of AICD at diagnosis}

Overall, the LVEF impairment was mild in 76 patients (83\%), moderate in 12 (13\%) and severe in 4 patients $(4 \%)$. No echocardiographic differences were found between early- and late diagnosed AICD (Table 2, Figure 2a). There was no relation between timing of AICD diagnosis and $-\operatorname{LVEF}(\rho=-0.029 p=0.782$; Figure $3 a),-G L S(\rho=-0.099, p=0.374$; Figure $3 b)$, or $\operatorname{iEDV}(\rho=-0.112 ; p=0.288$; Figure $3 c)$ at diagnosis. Seventy-three patients $(79 \%)$ had a normal indexed end-diastolic volume (iEDV) (Figure 3c). We found a decreased, normal, and increased LV mass in 2, 74 and I5 patients, respectively. Out of the I5 patients with increased LV mass, 7 patients had hypertension and 3 patients had moderate mitral valve regurgitation. No relation between timing of AICD diagnosis and LV geometry was established $(\mathrm{p}=0.7 \mathrm{IO})$ (Figure 3d). There was no correlation between cumulative anthracycline dose and LV mass ( $\mathrm{r}=0.02 \mathrm{I}, \mathrm{p}=0.845)$. Thirty-four patients $(38 \%)$ had normal diastolic function, 47 (53\%) had grade I- and 8 (9\%) had grade II diastolic dysfunction.

\section{AICD treatment and clinical follow-up}

The median follow-up time after diagnosis of AICD was 25.0 [10.6-38.2] months (Figure 2b). HF medication was initiated in 68 patients (74\%) (Table 3). Five of these patients were already on ACE-inhibitors or beta-blockers because of hypertension, and additional HF therapy was given. Intolerance to ACE-inhibitors and betablockers was common, and overall the maximum tolerated dose was low (Table 3). Twenty-four patients (26\%) did not receive HF medication since they had an LVEF of $>45 \%$ and lacked cardiac complaints [Io]. Three patients were diagnosed with AICD when they were hospitalized due to HF and 5 patients were hospitalized with decompensated HF after AICD was diagnosed. No patient was in need for cardiac mechanical support or heart transplantation during follow-up. Implantable cardioverter defibrillator (ICD) implantation was performed in 4 patients, of which 3 patients also received cardiac resynchronization therapy. One patient was successfully treated with a MitraClip because of severe, secondary mitral regurgitation, which developed after an initial decline in LV function. In total, 38 patients died during follow-up. One patient died due to acute HF. Other deaths were predominantly related to the underlying oncological disease $(n=30)$ or cancer treatment-related infections $(n=2)$. In 5 patients, the cause of death was unknown.

\section{AICD echocardiographic follow-up and reversibility}

Follow-up echocardiographic examinations were available in 67 patients, with a median follow-up time of I7.7 [IO.5-26.6] months between diagnosis and the last echocardiographic examination. Of the 53 patients who had a normal iEDV at diagnosis, only 2 patients $(3.7 \%)$ developed an iEDV above the upper limit of normal (ULN) during follow-up. Thirteen out of I4 patients who presented with a dilated LV showed normal iEDV values at follow-up. There was no relation between follow-up duration and the change in iEDV ( $\rho=-0.079$; 


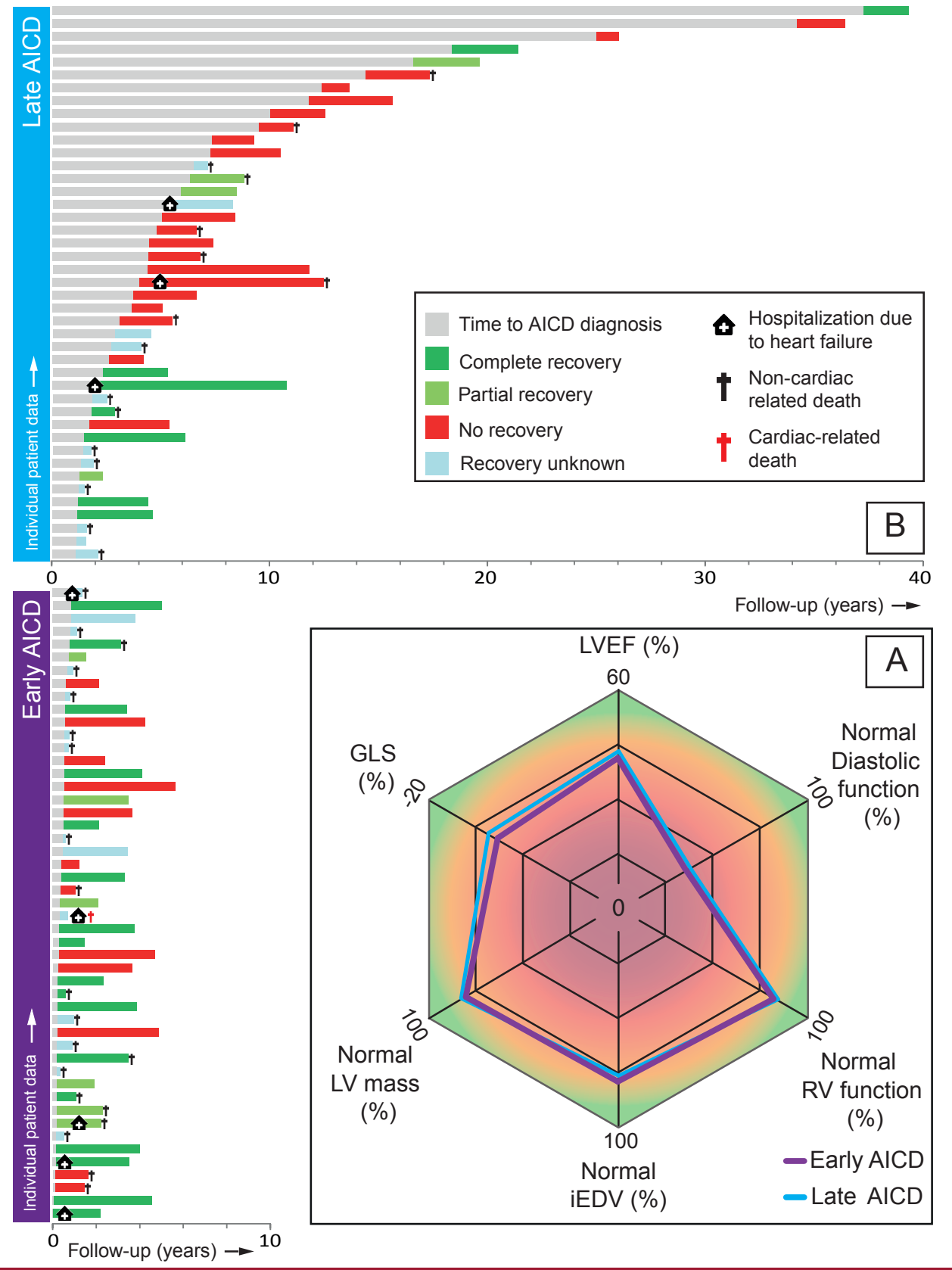

Figure 2 Echocardiographic characterization of early- and late diagnosed anthracycline-induced cardiac dysfunction and follow-up. A) The radar chart shows the echocardiographic phenotype at diagnosis of early- and late AICD, which are both characterized by a mild hypokinetic, non-dilated cardiomyopathy. LVEF and GLS are expressed as group means, LV mass, iEDV and RV function are expressed as \% of patients with normal outcomes and diastolic function is expressed as $\%$ of patients with diastolic dysfunction $\leq$ grade I; B) Individual time periods of time to AICD diagnosis and follow-up outcomes regarding i) hospitalization due to heart failure ii) recovery of LV function and iii) (non-)cardiac death. 
$\mathrm{p}=0.524)$. Sixty-seven patients could be analyzed for reversibility of AICD. Of the 25 patients who were excluded from the analysis, 20 patients died before cardiac follow-up could be performed. In 32 patients (48\%) no recovery of LV function was observed (treatment in $\mathrm{n}=24$ ), Io patients ( $15 \%$ ) had partial recovery (treatment in $\mathrm{n}=9$ ), and 25 patients $(37 \%)$ showed complete recovery (treatment in $n=17$ ). No differences were observed in GLS at diagnosis between patients with- and without partial recovery ((partial) recovery -I3.9 \pm 3.0 ; no recovery -I4.0 $\pm 3.6 ; \mathrm{p}=0.908$ ). Patients with early AICD were more likely to show (partial) recovery of LV function compared to late AICD patients, without significant differences in maximum tolerated doses of HF medication (Table 3).

Table 2 Echocardiographic outcomes of patients with early- and late diagnosed AICD

\begin{tabular}{|c|c|c|c|c|}
\hline Left ventricle & Overall $(n=92)$ & Early $(n=49)$ & Late $(n=43)$ & p-value \\
\hline $\operatorname{IVSd}(\mathrm{mm})$ & $9.7(\mathrm{I} .8)$ & $9.8(\mathrm{I} .7)$ & 9.6 (I.9) & 0.543 \\
\hline PWd (mm) & $9.5(\mathrm{I} .6)$ & 9.6 (I.6) & $9.4(\mathrm{I} .7)$ & 0.483 \\
\hline LVIDd (mm) & $49.7(6.8)$ & $50.0(7 \cdot 3)$ & $49.3(6.3)$ & 0.536 \\
\hline $\begin{array}{l}\text { iLV mass }\left(\mathrm{g} / \mathrm{m}^{2}\right) \\
\text { Increased iLV mass }(\mathrm{n})\end{array}$ & $\begin{array}{c}89.9(23.9) \\
15 / 9 I\end{array}$ & $\begin{array}{c}\text { 9I.3 (2I.I) } \\
8 / \mathrm{I} 7\end{array}$ & $\begin{array}{c}88.4(26.9) \\
\quad 7(\mathrm{I} 7)\end{array}$ & $\begin{array}{l}0.572 \\
0.319\end{array}$ \\
\hline $\begin{array}{l}\mathrm{iEDV}\left(\mathrm{mL} / \mathrm{m}^{2}\right) \\
\text { Dilated }(\mathrm{n})\end{array}$ & $\begin{array}{l}63.3(15.5) \\
19(2 \mathrm{I})\end{array}$ & $\begin{array}{c}63.6 \\
9(\mathrm{I} 8)\end{array}$ & $\begin{array}{l}62.9(16.4) \\
\text { IO }(23)\end{array}$ & $\begin{array}{l}0.840 \\
0.334\end{array}$ \\
\hline $\begin{array}{c}\mathrm{iESV}\left(\mathrm{mL} / \mathrm{m}^{2}\right) \\
\text { Dilated }(\mathrm{n})\end{array}$ & $\begin{array}{l}36.2(\mathrm{II} .2) \\
69(75)\end{array}$ & $\begin{array}{c}35.9(9.6) \\
40(82)\end{array}$ & $\begin{array}{c}36.5(\mathrm{I} 3.0) \\
29(67)\end{array}$ & $\begin{array}{l}0.813 \\
0.178\end{array}$ \\
\hline LVEF (\%) & $43.3(5.5)$ & $43.6(4.9)$ & $43.0(6.2)$ & 0.576 \\
\hline GLS (\%) & $-13.5(3.3)$ & $-\mathrm{I} 3.2(3 . \mathrm{I})$ & $-13.7(3.5)$ & 0.550 \\
\hline \multicolumn{5}{|l|}{ Right ventricle } \\
\hline Basal RV dimension (mm) & $32.8(6.2)$ & $32.6(5.4)$ & $33.0(7.1)$ & 0.756 \\
\hline \multicolumn{5}{|l|}{ RV function $(\mathrm{n}=9 \mathrm{I})$} \\
\hline TAPSE $(\mathrm{mm})$ & $19.5(4.6)$ & I9.5 (4.3) & $19.6(4.9)$ & 0.905 \\
\hline $\mathrm{S}^{\prime}(\mathrm{cm} / \mathrm{sec})$ & I2.I (2.8) & $\mathrm{I} 2.2(2.7)$ & II.9 (2.9) & 0.632 \\
\hline Impaired (n) & $\mathrm{I} 4$ (I5) & 7 (I5) & $7(\mathrm{I} 6)$ & 0.823 \\
\hline Systolic RV pressure (mmHg) & $2 \mathrm{I} .4(5.6)$ & $21.0(5.2)$ & $2 \mathrm{I} .9(6 . \mathrm{I})$ & 0.617 \\
\hline \multicolumn{5}{|l|}{ Diastolic function } \\
\hline LAVI $\left(\mathrm{mL} / \mathrm{m}^{2}\right)$ & $28.6(9 . \mathrm{I})$ & 29.I (8.2) & $28.0(9.9)$ & 0.598 \\
\hline $\mathrm{E}(\mathrm{cm} / \mathrm{sec})$ & $55.0(\mathrm{I} 8.5)$ & $54.7(15.8)$ & $55.5(21.4)$ & 0.840 \\
\hline $\mathrm{A}(\mathrm{cm} / \mathrm{sec})$ & $63.0(17.0)$ & $63.7(17.3)$ & $62.2(\mathrm{I} 6.9)$ & 0.696 \\
\hline E / A ratio & 0.9I (0.39) & $0.9 \mathrm{I}(0.40)$ & 0.9I (0.39) & $0.96 \mathrm{I}$ \\
\hline E deceleration time (msec) & $176(5 \mathrm{I})$ & I82 (6o) & $169(37)$ & $0.27 \mathrm{I}$ \\
\hline $\mathrm{E}^{\prime}(\mathrm{cm} / \mathrm{sec})$ & 8.I (2.9) & $8.0(3.0)$ & $8.2(2.8)$ & 0.747 \\
\hline $\mathrm{E} / \mathrm{E}^{\prime}$ & $7.2(2.6)$ & $7.3(2.5)$ & 7.I (2.7) & $0.77 \mathrm{I}$ \\
\hline \multicolumn{5}{|c|}{$\begin{array}{l}\text { Categorical variables are expressed as n (\%) and continuous variables as mean (standard deviation) or median [interquartile } \\
\text { range]. IVSd = end-diastolic intraventricular septal dimension; PWd = end-diastolic posterior wall dimension; LVEDD = } \\
\text { left ventricular end-diastolic dimension; iLV: indexed left ventricular (mass); iEDV = indexed end-diastolic volume; iESV = } \\
\text { indexed end-systolic volume; LVEF = left ventricular ejection fraction; GLS = global longitudinal strain; RV = right ventricle; } \\
\text { TAPSE = tricuspid annular plain systolic excursion; S' = Doppler Tissue Imaging-derived S-wave; LAVI = left atrial volume } \\
\text { index; }\end{array}$} \\
\hline
\end{tabular}



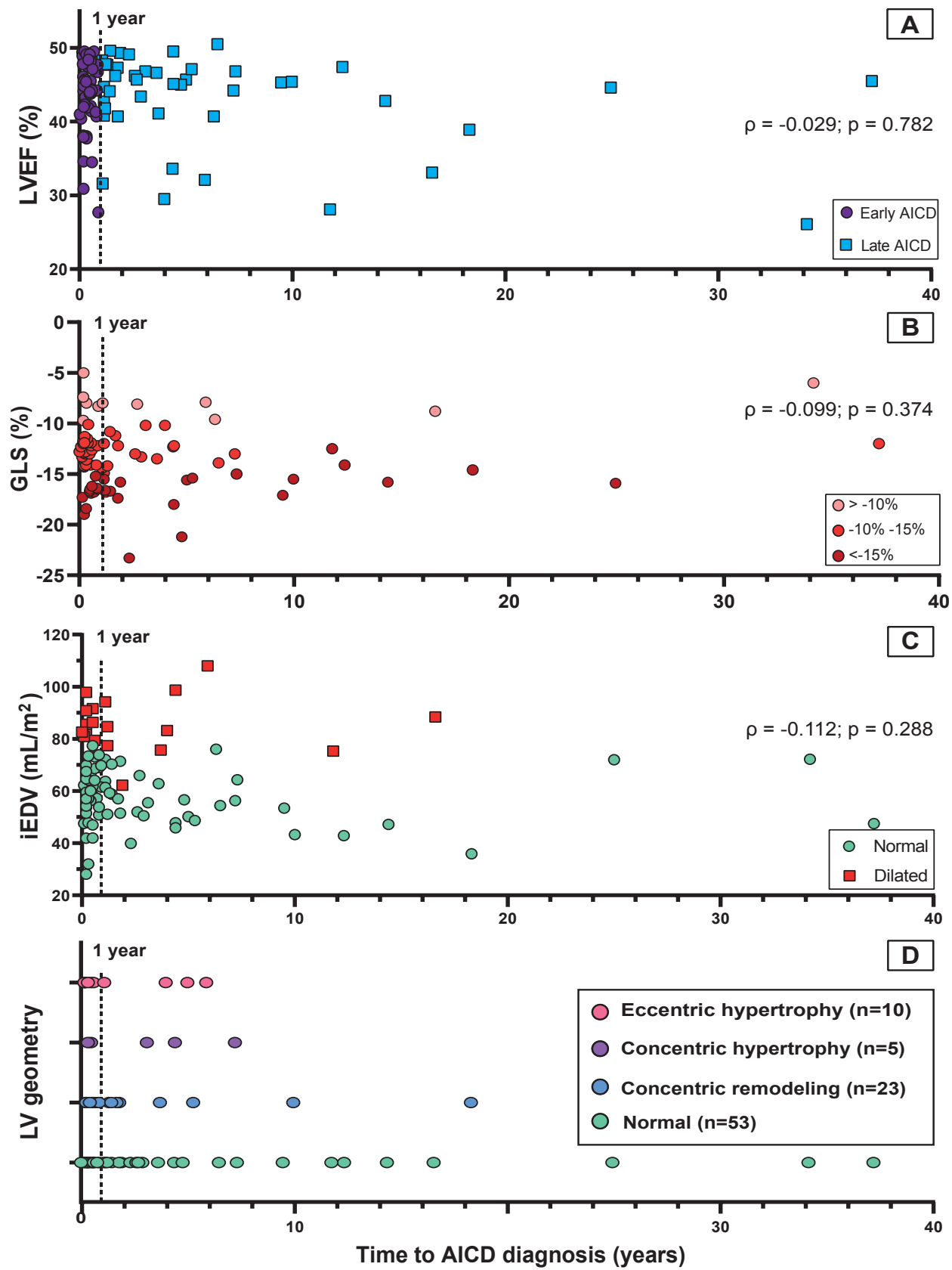

Figure 3 Echocardiographic outcomes at time of diagnosis. The dashed line represents the time-point of I year, which is used to differentiate between early- and late AICD. A) Left ventricular ejection fraction (LVEF); B) Global longitudinal strain (GLS); C) End-diastolic volume, indexed for body surface area (iEDV) and classified as 'normal' or 'dilated'; D) LV geometry, based on LV dimension and LV mass. 
Table 3 Heart failure therapy and response to heart failure treatment

\begin{tabular}{|c|c|c|c|c|}
\hline Heart failure therapy & Overall $(n=92)$ & Early $(n=49)$ & Late $(n=43)$ & p-value \\
\hline \multicolumn{5}{|l|}{ Heart failure treatment initiation } \\
\hline Initiated & $63(68)$ & $36(73)$ & $27(63)$ & \multirow{3}{*}{0.258} \\
\hline Adding to previous therapy & $5(5)$ & I (2) & $4(9)$ & \\
\hline LVEF $>45 \%$ and no cardiac complaints & $24(26)$ & I2 (24) & I2 (28) & \\
\hline \multicolumn{5}{|l|}{ Number of HF drugs } \\
\hline I & $32(47)$ & $20(54)$ & I2 (39) & \multirow{3}{*}{0.162} \\
\hline 2 & $28(4 \mathrm{I})$ & $15(4 \mathrm{I})$ & I2 (42) & \\
\hline 3 & $8(\mathrm{I} 2)$ & $2(5)$ & $6(19)$ & \\
\hline \multicolumn{5}{|l|}{ ACE inhibitors / ARB } \\
\hline $\mathrm{n}$ & 6I (90) & $34(92)$ & $27(87)$ & \multirow[t]{2}{*}{0.538} \\
\hline$\%$ of target dose & $25[\mathrm{I} 6.7-50]$ & $25[\mathrm{I} 6.7-50]$ & $25[25-4 \mathrm{I} \cdot 5]$ & \\
\hline \multicolumn{5}{|l|}{ Beta-blockers } \\
\hline $\mathrm{n}$ & $39(57)$ & I8 (49) & $27(87)$ & \multirow[t]{2}{*}{0.183} \\
\hline$\%$ of target dose & $25[12.5-50]$ & I8.75 [12.5-25] & $25[12.5-50]$ & \\
\hline \multicolumn{5}{|l|}{ MRA } \\
\hline & $6(9)$ & $2(5)$ & $4(\mathrm{I} 3)$ & \\
\hline$\%$ of target dose & $37.5[25-50]$ & $37.5[25-50]$ & $37.5[25-50]$ & \\
\hline \multicolumn{5}{|l|}{ Side effects } \\
\hline Hypotension & I8 (26) & IO $(27)$ & $8(26)$ & \\
\hline Renal dysfunciton & $3(4)$ & I (3) & $2(6)$ & \\
\hline Other & $6(9)$ & $3(8)$ & $3(\mathrm{IO})$ & \\
\hline Duration of HF treatment (months) & I6.8 [4.8-29.8] & I4.6 $[3.4-28.0]$ & $25.3[$ IO.I-35.2] & $0.038^{*}$ \\
\hline Response to HF treatment & Overall $(n=49)$ & $\begin{array}{l}\text { (partial) reco- } \\
\text { very }(n=25)\end{array}$ & $\begin{array}{c}\text { No recovery } \\
(n=24)\end{array}$ & p-value \\
\hline Early & $25(5 \mathrm{I})$ & I7 (68) & $8(33)$ & $0.015^{*}$ \\
\hline Late & $24(49)$ & $8(32)$ & I6 (67) & \\
\hline \multicolumn{5}{|l|}{ Number of HF drugs } \\
\hline I & 2I (43) & II (44) & IO $(42)$ & \multirow{3}{*}{0.728} \\
\hline 2 & $20(4 \mathrm{I})$ & $9(36)$ & II (46) & \\
\hline 3 & $8(\mathrm{I} 6)$ & $5(20)$ & $3(12)$ & \\
\hline \multicolumn{5}{|l|}{ ACE inhibitors / ARB } \\
\hline & $46(94)$ & $23(92)$ & $23(96)$ & \multirow[t]{2}{*}{0.316} \\
\hline$\%$ of target dose & $25[\mathrm{I} 6.7-50]$ & $25[12.5-50]$ & $25[25-50]$ & \\
\hline \multicolumn{5}{|l|}{ Beta-blockers } \\
\hline $\mathrm{n}$ & $29(59)$ & $\mathrm{I} 5(60)$ & I4 (58) & \multirow[t]{2}{*}{0.713} \\
\hline$\%$ of target dose & $25[\mathrm{I} 2.5-50]$ & $25[12.5-50]$ & $37.5[12.5-50]$ & \\
\hline Duration of HF treatment (months) & $24.6[\mathrm{I} 3.5-35.2]$ & $22.3[13.5-30.9]$ & $25.9[13.3-36.6]$ & $0.6 \mathrm{I} 7$ \\
\hline \multicolumn{5}{|c|}{$\begin{array}{l}\text { Target doses are expressed as percentage of target dose according to the ESC Guideline on Heart Failure.Io Categorical va- } \\
\text { riables are expressed as } \mathrm{n}(\%) \text { and continuous variables as mean (standard deviation) or median [interquartile range]. LVEF } \\
=\text { left ventricular ejection fraction; } \mathrm{HF}=\text { heart failure; ACE = angiotensin converting enzyme; ARB = angiotensin II receptor } \\
\text { blockers; MRA = mineralocorticoid receptor antagonist; " p-value }<0.05 .\end{array}$} \\
\hline
\end{tabular}




\section{Discussion}

The aim of this consecutive cohort study was to evaluate the echocardiographic phenotype of AICD, establish what impact a delayed diagnosis had on the degree of LV dysfunction, LV dimensions and response to HF treatment. The key findings of this study are threefold: (I) the majority of patients presented with mild LV dysfunction without LV dilatation (2) the echocardiographic phenotype was not different in patients diagnosed with early or late AICD and (3) patients with an early AICD diagnosis and prompt initiation of HF treatment were more likely to have a (partial) recovery of LV function, compared to patients with a late AICD diagnosis.

In contrast to previous studies in which AICD is described as a toxic cause of DCM [I5], we found that LV dilatation was present in only one-fifth of cases at diagnosis and only two patients developed LV dilatation during a median follow-up of 17.7 months. We believe that there are three major reasons for the absence of a DCM-like phenotype. Firstly, many patients received an early diagnosis of AICD due to serial echocardiographic screening at a cardio-oncology clinic. In the past, the diagnosis of AICD was often established upon the development of symptomatic HF, and subclinical changes in LV function were not detected. In these patients, prolonged activation of compensatory mechanisms, including the renin-angiotensin aldosterone system (RAAS), and subsequent LV remodeling, may have led to a more pronounced dilatation of the LV. However, in our study, dilatation was also not present in the majority of patients with a late AICD diagnosis. The second possible reason for the absence of abnormal dimensions could be related to the reduction of cumulative anthracycline dose over the last decades. While doxorubicin doses exceeding $>500 \mathrm{mg} / \mathrm{m}^{2}$ were commonly administered in the past [I], the maximum cumulative dose of this agent is nowadays restricted to $450 \mathrm{mg} / \mathrm{m}^{2}$ [16], with a median dose of 329 [200-329] $\mathrm{mg} / \mathrm{m}^{2}$ in this study ( $\mathrm{n}=9$ receiving $\geq 450 \mathrm{mg} / \mathrm{m}^{2}$ ). It is plausible that these dose restrictions have resulted in an overall milder AICD phenotype. Thirdly, in patients developing LV dysfunction with an LVEF $<45 \%$, HF treatment was promptly initiated, aiming at suppression of RAAS to prevent adverse LV remodeling. The early detection of AICD has shown to be beneficial in one study, where patients with early initiation of therapy were more likely to respond to pharmacological treatment [I7]. To our knowledge, our study is the first to validate this time-dependent response to HF treatment. In conclusion, an earlier diagnosis, a restriction in the maximum cumulative anthracycline dose and the initiation of HF treatment might jointly have led to a hypokinetic non-dilated cardiomyopathy rather than DCM [I8].

We did not find any echocardiographic differences in dimension and function between patients diagnosed early vs. late (Table 2). Furthermore, there were also no discrepancies in LV mass between the two groups. To date, a number of imaging studies have evaluated this parameter in AICD both by CMR [I9-2I] and echocardiography [22-23]. Currently, CMR is considered as the gold standard for measurements of cardiac structure and volumes [I3]. With this technique, three studies found a decrease in LV mass [I9-2I], and an inverse correlation with the anthracycline dose [20]. Furthermore, a lower LV mass was predictive of cardiovascular death, appropriate ICD therapy and HF hospitalization in a multivariate model [20]. However, these findings are contradictory to echocardiographic 
studies which found an increase in LV mass [22-23]. Armstrong et al. studied adults who were treated with anthracyclines during childhood. They found a reduced LV mass in nearly half of patients [24]. Comparison of CMR with echocardiography performed within 48 hours, revealed that echocardiography overestimated LVEF and LV mass and underestimates LV volumes. The absence of reduced LV masses in our study population could therefore be related to LV mass measurements with echocardiography.

In contrast to our hypothesis, our data does not support the progressive nature of AICD regarding cardiac remodeling. To date, longitudinal imaging studies in patients with AICD are scarce, with a small number of studies performed in pediatric- [25] and adult cancer patients [22,26]. Lipshultz et al. prospectively followed II5 survivors of childhood acute leukemia with serial echocardiograms during a median follow-up of II.8 years [25]. The LV contractility initially declined after doxorubicin containing chemotherapy, normalized the next 6 years and subsequently became significantly depressed >I 2 years after the cancer diagnosis. In another prospective cohort study among 277 breast cancer patients treated with doxorubicin ( $36 \%$ in combination with trastuzumab) the LVEF decline was not progressive during a median follow-up of 2 years [22]. In a study by Jones et al. in I43 patients that were followed for 2 years, none transitioned to more advanced HF stages [26]. In our study only $5.5 \%$ of patients progressed to symptomatic HF. Nevertheless, the presence of asymptomatic LV dysfunction gives an increased risk of ultimately progressing to symptomatic HF. In a meta-analysis evaluating the risk in patients with systolic LV dysfunction due to various etiologies, the incidence of symptomatic HF was 8.4 (95\% CI 4.0-I2.8) per IOO person-years, compared to I.O4 (95\% CI 0.0-2.2) per IOo person-years in the absence of LV dysfunction, equaling a relative risk of 4.6 (95\% CI 2.2-9.8) [27]. This meta-analysis illustrates the importance of implementing effective strategies in the pre-symptomatic stages to mitigate the progression rate to symptomatic HF. This progression might also be dependent on the development of other cardiac stressors, such as hypertension, valvular disease, CAD, or the presence of pathogenic variants in cardiomyopathy-associated genes [28-29]. In the absence of these so called "second-hits", it is possible that a considerable proportion of patients only develops mild LV dysfunction after anthracycline-containing chemotherapy, that remains stable for years. Larger observational cohort studies, preferably with long-term follow-up can shed more light on the natural course of this specific disease entity.

\section{Limitations}

Our analysis was restricted to patients that visited the cardio-oncology outpatient clinic. Patients treated with potentially cardiotoxic cancer therapy prior to its launch currently receive cardiological follow-up to screen for long-term cardiovascular complications. It is possible that patients who developed a more severe AICD phenotype presented at the emergency care unit earlier and were never seen in an outpatient clinic setting and therefore were not included in our study. Furthermore, the current study used patient information collected during standard clinical care. Albeit the follow-up of these patients is standardized to a great extent [Io], there still was heterogeneity in the data. Even though 
current guidelines recommend to perform cardiac screening prior to treatment [30], many patients were referred after initiation of anthracycline-treatment, and therefore lacked cardiac baseline assessment. Subtle changes in LV dimensions and -volumes within patients could therefore have been overlooked. Also, additional testing for other causes of impaired LV function, such as CAD, had not (yet) been performed in a number of patients, leaving uncertainty of the diagnosis of AICD. Nevertheless, this represents only a small subset of our cohort where, from a clinical point of view, AICD was very likely.

Biomarkers, such as troponin or BNP, are not routinely performed at our cardio-oncology clinic and were only available in a limited number of patients. We therefore were unable to include the outcomes of biomarkers in our analysis.

\section{Conclusions}

We found that the AICD phenotype overall was mild and a majority of patients lacked cardiac complaints. In the absence of serial echocardiographic assessment, it therefore is plausible that the impaired cardiac function would have remained undetected. Since response to HF treatment is time-dependent, detection of asymptomatic LV dysfunction is of great importance. Furthermore, when cardiac compromise is detected, other cardiovascular risk factors can be treated more aggressively, potentially decreasing the risk of patients progressing to more advanced HF stages. Moreover, if a patient is planned to receive further cardiotoxic treatment, preventive actions can be considered [30]. The involvement of cardiologist during potentially cardiotoxic treatment processes seems valuable to provide adequate cardiac screening, as well as to give optimal heart failure treatment for those patients who eventually develop cardiac dysfunction [3I].

Importantly, future research within the field of cardio-oncology should not only focus on the added value of cardiac screening, but also possible drawbacks including medicalization and increased health-care costs [32]. Trials evaluating different follow-up strategies are required to achieve an optimal risk-benefit ratio. It is plausible that the optimal strategy varies per cancer type, as often the prognosis of the malignancy is a dominant factor. Longitudinal cohort studies establishing more insight into the natural course of AICD are herein of pivotal importance. 


\section{References}

I Von Hoff DD, Layard MW, Basa P, et al. Risk factors for doxorubicin-induced congestive heart failure. Ann Intern Med.I979;9I:710-717.

2 Lotrionte M, Biondi-Zoccai G, Abbate A, et al. Review and meta-analysis of incidence and clinical predictors of anthracycline cardiotoxicity. Am J Cardiol. 2013;II2:1980-4.

3 Cardinale D, Colombo A, Bacchiani G, et al. Early Detection of Anthracycline Cardiotoxicity and Improvement With Heart Failure Therapy. Circulation. 2015;I3I(22):198I-8.

4 Fornaro A, Olivotto I, Rigacci L, et al. Comparison of long-term outcome in anthracycline-related versus idiopathic dilated cardiomyopathy: a single centre experience. Eur J Heart Fail. 2018;20(5):898906.

5 Felker GM, Thompson RE, Hare JM, et al. Underlying causes and long-term survival in patients with initially unexplained cardiomyopathy. N Engl J Med. 2000;342(15):1077-84.

6 Cardinale D, Sandri MT, Colombo A, et al. Prognostic value of troponin I in cardiac risk stratification of cancer patients undergoing high-dose chemotherapy. Circulation. 2004;I09(22):2749-54.

7 Auner HW, Tinchon C, Linkesch W, et al. Prolonged monitoring of troponin T for the detection of anthracycline cardiotoxicity in adults with hematological malignancies. Ann Hematol. 2003;82(4):21822.

8 Mann DL, Bristow MR. Mechanisms and Models in Heart Failure. Circulation. 2005;1II:2837-2849.

9 Mercurio V, Pirozzi F, Lazzarini E, et al. Models of heart failure based on the cardiotoxicity of anticancer drugs. J Card Fail. 2016;22(6):449-58. doi: Io.IoI6/j.cardfail.20I6.04.008

Io Teske AJ, Linschoten M, Kamphuis JAM, et al. Cardio-oncology: an overview on outpatient management and future developments. Neth Heart J. 2018;26(II):52I-532.

II Ponikowski P, Voors AA, Anker SD, et al. 20I6 ESC Guidelines for the diagnosis and treatment of acute and chronic heart failure: The Task Force for the diagnosis and treatment of acute and chronic heart failure of the European Society of Cardiology (ESC). Developed with the special contribution of the Heart Failure Association (HFA) of the ESC. Eur J Heart Fail. 20I6;I8(8):89I-975. doi: Io.Ioo2/ejhf.592

I2 Zamorano JL, Lancellotti P, Rodriguez Muñoz D, et al. 20I6 ESC Position Paper on cancer treatments and cardiovascular toxicity developed under the auspices of the ESC Committee for Practice Guidelines: The Task Force for cancer treatments and cardiovascular toxicity of the European Society of Cardiology (ESC). Eur Heart J. 2016;37(36):2768-28oI.

I3 Plana JC, Galderisi M, Barac A, et al. Expert Consensus for Multimodality Imaging Evaluation of Adult Patients during and after Cancer Therapy: A Report from the American Society of Echocardiography and the European Association of Cardiovascular Imaging. Eur Heart J Cardiovasc Imaging. 2014;15(I0):I063-93.

I4 Shankar SM, Marina N, Hudson MM, et al. Monitoring for Cardiovascular Disease in Survivors of Childhood Cancer: Report From the Cardiovascular Disease Task Force of the Children's Oncology Group. Pediatrics. 2008;I2I(2):e387-96.

I5 Japp AG, Gulati A, Cook SA, Cowie MR, Prasad SK. The Diagnosis and Evaluation of Dilated Cardiomyopathy. J Am Coll Cardiol. 20I6;67(25):2996-30I0.

I6 Ewer MS, Ewer SM. Cardiotoxicity of anticancer treatments. Nat Rev Cardiol. 2015;I2(9):547-58.

I7 Cardinale D, Colombo A, Lamantia G, et al. Anthracycline-induced cardiomyopathy: clinical relevance and response to pharmacologic therapy. J Am Coll Cardiol.20I0;55(3):213-20.

I8 Pinto YM, Elliott PM, Arbustini E, et al. Proposal for a revised definition of dilated cardiomyopathy, hypokinetic non-dilated cardiomyopathy, and its implications for clinical practice: a position statement of the ESC working group on myocardial and pericardial diseases. Eur Heart J. 2016;37(23):1850-8.

I9 Ferreira de Souza T, Quinaglia AC, Silva T, et al. Anthracycline Therapy Is Associated With Cardiomyocyte Atrophy and Preclinical Manifestations of Heart Disease. JACC Cardiovasc Imaging. 20I8;II(8):I045-55.

20 Neilan TG, Coelho-Filho OR, Pena-Herrera D, et al. Left Ventricular Mass in Patients With a Cardiomyopathy After Treatment With Anthracyclines. Am J Cardiol. 20I2;IIO(II):I679-86.

2I Jordan JH, Castellino SM, Meléndez GC, et al. Left Ventricular Mass Change After Anthracycline Chemotherapy. Circ Heart Fail. 20I8;II(7):eoo456o. 
22 Narayan HK, Finkelman B, French B, Pet al. Detailed Echocardiographic Phenotyping in Breast Cancer Patients: Associations With Ejection Fraction Decline, Recovery, and Heart Failure Symptoms Over 3 Years of Follow-Up. Circulation. 20I7;I35(I5):I397-I4I2.

23 Tan TC, Bouras S, Sawaya H, et al. Time Trends of Left Ventricular Ejection Fraction and Myocardial Deformation Indices in a Cohort of Women with Breast Cancer Treated with Anthracyclines, Taxanes, and Trastuzumab. J Am Soc Echocardiogr. 2015;28(5):509-I4.

24 Armstrong GT, Plana JC, Zhang N, et al. Screening Adult Survivors of Childhood Cancer for Cardiomyopathy: Comparison of Echocardiography and Cardiac Magnetic Resonance Imaging. J Clin Oncol. 2012;30(23):2876-84

25 Lipshultz SE, Lipsitz SR, Sallan SE, et al. Chronic Progressive Cardiac Dysfunction Years After Doxorubicin Therapy for Childhood Acute Lymphoblastic Leukemia. J Clin Oncol. 2005;23(I2):262936.

26 Jones DN, Jordan JH, Meléndez GC, et al. Frequency of Transition From Stage A to Stage B Heart Failure After Initiating Potentially Cardiotoxic Chemotherapy. JACC Heart Fail. 20I8;6(I2):IO23-IO32.

27 Echouffo-Tcheugui JB, Ergou S, Butler J, Yancy CW, Fonarow GC. Assessing the Risk of Progression From Asymptomatic Left Ventricular Dysfunction to Overt Heart Failure: A Systematic Overview and Meta-Analysis. JACC Heart Fail. 20I6;4(4):237-48.

28 Linschoten M, Teske AJ, Cramer MJ, van der Wall E, Asselbergs FW. Chemotherapy-Related Cardiac Dysfunction: A Systematic Review of Genetic Variants Modulating Individual Risk. Circ Genom Precis Med. 20I8;II(I):eool753.

29 Garcia-Pavia P, Kim Y, Restrepo-Cordoba MA, et al. Genetic Variants Associated With Cancer Therapy-Induced Cardiomyopathy. Circulation. 20I9;I4O(I):3I-4I.

30 Curigliano G, Cardinale D, Suter T, et al. Cardiovascular toxicity induced by chemotherapy, targeted agents and radiotherapy: ESMO Clinical Practice Guidelines. Ann Oncol. 2012;23 suppl 7:viiis566.

3I Ammon M, Arenja N, Leibundgut G, Buechel RR, Kuster GM, Kaufmann BA, et al. Cardiovascular management of cancer patients with chemotherapy-associated left ventricular systolic dysfunction in real-world clinical practice. J Card Fail. 2013;19(9):629-34. doi: Io.IoI6/j.cardfail.2013.07.007

32 Levis BE, Binkley PF, Shapiro CL. Cardiotoxic effects of anthracycline-based therapy: what is the evidence and what are the potential harms? Lancet Oncol. 20I7;18(8):e445-e456. doi: Io.IoI6/SI4702045(I7)30535-I 
Supplemental Table I Reference values of echocardiographic measurements and international guidelines on echocardiographic examination.

\begin{tabular}{|c|c|c|}
\hline & Lower Limit of Normal & Upper Limit of Normal \\
\hline \multicolumn{3}{|l|}{ Left ventricle } \\
\hline $\begin{array}{l}\operatorname{iEDV}(2 \mathrm{D}) \\
\operatorname{iEDV}(3 \mathrm{D})\end{array}$ & $\begin{array}{ll}\text { Male: } & 34 \mathrm{~mL} / \mathrm{m}^{2} \\
\text { Female: } & 29 \mathrm{~mL} / \mathrm{m}^{2}\end{array}$ & $\begin{array}{ll}\text { Male: } & 74 \mathrm{~mL} / \mathrm{m}^{2} \\
\text { Female: } & 6 \mathrm{I} \mathrm{mL} / \mathrm{m}^{2} \\
\text { Male: } & 79 \mathrm{~mL} / \mathrm{m}^{2} \\
\text { Female: } & 7 \mathrm{I} \mathrm{mL} / \mathrm{m}^{2}\end{array}$ \\
\hline $\begin{array}{l}\mathrm{iESV}(2 \mathrm{D}) \\
\mathrm{iESV}\left({ }_{3} \mathrm{D}\right)\end{array}$ & $\begin{array}{l}\text { Male: } \quad \text { II } \mathrm{mL} / \mathrm{m}^{2} \\
\text { Female: }\end{array}$ & $\begin{array}{ll}\text { Male: } & 3 \mathrm{I} \mathrm{mL} / \mathrm{m}^{2} \\
\text { Female: } & 24 \mathrm{~mL} / \mathrm{m}^{2} \\
\text { Male: } & 32 \mathrm{~mL} / \mathrm{m}^{2} \\
\text { Female: } & 28 \mathrm{~mL} / \mathrm{m}^{2}\end{array}$ \\
\hline iLV mass* & $\begin{array}{ll}\text { Male: } & 49 \mathrm{~g} / \mathrm{m}^{2} \\
\text { Female: } & 43 \mathrm{~g} / \mathrm{m}^{2}\end{array}$ & $\begin{array}{ll}\text { Male: } & \text { II } 5 \mathrm{~g} / \mathrm{m}^{2} \\
\text { Female: } & 95 \mathrm{~g} / \mathrm{m}^{2}\end{array}$ \\
\hline $\begin{array}{l}\text { Left ventricular ejection fraction } \\
\text { Normal } \\
\text { Mildly impaired } \\
\text { Moderately impaired } \\
\text { Severely impaired }\end{array}$ & $\begin{array}{l}\geq 53 \% \\
40-52 \% \\
30-40 \% \\
<30 \%\end{array}$ & \\
\hline $\begin{array}{l}\text { LV geometry } \\
\text { Normal } \\
\text { Concentric remodeling } \\
\text { Concentric hypertrophy } \\
\text { Eccentric hypertrophy }\end{array}$ & \multicolumn{2}{|c|}{$\begin{array}{l}\text { Normal LV mass and RWT }<0.42 \\
\text { Normal LV mass and RWT }>0.42 \\
\text { Increased LV mass and RWT }>0.42 \\
\text { Increased LV mass and RWT }<0.42\end{array}$} \\
\hline \multicolumn{3}{|l|}{ Right ventricular function } \\
\hline TAPSE & \multicolumn{2}{|l|}{$17 \mathrm{~mm}$} \\
\hline $\mathrm{S}^{\prime}$ & \multicolumn{2}{|l|}{$9.5 \mathrm{~cm} / \mathrm{sec}$} \\
\hline \multicolumn{3}{|c|}{$\begin{array}{l}\text { International guidelines used for the echocardiographic analysis } \\
\text { - Lang RM, Badano LP, Mor-Avi V, Afilalo J, Armstrong A, Ernande L, Flachskampf FA, Foster E, Goldstein SA, Kuznetsova T, } \\
\text { Lancellotti P, Muraru D, Picard MH, Rietzschel ER, Rudski L, Spencer KT, Tsang W, Voigt JU. Recommendations for cardiac } \\
\text { chamber quantification by echocardiography in adults: an update from the American Society of Echocardiography and the } \\
\text { European Association of Cardiovascular Imaging. Eur Heart J Cardiovasc Imaging. 20I5;I6(3):233-70 } \\
\text { - Nagueh SF, Smiseth OA, Appleton CP, Byrd BF, Dokainish H, Edvardsen T, Flachskampf FA, Gillebert TC, Klein AL, Lan- } \\
\text { cellotti P, Marino P, Oh JK, Popescu BA, Waggoner AD. Recommendations for the Evaluation of Left Ventricular Diastolic } \\
\text { Function by Echocardiography: An Update from the American Society of Echocardiography and the European Association } \\
\text { of Cardiovascular Imaging. Eur Heart J Cardiovasc Imaging. 20I6;I7(I2):I32I-I36o. } \\
\text { - Lancellotti P, Tribouilloy C, Hagendorff A, Moura L, Popescu BA, Agricola E, Monin JL, Pierard LA, Badano L, Zamorano } \\
\text { JL. European Association of Echocardiography recommendations for the assessment of valvular regurgitation. Part I: aortic } \\
\text { and pulmonary regurgitation (native valve disease). Eur J Echocardiogr. 2OIO; II(3):223-44 } \\
\text { - Lancellotti P, Moura L, Pierard LA, Agricola E, Popescu BA, Tribouilloy C, Hagendorff A, Monin JL, Badano L, Zamorano } \\
\text { JL. European Association of Echocardiography recommendations for the assessment of valvular regurgitation. Part 2: mitral } \\
\text { and tricuspid regurgitation (native valve disease). Eur J Echocardiogr. 2OIO;II(4):307-32 } \\
\text { - Baumgartner H, Hung J, Bermejo J, Chambers JB, Edvardsen T, Goldstein S, Lancellotti P, LeFevre M, Miller F, Otto CM. } \\
\text { Echocardiographic assessment of valve stenosis: EAE/ASE recommendations for clinical practice. Eur J Echocardiogr. } \\
\text { 2009;IO(I):I-25 }\end{array}$} \\
\hline $\begin{array}{l}\text { "Based on the Cube formula (Lang } \\
\text { iLV = indexed left ventricular (mass) } \\
\text { systolic excursion; } S \text { ' = Doppler Tiss }\end{array}$ & $\begin{array}{l}\mathrm{DV}=\text { indexed end-diastol } \\
\text { ntricle; RWT = relative wa } \\
\text { derived S-wave; }\end{array}$ & $\begin{array}{l}\text { SV = indexed end-systolic volume; } \\
\text { TAPSE = tricuspid annular plain }\end{array}$ \\
\hline
\end{tabular}




\section{Chapter 6}

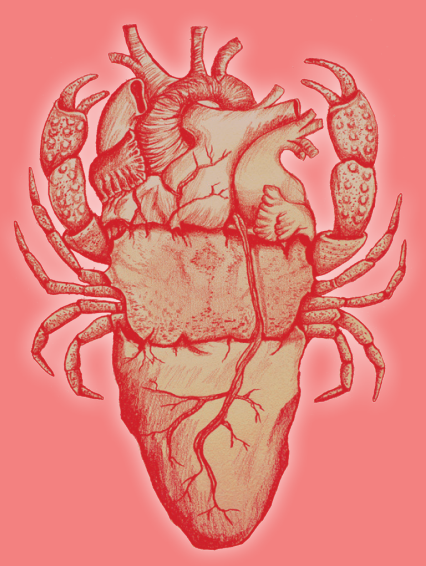




\title{
Cardio-oncology in a real-world setting The clinical yield of specialized cardiac care for cancer patient
}

\author{
Submitted
}

JAM Kamphuis' ${ }^{1}$, M Linschoten ${ }^{1}$, MJ Cramer ${ }^{1}$, PA Doevendans ${ }^{1,2,3}$, FW Asselbergs ${ }^{1,4,5}$, AJ Teske ${ }^{1}$

I Department of Cardiology, Division of Heart and Lungs, University Medical Centre Utrecht, Utrecht, The Netherlands 2 Netherlands Heart Institute, Utrecht, The Netherlands

3 Central Military Hospital, Utrecht, The Netherlands

4 Health Data Research UK and Institute of Health Informatics, University College London, London, UK

5 Institute of Cardiovascular Science, Faculty of Population Health Sciences, University College London, London, UK 


\begin{abstract}
Background Cardio-oncology clinics strive to optimize cardiovascular health of cancer patient prior-, during- and after cancer treatment. Up to now, limited studies are available that present data of clinical cardio-oncology experiences.
\end{abstract}

Objectives This observational study presents experiences of providing real-world cardio-oncological care. We describe our population, the incidence of cardiovascular toxicities and its influence on oncological treatment.

Methods All patients referred to the cardio-oncology outpatient service at the University Medical Center Utrecht, the Netherlands, from April 2015 to September 2019 were included. The main focus of this service lies on early detection of cancer therapy-related cardiac dysfunction (CTRCD), as well as screening for long-term cardiovascular complications. Outcomes measures included, amongst others, the occurrence of CTRCD and other cardiovascular adverse events (CVAE), and the impact on oncological treatment.

Results 800 patients visited the cardio-oncology service, with a median follow-up time of I4.3 [IQR 5.5-28.0] months. Hematological malignancies (65\%) and breast cancer (24\%) were the most prevalent malignancies. $182(23 \%)$ experienced a CVAE during oncological treatment and IO4 (I3\%) after treatment. In I45 patients (80\%), the CVAE did not influence oncological treatment, whereas treatment was interrupted in $7(4 \%)$ patients and discontinued in 30 (I6\%) patients. I76 patients experienced CTRCD, of which $96(55 \%)$ and 60 (34\%) had no- or mild cardiac complaints, respectively.

Conclusion CVAEs were commonly detected at our specialized cardio-oncology service, and $84 \%$ of patients were able to complete cancer treatment. Whether specialized cardio-oncological care has beneficial effects on both long-term cardiac- and oncological outcome, warrants further investigation. 


\section{Introduction}

Over the past decades, improvements in cancer screening methods and development of new treatment strategies has led to rapidly increasing numbers of cancer survivors worldwide [I,2]. In addition, in several patients with incurable forms of cancer, the disease can be controlled and managed over considerable periods of time. In the light of these developments, the prevention and management of short- and long-term treatment-related toxicity is gaining importance. Cardiovascular toxicity is a feared side effect of cancer therapy and can be associated with considerable morbidity and mortality during and after cancer treatment $[3,4]$. Depending on the type of antineoplastic treatment, cardiovascular toxicity can manifest in various different forms, including arrhythmia's, pericardial disease, cancer therapy-related cardiac dysfunction (CTRCD) and heart failure, coronary artery disease, and hypertension.

Cardio-oncology is a fast-growing subspecialty that focuses on the prevention, diagnosis and management of these side effects. The Task Force for cancer treatment and cardiovascular toxicity of the European Society of Cardiology published a position paper in 2016 in which the different steps of cardiovascular monitoring and decision-making regarding cancer treatment are reviewed [3]. However, as this field is still in its infancy with few clinical trials yet conducted, the recommendations in this position paper are based on expert opinion. Notwithstanding, the numerous specialized cardio-oncology services established around the globe handle different referral policies, screening methods, and treatment strategies, according to local need and -preference. Thus far, the number of studies that present data of clinical cardio-oncology experiences are still scarce $[5,6]$.

Here we show our first results and experiences regarding our cardio-oncological service. With this observational study, we aim to i) describe our patient population and referral reasons and ii) present the incidence of various forms of cardiovascular toxicity and their effect on oncological and cardiovascular treatment.

\section{Methods}

This observational study included all patients consecutively referred to the cardio-oncology outpatient service at the University Medical Center Utrecht and the Bergman Clinics, Bilthoven, the Netherlands, from April 2015 to September 2019. The protocol of this clinic has been described in detail previously [7]. The cardio-oncology service handles a one-stop-shop principle including a I2-lead resting electrocardiogram (ECG) and echocardiogram including a $3 \mathrm{D}$ left ventricular ejection fraction (LVEF) measurement and global longitudinal strain (GLS) analysis, followed by a visit to the cardiologist. Per week, approximately 3-4 new patients are referred. The main focus of this service lies on the early detection of CTRCD through serial echocardiographic assessments. A risk stratification model, which incorporates patient- and treatment-related risk factors (Chapter 2 Table 2) is used for CTRCD risk stratification [8]. The risk stratification model is used by haematologists and oncologist to determine the a priori risk of CTRCD and the need for referral to the 
cardio-oncology service. Additionally, this risk stratification model serves as a guidance to determine the frequency of visits during follow-up. In addition to the early detection of CTRCD, cancer patients and survivors are also referred for screening purposes for the detection of long-term cardiovascular complications.

\section{Patient population}

The largest proportion of patients seen at our cardio-oncology service belong to one of the following groups:

\section{i) Trastuzumab}

According to the Food and Drug Administration [9] and oncology guidelines [Io], quarterly assessment of cardiac function is recommended in patients receiving trastuzumab. Historically, multigated acquisition scans (MUGA) scans were frequently used for this purpose, but in recent years this has slowly shifted towards the use of non-invasive, radiation-avoiding, 3-dimensional echocardiography. At the launch of the cardio-oncology service in 2015, the LVEF was still monitored with MUGA scans and patients were predominantly first referred upon the development of a significant decline. In recent years, this approach has changed with echocardiographic screening becoming the first-choice imaging modality for patients in a curative as well as a palliative setting.

\section{ii) Cardiac screening prior to hematopoietic stem cell transplantation}

Due to the high risk of life-threatening complications, haematopoietic stem cell transplantation (HSCT) is preserved for relatively young patients with adequate vital organ functions [II]. As part of the pre-HSCT work-up, all patients undergo screening at our cardio-oncology service. Patients that have received anthracycline-containing induction therapy prior to the planned HSCT are assumed to be at high risk for the development of anthracycline-induced cardiac dysfunction (AICD) and therefore, this patient group receives intensive cardiac screening during the first year after anthracyclines have been administered.

\section{iii) Long-term screening after hematological malignancies}

Patients with Hodgkin lymphoma (HL) treated with anthracyclines (regardless of dose if combined with mediastinal radiotherapy, or an equivalent dose of $\geq 300 \mathrm{mg} / \mathrm{m} 2$ doxorubicin) undergo echocardiographic examination every 5 years [I2]. In HL patients solely treated with mediastinal radiotherapy, echocardiography is performed once, I5 years after diagnosis. According to local policy, patients that have undergone an allogeneic stem cell transplantation are referred for echocardiographic screening 5 years after transplantation.

\section{iv) Cardiac complaints}

Besides the screening of patients at risk for (late) cardiovascular adverse events, patients with any cardiac complaints deemed to be possibly related to the cancer treatment are also referred. 


\section{Data collection}

Data was retrieved from the electronic health records including details on cancer diagnosis and -treatment and cumulative doxorubicin dose corrected for body surface area. For other anthracyclines, an equivalent doxorubicin dose was calculated using the following conversion factors: doxorubicin I; daunorubicin 0.83; epirubicin 0.67; idarubicin 5; mitoxantrone 4 [13]. Furthermore, pre-existing cardiovascular disease and -risk factors were determined. Diagnoses were based on the reporting by the treatment physicians, substantiated by relevant therapies or evidence from additional investigations such as laboratory or radiological analysis.

Outcomes measures included the occurrence of CTRCD, its clinical presentation and its effect on oncological treatment plans. Patients were diagnosed with CTRCD if they met one of the following two criteria: (I) an LVEF decline of $\geq$ IO percentage points below the lower limit of normal $(<53 \%)$ from baseline according to the definition described by Plana et al. [I4], or (2) an LVEF $<50 \%$ measured at $>$ I time-point in case a baseline LVEF measurement prior to treatment was absent. Other possible causes of an impaired LV function, such as ischemic heart disease, left bundle branch block, severe valvular heart disease, tachycardiomyopathy, or an impaired ventricular function during sepsis were evaluated to determine the likelihood of CTRCD. The New York Heart Association (NYHA) classification was used to evaluate clinical symptoms. Additional outcome measures included i) the occurrence of other cardiovascular adverse events (CVAE) that were deemed likely related to oncological treatment, and their effect on oncological treatment plans ii) major Adverse Cardiac Events (MACE), defined as hospitalization due to heart failure, or cardiac death and iii) all-cause mortality.

\section{Statistical analysis}

Continuous data are expressed as means and standard deviation or medians and interquartile ranges [IQR]. Categorical variables are expressed as frequency (percentages). Continuous data were compared using the independent Student's t-test or Mann-Whitney U and categorical data were tested using Chi-square or Fisher exact test as appropriate. A two-sided p-value $<0.05$ was considered significant. Statistical analyses were performed using SPSS Statistics, version 25 (IBM Corp., Armonk, NY, USA).

\section{Results}

Between April 2015 and September 2019, 800 patients (age $53.3 \pm$ I4.8 years, 51.6\% females) were evaluated at the cardio-oncology outpatient clinic (Figure I). The median follow-up at the cardio-oncology service was 5.0 [IQR O-I3.7] months, and general clinical follow-up information was available for a median time of I4.3 [IQR 5.5 - 28.0] months. The median number of visits to the cardio-oncology clinic per patient was 2 [IQR I-4]. One hundred-seven $(\mathrm{I} 3 \%)$ patients died during the planned follow-up period at the cardio-oncology service (oncological $n=96$; cardiac $n=3$; other $n=8$ ). 
Hematological malignancies were present in 518 (65\%) patients and $192(24 \%)$ patients had breast cancer (Figure I). 680 (85\%) patients received treatment with curative intent and most commonly this concerned first-line treatment. Chemotherapy was given to 760 (95\%) patients, of which 55I (73\%) received anthracyclines. The median cumulative doxorubicin dose was $240 \mathrm{mg} / \mathrm{m}^{2}$ [IQR 194-330]. Chemotherapy was combined with targeted- or immunotherapy in 297 (37\%) patients.

Most patients were referred for cardiac screening ( $\mathrm{n}=639 ; 80 \%)$, of which 497 (78\%) were seen prior or during treatment and I42 (22\%) received screening for long-term cardiovascular effects. Cardiac screening prior to stem cell transplantation comprised the largest patient group ( $\mathrm{n}=284 ; 35 \%)$, whereas $\mathrm{I} 22$ patients were referred for cardiac evaluation prior to potentially cardiotoxic chemotherapy.

At first consultation, coronary artery disease was the most common pre-existing cardiovascular comorbidity $(6.6 \%)$, followed by arrhythmia (6.3\%) and cardiomyopathy (5.6\%). Four in five patients (8I.9\%) had no previous history of cardiac disease and $296(37 \%)$ had no cardiovascular risk factor and 294 (37\%) had one cardiovascular risk factor (Figure I).

\section{Cardiovascular adverse events}

Of the 800 patients that visited the cardio-oncology service, $286(36 \%)$ patients experienced a CVAE during follow-up, of which CTRCD was diagnosed most frequently $(n=I 76 ; 22 \%)$ (Figure I). In I82 patients, CVAEs were detected during cancer treatment, resulting in treatment interruption in $7(4 \%)$ patients and treatment discontinuation in 30 ( $16 \%)$ patients. In IO4 patients, the adverse event was diagnosed after antineoplastic treatment was completed and therefore did not have any impact on further scheduled treatment

Arrhythmias were observed in 75 patients (9\%), and most commonly concerned atrial fibrillation- or flutter $(\mathrm{n}=56 ; 75 \%)$. In most patients, arrhythmias were diagnosed during infection $(n=30 ; 40 \%)$ or administration of systemic cancer treatment $(n=18 ; 24 \%)$. None of the oncological treatments had to be discontinued or interrupted due to arrhythmias, and no life-threatening arrhythmias were observed.

Forty-three patients were treated with agents associated with vasospasms (i.e. 5-fluorouracil, capecitabine) of which five (II.6\%) patients experienced chest pain. One patient developed cardiac ischemia of the anterior wall in the absence of significant coronary artery disease, leading to capecitabine discontinuation. The four other patients completed treatment without the occurrence of MACE.

\section{Cardiac screening in patients receiving Trastuzumab}

Eighty-eight patients treated with trastuzumab received 3-monthly echocardiographic screening. Additionally, I9 patients screening with MUGA scans by the treating oncologists were referred because of cardiac complaints ( $n=6$ during- and $n=13$ after treatment). Out of 88 patients that received trastuzumab with curative intent, CTRCD was diagnosed in 25 (28\%) after a median time of 5.9 [IQR 2.7-7.4] months after trastuzumab had been initiated. More than half of patients did not have any (NYHA I/IV; $\mathrm{n}=\mathrm{I} 4 ; 54 \%$ ) or only mild (NYHA 


\section{Cardio-Oncology Service}
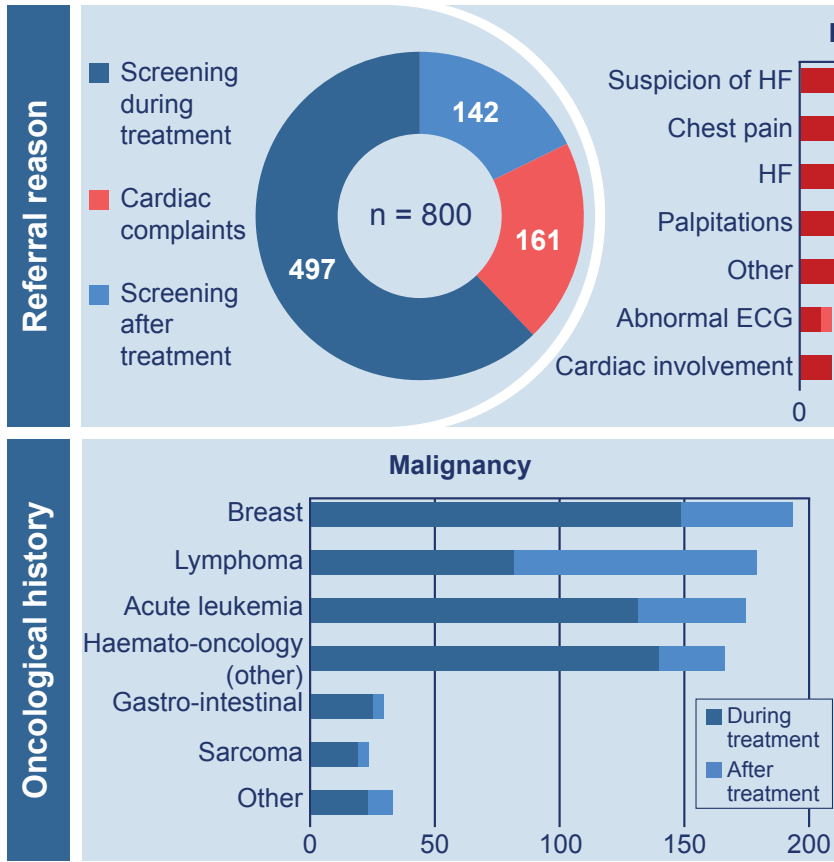

Referral for cardiac complaints
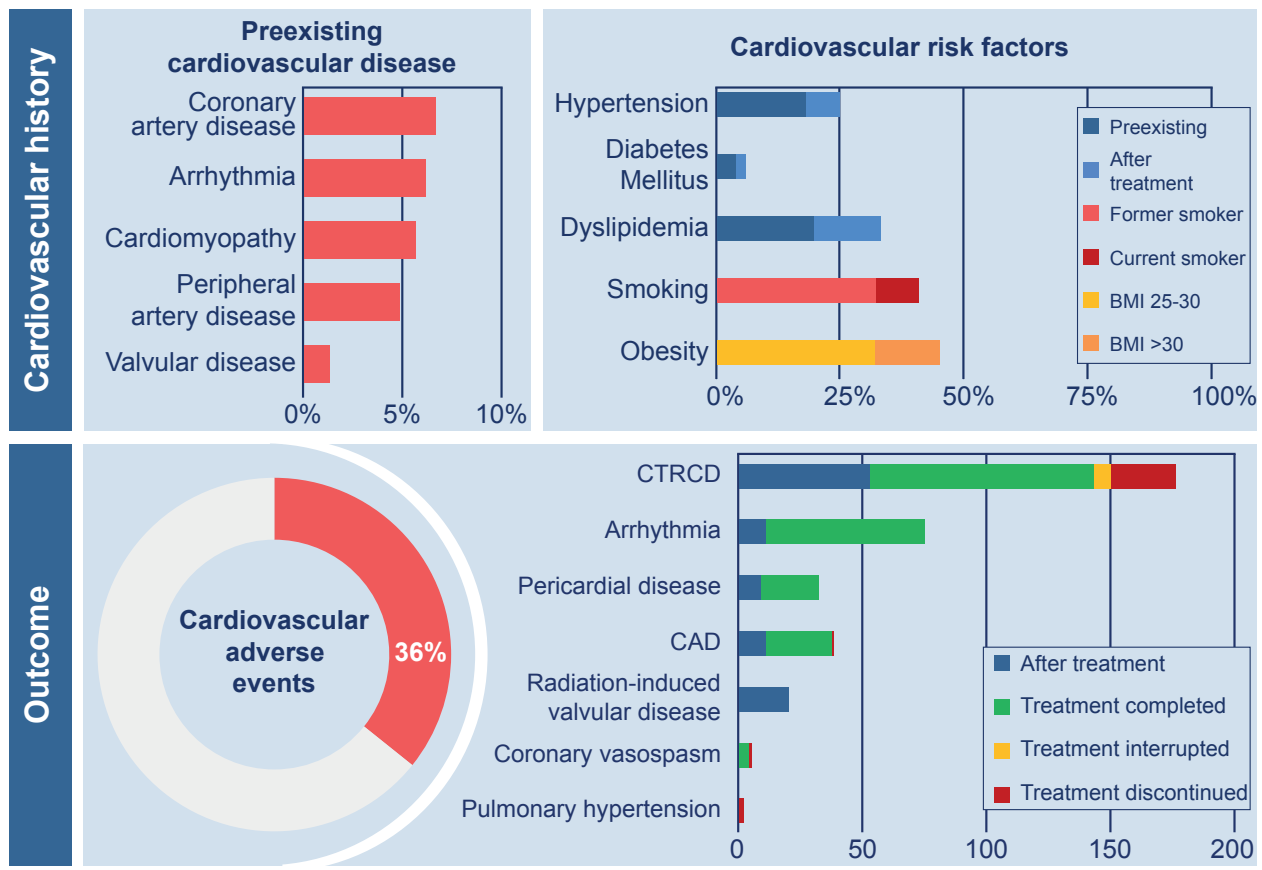

Figure I Overview of the patient population and outcomes of the specialized cardio-oncology service 
$\mathrm{II} / \mathrm{IV} ; \mathrm{n}=\mathrm{IO} ; 38 \%$ ) complaints. One patient presented with heart failure requiring hospitalization. Compared to patients without CTRCD, patients that were diagnosed with this complication were more likely to have received prior treatment with anthracyclines (Table I). Treatment was discontinued in 24 patients, and heart failure medication was initiated in I4 patients. I9 patients did not complete the planned treatment due to persistent LVEF impairment. During follow-up, I2 (67\%) of these patients eventually had an LVEF above the lower limit of normal.

Palliative treatment with trastuzumab was given to 19 patients with a median treatment duration of I4.7 (range 1.2-79) months. Trastuzumab discontinuation was predominantly caused by disease progression $(\mathrm{n}=\mathrm{IO} ; 53 \%)$, and in a lesser degree due to CTRCD ( $\mathrm{n}=6 ; 32 \%)$. The median time between trastuzumab initiation and occurrence of CTRCD was 4.I months, and was also observed as late as after 23.7 months. We observed no MACE in the patient group that received palliative treatment with trastuzumab.

\section{Cardiac screening prior to hematopoietic stem cell transplantation}

Cardiac screening was performed in 238 and 46 patients planned for an allogeneic- and autologous stem cell transplantation respectively. Of the 284 patients that received cardiac assessment prior to HSCT, 193 were treated with anthracyclines (Table 2). In this patient group, 66 (34\%) patients were diagnosed with CTRCD, after a median time of 6.4 [2.4-I6.6] months after initiation of anthracycline-containing therapy. 6I patients were diagnosed at the cardio-oncology service and had no- $(n=4 I ; 67 \%)$ or mild $(n=19 ; 31 \%)$ symptoms of heart failure. The other 5 patients developed symptoms of clinical heart failure after initiation of anthracycline-containing treatment and prior to the Ist cardiac screening. The mean LVEF at diagnosis was $44 \pm 5 \%$, and the majority of patients $(n=54 ; 82 \%)$ had a mildly impaired systolic function (i.e. LVEF $40-52 \%$ ). HSCT had to be cancelled in one patient due to severe heart failure. In the other patients, treatment did not need to be interrupted or -discontinued due to CTRCD. Heart failure medication was initiated in 45 (68\%) patients and, according to local policy, 2I patients were closely monitored without initiation of heart failure medication since they lacked cardiac complaints and the LVEF was $\geq 45 \%$ [7]. After a median follow-up of I2 [IQR 5-30.8] months, 33 (50\%) died. Death was oncology-related in $29(88 \%)$ patients, I (3\%) death was heart failure-related, and $3(9 \%)$ deaths were not related to oncologic- or cardiac disease.

The cumulative anthracycline dose was higher in the CTRCD group although this did not reach significance $\left(296 \pm \mathrm{I} 28 \mathrm{mg} / \mathrm{m}^{2}\right.$ vs $\left.26 \mathrm{I} \pm \mathrm{I} \mathrm{I} 2 \mathrm{mg} / \mathrm{m}^{2} ; \mathrm{p}=0.052\right)$. Apart from a higher prevalence of pre-existing abnormal cardiac function in the CTRCD group, we were unable to discriminate patients with- and without CTRCD based on patient-related risk factors and the cardiotoxicity risk score (Table 2).

\section{Long-term screening after hematological malignancies}

In total, I69 patients were referred by the hematology department for screening of longterm cardiovascular toxicity after oncological treatment. Hodgkin lymphoma $(n=58 ; 34 \%)$, non-Hodgkin lymphoma (NHL; $n=42 ; 25 \%)$ and acute leukemia $(n=44 ; 26 \%)$ were the most 
Table I Characteristics of patients that received curative treatment with trastuzumab. Differences between patients with- and without CTRCD are shown.

\begin{tabular}{|c|c|c|c|}
\hline & $\operatorname{CTRCD}(n=25)$ & No CTRCD $(n=63)$ & p-value \\
\hline Age at treatment (years) & $50.8($ II. .3$)$ & 53.I (I2.I) & 0.408 \\
\hline Treatment with anthracyclines & $20(80)$ & $3 I(50)$ & $0.010^{*}$ \\
\hline Left-sided radiotherapy & $9(36)$ & I3 (2I) & o.I44 \\
\hline Hypertension & $7(28)$ & I5 (24) & 0.712 \\
\hline Diabetes Mellitus & $\mathrm{o}(\mathrm{o})$ & I (2) & 0.523 \\
\hline \multicolumn{4}{|l|}{ Smoking } \\
\hline None & 15 & 38 & \multirow{3}{*}{0.870} \\
\hline Former & 7 & I8 & \\
\hline Current & 3 & 5 & \\
\hline Coronary artery disease & I (4) & $3(5)$ & 0.866 \\
\hline Pre-existing abnormal cardiac function ${ }^{\#}$ & $4(\mathrm{I} 6)$ & $4(6)$ & 0.163 \\
\hline Arrhythmia & $3(\mathrm{I} 2)$ & $4(6)$ & 0.389 \\
\hline \multicolumn{4}{|c|}{$\begin{array}{l}\text { Categorical variables are expressed as } \mathrm{n}(\%) \text { and continuous variables as mean (standard deviation); }{ }^{*} \mathrm{p} \text {-values }<0.05 \text { are } \\
\text { considered significant; \# functional and/or structural (e.g. ischemic-, non-compaction-, hypertrophic) cardiomyopathies; } \\
\text { CTRCD, cancer therapy-related cardiac dysfunction; }\end{array}$} \\
\hline
\end{tabular}

Table 2 Characteristics of patients that were treated with anthracyclines and received cardiac screening prior to HSCT. Differences between patients with- and without CTRCD are presented.

\begin{tabular}{|c|c|c|c|}
\hline & $\operatorname{CTRCD}(n=66)$ & No CTRCD (n=I27) & p-value \\
\hline \multicolumn{4}{|l|}{ Haematological diagnosis } \\
\hline Acute leukemia & $4 \mathrm{I}$ & 78 & \\
\hline Lymphoma & I5 & 25 & \\
\hline Multiple myeloma & 2 & I2 & \\
\hline Myelodysplastic syndrome & 3 & II & \\
\hline Other & 5 & I & \\
\hline Age at treatment (years) & $50.2(\mathrm{I} 4 . \mathrm{I})$ & $49.9(15.4)$ & 0.901 \\
\hline \multicolumn{4}{|l|}{ Gender } \\
\hline Female & $19(29)$ & $53(42)$ & \multirow[t]{2}{*}{0.078} \\
\hline Male & $47(7 \mathrm{I})$ & $74(58)$ & \\
\hline Cumulative anthracycline dose $\left(\mathrm{mg} / \mathrm{m}^{2}\right)$ & 296 (I28) & 26I (II2) & 0.052 \\
\hline Cardiotoxicity Risk Score & 5.0 (I.O) & $5.0(\mathrm{I} . \mathrm{I})$ & 0.928 \\
\hline Hypertension & I5 (23) & $26(20)$ & 0.349 \\
\hline Diabetes Mellitus & $6(9)$ & $5(4)$ & 0.143 \\
\hline \multicolumn{4}{|l|}{ Smoking } \\
\hline None & $35(54)$ & $66(55)$ & \multirow{3}{*}{0.169} \\
\hline Former & 2I (32) & $46(39)$ & \\
\hline Current & $9(\mathrm{I} 4)$ & $7(6)$ & \\
\hline Coronary artery disease & 7 (II) & $7(6)$ & 0.196 \\
\hline Pre-existing abnormal cardiac function ${ }^{*}$ & $5(8)$ & $2(2)$ & $0.034^{*}$ \\
\hline Arrhythmia & $5(8)$ & $4(3)$ & 0.167 \\
\hline \multicolumn{4}{|c|}{$\begin{array}{l}\text { Categorical variables are expressed as n (\%) and continuous variables as mean (standard deviation); * p-values }<0.05 \text { are con- } \\
\text { sidered significant; \# functional and/or structural (e.g. ischemic-, non-compaction-, hypertrophic) cardiomyopathies; HSCT, } \\
\text { hematopoietic stem cell transplantation; CTRCD, cancer therapy-related cardiac dysfunction; }\end{array}$} \\
\hline
\end{tabular}


prevalent malignancies.

Mediastinal radiation had been given in 46 patients, with a median dose of 37.8 [35-40] Gray. After a median time of 17.2 [9.7-22.8] years between radiation therapy and the evaluation, radiation-induced abnormalities were detected in $17(37 \%)$ patients (Figure 2). Valvular abnormalities, ranging from sclerosis to severe valvular disease, were observed in 14 (30\%) patients, after a median time of 22.8 [19.5-3I.0] years after radiotherapy (mean 39.6 \pm 2.4 Gray), and at a median age of 50.0 [IQR 43.2-59.6] years. The aortic valve was involved in 7 patients, 2 patients had mitral valve abnormalities and both left-sided valves were affected in 5 patients. One patient required aortic valve surgery. The other patients were monitored more frequently $(n=9)$ or, according to the BETER protocol [I2], at 5-yearly intervals $(n=4)$. Diagnostic tests for coronary artery disease were performed in 20 patients. Coronary artery disease requiring intervention was diagnosed in 8 patients with a mean age of 63.2 +/-8.7 years, and after a median time of 22.8 [19.5-3I.0] years after radiation therapy (Figure 2).

CTRCD was diagnosed in 38 (22\%) patients referred for long-term screening, after a median time of 7.9 [I.4-I6.6] years (Table 3). Anthracyclines were administered in 36 (95\%) patients, with a significant higher cumulative dose compared to patients without CTRCD $(3 \mathrm{I} 7 \pm \mathrm{I} 44 \mathrm{mg} / \mathrm{m} 2$ vs $246 \pm 93 \mathrm{mg} / \mathrm{m} 2 ; \mathrm{p}=0.04 \mathrm{I}) .33$ patients were diagnosed at the outpatient service, whereas 5 patients had their first contact when they were hospitalized due to heart failure. These 5 patients received further care at our cardio-oncology service. Heart failure medication was given to $30(79 \%)$ patients, while in 8 patients treatment was not initiated due to lack of cardiac complaints and an LVEF $\geq 45 \%$ [7]. During a median follow-up of 2.5 [IQR I.3-3.5] years after CTRCD diagnosis, one more patient was hospitalized due to heart failure and one patient died due to endocarditis.

\section{Discussion}

The main purpose of this study was to provide an overview of our cardio-oncology clinic, describe our first experiences and its clinical yield. The main findings are threefold: i) in our pre-selected population, CVAEs occurred in more than one-third (36\%) of patients ii) in $84 \%$ of patients in which CVAEs were diagnosed during treatment, cancer treatment could be completed, and iii) with serial cardiac assessment, CTRCD was frequently diagnosed and in the absence of monitoring would likely have not been detected as the majority of patients had no (55\%) or only mild (34\%) cardiac complaints.

\section{Cardiac screening in patients receiving trastuzumab}

The quarterly monitoring of breast cancer patients receiving one-year therapy with adjuvant trastuzumab resulted in a large number (28\%) of patients diagnosed with CTRCD. However, the high incidence is likely a reflection of a selection bias, as cardiac screening was coordinated by the treating oncologist after the initial launch of the cardio-oncology service. Back then, patients were only referred in case of abnormal cardiac findings.

To strive for optimal cancer treatment, the continuation of trastuzumab despite 


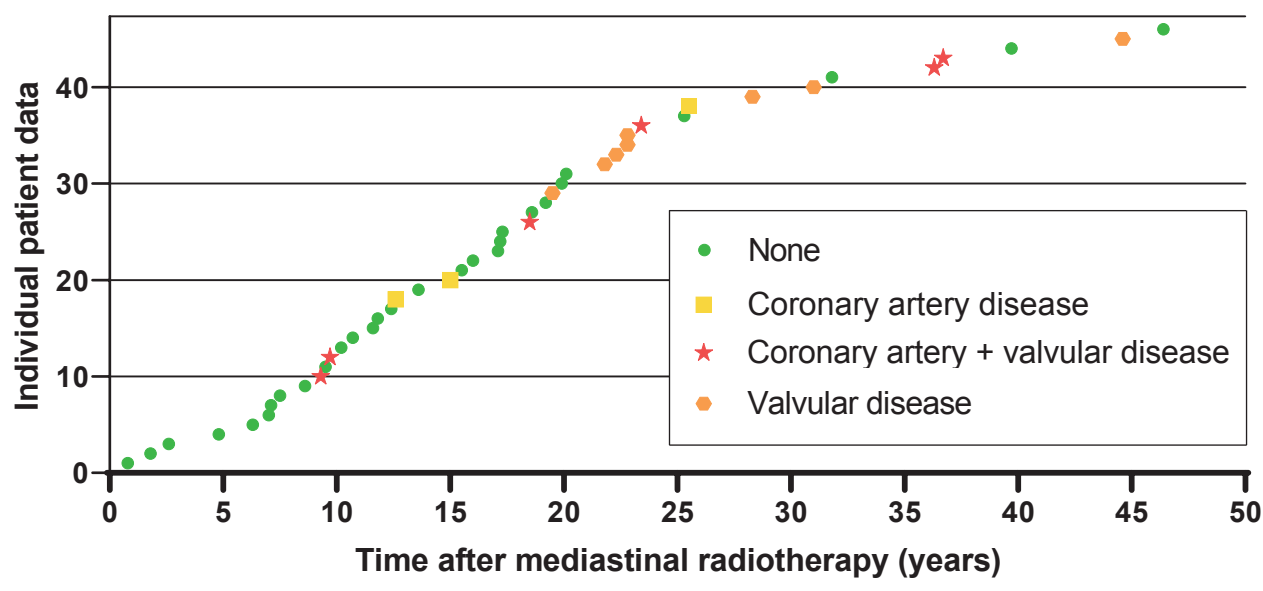

Figure 2 Radiation-induced cardiac disease in Hodgkin lymphoma patients receiving long-term cardiac screening. Individual patient data are presented in relation to time after mediastinal radiotherapy.

Table 3 Characteristics of patients undergoing long-term cardiac screening after hemato-oncological disease. Differences between patients with- and without CTRCD are presented.

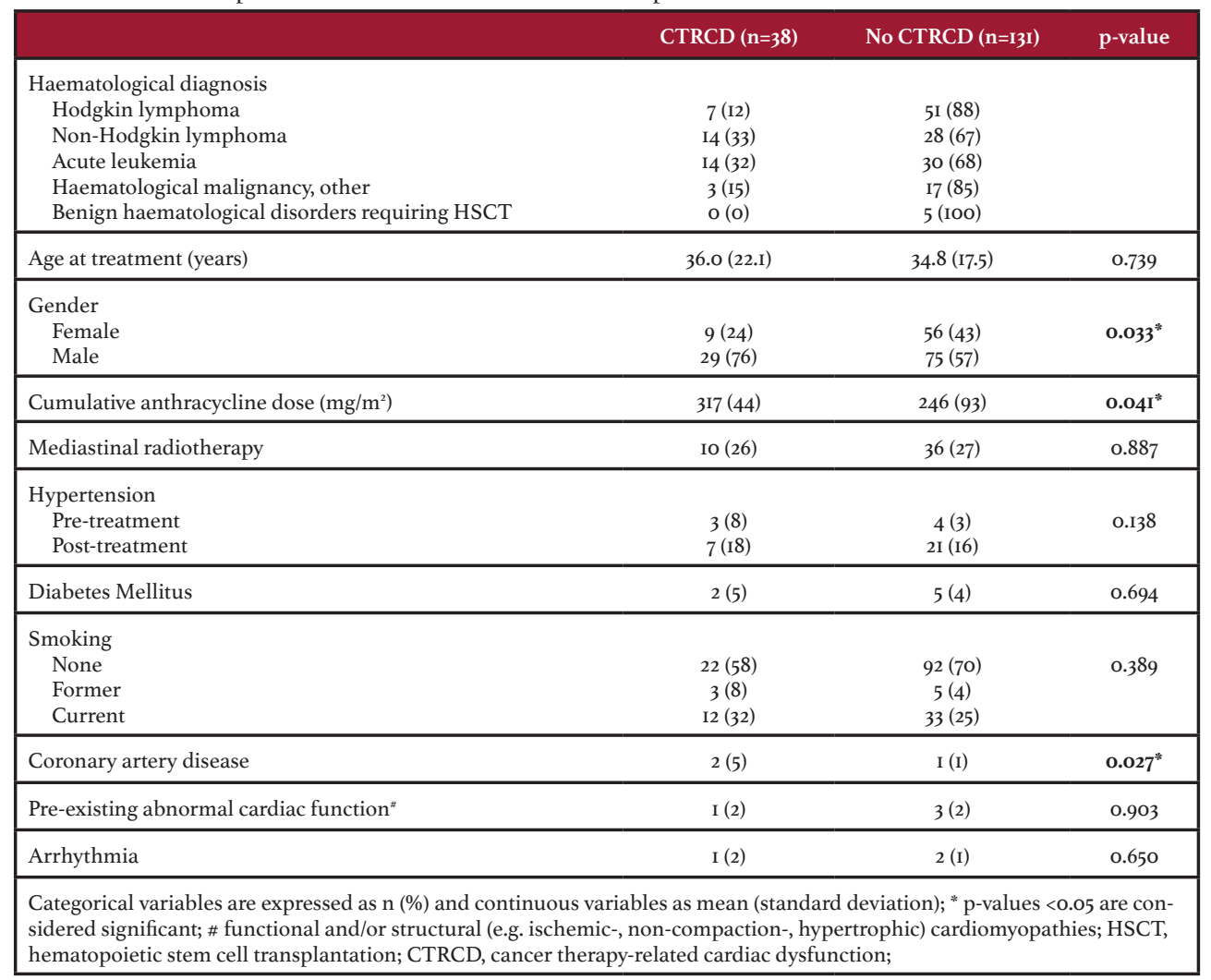


mild LV dysfunction has recently been evaluated in two pilot studies [15,I6]. After initiation of cardioprotective agents, $90 \%$ of patients successfully finished trastuzumab treatment without the occurrence of MACE. Thus, the continuation of trastuzumab under strict cardiac monitoring, together with cardioprotective medication, might be feasible in patients with mild LV dysfunction. As a result of these promising findings and in accordance with our oncologists, we have recently adopted this strategy in our cardio-oncology service protocol.

Trastuzumab continuation should also be considered in patients with mild (asymptomatic) LV dysfunction receiving palliative treatment with trastuzumab, as the benefits of trastuzumab treatment often outweigh the risk of developing serious cardiotoxicity. This is especially true for patients for whom no other antineoplastic treatment options are available.

\section{Cardiac screening prior to hematopoietic stem cell transplantation}

In patients that are diagnosed with CTRCD prior to HSCT, we handle a strategy to enable this life-saving intervention. Firstly, heart failure medication is initiated as soon as possible to prevent further deterioration of cardiac function. Secondly, haematologists are made aware of the risk of fluid overload in patients with impaired cardiac function. Thirdly, strict follow-up of cardiac function is mandatory for the long-term management of CTRCD. With this strategy, except for one patient, all patients were able to undergo an allogeneic stem cell transplantation.

Even though CTRCD did not have a major impact on oncological treatment in our patient population, also because further treatment regimens mostly did not include cardiotoxic agents, treating physicians should be aware of the consequences CTRCD can have on long-term outcomes. Asymptomatic LV dysfunction can eventually become symptomatic over time, for instance when other cardiac stressors such as hypertension or coronary artery disease lead to an additional decline of systolic function [I7]. On top of that, initiation of heart failure medication as soon as CTRCD is diagnosed gives the highest chance of cardiac recovery [I8] and therefore this therapy should only be interrupted if clinically necessary.

The cardiotoxicity risk score [8] (Chapter 2 Table 2) that is used to determine the need for screening at the cardio-oncological service and the intensity of follow-up, has not been validated yet. We explored the utility of this risk stratification tool in patients that were treated with anthracyclines and were referred for cardiac screening prior to allogeneic HSCT, since this patient population does not suffer from referral bias. We found that this tool was unable to discriminate between patients with- and without CTRCD. Also, even though female gender is considered as a risk factor in the current risk stratification tool, (non-significantly) more males were diagnosed with CTRCD. This unexpected low predictive value of the cardiotoxicity risk score underscores the need for validated risk prediction models. 


\section{Long-term cardiotoxicity after treatment for haematological malignancies}

Screening for long-term cardiotoxic effects was performed in HL-, NHL-, and acute leukemia survivors. HL patients are often treated with a combination of anthracycline-containing systemic treatment and (high-dose) thoracic radiotherapy [19], resulting in an increased risk for cardiovascular disease at long-term follow-up [20]. Therefore, survivorship programs for HL patients have included recommendations for cardiovascular risk management and the implementation of echocardiographic examinations [I2,2I,22].

The long-term screening and management of cardiovascular disease in NHL patients is, up to now, less clearly delineated. Since NHL treatment often contains high doses of anthracyclines, these patients are prone to develop CTRCD. A recent meta-analysis indeed found that, in case cardiac monitoring was performed after treatment, CTRCD was common (II.7\%) in NHL patients treated with the standard first-line regimen R-CHOP [23]. We also found a high number of NHL patients (33\%) suffering from CTRCD, of which most cases were detected during cardiac screening while being asymptomatic. It therefore seems reasonable to screen for CTRCD in NHL patients, since patients with asymptomatic CTRCD are at risk to develop clinical heart failure [24].

Cardiac screening in acute leukemia patients, for which treatment regularly consists of anthracycline-containing induction therapy, followed by HSCT, seems also useful. In our patient population, CTRCD was frequently observed during both short- (34\%) and long-term (33\%) screening. Currently, the management of treatment-related adverse effects in long-term HSCT survivors comprise yearly monitoring of cardiovascular risk factors [25], since the prevalence of cardiovascular risk factors are increased after HSCT [26]. Still, monitoring of cardiac function has not been adopted in this guideline, while CTRCD is a frequently observed side effect in our acute leukemia patient population, as well as in other studies $[27,28]$.

\section{Evaluation of current strategy and points of improvement}

Our cardio-oncology service handles a protocol that is based on recommendations from the European Society of Cardiology [3], European Association of Cardiovascular Imaging [I4] and management strategies from the Mayo Clinics [8]. Compared to other cardio-oncology services, differences are present regarding the patient population. For example, our patient population predominantly consists of patients suffering from haematological malignancies and breast cancer, whereas other services also provide care to patients with sarcomas or gastro-intestinal malignancies $[5,6]$.

Our care pathway does not yet include standardized follow-up strategies for patients treated with relatively new anticancer agents such as tyrosine kinase inhibitors (TKI) or immune checkpoint inhibitors (ICPI). TKIs are known for their risk of hypertension and thrombo-embolic complications [29], whereas cardiovascular toxicities from ICPIs are uncommon (<0.1\%) but can be potentially life-threatening [30]. If future guideline for management of cardiovascular complications of these new anticancer agents become available, we should consider to implement these recommendations in our pathway.

A non-validated risk stratification tool, based on retrospectively attributed risk 
risk factors, is used to identify patients prone to CTRCD that could benefit from cardiac screening [8]. Even though the number of patients with CTRCD in our population is high, the risk tool was unable to discriminate between patients with and without CTRCD which underscores the need to develop a risk score in a prospective cohort.

The screening of long-term CVAEs in hematological patients comprises one of the activities of our service. Though, a systematic approach for the long-term screening of all patients at risk for CVAEs is currently not adopted in our protocol. Patients in which CVAEs are detected will receive cardiac care according to personalized needs. For patients that remain free of CVAEs during the first year of screening, it is unknown whether CVAEs will become manifest later on. Long-term follow-up data is needed to establish if early screening is sufficient, or if monitoring during longer period of time is necessary. The longterm follow-up data of the current study will be collected in the Dutch registry 'ONCOR' (Netherlands Trial Register NL8064). This national registry has been launched to collect real-world data of patients seen at specialized cardio-oncology services. Together with outcomes of other cardio-oncology registries (3I-33), results can contribute to optimization of cardio-oncological care. Additionally, outcomes of the randomized TITAN-study can clarify whether, compared to usual care, intensive multidisciplinary team interventions are beneficial in the prevention of cardiotoxicity [34].

\section{Study limitations}

We handle a local referral policy, thereby aiming to provide care to patients at high risk for cardiovascular toxicities. In case patients that were assumed to be at low risk still experienced cardiovascular toxicities, these patients could be overlooked when these toxicities were treated by health care providers not related to the cardio-oncology service. This creates a certain bias of our study population. However, patients referred for cardiac screening prior to allogeneic HSCT did not suffer from referral bias, since all patients are referred to our service.

\section{Conclusion}

In conclusion, cardiovascular adverse events were commonly detected in the pre-selected patient population of our specialized cardio-oncology service, and in the majority of cases $(84 \%)$ these patients were able to complete cancer treatment. Cancer therapy-related cardiac dysfunction was regularly diagnosed in patients with no- or mild cardiac complaints, implicating that in the absence of active screening, this complication often will remain undetected. Long-term follow-up can provide further insight on the effects of specialized cardio-oncological care on both cardiac and oncological outcome. 


\section{References}

I de Moor JS, Mariotto AB, Parry C, et al. Cancer survivors in the United States: prevalence across the survivorship trajectory and implications for care. Cancer Epidemiol Biomarkers Prev.2013;22:56I-70.

2 Verdecchia A, Francisci S, Brenner H, et al. Recent cancer survival in Europe: a 2000-02 period analysis of EUROCARE-4 data. Lancet Oncol. 2007;8:784-96.

3 Zamorano JL, Lancellotti P, Rodriguez Muñoz D, et al. 2016 ESC Position Paper on cancer treatments and cardiovascular toxicity developed under the auspices of the ESC Committee for Practice Guidelines: The Task Force for cancer treatments and cardiovascular toxicity of the European Society of Cardiology (ESC). Eur Heart J. 2016;37:2768-80I.

4 Ewer MS, Ewer SM. Cardiotoxicity of anticancer treatments. Nat Rev Cardiol 2015; I2:620.

5 Kappel C, Rushton M, Johnson C, et al. Clinical experience of patients referred to a multidisciplinary cardio-oncology clinic: an observational cohort study. Curr Oncol. 2019;26(3):e322-e327.

6 Pareek N, Cevallos J, Moliner P, et al. Activity and outcomes of a cardio-oncology service in the United Kingdom-a five-year experience. Eur J Heart Fail. 20I8;20(I2):I72I-I73I.

7 Teske AJ, Linschoten M, Kamphuis JAM, et al. Cardio-oncology: an overview on outpatient management and future developments. Neth Heart J. 20I8;26(II):52I-532.

8 Herrmann J, Lerman A, Sandhu NP, Villarraga HR, Mulvagh SL, Kohli M. Evaluation and management of patients with heart disease and cancer: cardio-oncology. Mayo Clin Proc. 20I4;89:1287-306.

9 Trastuzumab package insert, version dated 29/II/20I8. Consulted at https://www.accessdata.fda.gov/ scripts/cder/daf/

Io Cardoso F, Kyriakides S, Ohno S, et al. Early breast cancer: ESMO Clinical Practice Guidelines for diagnosis, treatment and follow-up. Ann Oncol. 2019;30(I0):I674.

II Carreras E, Rambaldi A. Evaluation and Counseling of candidates. In: Carreras E, Dufour C, Mothy M, Kroger N. The EBMT Handbook. 7th edition. Springer open, 2019: 77-86

I2 Dekker N, van 't Veer MB, Aleman BM, van Leeuwen FE, Raemaekers JM. The BETER survivorship care initiative for Hodgkin lymphoma; tailored survivorship care for late effects of treatment. Ned Tijdschr Geneeskd. 2015;159:A9269.

I3 Shankar SM, Marina N, Hudson MM, et al. Monitoring for Cardiovascular Disease in Survivors of Childhood Cancer: Report From the Cardiovascular Disease Task Force of the Children's Oncology Group. Pediatrics. 2008;I2I(2):e387-96.

I4 Plana JC, Galderisi M, Barac A, et al. Expert Consensus for Multimodality Imaging Evaluation of Adult Patients during and after Cancer Therapy: A Report from the American Society of Echocardiography and the European Association of Cardiovascular Imaging. 20I4;15(I0):I063-93.

I5 Leong DP, Cosman T, Alhussein MM, et al. Safety of Continuing Trastuzumab Despite Mild cardiotoxicity: A Phase I Trial; JACC CardioOnc. 20I9;I(I)I-IO.

I6 Lynce F, Barac A, Geng X, et al. Prospective evaluation of the cardiac safety of HER2-targeted therapies in patients with HER2-positive breast cancer and compromised heart function: the SAFE-HEaRt study. Breast Cancer Res Treat. 2019;175(3):595-603.

I7 Eschenhagen T, Force T, Ewer Ms, et al. Cardiovascular side effects of cancer therapies: a position statement from the Heart Failure Association of the European Society of Cardiology. Eur J Heart Fail. 2OII;I3(I):I-IO.

I8 Cardinale D, Colombo A, Lamantia G, et al. Anthracycline-induced cardiomyopathy: clinical relevance and response to pharmacologic therapy. J Am Coll Cardiol. 2010;55:213-20.

I9 Eichenauer DA, Aleman BMP, André M, et al. Hodgkin lymphoma: ESMO Clinical Practice Guidelines for diagnosis, treatment and follow-up. Ann Oncol. 2018;29 Suppl 4:ivig-iv29.

20 van Nimwegen FA, Schaapveld M, Janus CP, et al. Cardiovascular disease after Hodgkin lymphoma treatment: 40-year disease risk. JAMA Intern Med. 20I5;I75(6):I007-I7.

2I Ng AK. Current survivorship recommendations for patients with Hodgkin lymphoma: focus on late effects. Hematology Am Soc Hematol Educ Program. 20I4;20I4(I):488-94.

22 National Comprehensive Cancer Network. NCCN Clinical Practice Guidelines in Oncology: Hodgkin Lymphoma. Version I.2020. Accessed on March I, 2020 at: http://www.nccn.org/professionals/ physician_gls/f_guidelines.asp. 
23 Linschoten M, Kamphuis JAM, van Rhenen A, et al. Cardiovascular adverse events in patients with non-Hodgkin lymphoma treated with first-line cyclophosphamide, doxorubicin, vincristine, and prednisone (CHOP) or CHOP with rituximab (R-CHOP): a systematic review and meta-analysis.

24 Lancet Haematol. 2020;7(4):e295-e308. Doi: Io.IOI6/S2352-3026(20)3003I-4. Echouffo-Tcheugui JB, Ergou S, Butler J, Yancy CW, Fonarow GC. Assessing the Risk of Progression From Asymptomatic Left Ventricular Dysfunction to Overt Heart Failure: A Systematic Overview and

25 Meta-Analysis. JACC Heart Fail. 20I6;4(4):237-48.

Majhail NS, Rizzo JD, Lee SJ, et al. Recommended screening and preventive practices for long-term

26 survivors after hematopoietic cell transplantation. Bone Marrow Transplant. 2012;47(3):337-4I. Armenian SH, Sun CL, Vase T, et al. Cardiovascular risk factors in hematopoietic cell transplantation

27 survivors: role in development of subsequent cardiovascular disease. Blood. 20I2;I20(23):4505-I2.

Mort MK, Sen JM, Morris AL, et al. Evaluation of cardiomyopathy in acute myeloid leukemia patients

28 treated with anthracyclines. J Oncol Pharm Pract. 2020;26(3):680-687

Kang Y, Assuncao BL, Denduluri S, et al. Symptomatic heart failure In acute leukemia patients treated

29 with anthracyclines. JACC CardioOnc 20I9;I(2):208-2I7

30 Moslehi JJ. Cardiovascular toxic effects of targeted cancer therapies. N Engl J Med. 2I06;375(I5):I457-67. Brahmer JR, Lacchetti C, Schneider BJ, et al. Management of Immune-Related Adverse Events in Patients Treated With Immune Checkpoint Inhibitor Therapy: American Society of Clinical Oncology 3I Clinical Practice Guideline. J Clin Oncol. 2018;36(I7):I7I4-I768.

Lancellotti P, Galderisi M, Donal E, et al. Protocol update and preliminary results of EACVI/HFA Cardiac Oncology Toxicity (COT) Registry of the European Society of Cardiology. ESC Heart Failure

32 20I7; 4(3): 312-3I8.

Clinicaltrias.gov [Internet]. Bethesda (MD): National Library of Medicin(US). Identifier NCTo3882580, Reporting, Evaluating, Preventing and Treating the Cardiotoxicity Induced by Anticancer Drugs During a Specific Cardio-oncology Consult and Follow up in Routine Care (UNICO); March 20, 2019

33 [Cited April 8, 2020]. Available from: https://www.clinicaltrials.gov/ct2/show/NCTo3882580

Clinicaltrias.gov [Internet]. Bethesda (MD): National Library of Medicin (US). Identifier NCTo2039622, Cardiovascular Toxicity Induced by Antitumoral Drugs: Risk Assessment and Early Diagnosis. CARDIOTOX Registry; January I7, 2014 [Cited April 8, 2020]. Available from: https://www.

34 clinicaltrials.gov/ct2/show/NCT02039622

Pituskin E, Haykowsky M, McNeely M, Mackey J, Chua N, Paterson I. Rationale and design of the multidisciplinary team IntervenTion in cArdio-oNcology study (TITAN). BMC Cancer. 20I6;I6:733. 
Chapter 7

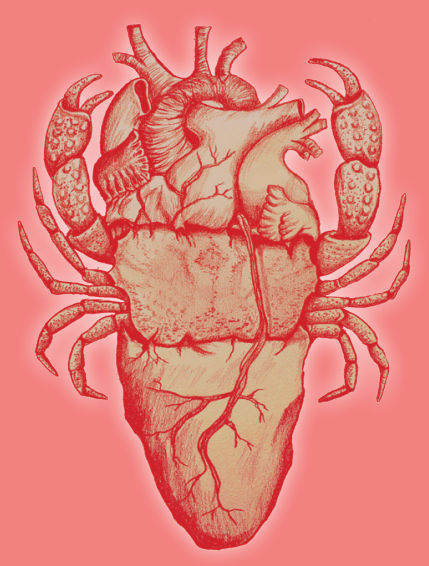




\title{
ONCOR \\ Design of the Dutch Cardio-Oncology Registry
}

\author{
Accepted for publication in Netherlands Heart Journal
}

\author{
JAM Kamphuis ${ }^{1}$, M Linschoten ${ }^{1}$, MJ Cramer ${ }^{1}$, \\ F Alsemgeest ${ }^{2}$, DJW van Kessel ${ }^{3}, \mathrm{~K}$ Urgel $^{3}, \mathrm{MC} \mathrm{Post}^{\mathrm{t}, 3}$, \\ OC Manintveld ${ }^{4}$, HC Hassing ${ }^{4}$, C Liesting ${ }^{5}$, AJ Wardeh ${ }^{6}$, \\ EGM Olde Bijvank ${ }^{6}$, J Schaap ${ }^{7}, \mathrm{M} \mathrm{Stevense}^{8}$, \\ PA Doevendans ${ }^{\mathrm{I}, 910}$, FW Asselbergs ${ }^{\mathrm{I}, \mathrm{IO}, \mathrm{II}}, \mathrm{AJ}$ Teske $^{\mathrm{I}}$
}

I Department of Cardiology, Division of Heart and Lungs, University Medical Centre Utrecht, University of Utrecht, Utrecht, The Netherlands

2 Department of Cardiology, St Jansdal Hospital, Harderwijk, The Netherlands

3 Department of Cardiology, St Antonius Hospital, Nieuwegein, The Netherlands

4 Department of Cardiology, Erasmus MC, Rotterdam, The Netherlands

5 Department of Cardiology, Albert Schweitzer Hospital, Dordrecht, The Netherlands

6 Department of Cardiology, Haaglanden Medical Center, The Hague, the Netherlands

7 Department of Cardiology, Amphia Hospital, Breda, The Netherlands

8 Department of Internal Medicine, Amphia Hospital, Breda, The Netherlands

9 Central Military Hospital, Utrecht, The Netherlands

Io Netherlands Heart Institute, Utrecht, The Netherlands

II Health Data Research UK, Institute of Health Informatics and Institute of Cardiovascular Science, Faculty

of Population Health Sciences, University College London, London, UK 


\begin{abstract}
Background The relative new subspecialty 'cardio-oncology' was established to meet the growing demand of an interdisciplinary approach for the management of cancer therapy-related cardiovascular adverse events. In recent years, specialized cardio-oncology services have been implemented worldwide, which all strive to improve cardiovascular health of cancer patients. However, limited data is currently available on the outcomes and experiences of these specialized services and optimal strategies for cardio-oncological care have not been established.
\end{abstract}

Aim The ONCOR registry has been created for prospective data collection- and evaluation of cardio-oncological care in daily-practice

Methods Dutch hospitals using a standardized cardio-oncology care pathway are included in this national, multicentre, observational cohort study. All patients visiting these cardio-oncology services are eligible for study inclusion. Data collection at baseline consists of (planned) cancer treatment and the cardiovascular risk profile, which are used to estimate the cardiotoxic risk. Information regarding (non-)invasive tests are collected during the time period patients receive cardio-oncological care. Outcome data consist of the occurrence of cardiovascular complications, major adverse cardiac events, and the impact of these events on the oncological treatment.

Discussion Outcomes of the ONCOR registry can contribute in providing more insight into the incidence of cancer therapy-related cardiovascular complications. The registry facilitates research on mechanisms of cardiovascular complications, diagnosis, prognosis and treatment strategies and provides a platform for future (interventional) studies. Centres with cardio-oncology services interested in contributing to the ONCOR registry are hereby invited to participate. 


\section{Introduction}

Improvements in the early detection and treatment of cancer has over the last decades lead to increasing numbers of cancer survivors worldwide [I,2]. Furthermore, the prognosis of patients treated in a palliative setting continues to improve and several malignancies are evolving into chronic conditions. With these advances, the prevention and management of short- and long-term treatment-related side effects is gaining importance.

Cardiovascular toxicities are among the most frequent unintended side effects of cancer treatment and can manifest in many forms including cancer therapy-related cardiac dysfunction (CTRCD), arrhythmias, valvular-, pericardial- and coronary artery disease (CAD) $[3,4]$. Compared to the general population, cancer survivors are at increased risk for all of these cardiovascular diseases [5], and in addition have a 2-6 times higher risk of cardiovascular mortality [6]. With the growing awareness of these complications among healthcare professionals treating cancer patients and -survivors and a demand for an interdisciplinary approach for their management, a new discipline termed 'cardio-oncology' has emerged since the late ' 90 [7]. The overarching aims of this subspecialty are the optimization of cardiovascular health of cancer patients with pre-existent cardiovascular disease to enable the initiation of the antineoplastic treatment with the most optimal benefit-risk ratio, improve cancer treatment tolerability, manage- and prevent cardiovascular complications.

Over the years, the focus of the field of cardio-oncology has shifted from treatment of cardiovascular complications, towards preventive strategies and early detection of these complications by implementation of cardio-oncology services across many centres across the world $[8,9]$. In line with these developments, the European Society of Cardiology (ESC) released a position paper on cancer treatments and cardiovascular toxicity in 20I6, which provides an overview of cardiovascular monitoring and decision-making in patients treated with potentially cardiotoxic cancer treatment [4]. However, evidence-based clinical guidelines on cardio-oncological care are currently lacking and the recommendations in the position paper are all based on expert opinion(s). Albeit cardio-oncology services all strive to improve cardiovascular health of cancer patients, different clinical approaches are handled according to local need and limited data is available on the outcomes and experiences of these specialized services [IO,II]. Optimal strategies for cardio-oncological care are therefore still unknown. Across the Netherlands, several cardio-oncology services have been established that provide care according to a standardized care pathway [I2]. To gain insight into the outcomes of patients visiting these specialized services, we have set up a national registry that collects data of daily-practice cardio-oncological care. 


\section{Methods}

Study design and setting

In April 20I5, a standardized cardio-oncology care pathway was developed through a collaboration between the Department of Cardiology, Haematology, Oncology, and Radiology at the University Medical Centre Utrecht (UMCU), Utrecht, the Netherlands [I2]. Various other Dutch hospitals have adopted this care pathway and set up cardio-oncology services as well in recent years, resulting in a large number of patients receiving standardized care across the Netherlands. The ONCOR registry is a national, multicentre observational cohort study, conducted at hospitals that provide care according to this pathway [I2]. The study is performed in collaboration with the Netherlands Heart Institute (NHI), Utrecht, the Netherlands.

\section{Study population}

All adult patients that receive(d) anticancer treatment and visited a cardio-oncology service are eligible to participate. Written information about ONCOR is provided by their treating physician at this service. Patients interested to participate are requested to provide written informed consent for the use of clinical data. Additionally, patients are asked for permission to be approached for future (interventional) studies.

The patient population seen across the cardio-oncology services largely consists of three subgroups (I) patients monitored for cardiovascular complications whilst undergoing treatment with potential cardiotoxic agents (2) patients screened for long-term cardiovascular complications and (3) patients with cardiac complaints possibly related to their oncological treatment. For the first group, the main focus lies on the early detection of CTRCD in patients deemed at (high) risk for this complication. The cardiotoxicity risk score (CRS)[13] is utilized for CTRCD risk stratification. The CRS is based on treatment-related risk factors, e.g. the antineoplastic agents, exposure to chest radiation etc. and patient-related risk factors such as age, sex, and the presence of cardiovascular risk factors and -diseases [I3] (Figure I). Based on the estimated incidence of CTRCD associated with the various antineoplastic agents, the treatment-related risk is subdivided into high- $(\geq \mathrm{IO} \%)$, intermediate- (5-IO\%), low (I-5\%), and rare $(<\mathrm{I} \%)$ (Figure I; Chapter 2 Supplemental File I). Currently, patients with a CRS of $\geq 4$ have an indication for referral to the cardio-oncology outpatient service to receive serial echocardiographic assessments during cancer treatment. Four is handled as a cut-off value to allocate resources to those patients most likely to benefit from cardio-oncological care. There are currently no risk stratification tools available for other cardiovascular complications and referral is at the discretion of the treating oncologist.

Screening for late cardiotoxic effects is performed in patients that received treatment with anthracyclines and/or mediastinal radiotherapy. For example, (non-)Hodgkin patients are monitored according to the BETER protocol [I4]. According to this protocol, cardiac screening is performed every 5 years if a patient was treated with an equivalent dose of doxorubicin $\geq 300 \mathrm{mg} / \mathrm{m}^{2}$ or $<300 \mathrm{mg} / \mathrm{m}^{2}$ if treatment was combined with mediastinal radiotherapy. Adult survivors of pediatric malignancies do not receive standard care at 


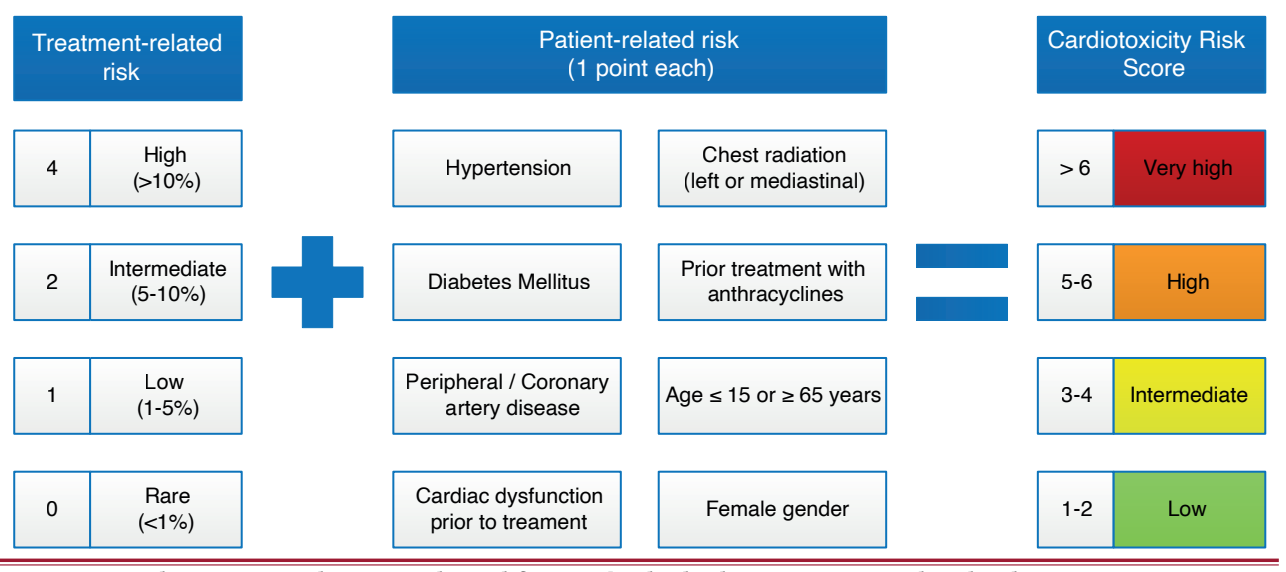

Figure I Cardiotoxicity Risk Score, adapted from [I3]. The highest treatment-related risk score (e. g. 4, 2, I or o) is used for calculation of the score. Risk scores of individual anticancer agents and -regimens are provided in supplemental file I

the cardio-oncology service, but receive cardiovascular screening according to the LATER protocol of the Dutch Childhood Oncology Group [15]. Patients with abnormal cardiovascular findings during LATER screening are referred to the cardio-oncology service for further management.

\section{Ethics and dissemination}

The registry follows the Code of Conduct for the Use of Data in Health Research. Inclusion of patients is exempted from the Medical Research Involving Human Subjects Act (WMO) as per judgement of the Medical Ethics Committee (METC 18-639/C, Utrecht, the Netherlands). Participating centres require approval from the local institutional ethical committee and a consortium agreement form needs to be signed which includes specifications on obligations, liability, confidentiality and data protection amongst others. The ONCOR registry is registered at the Netherlands Trial Registry, project NL8064 (www.trialregister.nl).

\section{Objectives of the ONCOR registry}

- $\quad$ Register the occurrence of cardiovascular complications classified according to the ESC Position Paper on cancer treatments and cardiovascular toxicity [4] (Figure 2)

- $\quad$ Register the incidence of Major Adverse Cardiovascular Events (MACE), defined as i) cardiac hospitalization (categorized by heart failure, acute coronary syndrome, arrhythmia, or 'other') ii) cardiac death iii) implantation of ICD or CRT-D/CRT-P devices or iv) (need for) cardiac mechanical support or orthotopic heart transplantation

- Facilitate research on mechanisms of cardiovascular complications, risk factors, diagnostic, prognostic and therapeutic strategies

- $\quad$ Provide a future platform for registry-based randomized controlled trials (RRCT) 


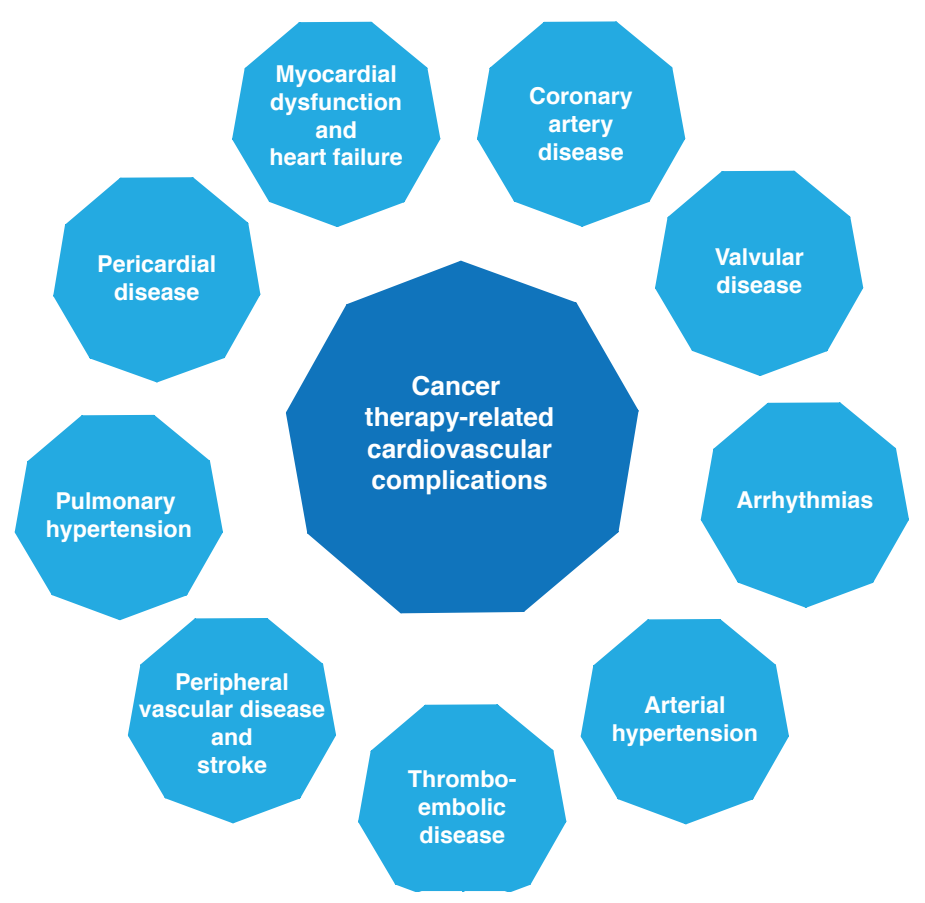

Figure 2 Cancer therapy-related cardiovascular complications

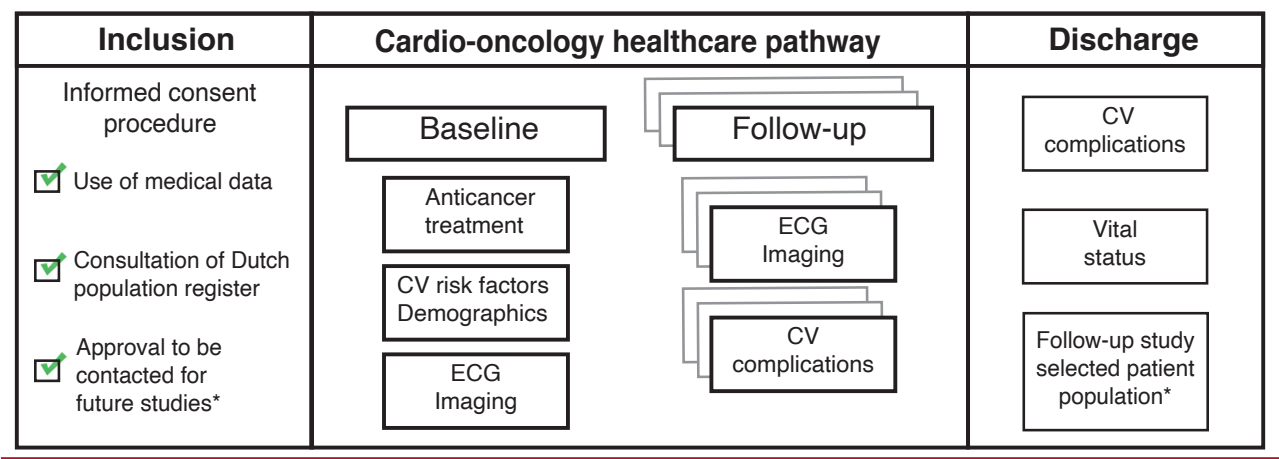

Figure 3 Informed consent procedure and data collection

\section{Data collection and-management}

Standardized data collection instruments are used for the registration of information collected during routine care (Figure 3). Uniform data collection is achieved by a Standard Operating Procedure (SOP) handbook, providing definitions of medical terms and instructions on how questions should be answered. For parties interested in participating in ONCOR, the data collection instruments, data dictionary and SOP handbook are available upon request. All data is handled confidentially and in accordance with Dutch privacy laws. Data are collected in an online REDCap database [I6], which handles an audit trail 
that registers data access, -entries and -changes. The database is hosted by the NHI Durrer Center, which provides IT-support, security and data protection. Entry of study data is performed by healthcare professionals involved in the cardio-oncology services at the participating centres. In each centre, a local coordinator is responsible for supervision of data entry. The data of each participating centre is stored in a separate data access group, which are only accessible for study members part of that centre and the national study coordinators.

\section{Data sharing}

Local centres are free to use data from their own centre for research purposes. When involved researchers want to use data from multiple participating centres, a data request must be submitted to the ONCOR Steering Committee. This Steering Committee is formed by the local coordinators from the participating centres. For each data request, local coordinators can decide on whether data from their centre may be used.

\section{Timeline}

The care pathway provides a standardized follow-up schedule for patients undergoing cancer treatment with potential cardiotoxic agents that are monitored for CTRCD. Typically, the follow-up visits of patients with active treatment lasts up to I year after completion of treatment with potentially (highly) cardiotoxic agents, as CTRCD can be detected within this time frame in a majority of patients susceptible to develop this complication [I7]. The frequency of follow-up visits is dependent on the patient-specific risk score and therefore varies between patients. Depending on the clinical course, the follow-up intensity and duration can be adjusted at the discretion of the treating physician. When patients are discharged from the cardio-oncology service, the occurrence of cardiovascular events are derived from the electronic health record (EHR). Information regarding follow-up will be requested in case patients received further care in another centre. The vital status is verified in the EHR or in the Dutch population register in case patients are lost to follow-up.

\section{Baseline assessment and clinical visits during follow-up}

The data collection instrument at baseline consists of information on the (planned) oncological treatment, including type of regimen, number of cycles, cumulative anthracycline dose and radiotherapy plans. Additionally, cardiovascular risk factors are registered. With these variables, the CRS is automatically calculated in REDCap. During the first- and standardized follow-up visits, clinical information on the presence of cardiac complaints and outcomes of physical examination, as well as results from electrocardiograms, echocardiograms and cardiac MRI, can be registered.

\section{Echocardiography and other cardiovascular investigations}

Serial cardiac assessment is done by using echocardiography, since this imaging technique is considered to be the most feasible imaging modality in cardio-oncological practice [I8]. Echocardiographic examinations are performed according to a standard protocol. The 
echocardiographic assessment includes analysis of cardiac dimensions- and function (including deformation imaging). LVEF measurements are preferably performed by 3D-measurements or, if not available, by the 2D biplane (modified Simpson's) algorithm or an estimation of the left ventricular function. Valvular analysis is performed at baseline and during long-term follow-up after thoracic radiotherapy. During follow-up in the Ist year after therapy, an analysis of the valves is performed upon indication (e.g. heart murmur) or when abnormalities were detected at baseline echocardiography. All echocardiographic outcomes, as well as the echo vendor can be registered within REDCap. Apart from the investigations part of the standardized cardio-oncologic follow-up, other additional investigations are performed upon indication at the discretion of the treating physician. Outcomes of these test results, such as analysis of CAD, Holter reports, genetic counseling and laboratory analysis, can be registered in the database. Since the value and optimal timing of measuring biomarkers has not yet been established, cardiac biomarkers such as troponin and (NT-pro)BNP are only measured at discretion of the treating physician and not yet part of the cardio-oncology care pathway [I2,I9].

\section{Cardiovascular complications}

During each clinical visit, the occurrence of cardiovascular complications is evaluated. Nine predefined categories of cardiovascular complications are used to register side effects in a uniform way (Figure 2). If a complication is diagnosed, information regarding the modalities and tests upon which the diagnosis is based is requested in REDCap. Furthermore, the impact of the cardiovascular complication on oncological treatment is registered.

\section{First results}

As of I2-02-202O, the ONCOR registry contains II42 individual patient records (Table I). Patients with breast cancer (39\%) and hematological malignancies (50\%) constitute the largest patient group. Cardiac follow-up after initiation of cancer treatment was available in I038 patients, with a median follow-up time of 2 I.6 [interquartile range I0.2 - 60.3] months. During this time period 378 cardiovascular complications were registered, of which CTRCD was the most common $(\mathrm{n}=2 \mathrm{20})$.

\section{Discussion}

Cardio-oncology is a subspecialty in its infancy and currently there is a gap in knowledge regarding the added value of cardio-oncology services. One of the largest challenges that lies ahead of health care professionals active in this field will be the identification of patients that will benefit from referral to these services prior to the initiation of cancer treatment, considering the large number of cancer patients and the accompanying restraints of resources at most cardiology departments. In each individual patient, the risk of cardiovascular complications must be weighed off against the oncological prognosis. This requires close collaboration between cardiologists and (haemato)oncologists. Patient groups likely 
Table I First results of the ONCOR registry

\begin{tabular}{|c|c|}
\hline \multicolumn{2}{|c|}{ Demographics } \\
\hline Number & II42 \\
\hline Age treatment & $50.8 \pm \mathrm{I} 6.8$ \\
\hline Age Ist consultation & $54.6 \pm \mathrm{I} 4.2$ \\
\hline Number of females & $700(6 \mathrm{I} \%)$ \\
\hline \multicolumn{2}{|c|}{ Malignancy } \\
\hline Breast cancer & $445(39 \%)$ \\
\hline Lymphoma & $2 \mathrm{I} 4(\mathrm{I} 9 \%)$ \\
\hline Acute leukemia & $\mathrm{I} 77(\mathrm{I} 6 \%)$ \\
\hline Hemato-oncology (other) & $\mathrm{I} 76(\mathrm{I} 5 \%)$ \\
\hline Other & $\mathrm{I} 30(\mathrm{II} \%)$ \\
\hline \multicolumn{2}{|c|}{ Referral reason } \\
\hline Screening during treatment & $76 \mathrm{I}(67 \%)$ \\
\hline Screening after treatment & I84 (I6\%) \\
\hline Cardiac complaints & $\mathrm{I} 97(\mathrm{I} 7 \%)$ \\
\hline \multicolumn{2}{|c|}{ Cancer therapy-related cardiovascular complications } \\
\hline Myocardial dysfunction and heart failure & 206 \\
\hline Coronary artery disease (including coronary vasospasm) & 27 \\
\hline Valvular disease & 22 \\
\hline Arrhythmias & 74 \\
\hline Arterial hypertension & 7 \\
\hline Thrombo-embolic disease & 4 \\
\hline Peripheral vascular disease and stroke & 2 \\
\hline Pulmonary hypertension & 2 \\
\hline Pericardial disease & 34 \\
\hline
\end{tabular}

to benefit most from active monitoring have a moderate to good oncological prognosis with a high life-time risk of developing cardiovascular complications such as patients with (non)-Hodgkin lymphoma's and breast cancer [5,6]. On the long-term, with the establishment of the ONCOR registry, we hope to contribute in providing more insight into the incidence of cardiovascular complications, the impact of early detection- and treatment of cardiovascular complications on patient prognosis.

\section{Limitations}

One of the limitations of ONCOR is that the present care pathway uses a risk stratification tool to pre-select patients who are currently believed to benefit from cardiac monitoring 
for CTRCD. This risk score predominantly depends on the cardiotoxic risk of the administered anticancer agent. Due to gaps in literature concerning the cardiotoxic profile of several agents [20], this can result in both and under- and overestimation of the true risk. In addition, the risk score has not yet been prospectively validated and introduces selection bias. Patients who are currently deemed having a low or negligible risk of CTRCD according to this risk score may develop cardiovascular complications that remain undetected in the subclinical phase in the absence of screening. Moreover, besides CTRCD, risk factors for the development of other cardiovascular complications are largely unknown.

Even though the cardio-oncological care is standardized as much as possible, the collected data originates from daily clinical practice from multiple centres, and therefore could induce issues such as vendor dependency and inter- and intra-observer variability. These issues will be taken into account when the echocardiographic data is analysed for multicentre studies.

\section{Future perspectives}

The current care pathway is predominantly focused on monitoring patients receiving treatment with anthracyclines and/or trastuzumab. However, the number of targeted therapies is rapidly expanding, of which many are associated with specific cardiovascular complications including myocarditis [21,22]. The need for management of targeted therapy-related cardiovascular complication will increase and implementation of management strategies in the care pathway is necessary. Additionally, the currently used risk stratification of anticancer agents can be revised when new insights on cardiotoxic profiles are available.

Expansion of ONCOR with for example biobanks can aid in the identification of biomarkers and genetic factors and thereby contribute to enhancing etiologic, diagnostic and prognostic knowledge of unintended cardiovascular effects of anticancer treatment. The establishment of an imaging biobank can help to correct for issues such as inter- and intra-observer variability. Blinded images can be examined by an independent cardiologist, using vendor neutral analysis software. Furthermore, the registry can be used for pragmatic trials to determine optimal screening methods and treatment strategies [23].

\section{Conclusion}

The ONCOR registry offers a platform for the collection of data from cardio-oncological care provided according to a standardized care pathway in various Dutch hospitals. The registry can aid in improving cardiac surveillance strategies and thereby contribute to improvement of cardiovascular health in the growing population of cancer patients and -survivors. Centres with a cardio-oncology service or centres that aim to launch such a service in the future interested to participate in the ONCOR registry are hereby invited to participate. 


\section{References}

I Miller KD, Nogueira L, Mariotto AB, et al. Cancer treatment and survivorship statistics, 2019. CA Cancer J Clin. 2019;69(5):363-385

2 Verdecchia A, Francisci S, Brenner H, et al. Recent cancer survival in Europe: a 2000-02 period analysis of EUROCARE-4 data. Lancet Oncol. 2007;8(9):784-96

3 Naaktgeboren WR, Linschoten M, de Graeff A, et al. Long-term cardiovascular health in adult cancer survivors. Maturitas. 20I7; I05:37-45

4 Zamorano JL, Lancellotti P, Rodriguez Muñoz D, et al. 2016 ESC Position Paper on cancer treatments and cardiovascular toxicity developed under the auspices of the ESC Committee for Practice Guidelines: The Task Force for cancer treatments and cardiovascular toxicity of the European Society of Cardiology (ESC). Eur Heart J. 2016;37(36):2768-28oI.

5 Strongman H, Gadd S, Matthews A, et al. Medium and long-term risks of specific cardiovascular diseases in survivors of 20 adult cancers: a population-based cohort study using multiple linked UK electronic health records databases. Lancet. 2019;394(IO203):IO4I-IO54

6 Sturgeon KM, Deng L, Bluethmann SM, Zhou S, Trifiletti DM, Jiang C, Kelly SP, Zaorsky NG. A population-based study of cardiovascular disease mortality risk in US cancer patients. Eur Heart J. 2019;40(48):3889-3897

7 Cardinale D. A new frontier: cardio-oncology. Cardiologia. 199;4I(9):887-89I.

8 Lancelotti P, Suter TM, López-Fernández, et al. Cardio-Oncology Services: rationale, organization, and implementation. Eur Heart J. 2019;40(22):1756-I763

9 Snipelisky D, Park JY, Lerman A, et al. How to develop a cardio-oncology clinic. Heart Fail Clin. 20I7;13(2):347-359

Io Pareek N, Cevallos J, Moliner P, et al. Activity and outcomes of a cardio-oncology service in the United Kingdom-a five-year experience. Eur J Heart Fail. 20I8;20(I2):I72I-I73I.

II Kappel C, Rushton M, Johnson C, et al. Clinical experience of patients referred to a multidisciplinary cardio-oncology clinic: an observational cohort study. Curr Oncol. 2019;26(3): e322-e327

I2 Teske AJ, Linschoten M, Kamphuis JAM, et al. Cardio-oncology: an overview on outpatient management and future developments. Neth Heart J. 2018;26(II):52I-532

I3 Herrmann J, Lerman A, Sandhu NP, Villarraga HR, Mulvagh SL, Kohli M. Evaluation and management of patients with heart disease and cancer: cardio-oncology. Mayo Clin Proc. 2014;89(9):1287-306.

I4 Dekker N, van 't Veer MB, Aleman BM, et al. The BETER survivorship care initiative for Hodgkin lymphoma; a tailored survivorshipcare for late effects of treatment. Ned Tijdschr Geneeskd.2015;159: A9269.

I5 SKION LATER. Richtlijn follow-up na kinderkanker-meer dan 5 jaar na diagnose. Aanbevelingen I603-20I0. Den Haag/Amsterdam, The Netherlands SKION 201.

I6 Harris PA, Taylor R, Thielke R, Payne J, Gonzalez N, Conde JG. Research electronic data capture (REDCap) - a metadata-driven methodology and workflow process for providing translational research informatics support. J Biomed Inform. 2009;42: 377-8I.

I7 Cardinale D, colombo A, Bacchiani G, et al. Early detection of anthracycline cardiotoxicity and improvement with heart failure therapy. Circulation. 2015;I3I:I98I-I988.

I8 Plana JC, Galderisi M, Barac A, et al. Expert Consensus for Multimodality Imaging Evaluation of Adult Patients during and after Cancer Therapy: A Report from the American Society of Echocardiography and the European Association of Cardiovascular Imaging. 20I4;15(I0):I063-93.

I9 Curigliano G, Lenihan D, Fradley M, et al. Management of cardiac disease in cancer patients throughout oncological treatment: ESMO consensus recommendations. Ann Oncol.2020;31(2):171-I90.

20 20. Kamphuis JAM, Linschoten M, Cramer MJM, et al. Cancer therapy-related cardiac dysfunction of non-anthracycline chemotherapeutics: What's the evidence? JACC CardioOncology. 2019;I(2):280-90.

2I Moslehi JJ. Cardiovascular toxic effects of targeted cancer therapies. N Engl J Med. 2016;375(I5):I457I467.

22 Zaha VG, Meijers WC, Moslehi J. Cardio-Immuno-Oncology. Circulation. 2020;I4I(2):87-89

23 James S, Rao SV, Granger CB. Registry-based randomized clinical trials - a new clinical trial paradigm. Nat Rev Cardiol. 2015;12(5):312-6. 


\section{Chapter 8}

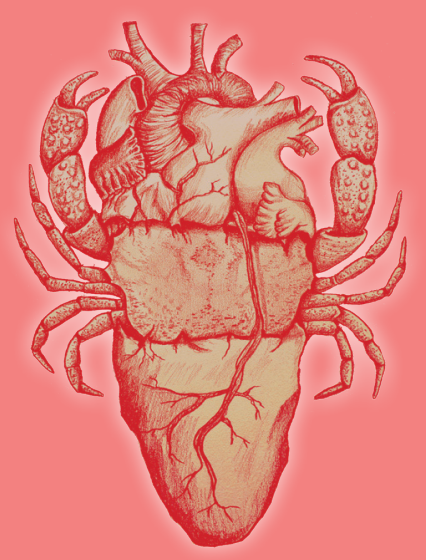


Summary and Future perspectives 


\section{Stepping stones to improve cardio-oncological care}

Since the establishment of the subspecialty 'cardio-oncology' in the I990s, considerable progress has been made regarding the knowledge of cancer therapy-induced cardiovascular toxicity. Whereas shortly after the introduction of cardio-oncology, the focus was on treatment of short- and long-term cardiovascular adverse events of cancer treatment, this focus has gradually shifted towards early detection and prevention of these side effects. To meet the growing need for a structured clinical approach to perform cardiac screening of patients at risk for cardiovascular adverse events, numerous specialized cardio-oncology clinics have been established worldwide. Thus far, these clinics use local protocols, since international, evidence-based guidelines on the performance of cardio-oncological care are still lacking.

In 20I5, a collaboration between the departments of oncology, haematology and cardiology at the University Medical Center Utrecht resulted in the establishment of a specialized cardio-oncology care pathway, which is delineated in Chapter 2. This pathway provides a systematic approach for the management of cancer therapy-related cardiovascular toxicities, and in particular for the early detection and treatment of cancer therapy-related cardiac dysfunction (CTRCD). Yet, several questions regarding the optimal execution of cardio-oncological care, such as risk stratification, screening methods and -intervals, treatment, and prevention of cancer therapy-induced cardiotoxicity are still unanswered. In this thesis, evaluation of the abovementioned issues have been studied by means of i) evaluating the assumptions regarding cardiotoxicity of anticancer agents and -regimens (Chapter 3, 4) ii) an in-depth analysis of the clinical presentation and management of anthracycline-induced cardiac dysfunction (Chapter 5) and iii) evaluating the outcomes of a specialized cardio-oncology care pathway (Chapter 6 and 7).

\section{Risk stratification for cancer therapy-related cardiac dysfunction}

An important aim of the cardio-oncology service is the early detection of CTRCD, since it is assumed that early detection and early treatment initiation provides the best outcome regarding recovery of cardiac function [I]. Mostly, CTRCD does not immediately lead to symptoms of heart failure (HF) [2], and therefore, active screening for CTRCD is essential for early identification. Screening for CTRCD should be offered to high risk patients. This risk is determined by both treatment- and patient-related factors.

\section{Treatment-related risk factors}

The risk of CTRCD is predominantly determined by the oncological treatment, of which anthracyclines and trastuzumab are specifically known for their high risk of causing CTRCD (Chapter I). Nonetheless, the oncological armamentarium consists of a variety o $\mathrm{f}$ chemotherapeutic agents, of which several are also considered to carry a high risk of CTRCD. In Chapter 3, we performed an in-depth review of 5 chemotherapeutic agents (i.e. mitomycin-C, vincristine, clofarabine, ifosfamide and cyclophosphamide) which are considered to be highly cardiotoxic [3-6]. The main message of this review is that commonly used 
incidence rates of CTRCD of these 5 chemotherapeutics should be interpreted with caution, since several sources on which these rates were based are questionable. The first limitation is that the cardiotoxic effects were seldomly investigated in patients receiving monotherapy. Instead, patients frequently received concomitant treatment with other cardiotoxic drugs such as anthracyclines. The combined treatment makes it impossible to determine the true cardiotoxic effects of the specific agent. The second limitation is that incidence rates were not based on results of a systematic search, but predominantly consisted of a modest selection of retrospective studies. The majority of these studies were published shortly after introduction of the chemotherapeutic agents. During this time period, the diagnosis of CTRCD was generally based on clinical signs of HF. Contrasting earlier evidence, a drop in left ventricular ejection fraction (LVEF), regardless of the presence of HF symptoms is currently also considered as CTRCD. Since monitoring of cardiac function was not routinely performed, only severe cases of CTRCD were observed, while subclinical CTRCD remained unnoticed. Thirdly, several studies handled the term 'cardiotoxicity' to define CTRCD, whereas other studies used cardiotoxicity as an umbrella term for all cardiovascular adverse events such as arrhythmias or coronary artery disease.

The main purpose of our 'primer in cardio-oncology' was to highlight the pitfalls in the interpretation of known cardiotoxicity data. Even though establishing more reliable cardiotoxicity data was beyond of the scope of the review, it offers several recommendations to improve future research on cardiotoxicity of anticancer agents and regimens. First of all, the performance of a systematic search that includes prospective clinical trials can avoid selection bias. Also, clinical trials nowadays use standardized grading systems to report adverse events, such as the National Cancer Institute Common Terminology Criteria for Adverse Events (CTCAE). Outcomes of adverse events using these standardized grading systems, can be combined from multiple studies to obtain cardiotoxicity incidence rates from a large patient population. Secondly, it is important to know which definition was used for the diagnosis of CTRCD in studies and trials. Whereas historically the diagnosis was based on clinical symptoms, the current definition also includes a decrease in LVEF, regardless of the presence of HF symptoms. Thirdly, to establish the true incidence of CTRCD of specific agents, the occurrence of CTRCD should be monitored in patients receiving monotherapy. However, patients are seldomly treated with monotherapy and for clinical use, it is more valuable to determine the risk of CTRCD of commonly used anticancer regimens.

In Chapter 4, we implemented the recommendations that were derived from Chapter 3 by performing a systematic search on clinical trials in which patients were treated with CHOP or R-CHOP. This regimen, which contains rituximab (R), cyclophosphamide $(\mathrm{C})$, doxorubicin $(\mathrm{H})$, vincristine $(\mathrm{O})$ and prednisolone $(\mathrm{P})$, has been used as first-line treatment for non-Hodgkin lymphoma (NHL) since I976 [7] and is currently still the first-line treatment for patients with diffuse large B-cell lymphoma [8]. (R-)CHOP contains two agents that are associated with CTRCD, namely doxorubicin and cyclophosphamide. We evaluated the occurrence of grade 3 (severe) + grade 4 (life-threatening) cardiovascular adverse events (CVAE), as well as the occurrence of HF. Due to the use of a standardized 
reporting system of adverse events, we were able to combine outcomes of 77 studies (I4,35I patients) and derived a pooled proportion of grade $3+4$ CVAEs of $2.35 \%$. A pooled proportion of $4.62 \%$ was derived from 38 studies $(5,936$ patients) that reported the occurrence of HF.

The systematic analysis of clinical studies in patients treated with R-CHOP already provides a more reliable impression of the cardiotoxic profile of this specific regimen. Nonetheless, we experienced that the reporting of CVAEs in oncological studies is frequently suboptimal. For example, many studies reported the number of CVAEs as a whole, without specifying the nature of the CVAEs, such as arrhythmias, coronary artery disease, or HF. This resulted in significantly less studies that could be used for the calculation of the occurrence of HF (38 out of 77). Also, grade I (mild) and grade (2) CVAEs were only reported in a limited number of studies $(n=23)$. Furthermore, it was often unclear when, and how cardiac screening was performed. Future clinical trials within the field of oncology should consider to present a more precise description of CVAEs, since different cardiovascular side effects ask for specific approaches of screening, prevention, treatment, and modifications in oncological treatment. Also, studies should report if, when and how cardiac screening was performed. This information can aid the interpretation of reported side effects, since adverse events may not induce symptoms and therefore could be missed in the absence of cardiac screening. The incorporation of these advises in oncology trials can contribute to a better insight into the cardiotoxic profile of anticancer treatments.

\section{Risk stratification models}

Several risk models have been developed to assist in the prediction of CTRCD. These models are focusing on specific patient populations such as breast cancer patients [9], childhood cancer survivors [IO-I2], acute leukemia patients [I3], or patients receiving specific treatment, e.g. trastuzumab [14] or anthracyclines [I5]. The factor 'age' has been found to be of influence in many of the abovementioned risk stratification models [9-II,I3-I5]. Both very young, as well as elderly patients seems to be at particular risk for the development of CTRCD. Other risk factors that have been adopted by various models are the well-known cardiovascular risk factors such as hypertension and diabetes mellitus, as well as preexisting cardiovascular disease such as coronary artery disease, decreased left ventricular function, and atrial fibrillation.

At our cardio-oncology service, the Cardiotoxicity Risk Score (CRS) [3] has been adopted to determine which patients have a high a priori risk to develop CTRCD and therefore could benefit from intensive screening at the cardio-oncology service. The CRS is applicable to multiple types of malignancies, since it is based on treatment- and patient-related factors. Within this thesis we were able to confirm the risk of contributing effect of some of the items included in the CRS. Particularly, the R-CHOP meta-analysis (Chapter 4), from which the variables 'age' and 'gender' could be analyzed, found that increasing age and female gender were explicitly associated with an increased risk of severe cardiovascular adverse events and more patients with CTRCD had a preexisting abnormal cardiac function, compared to patients without CTRCD (Chapter 6). 
Even though the CRS is a practical tool for risk assessment, it has not been valida ted properly yet. In Chapter 6, we explored the value of patient-related factors of the CRS in a group of patients who were all, regardless of the CRS, referred for cardiac screening prior to stem cell transplantation. Based on patient-related factors, the CRS was unable to discriminate between patients with- and without CTRCD. This risk tool therefore seems suboptimal to select patients at high risk for CTRCD. Further research is warranted to compose a risk stratification tool that is suitable for use in clinical practice.

\section{Treatment and prevention of anthracycline-induced cardiac dysfunction}

Anthracycline-induced cardiac dysfunction (AICD) has long been considered a severe form of cardiomyopathy, often refractory to standard HF treatment. This is based on older studies that included small sample sizes or even single case reports [I6-I8], in which patients were treated with high cumulative doses of anthracyclines [I6]. Furthermore, HF treatment in that era sometimes only consisted of the administration of digoxin and diuretics [I6-I9]. More recent studies, that used modern HF treatment with ACE inhibitors and beta blockers have found more promising effects on the improvement of cardiac function [20-2I]. A study of Cardinale et al. (2010) was the first to describe an effect of timing of HF treatment initiation on cardiac improvement in patients with anthracycline-induced cardiac dysfunction [I]. When HF treatment was initiated early after anticancer therapy, more patients had improvement of cardiac function. The authors also emphasized the importance of early recognition of AICD, as none of the patients diagnosed $>6$ months after cancer therapy completion had complete recovery of LVEF [I].

Up to now, the optimal treatment of anthracycline-induced cardiac dysfunction remains unclear. The European Society of Cardiology and European Society of Medical Oncology have published recommendations for the management of cardiovascular disease in cancer patients [5,22]. Initiation of ACE inhibitors and betablockers is recommended in case of symptomatic cardiac dysfunction, and these medications are also recommended [5] or to be considered [22] in case of asymptomatic cardiac dysfunction. The cardio-oncology care pathway that we developed uses comparable strategies regarding the treatment of symptomatic cardiac dysfunction. Though, patients with an asymptomatic LVEF of 45-53\% will be monitored and HF treatment will only be initiated in case patients develop symptomatic HF or further decrease in LVEF to $<45 \%$. Whether the strategy of watchful waiting in patients with mild, asymptomatic cardiac dysfunction is justified, should be evaluated in a long-term follow-up study.

In Chapter 5, we evaluated the echocardiographic phenotype and response to $\mathrm{HF}$ treatment in patients who were diagnosed with anthracycline-induced cardiac dysfunction at the cardio-oncology service. One of the findings was that the majority of patients were diagnosed while having a mildly impaired, non-dilated cardiomyopathy in the absence of HF symptoms. This finding is in contrast to the initial assumption that anthracyclines induce a cardiomyopathy with severely impaired function [I6]. The discrepancy might be explained by the lower cumulative dose of anthracyclines patients receive nowadays. Also, 
patients at the cardio-oncology service were diagnosed during screening, instead of presentation with HF symptoms [I6]. Patients diagnosed late after anthracycline treatment were less likely to have (partial) recovery of cardiac function in response to HF treatment, as was also seen in the study of Cardinale et al. (20IO) [I]. This led to the assumption that the timing of discovery of cardiac dysfunction appears to be of particular importance for the treatment of this complication. However, this should be reevaluated in future prospective studies, since Chapter 5 represents observational data derived from routine clinical care.

\section{Preventive strategies}

In the past years, several studies have investigated the impact of neurohormonal therapies, such as ACE inhibitors and betablockers, on the prevention of CTRCD.

A multicenter randomized trial studied the effect of enalapril on troponin elevation, as well as the incidence of cardiac dysfunction in patients treated with anthracyclines [23]. Patients were randomized to a 'prevention group' in which all patients received enalapril during anthracycline treatment, or to a 'troponin-triggered group' in which enalapril was initiated in case of troponin elevation. The number of patients experiencing troponin elevations was comparable between the two treatment strategies (prevention group: 23\%; troponin-triggered group: $26 \%$; $=0.50$ ), suggesting that enalapril does not prevent cardiomyocyte necrosis. As the incidence of cardiac dysfunction was low in both groups (prevention group: $1.5 \%$; troponin-triggered group: $0.7 \%$; $=$ =..00), the authors propose that enalapril is beneficial in the prevention of cardiac dysfunction, probably due to inhibition of compensatory mechanisms that normally induce adverse remodeling and progression towards cardiac dysfunction.

A recent meta-analysis evaluated the effect of preventive neurohormonal treatment on the change in LVEF from baseline to the end of the trial [24]. The study included I7 randomized clinical trials, in which $74 \%$ of patients were treated with anthracyclines. The pooled analysis revealed a more favorable outcome for patients treated with neurohormonal therapy, compared with patients treated with placebo. Nevertheless, this estimated difference in LVEF decline was small (i.e. 3.96\%), and could fall within the inter-test variability of the LVEF measurement. Furthermore, the clinical implication of such a small benefit in percentage of LVEF preservation remains undetermined. Since the results of the analysis were accompanied by both significant heterogeneity as well as a significant publication bias, the authors advocated the need for larger trials on cardioprotective strategies. For now, cardioprotective therapy through neurohormonal agents is not standardly recommended in patients treated with anthracycline-containing cancer therapy.

Dexrazoxane is an effective cardioprotective agent of AICD. The first study showed an attenuation of patients developing symptomatic HF after anthracycline therapy [25]. Additionally, compared to controls, patients treated with dexrazoxane had less decline in LVEF, and no- or minimal anthracycline-associated myocardial lesions on histopathological examination [25]. A meta-analysis of dexrazoxane, including 5 clinical trials with a total of 643 patients who were treated with anthracyclines (i.e. doxorubicin, epirubicin), showed beneficial effects regarding the prevention of $\mathrm{HF}$ 
(risk ratio 0.29, 95\% confidence interval 0.20 - 0.4I, p<0.00ooI) [26]. Importantly, this meta-analysis did not find differences between the dexrazoxane and control group regarding response to anticancer treatment, overall survival and progression-free survival. The majority of these trials have included patients with metastatic breast cancer. Therefore, the current use is not applicable to all patients receiving anthracyclines, but restricted to women with metastatic breast cancer who have received a prior cumulative doxorubicin dose of $300 \mathrm{mg} / \mathrm{m}^{2}$ and who will continue to receive doxorubicin therapy to maintain tumor control [27]. Due to lack of data, the efficacy of dexrazoxane in patients with malignancies other than breast cancer, or who receive curative anthracycline-containing treatment remains unknown.

Theoretically, dexrazoxane could be a valuable agent for the prevention of anthracycline-induced cardiotoxicity. Of particular interest in this light are patients with low cancer mortality and high risk of anthracycline-induced cardiac dysfunction, such as patients with breast cancer or non-Hodgkin lymphoma (NHL). As was discussed in Chapter 4, R-CHOP is the first-line regimen in patients with diffuse large B-cell lymphoma (DLBCL), a subtype of NHL [8]. From the studies that performed cardiac screening after therapy, we derived a proportion of patients with HF of II.72\% after R-CHOP treatment. Patients with DLBCL have a relatively good prognosis, with a 5-year survival rates of $64 \%$ [28]. Therefore, future research on the efficacy of dexrazoxane could be particularly interesting in this patient population.

\section{The yield of specialized cardio-oncology services}

Chapter 6 gives an overview of the experiences of providing specialized cardio-oncological care according to the pathway delineated in Chapter 2. The majority of patients seen at the cardio-oncology service were referred for screening of CTRCD. As a result of intensive cardiac screening during- and in the $\mathrm{I}^{\text {st }}$ year after cancer treatment, CTRCD could be detected frequently. In general, the phenotype of this adverse event was mild, asymptomatic, and did not affect oncological treatment. Yet, due to the relative short follow-up period of this study, the impact of early detection of CTRCD on long-term cardiac outcomes remained undefined. The outcomes of long-term follow-up of patients visiting the cardio-oncology services are thus crucial to evaluate the impact of cardio-oncological care. Therefore, the Dutch cardio-oncology registry 'ONCOR' has been established to provide a platform for data collection of patients receiving daily-practice cardio-oncological care (Chapter 7). This registry aims to collect long-term follow-up data, leading to the possibility to evaluate the disease course of CTRCD. The effect of HF treatment initiation on recovery of cardiac function, as well as the policy of not initiating HF treatment in asymptomatic CTRCD patients with LVEF between $45-53 \%$ can be evaluated. Additionally, it is interesting to observe whether CTRCD still occurs when it has not been observed in the $\mathrm{I}^{\text {st }}$ year of intensive cardiac screening. If so, modifications to the duration of cardiac screening of the care pathway need to be applied.

The collection of real-world data in the ONCOR registry has both strengths and limitations. The strength of data collection on daily-practice cardiac care, is that it does 
not exclude patients with cardiac comorbidities. In clinical oncology trials, patients with cardiac comorbidities are frequently excluded beforehand (Chapter 4), leading to restricted knowledge of CVAEs in these patients. On the other hand, the patient population within the registry only consists of patients seen at cardio-oncology services. These patients have been selected with the use of a risk stratification tool which has not been validated yet. Patients who are assumed to be at low risk can still suffer from CTRCD, but remain undetected in the absence of cardiac screening. Therefore, the ONCOR registry has limited use for the development of a risk stratification tool. To optimize risk stratification models, future research should focus on both patients who are currently assumed to be at low- and high risk for the development of CTRCD.

Worldwide, cardio-oncology services are established to optimize cardiovascular health of cancer patients. Even though the intentions to execute specialized cardio-oncological care are well-meant, the added value of these services is not seen by all health care professionals. This is illustrated by the outcomes of an international survey that evaluated the knowledge and opinion regarding cardio-oncological care of both cardiologists and oncologist [29]. The majority of cardiologists encouraged regular cardiac screening of (asymptomatic) cancer patients, whereas half of the oncologists would only consult a cardiologist in case patients develop cardiotoxicity. Also, $88 \%$ of cardiologists think cardio-oncology clinics contribute to improved prognosis of cancer patients and only $46 \%$ of the oncologists agree to this statement. Very few (8\%) oncologists strongly agreed that cardiologists were skilled to manage cardiovascular complications, whereas at least $40 \%$ of the cardiologists thought they were very suitable to manage cardiotoxicity.

The real value of specialized cardio-oncological care still needs to be proven. This can be done by, for example, performing a randomized controlled trial in which patients receive standard $v s$ cardio-oncological care, including outcome measures of both oncological and cardiac outcomes. This trial design is currently implemented in the TITAN-study [30]. Outcomes of this trial will shed more light on the value of cardio-oncological care.

\section{Concluding remarks}

The field of cardio-oncology has made great progress in the last years, and specialized cardio-oncological care is more and more implemented in hospitals all over the world. To improve the field of clinical cardio-oncology, there is need for amelioration of knowledge of cardiotoxicity profiles of cancer agents- and regimens. Furthermore, the optimal risk stratification model for clinical practice has not been established yet. Long-term follow-up of patients receiving cardio-oncological care need to demonstrate whether this intervention indeed results in improved cardiac outcome later on. 


\section{References}

I Cardinale D, Colombo A, Lamantia G, et al. Anthracycline-induced cardiomyopathy: clinical relevance and response to pharmacologic therapy. J Am Coll Cardiol. 2010;55:213-220.

2 Cardinale D, Colombo A, Bacchiani G, et al. Early detection of anthracycline cardiotoxicity and improvement with heart failure therapy. Circulation. 2015;131:198I-I988.

3 Herrmann J, Lerman A, Sandhu NP, Villarraga HR, Mulvagh SL, Kohli M. Evaluation and management of patients with heart disease and cancer: cardio-oncology. Mayo Clin Proc. 20I4;89:I287-I306.

4 Pai V, Nahata M. Cardiotoxicity of chemotherapeutic agents. Incidence, treatment and prevention. Drug Saf 2000;22:263-302.

5 Zamorano JL, Lancellotti P, Rodriguez Muñoz D, et al. 2016 ESC Position Paper on cancer treatments and cardiovascular toxicity developed under the auspices of the ESC Committee for Practice Guidelines: The Task Force for cancer treatments and cardiovascular toxicity of the European Society of Cardiology (ESC) Eur Heart J. 2016;37:2768-28oI.

6 Yeh E, Bickford C. Cardiovascular complications of cancer therapy: Incidence, pathogenesis, diagnosis and management. J Am Coll Cardiol 2009;53:223I-2247.

7 McKelvey EM, Gottlieb JA, Wilson HE, et al. Hydroxyldaunomycin (adriamycin) combination chemotherapy in malignant lymphoma. Cancer 1976; 38: I484-93.

8 Tilly H, Gomes da Silva M, Vitolo U, et al. Diffuse large B-cell lymphoma (DLBCL): ESMO Clinical Practice Guidelines for diagnosis, treatment and follow-up. Ann Oncol. 2015;26 Suppl 5:vIr6-25.

9 Abdel-Qadir H, Thavendiranathan P, Austin PC, et al. Development and validation of a multivariable prediction model for major adverse cardiovascular events after early stage breast cancer: a populationbased cohort study. Eur Heart J. 2019 Dec 21;40(48):3913-3920.

Io Chow EJ, Chen Y, Kremer LC, et al. Individual Prediction of Heart Failure Among Childhood Cancer Survivors. J Clin Oncol. 2014;33:394-402.

II Chen Y, Chow EJ, Oeffinger KC, et al. Traditional Cardiovascular Risk factors and individual prediction of cardiovascular events in childhood cancer survivors. J Natl Cancer Inst. 2020;II2(3):256-265.

I2 Van der Pal HJ, van Dalen EC, van Delden E, et al. High risk of symptomatic cardiac events in childhood cancer survivors. J Clin Oncol. 2012;30(I3):I429-37.

I3 Kang Y, Assuncao BL, Denduluri S, et al. Symptomatic Heart Failure in Acute Leukemia Patients Treated With Anthracyclines. J Am Coll Cardiol CardioOnc 2019;1:208-I7.

I4 Ezaz G, Long JB, Gross CP, Chen J. Risk prediction model for heart failure and cardiomyopathy after adjuvant trastuzumab therapy for breast cancer. J Am Heart Assoc. 20I4;3:eooo472.I

I5 Lotrionte M, Biondi-Zoccai G, Abbate A, et al. Review and meta-analysis of incidence and clinical predictors of anthracycline cardiotoxicity. Am J Cardiol. 2013;II2:1980-1984.

I6 Lefrak EA, Pitha J, Rosenheim S, Gotilieb JA. A clinicopathologic analysis of adriamycin cardiotoxicity. Cancer. 1973;32:302-I4.

I7 Saini J, Rich MW, Lyss AP. Reversibility of severe left ventricular dysfunction due to doxorubicin cardiotoxicity. Report of three cases. Ann Intern Med 1987;I06:8I4-6.

I8 Cohen M, Kronzon I, Lebowitz A. Reversible doxorubicin-induced congestive heart failure. Arch Intern Med. 1982;I42:1570 -I.

I9 Haq MM, Legha SS, Choksi J, et al. Doxorubicin-induced congestive heart failure in adults. Cancer I985;56:136I-5.

20 Jensen BV, Skovsgaard T, Nielsen SL. Functional monitoring of anthracycline cardiotoxicity: a prospective, blinded, long-term observational study of outcome in 120 patients. Ann Oncol 2002;13:699-709.

2I Tallaj JA, Franco V, Rayburn BK, et al. Response of Doxorubicin-induced Cardiomyopathy to the Current Management Strategy of Heart Failure. J Heart Lung Transplant 2005;24:2196-2OI.

22 Curigliano G, Lenihan D, Fradley M, et al. Management of cardiac disease in cancer patients throughout oncological treatment: ESMO consensus recommendations. Ann Oncol. 2020;3I(2):17II90.

23 Cardinale D, Ciceri F, Latini R, et al. Anthracycline-induced Cardiotoxicity: A Multicenter Randomised Trial Comparing Two Strategies for Guiding Prevention With Enalapril: The International CardioOncology Society-one Trial. Eur J Cancer. 2018;94:I26-137. 
24 Vaduganathan M, Hirji SA, Qamar A, et al. Efficacy of Neurohormonal Therapies in Preventing Cardiotoxicity in Patients With Cancer Undergoing Chemotherapy. J Am Coll Cardiol CardioOnc. 20I9; I(I):54-65.

25 Speyer JL, Green MD, Zeleniuch-Jacquotte A, et al. ICRF-I87 permits longer treatment with doxorubicin in women with breast cancer. Journal of Clinical Oncology I992;IO:II7-27.

26 van Dalen EC, Caron HN, Dickinson HO, Kremer LC. Cardioprotective interventions for cancer patients receiving anthracyclines. Cochrane Database Syst Rev 20II; 6: CDoo39I7.

27 FDA dexrazoxane https://www.accessdata.fda.gov/drugsatfda_docs/label/20I2/0202I2soI3lbl.pdf

28 Howlader N, Noone AM, Krapcho M, Miller D, Brest A, Yu M, Ruhl J, Tatalovich Z, Mariotto A, Lewis DR, Chen HS, Feuer EJ, Cronin KA (eds). SEER Cancer Statistics Review, I975-20I7, National Cancer Institute. Bethesda, MD, https://seer.cancer.gov/csr/I975_20I7/, based on November 20I9 SEER data submission, posted to the SEER web site, April 2020.

29 Peng J, Rushton M, Johnson C, et al. An international survey of healthcare providers' knowledge of cardiac complications of cancer treatments. Cardiooncology. 2019;5:I2. doi: I0.II86/s40959-0I9-0049-2

30 Pituskin E, Haykowsky M, McNeely M, Mackey J, Chua N, Paterson I. Rationale and design of the multidisciplinary Team IntervenTion in cArdio-oNcology study (TITAN). BMC Cancer. 20I6;16:733. 


\section{Appendices}

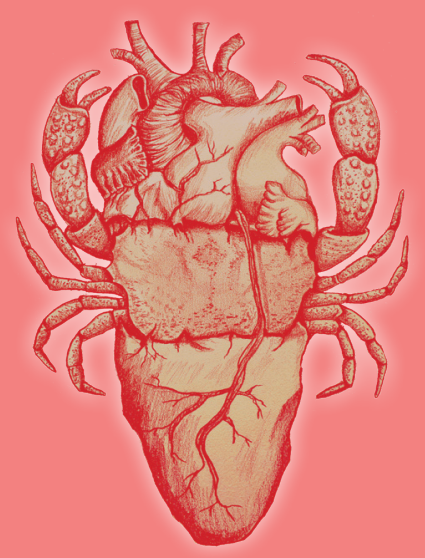


Nederlandse samenvatting

Dankwoord

Curriculum Vitae 


\section{Fundamenten voor het verbeteren van cardio-oncologische zorg}

Cardio-oncologie is een relatief nieuw subspecialisme binnen de geneeskunde, welke zich richt op de cardiovasculaire gezondheid van kankerpatiënten vooraf-, gedurende- en na het ziekteproces. Zo kan bijvoorbeeld de cardiovasculaire status van patiënten die reeds bekend zijn met cardiovasculaire ziekte geoptimaliseerd worden, om zo de meest effectieve oncologische behandeling te kunnen geven. Gedurende en na het ziekteproces kan cardiale screening bijdragen aan het detecteren van cardiovasculaire bijwerkingen van de oncologische behandeling. Bijwerkingen die kunnen optreden zijn divers, en omvatten onder andere ritmestoornissen, pericardiale ziekte, coronarialijden, klepafwijkingen en cardiale dysfunctie.

In de afgelopen decennia is de aandacht voor kankertherapie-geïnduceerde cardiovasculaire bijwerkingen sterk toegenomen. Deze aandacht was initieel voornamelijk gericht op de behandeling van cardiovasculaire bijwerkingen, en in de afgelopen jaren is de focus uitgebreid naar vroegtijdige herkenning en preventie van deze bijwerkingen. Wereldwijd zijn gespecialiseerde cardio-oncologie poliklinieken opgericht, welke een gestructureerde benadering bieden voor het opsporen- en behandelen van cardiovasculaire bijwerkingen. De cardio-oncologie poliklinieken hanteren verschillende werkwijzen, aangezien tot op heden geen(internationale)richtlijnbeschikbaarisvoor hetuitvoerenvancardio-oncologischezorg.

Het doel van de thesis, zoals beschreven in Hoofdstuk I, is het beschrijven van de implementatie- en uitkomsten van een gespecialiseerde cardio-oncologie polikliniek. Daarnaast worden ook enkele aspecten van de huidige kennis omtrent het uitvoeren van cardio-oncologische zorg geëvalueerd.

In 2015 is in het Universitair Medisch Centrum Utrecht een gespecialiseerde cardio-oncologie polikliniek opgericht. Het zorgpad wat gehanteerd wordt op deze polikliniek is beschreven in Hoofdstuk 2. Een van de kerntaken van de cardio-oncologie polikliniek is de vroegtijdige herkenning van kankertherapie-geïnduceerde cardiale dysfunctie. Deze bijwerking kan, indien deze pas wordt opgemerkt als er cardiale klachten zijn, moeilijk behandelbaar zijn en kent dan ook een hoge mortaliteit. Aangenomen wordt dat vroegtijdige herkenning en -behandeling van deze bijwerking betere uitkomsten geeft op het herstel van de cardiale functie. Zodoende wordt binnen de cardio-oncologie polikliniek screening verricht bij patiënten die een verhoogd risico lopen op cardiale dysfunctie. Binnen het zorgpad wordt gebruik gemaakt van een risicomodel om de kans op cardiale dysfunctie in te schatten (Figuur I). Dit risicomodel bevat zowel behandeling gerelateerde- als patiënt gerelateerde factoren. Oncologische middelen die een verhoogd risico op cardiale dysfunctie geven zijn onder andere anthracyclines en trastuzumab. Anthracyclines worden voornamelijk gebruikt voor de behandeling van borstkanker, hematologische maligniteiten (o.a. acute leukemie, lymfomen) en verscheidene soorten kinderkanker. Trastuzumab is een middel wat voornamelijk gebruikt wordt voor de behandeling van HER2 positieve borstkanker. 
De patiënt-gerelateerde factoren in het risicomodel zijn onder andere leeftijd, geslacht, en reeds aanwezige cardiovasculaire ziekte of -risicofactoren (o.a. hypertensie, diabetes). De risicoscore wordt gebruikt om te beslissen of patiënten in aanmerking komen voor verwijzing naar de cardio-oncologie polikliniek, en daarnaast ook om de intensiteit van follow-up te bepalen. In Hoofdstuk 2 wordt besproken welke detectiemethoden gebruikt kunnen worden en welke interventies verricht kunnen worden in het geval cardiotoxiciteit optreedt.

\section{Cardiotoxiciteit Risico Score (CRS)}

\begin{tabular}{|c|c|}
\hline Risicoscore systemische behandeling & \multicolumn{1}{c|}{ Voorbeelden } \\
\hline Hoog (score 4$)$ & $\begin{array}{l}\text { Anthracyclines }\left(>240 \mathrm{mg} / \mathrm{m}^{2}\right) \\
\text { Cyclofosfamide }\left(>1.5 \mathrm{~g} / \mathrm{m}^{2}\right)\end{array}$ \\
\hline Intermediair (score 2$)$ & $\begin{array}{l}\text { Anthracyclines }\left(\leq 240 \mathrm{mg} / \mathrm{m}^{2}\right) \\
\text { Vinblastine; Ponatinib }\end{array}$ \\
\hline Laag (score 1) & Bevacizumab; Imatinib \\
\hline Verwaarloosbaar (score 0) & Fludarabine; Rituximab \\
\hline
\end{tabular}

\begin{tabular}{|c|c|}
\hline \multicolumn{2}{|c|}{ Patientgerelateerde risicofactoren (1 punt per item) } \\
\hline Thoracale bestraling & Eerdere behandeling met anthracyclines \\
\hline Hypertensie & Cardiomyopathie / Hartfalen \\
\hline Diabetes Mellitus & Leeftijd $<15$ jaar of $>65$ jaar \\
\hline Vrouwelijk geslacht & Coronair- of perifeer arterieel vaatlijden \\
\hline
\end{tabular}

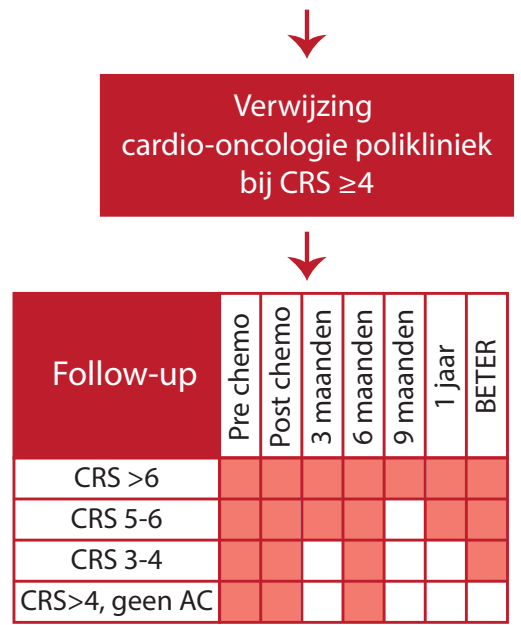

Figuur I Risicostratificatie en polikliniek cardio-oncologie. Bij een risicoscore van 4 over hoger wordt geadviseerd patiënt te verwijzen naar de polikliniek cardio-oncologie. $\mathrm{AC}=$ anthracyclines.

Om het risico op cardiotoxiciteit goed te kunnen inschatten is het noodzakelijk om te weten in hoeverre de oncologische behandeling cardiotoxisch is. Naast anthracyclines en trastuzumab wordt van verscheidene andere middelen gezegd dat zij een hoog risico geven op het ontwikkelen van cardiale dysfunctie. Middelen worden als hoog risico beschouwd indien zij in meer dan IO\% van de gevallen leiden tot cardiotoxiciteit. In Hoofdstuk 3 wordt 
het cardiotoxische profiel van 5 'hoog-risico' chemotherapeutica geëvalueerd, namelijk mitomycine C, ifosfamide, vincristine, cyclofosfamide en clofarabine. Een belangrijke bevinding van deze studie is dat de hoge incidentie van cardiotoxiciteit gebaseerd is op studies waarin patiënten, naast de geëvalueerde middelen, ook behandeling ondergingen met andere cardiotoxische middelen zoals anthracyclines. Hierdoor is onvoldoende duidelijk of de middelen van zichzelf daadwerkelijk cardiotoxisch zijn. De bevindingen uit Hoofdstuk 3 laten zien dat de huidige kennis omtrent de cardiotoxiciteit van deze middelen beperkt is. Met deze beperkingen dient rekening gehouden te worden indien de incidentie cijfers gebruikt worden voor risicostratificatie. Daarnaast wordt aanbevolen om studies te verrichten naar de cardiovasculaire bijwerkingen in patienten die middelen als monotherapie ontvangen. Aangezien veel middelen nog beperkt als monotherapie gegeven worden, zou het ook interessant zijn om de cardiovasculaire toxiciteit van veelgebruikte chemokuren te analyseren.

De aanbevelingen uit Hoofdstuk 3 zijn geïmplementeerd in Hoofdstuk 4. In deze studie is op een systematische manier het cardiotoxiciteitsprofiel van de chemokuur R-CHOP onderzocht. Deze chemokuur bevat de middelen rituximab, cyclofosfamide, doxorubicine, vincristine en prednisolon en wordt sinds 1976 gebruikt voor de behandeling van agressieve non-Hodgkin lymfomen. Van twee middelen uit deze kuur, namelijk cyclofosfamide en de anthracycline doxorubicine, is bekend dat zij een verhoogd risico op cardiale dysfunctie geven. Voor de systematische analyse zijn uitkomsten van I37 studies (2I.2III patiënten) samengevoegd. Ernstige cardiovasculaire bijwerkingen werden gezien in $2.4 \%$ van de patiënten en cardiale dysfunctie werd in $4.6 \%$ van de patiënten gevonden. Opmerkelijk was dat, indien cardiale screening na behandeling verricht werd, in $\mathrm{II.7 \%}$ van de patiënten cardiale dysfunctie gevonden werd. Dit percentage was significant hoger dan het percentage patiënten waarbij geen cardiale screening verricht werd na behandeling (I.6\%). Dit impliceert dat, indien geen cardiale screening verricht wordt, de bijwerking van cardiale dysfunctie vaak onopgemerkt blijft. Concluderend lijkt cardiale dysfunctie een veelvoorkomende bijwerking te zijn in patiënten die behandeld worden met R-CHOP. Cardiale screening kan bijdragen om deze bijwerking vroeg te detecteren en zo nodig te behandelen om verdere achteruitgang van de hartfunctie te voorkomen.

Hoofdstuk 5 beschrijft de echocardiografische kenmerken en de respons op hartfalentherapie van 92 patiënten met anthracycline-geïnduceerde cardiale dysfunctie die op de cardio-oncologie polikliniek gezien zijn. In deze observationele studie is onder andere gekeken of er verschillen zijn tussen patiënten die vroeg- en laat na anthracycline-bevattende therapie gediagnosticeerd zijn met cardiale dysfunctie. Het merendeel van de patiënten $(83 \%)$ had milde cardiale dysfunctie, en $94 \%$ van de patiënten had geen- of milde cardiale klachten. Echocardiografisch werden geen verschillen gevonden tussen patiënten waarbij vroeg- en laat na anthracycline therapie cardiale dysfunctie werd vastgesteld. Wel werd in patiënten die vroeg gediagnosticeerd waren en waarbij hartfalentherapie gestart was, een beter herstel van de cardiale functie gezien, ten opzichte van patiënten die laat na anthracycline-bevattende therapie gediagnosticeerd waren. Dit impliceert dat vroege herkenning 
van anthracycline-geïnduceerde cardiale dysfunctie belangrijk is voor een goede respons van hartfalentherapie.

In Hoofdstuk 6 worden uitkomsten geëvalueerd van de verrichte cardio-oncologische zorg van April 2015 tot en met September 20I9. In deze periode is aan 800 patiënten zorg geleverd volgens het cardio-oncologische zorgpad zoals beschreven in Hoofdstuk 2. De meest voorkomende kankersoorten waren borstkanker ( $\mathrm{n}=192 ; 24 \%)$, lymfomen $(\mathrm{n}=\mathrm{I} 78 ; 22 \%)$ en acute leukemie $(\mathrm{n}=\mathrm{I} 74 ; 22 \%)$. Patiënten werden voornamelijk naar de cardio-oncologie polikliniek verwezen voor cardiale screening vanwege een verhoogd risico op cardiotoxiciteit. $\mathrm{Bij} 497$ patiënten (62\%) werd dit tijdens therapie gedaan, en I42 patiënten (I8\%) werden gescreend nadat zij in het verleden behandeld werden met potentieel cardiotoxische kankerbehandeling. Cardiale klachten waren de reden voor verwijzing in de overige I6I patiënten (20\%). Cardiovasculaire bijwerkingen die werden gediagnosticeerd waren onder andere cardiale dysfunctie, hartritmestoornissen, pericardiale ziekte, coronarialijden, hartkleplijden en pulmonale hypertensie. Tijdens een mediane follow-up duur van I4.3 maanden werd in 36\% van de patiënten cardiovasculaire bijwerkingen gediagnosticeerd. Cardiale dysfunctie was de meest voorkomende cardiale bijwerking ( $\mathrm{n}=\mathrm{I} 76 ; 22 \%)$. In het merendeel (84\%) van de gevallen kon, ondanks de cardiovasculaire bijwerkingen, de oncologische behandeling wel worden afgemaakt.

Om de lange-termijn effecten van cardio-oncologische zorg op zowel oncologische- en cardiale uitkomsten te evalueren is de ONCOR registratie opgericht. Deze nationale studie verzamelt op een systematische manier gegevens van cardio-oncologische zorg. De studie wordt uitgevoerd in ziekenhuizen waarin het cardio-oncologie zorgpad, zoals beschreven is in Hoofdstuk 2, is geïmplementeerd. In Hoofdstuk 7 is de studie-opzet van de ONCOR registratie beschreven, met daarbij uitkomsten van de reeds verzamelde data van II42 patienten. Het uiteindelijke doel van de ONCOR registratie is om de cardio-oncologische zorg te optimaliseren om zo de cardiovasculaire gezondheid van kankerpatiënten te verbeteren.

In Hoofdstuk 8 worden de bevindingen uit deze thesis bediscussieerd en worden suggesties genoemd voor verder onderzoek. Er wordt geconcludeerd dat het subspecialisme 'cardio-oncologie' zich sterk heeft ontwikkeld in de afgelopen jaren, maar dat er nog verscheidene kennishiaten zijn ten aanzien van het verrichten van de optimale cardio-oncologische zorg. Om de klinische cardio-oncologische zorg te verbeteren wordt aanbevolen om de kennis omtrent cardiotoxische profielen van oncologische middelen en -chemokuren te verbeteren. Daarnaast is er behoefte aan een gevalideerde risicoscore op basis waarvan patiënten geselecteerd kunnen worden die baat hebben bij specialistische cardio-oncologische zorg. Tenslotte is het noodzakelijk om lange-termijn uitkomsten van patiënten die cardio-oncologische zorg hebben ontvangen te evalueren, om zo een compleet beeld te krijgen van de invloed van specialistische zorg op zowel cardiale- als oncologische uitkomsten. 


\section{Dankwoord}

Graag wil ik iedereen bedanken die mij heeft gesteund in de afgelopen jaren en heeft bijgedragen aan het tot stand komen van dit proefschrift. De volgende mensen wil ik in het bijzonder bedanken.

Geachte professor Doevendans, beste Pieter, bedankt voor de begeleiding en ondersteuning tijdens mijn promotietraject. De gesprekken met u zorgden ervoor dat ik weer met een frisse blik naar mijn onderzoek keek.

Geachte professor Asselbergs, beste Folkert, bedankt voor al je hulp de afgelopen jaren. Ondanks dat ik niet altijd bij de Asselbergs meetings kon zijn, was je toch goed op de hoogte van mijn vorderingen en bood je altijd je hulp aan. Als ik vragen had kon ik altijd rekenen op een snelle reactie, dank daarvoor!

Dr. Cramer, beste Maarten Jan, bedankt voor je enthousiasme om van mijn promotietijd 'de mooiste tijd van je leven' te maken! De avonden bij het benefiet golftoernooi in 't Gooi zullen me altijd bijblijven.

Dr. Teske, beste Arco. Als echocardiografie expert heb je me veel bij kunnen brengen. Daarnaast ben ik je dankbaar voor de mogelijkheid om onder jouw begeleiding klinische werkzaamheden uit te voeren op de polikliniek cardio-oncologie.

Graag wil ik alle patienten bedanken die hebben willen deelnemen aan de onderzoeken. Ook wil ik de medewerkers van het Alexander Monro Ziekenhuis, en in het bijzonder Emine Goker en Thonny van Beest, bedanken voor hun hulp bij het opzetten van de EFACT studie.

De leden van de beoordelingscommissie, te weten prof. dr. ir. H.M. den Ruijter, prof. dr. T. Leiner, prof. dr. E. Van der Wall, prof. dr. L.C.M. Kremer, prof. dr. H.M. Verkooijen, wil ik bedanken voor het beoordelen van mijn proefschrift.

Alle co-auteurs wil ik bedanken voor de samenwerking en hun bijdrage aan dit proefschrift.

Mijn collega promovendi wil ik bedanken voor alle gezelligheid binnen en buiten het UMCU. De Asselbergs onderzoekers in het bijzonder, jullie gaven het kantoorleven de afgelopen 3 jaar een stuk meer glans, dank! 
Mimount en Marijke, bedankt dat jullie naast mij willen staan tijdens mijn promotie.

Mimount, dank voor je luisterend oor, goede adviezen en bovenal alle gezelligheid.

Marijke, cardio-onco buddy! Bedankt voor het sparren, de congresbezoekjes en de fijne samenwerking. Ik hoop dat we onze dagstarts, dagevaluaties en etentjes nog lang zullen voortzetten.

Ten slotte Patrick, mijn ouders, Michelle en Bram. Michelle, ik ben trots op je creatieve talent en ben blij dat ik er gebruik van mocht maken bij het ontwerpen van de kaft. Pap en mam, bedankt voor jullie steun en dat ik altijd bij jullie terecht kan. Lieve Patrick, bedankt voor al je hulp op de achtergrond, zeker tijdens de laatste loodjes van mijn promotie onderzoek. Dank voor alles! 


\section{Curriculum Vitae}

Janine Anne Maria Kamphuis was born on the $3^{\text {rd }}$ of August, 1990 in Oldenzaal, the Netherlands. She graduaded from the Gymnasium of the Twents Carmel College, Oldenzaal in 2008. In 2008 she moved to Groningen to study Human Movement Sciences, from which she obtained her Bachelor degree in 2012. In 2009 she started with Medical school at the Rijksuniversiteit Groningen, from which she obtained her Master of Science in Medicine in 2015. She did her senior internships at the department of Cardiology at Medisch Spectrum Twente Enschede and the department of Sports Medicine at Isala Zwolle.

After graduating from Medical school, she started working as a cardiology resident (ANIOS) at Deventer Ziekenhuis. In 2017 she started as a $\mathrm{PhD}$ student at the University Medical Center Utrecht, supervised by prof. dr. P.A.F.M. Doevendans, prof. dr. F.W. Asselbergs, dr. M.J.M. Cramer and dr. A.J. Teske, which resulted in this thesis. In May 2020 she has started working as a resident in Cardiology (ANIOS) at Medisch Spectrum Twente. In 202I she will start with her specialist training in Cardiology under supervision of dr. P.M.J. Verhorst and dr. L.J. Wagenaar.

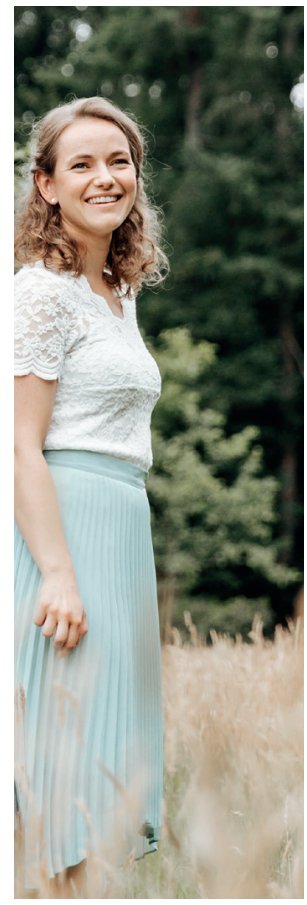


